\title{
K-THEORY FOR TRIANGULATED CATEGORIES II: THE SUBTLETY OF THE THEORY AND POTENTIAL PITFALLS*
}

\author{
AMNON NEEMAN ${ }^{\dagger}$
}

0. Introduction. This is the third instalment of a series, and it is perhaps best to briefly review the contents of the others. Given a triangulated category $\mathcal{T}$, it is possible to attach to it a $K$-theory space. This space has a delooping. Despite the best efforts of several friends, the author perversely insists on calling this delooping

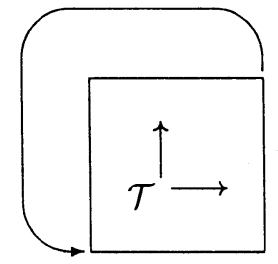

. The key theorem of this series of articles is that this definition

passes some test of being sensible. We remind the reader:

Strong Theorem I.7.1. Let $\mathcal{T}$ be a small triangulated category with a t-structure. Assume $\mathcal{T}$ has at least one model. Let $\mathcal{A}$ be the heart of the $t$-structure. Let $\mathcal{T}^{b}$ be the bounded part of $\mathcal{T}$. In particular, if the t-structure is non-degenerate, $\mathcal{T}^{b}=\mathcal{T}$. With the simplicial set

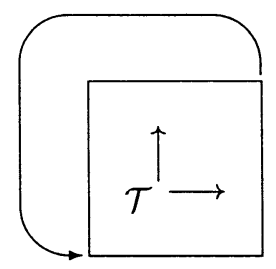

defined appropriately, the natural map
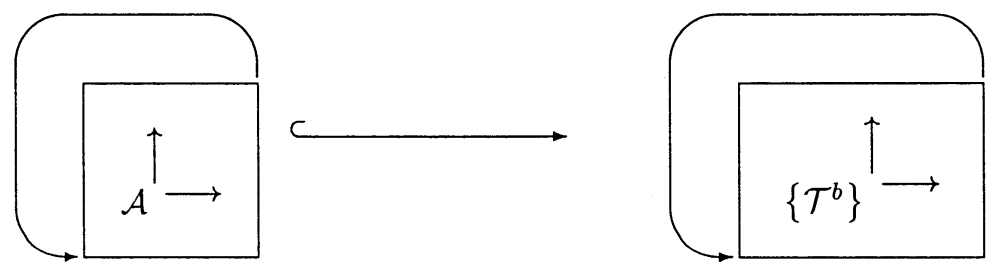

induces a homotopy equivalence.

The reader will remember that the problem of appropriately defining the simplicial set

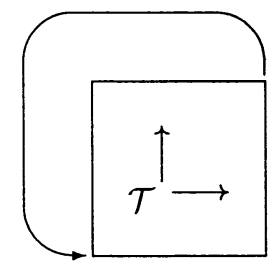

${ }^{*}$ Received April 30, 1997; accepted for publication September 20, 1997.

tDepartment of Mathematics, University of Virginia, Charlottesville, Virginia 22903 USA (an3r@virginia.edu). 
is delicate; the introduction to $K$-theory for triangulated categories $I$ offers a more detailed discussion of this point. In any case, for the bounded derived category of an abelian category $\mathcal{A}$, this allows us to recover, from the derived category $D^{b}(\mathcal{A})$, Quillen's usual $K$-theory of $\mathcal{A}$.

In this article we will analyse why the proof must, of its nature, be somewhat difficult. Precisely, by slightly modifying the statements, one gets patently false results. And it is interesting to notice just how much of the proof goes through in this altered framework. The real purpose of this article is to warn the unwary beginner of the pitfalls in the theory. The article is only really of interest to the expert seeking to improve the results. There is much room for improvement, so it is to be hoped that someone will work on it.

This article is most certainly not independent of the others in the series. In the introduction to $K$-theory for triangulated categories $I$, I divided up the readers of any piece of mathematics into three broad groups, listed in order of probable size:

Group 1: The people who want a rough idea of the contents of the article, and at the very most a sketch of the proofs in an easy special case.

Group 2: The people who want to check the result, because they might consider using it in their own work.

Group 3: The people reading the article because they might work on the problem themselves.

The first two parts of this series, $K$-theory for triangulated categories $I$, were intended for a Group 1 audience. The parts that will follow, $K$-theory for triangulated categories III, are primarily for Group 2. The present part is mostly for the benefit of Group 3. Don't say you have not been warned.

In $K$-theory for triangulated categories $I$, we introduce the definitions and notation (this takes us more than 80 pages), and then we give the simplest proof of the simplest version of our theorem: the $K$-theory of an abelian category is a retract of the $K-$ theory of its derived category. All the readers of subsequent articles are assumed to be familiar with the notation. So you should have read at least the first 80 pages of $K$-theory for triangulated categories $I$ if you read beyond this word. In fact, it is highly advisable to have skimmed through the rest of $K$-theory for triangulated categories I. There is a little more notation introduced in the last two sections, but even more relevant is that there is a relatively gentle introduction to the way the proofs work, and the type of simplicial sets one constructs.

The first section of $K$-theory for triangulated categories III, Section III.1, is also recommended reading before one begins with the current article. There are two types of homotopy that I know, for the simplicial sets that come up in triangulated $K$ theory. The first type is the trivial homotopies. These are the triangulated analogues of contractions to an initial or a terminal object. The second type of homotopy is the non-trivial homotopies. And one of the key features of this theory is that there is really only one of the non-trivial homotopies.

The first problem one should address is why the various homotopies that come up in the proof are well defined. In other words, in $K$-theory for triangulated categories $I$ the author tried to convince the reader that there are very few homotopies in this theory. The approach of the proof is to apply the few, overworked homotopies that there are, to a wide assortment of simplicial sets. If one is ingenious enough about it, one ends up with a proof of an interesting theorem.

In Sections 1 and III.1, the author shows how this can be made very precise. 
Aside from the "trivial" homotopies of contraction and truncation, there is only one non-trivial homotopy. Precisely, all the non-trivial homotopies we have seen can be deduced from a blueprint homotopy, by deletion and subdivision.

Section III.1, being written for a Group 2 audience, takes the optimistic view and focuses on the one "non-trivial" homotopy. The reason is that it turns out to be easy to give a very satisfactory account of it, and explain in some generality why it always does what it should.

Section 1, which is intended for you, the Group 3 reader, takes a more pessimistic view. It focuses instead on the one "trivial" homotopy that requires care. This is the $t$-structure truncation. Although seemingly very innocuous, it is treacherous because it frequently depends on a choice of differentials.

Both Section 1 and Section III.1 introduce a little notation, although most of the notation has already been encountered in $K$-theory for triangulated categories $I$. There is a great deal of overlap in the material the two sections treat. Before going on to Section 2, it would be best if the reader read at least one, preferably both of Sections 1 and III.1. These sections are quite soft. Of the two, Section 1 has more philosophy and motivation, and is peppered with examples of where prototype arguments can go wrong.

So far, I have told the reader that Sections 1 and III.1 offer a technical discussion of the homotopies that come up in the argument, and whether they are well-defined. Hardly sounds like exciting reading. Even if we are willing to concede that perhaps such a discussion should appear somewhere. What are the exciting results to be found in this article?

To describe them, we need first to review some of the material covered in $K-$ theory for triangulated categories $I$. Let $\mathcal{A}$ be an abelian category, and let $G r^{b}(\mathcal{A})$ be the category of bounded, $\mathbb{Z}$-graded objects in $\mathcal{A}$. In a way that closely parallels triangulated $K$-theory, it is possible to define simplicial sets
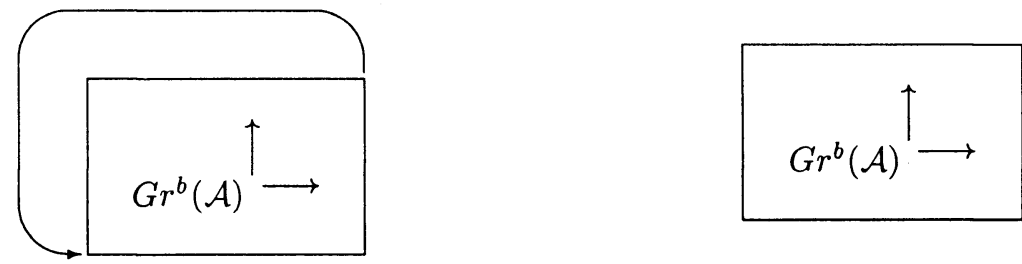

In $K$-theory for triangulated categories $I$ we proved Theorem I.4.8, which says

Theorem I.4.8. The natural inclusion

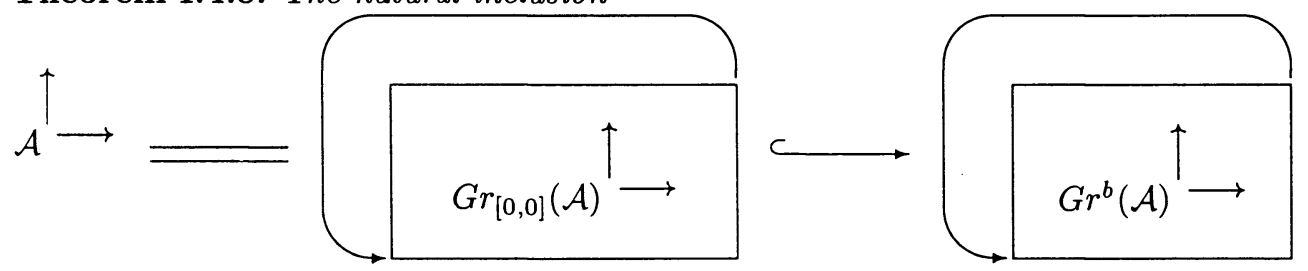

induces a homotopy equivalence.

But as we pointed out in $K$-theory for triangulated categories $I$, much of the argument works also for the simplicial set $G r^{b}(\mathcal{A}) \longrightarrow$. In Section 2 we analyse this 
very precisely. The simplicial set

$$
G r^{b}(\mathcal{A}) \longrightarrow
$$

is ridiculous. But nevertheless

we prove:

Summary of the results in Section 2. As in K-theory for triangulated categories $I$, let $G r_{[m, n]}(\mathcal{A})$ stand for the full subcategory of $G r^{b}(\mathcal{A})$ whose objects are supported on the interval $[m, n] \subset \mathbb{Z}$. In particular, $G_{[0,1]}(\mathcal{A})$ are the graded objects living in degrees 0 and 1 . We have that a delooping of the inclusion of the $K$-theory of $\mathcal{A}$ into the $K$-theory of $G r^{b}(\mathcal{A})$ factors as

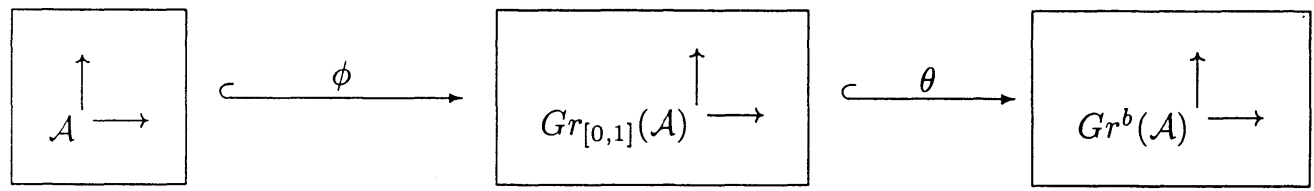

Let $F_{1}$ be the homotopy fiber of $\phi$. With this notation, we prove that

0.0.1. $\Pi_{i}(\theta)$ is injective. That is, the map $\theta$ induces an injection on homotopy groups.

0.0.2. More precisely, the group

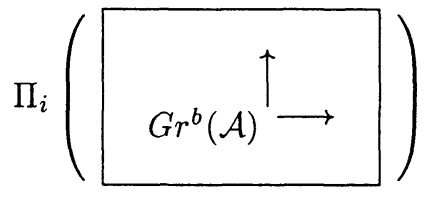

is an extension of its subgroup

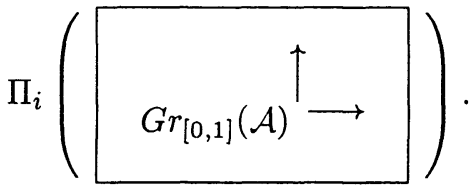

We prove that it is a countably infinite sequence of extensions by $\Pi_{i-1}\left(F_{1}\right)$. Even more precisely, for any $m<n$, the inclusions

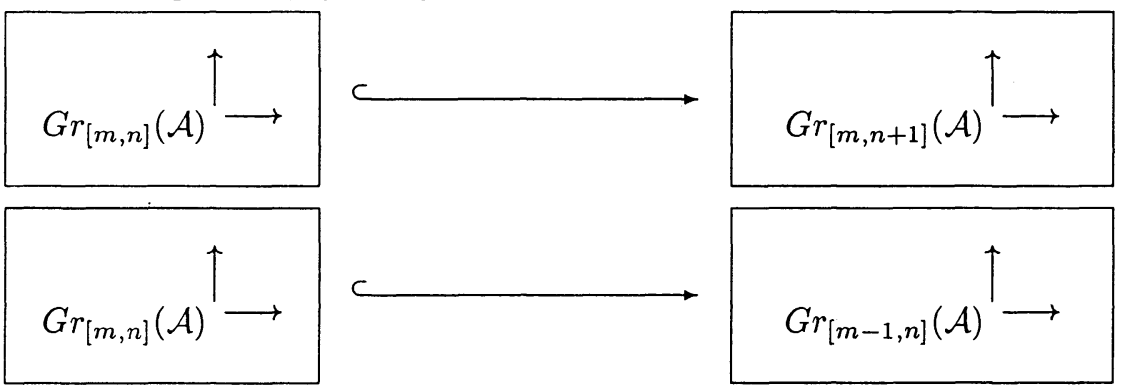

all induce injections on homotopy groups, and the groups

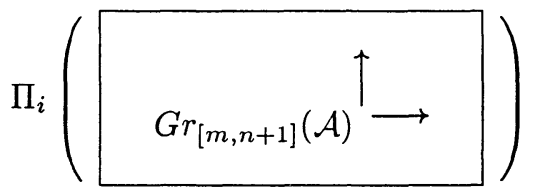

and

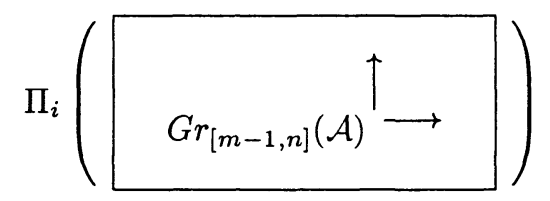


are both extensions of the subgroup $\Pi_{i}\left({ }_{[m, n]}(\mathcal{A}) \longrightarrow\right.$ This means that, if the map $\phi$ is not injective, that is there is a kernel to the map

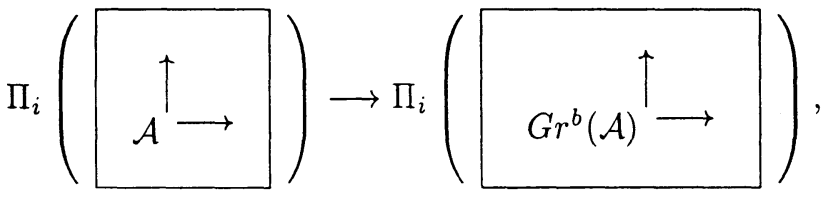

then this kernel is a quotient module of $\Pi_{i}\left(F_{1}\right)$, and hence occurs infinitely often in

$$
\Pi_{i+1}\left(\underset{G r^{b}(\mathcal{A}) \longrightarrow}{\longrightarrow}\right)
$$

Although we do not know that the $K$-theory of $\mathcal{A}$ is isomorphic to the $K$-theory of $G r^{b}(\mathcal{A})$, we know that the latter is at least as big as the former; it contains, up to passing to the associated graded of suitable (infinite) filtrations, all of the former.

We remind the reader just how absurd the definition of $G r^{b}(\mathcal{A}) \longrightarrow$ really was: see also Construction I.4.6. One begins quite reasonably. A simplex is a diagram

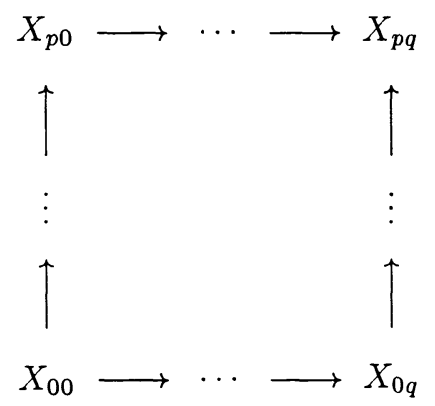

where for $0 \leq i \leq i^{\prime} \leq p, 0 \leq j \leq j^{\prime} \leq q$, the sequence

$$
X_{i j} \longrightarrow X_{i^{\prime} j} \oplus X_{i j^{\prime}} \longrightarrow X_{i^{\prime} j^{\prime}}
$$

is exact in the middle. Far less reasonable is the next condition, which says that the cokernel of the map $X_{i^{\prime} j} \oplus X_{i j^{\prime}} \longrightarrow X_{i^{\prime} j^{\prime}}$ agrees with the suspension of the kernel of $X_{i j} \longrightarrow X_{i^{\prime} j} \oplus X_{i j^{\prime}}$ only up to passing to the associated graded of some (finite) filtration. But this is the simplicial set for which we will prove, as we have just summarised, that in some sense it contains all the information encoded in Quillen's $K$-theory of $\mathcal{A}$.

This section is intended as a warning to the unwary of the many pitfalls in this theory. An innocuous change of simplicial sets can destroy the $K$-theory, leaving many 
of the arguments intact. Somewhere in the proof must be some subtle argument that fails for the perturbed simplicial set.

In $K$-theory for triangulated categories III, we prove Theorem I.7.1 in its strongest form. We recall again

Theorem I.7.1, strong version. Let $\mathcal{T}$ be a triangulated category with a nondegenerate t-structure. Assume $\mathcal{T}$ has at least one model. Let $\mathcal{A}$ be the heart. Then the inclusion

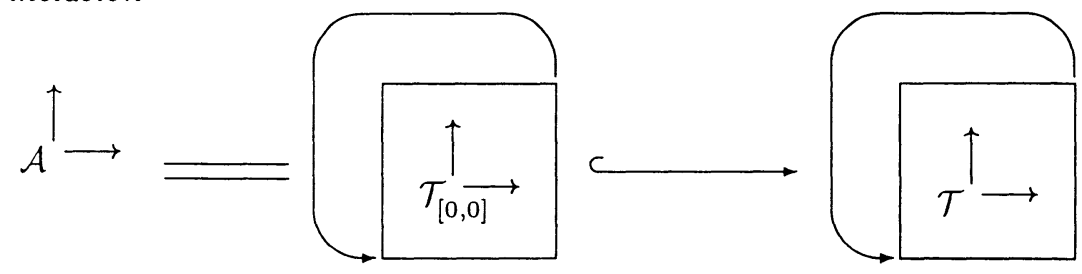

induces a homotopy equivalence.

For the special case of the derived category of an abelian category $\mathcal{A}$, with its usual $t$-structure, this reduces to:

Theorem I.7.1, weak case. Let $\mathcal{T}=D^{b}(\mathcal{A})$ be the derived category of an abelian category $\mathcal{A}$. Then the inclusion

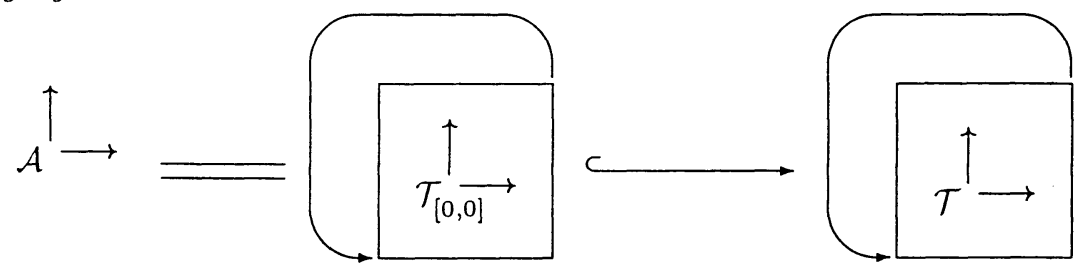

induces a homotopy equivalence.

In Section 3 we will prove the weak case. In K-theory for triangulated categories III we will prove a better theorem, namely the strong case of Theorem I.7.1. This immediately raises the question of why we bother giving the proof of the special case at all.

The reason is that the proof given here, although not much simpler than the more general argument of $K$-theory for triangulated categories III, is nevertheless closer to the simple idea that motivated both arguments. In this article, we give not only the proof, but also an indication of where it came from. We explain the simple argument the author initially had, but where the author could not fill in all the gaps in the proofs. Then we explain why the argument that actually works is just a bastardised version of the simple idea. And finally, we reason that a proof that is too close to the original simple idea is suspect, because it would fail to distinguish the construction with the differentials from the one without. I think this could be quite valuable to a Group 3 reader, although probably to no one else. One comment I would like to add is that, in the author's opinion, if there is a simpler proof of Theorem I.7.1, it will be closer to the argument of Section 3 than to anything in $K$-theory for triangulated categories III. 
1. Postmortem of the Proof of Theorem I.4.8. As the reader may have noticed by now, there are very few homotopies in this article. There are a number of trivial ones; for instance, the contraction to an initial or terminal object. Other than these, there is really only one extra, slightly non-trivial homotopy. What we will do in this section is develop a general formalism for referring to the homotopies we have already seen, and then we will go in detail over several of the arguments of Sections I.7 and I.8 to show why the homotopies there really are examples of the prototypes developed here.

The simplicial sets in the article are complicated arrays of rectangles, lines and triangles, with connecting arrows between them. For example,

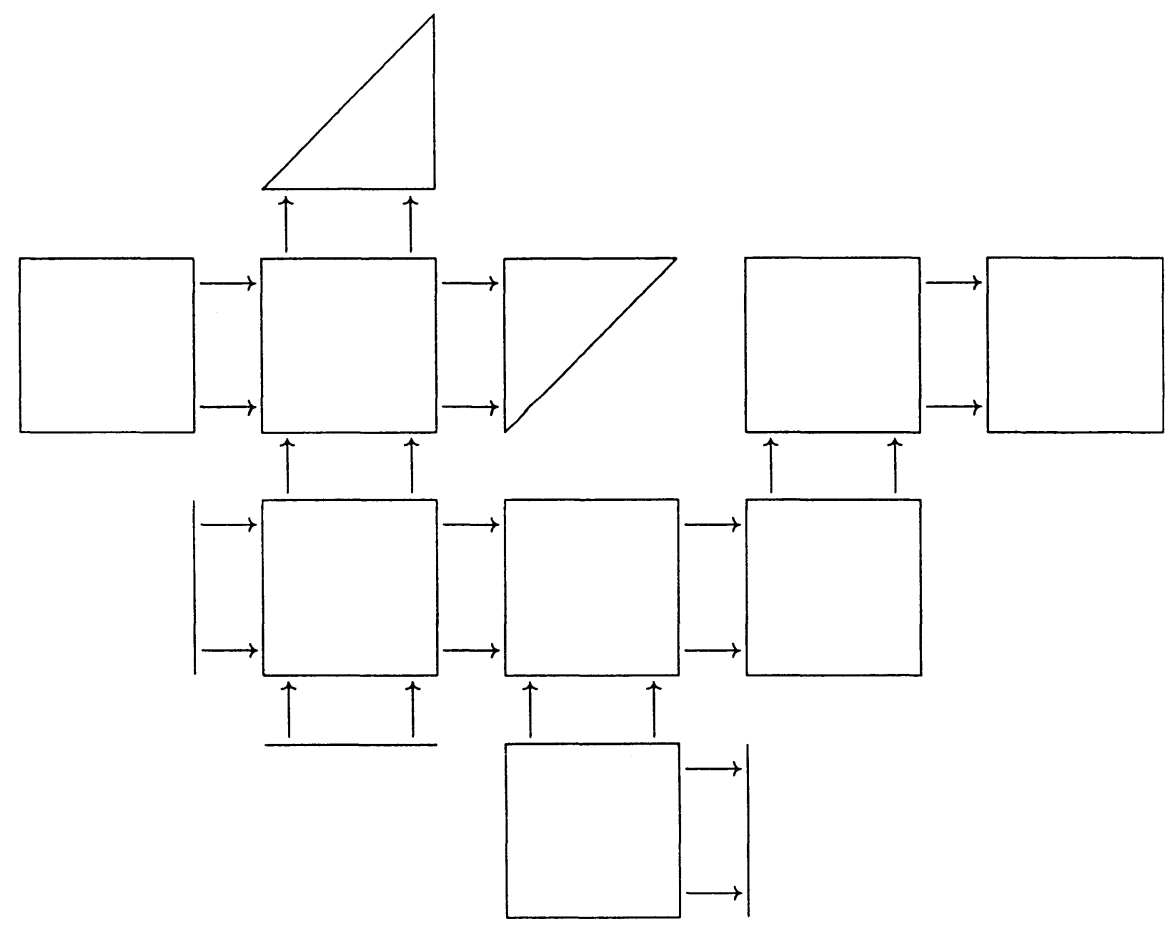

Inside the triangles we generally insert zeros. Inside the rectangles, we insert some thick subcategory of the category $\mathcal{T}$, together with a description of restrictions on the vertical and horizontal morphisms. The lines behave like very thin rectangles; they admit either a vertical or a horizontal arrow, but not both. Sometimes we are lazy, and allow ourselves to insert a zero in a rectangle; this means the restrictions on what objects and morphisms occur in the rectangle are the ones forced by its location. In this section, we want to be very general. We will write $\mathcal{T}_{*}^{?}$ ? to indicate that the thick subcategory $\mathcal{T}_{*}$ is unspecified, and the horizontal and vertical arrows are left undecided.

When the data in one of the rectangles or triangles is held fixed, it is denoted by a Roman capital letter. Thus the simplicial set 


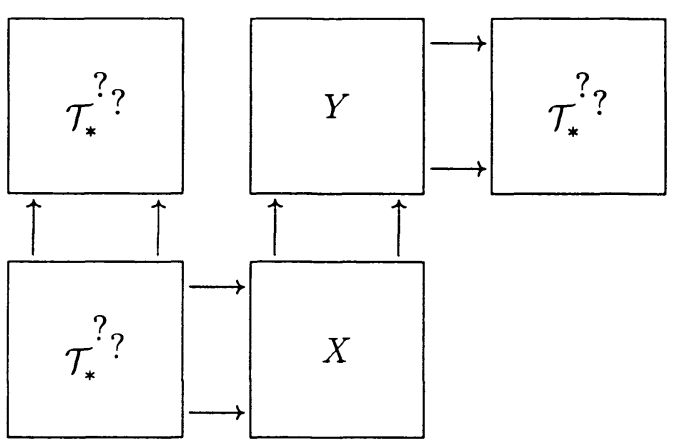

has only three simplicial structures, since the $X^{\prime} s$ and $Y^{\prime} s$ are held fixed. We have not yet seen simplicial sets as elaborate as this one; for this reason let me stop a second to write down a typical simplex. The simplex $s_{p r L}$ is given by a diagram

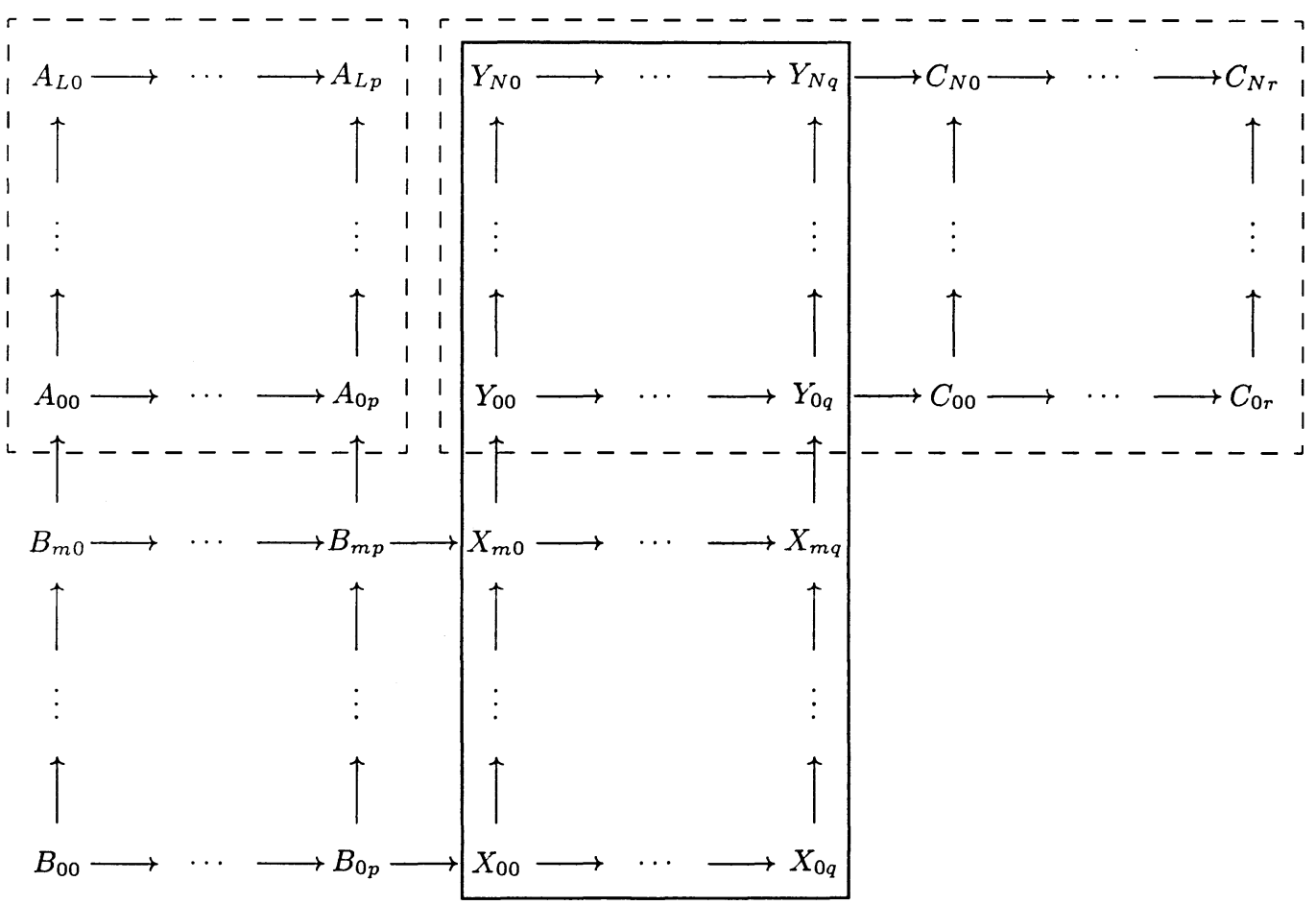

As always, the notation is that the fixed data has a thick black box around it. The reason we have highlighted the top boxes is to stress that they are unconnected. The number $L$ of rows in one is different from the number $N$ of rows in the other. There are no arrows joining the boxes. Because we fixed $X$ and $Y$, the integers $q, m$ and $N$ are fixed. The remaining three simplicial structures arise by varying $p, r$ and $L$.

In general, the arrows connecting the boxes may be restricted too. For now, we ignore the possibility.

What homotopies occur in the article? There are the trivial ones; contraction to the initial or terminal object. So in the trisimplicial set above, there is a homotopy which we denote 


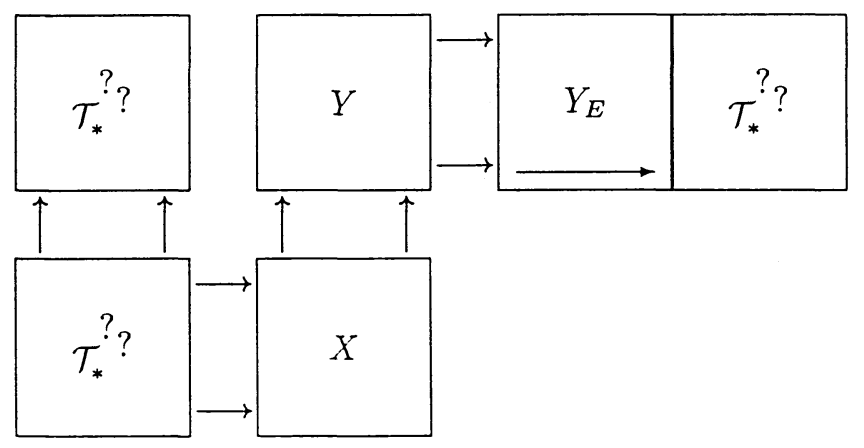

This homotopy is nothing other than the contraction to the initial object in the one rectangle that looks interesting. But unlike most of the contractions we have seen so far, here there are some other simplicial structures. Precisely, we have simplicial structures corresponding to the integers $p, L$ and $r$. Our homotopy affects only the integer $r$. Such homotopies, which will frequently occur in subsequent sections, are realised by first realising the $r$ structure to get a homotopy of the bisimplicial maps of bisimplicial spaces, and then gluing these.

This section is meant to be somewhat independent of the earlier ones, so at the risk of boring some readers let me write down a typical simplex in the homotopy

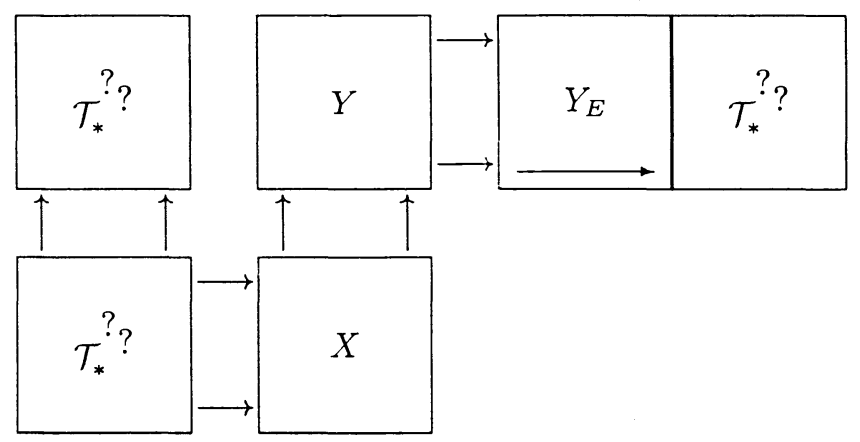

The homotopy must associate to the simplex $s_{p r L}$ an ordered set of $r+1$ simplices. The $i^{\text {th }}$ of these is the picture 


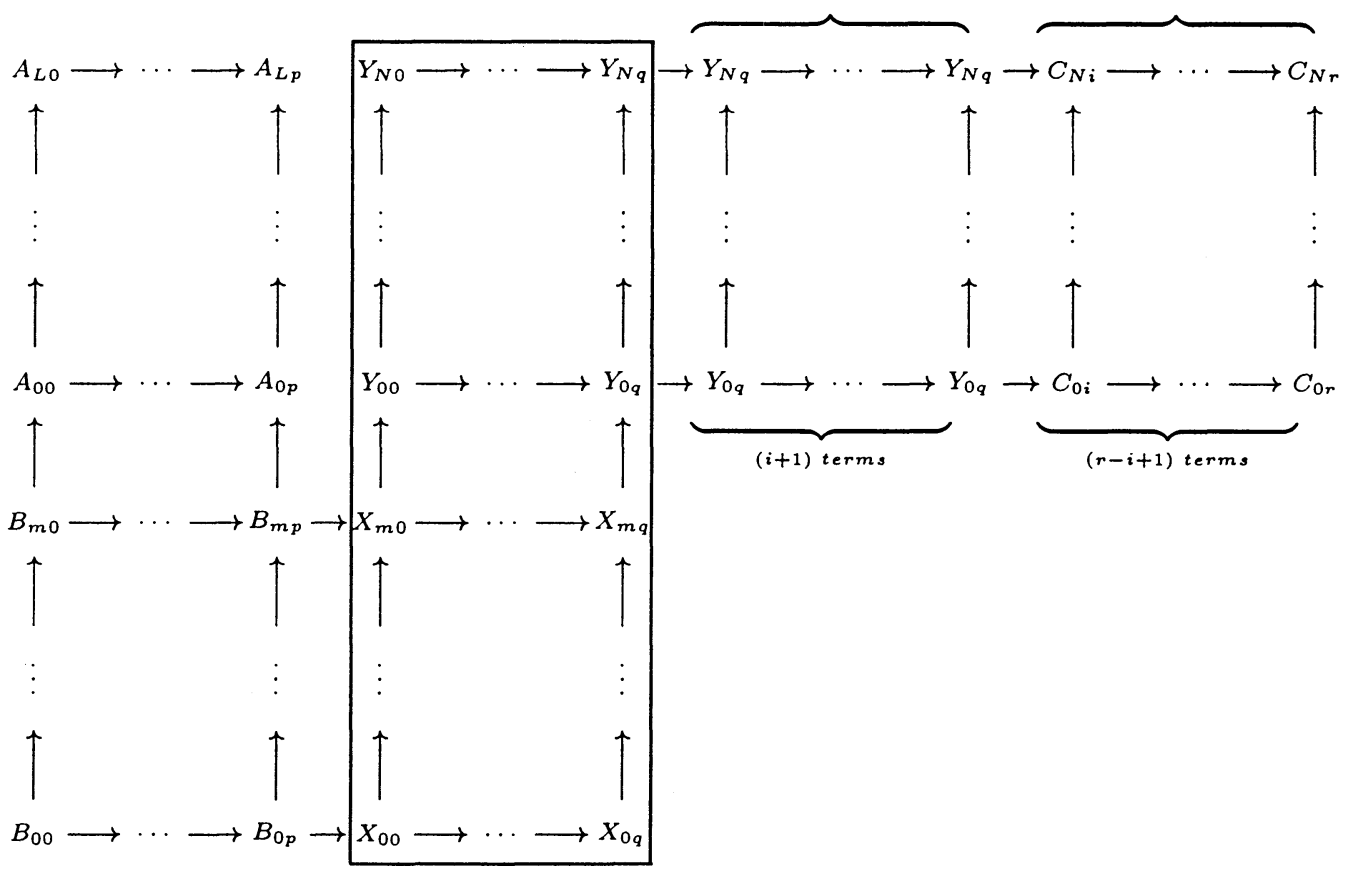

And the reason for the notation is that we want to think of the east face of $Y$, denoted $Y_{E}$, as migrating across the rectangle.

Similarly, the contraction to the initial object in

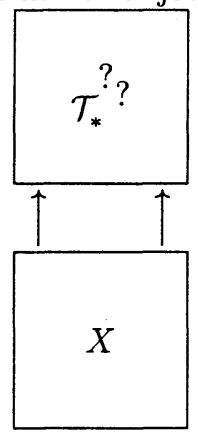

is henceforth denoted

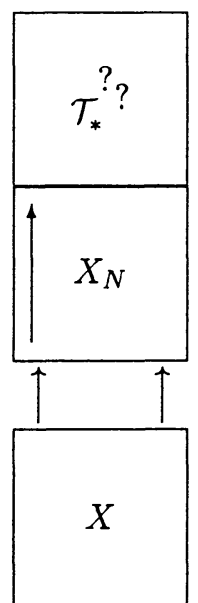


since this time the north face of $X$ is migrating. The null map which is at the end of the homotopy will be referred to by the symbol

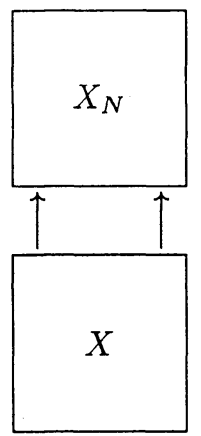

And since nothing is migrating anymore, we will leave out the arrow.

There are, of course, slight variants of this. For instance, if $X$ lies in $\mathcal{T}_{[0, n]}^{\mp}$ and $\mathcal{T}_{*}^{?} ?=\mathcal{T}_{[1, n]}^{\longrightarrow}$, then $X_{N}$ cannot be thought of as the initial object. An $n$-simplex $s_{n}$ is given by a diagram

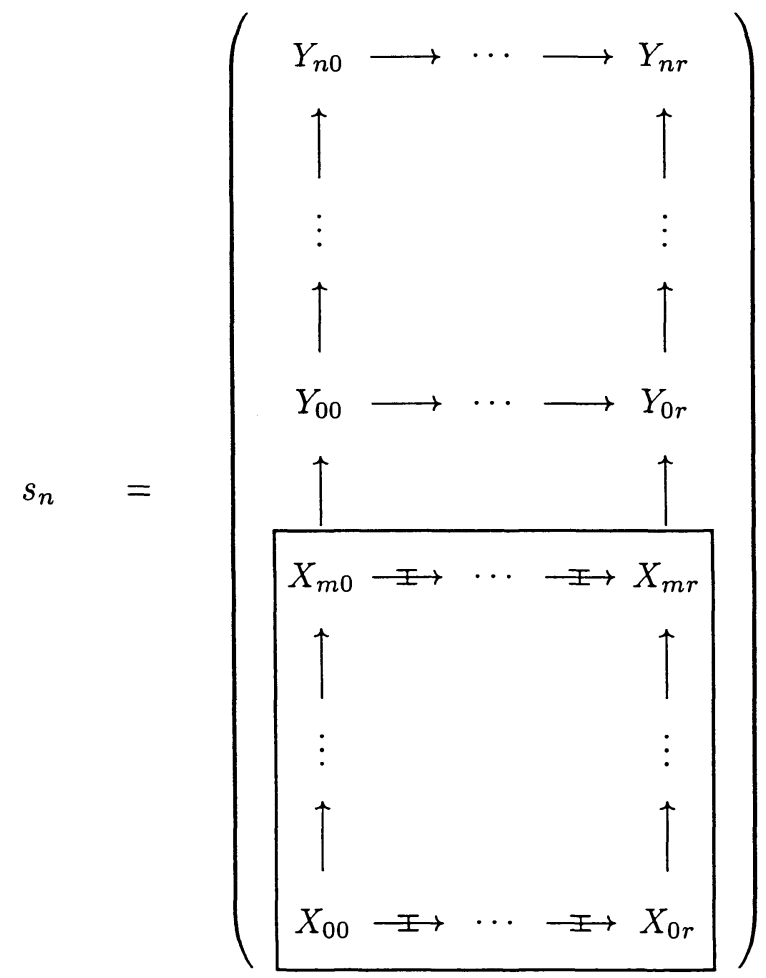

There is, of course, a candidate contraction to the "initial object." The morphisms $X_{i j} \rightarrow Y_{i^{\prime} j^{\prime}}$ all factor canonically through $X_{i j}^{>0}$ (because $Y_{i^{\prime} j^{\prime}}$ is in $\mathcal{T}^{\geq 1}$ ). Thus we can have a homotopy whose cells are 


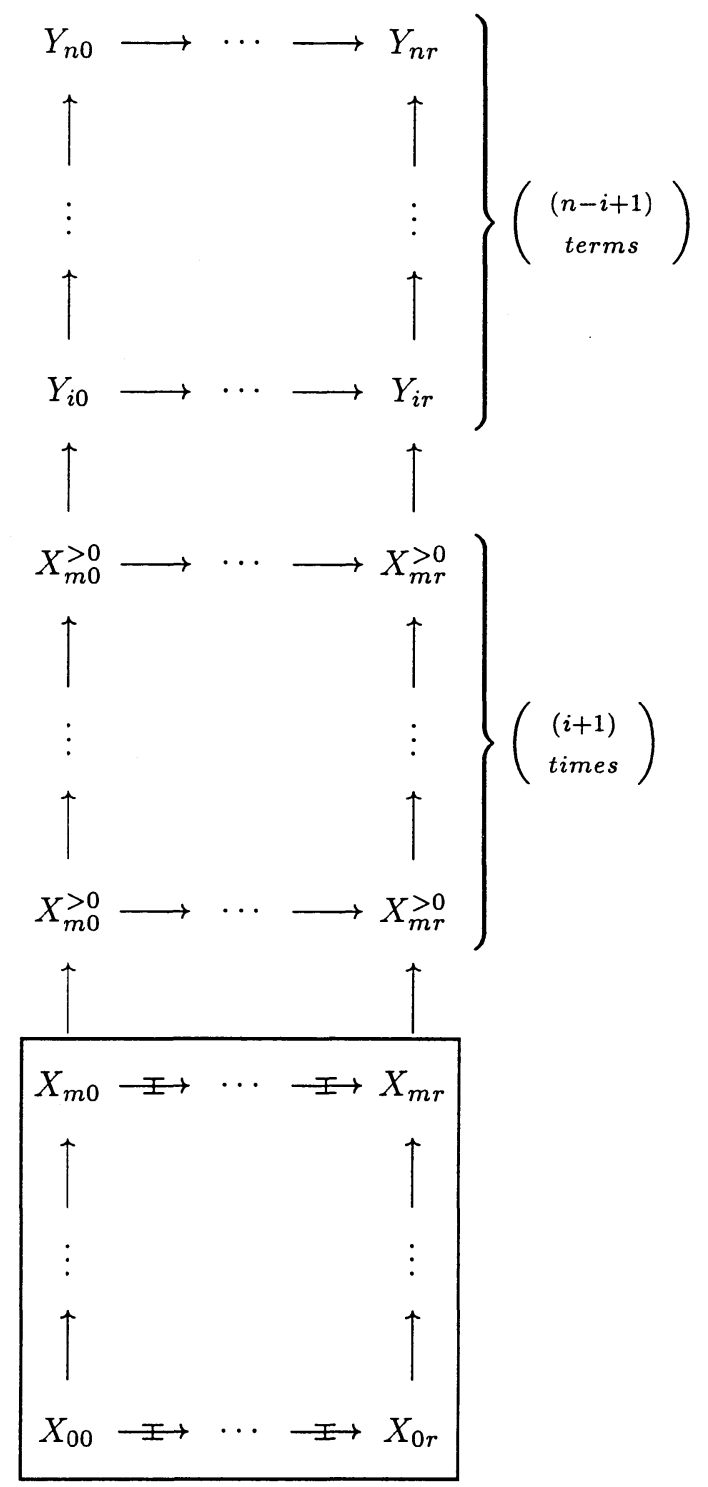

and this homotopy would naturally go under the name 


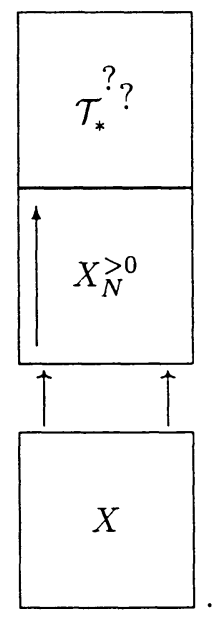

The reader should therefore note that, although our notation is similar in spirit to Quillen's, there are differences of detail. Our notation tells us not only that we are contracting to the initial object. It also tells us the shape of this initial object. The notation attempts to capture in a brief picture the key features of the homotopy; Quillen's notation is more geared towards specifying the homotopies by certain formal properties.

The problem with this homotopy is that it need not be a contraction. This is a point about which we very cavalierly skipped in the $K$-theory for triangulated categories $I$. One reason I felt entitled to be so cavalier is that this subtle point does not affect $G r$. In $K$-theory for triangulated categories $I$ we had been studying mostly the properties of $G r$, and the proof of Theorem I.4.8.

The homotopy (which is perfectly well-defined) connects the identity map with the map sending the simplex $s_{n}$ to 


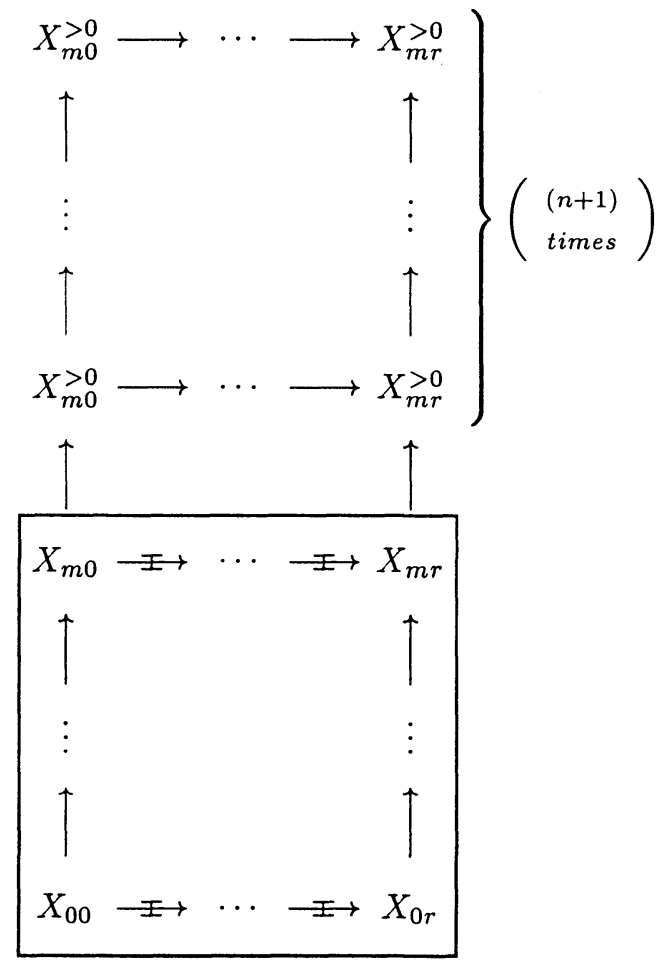

And the precise difficulty is that the differential, which is, of course, well defined in terms of

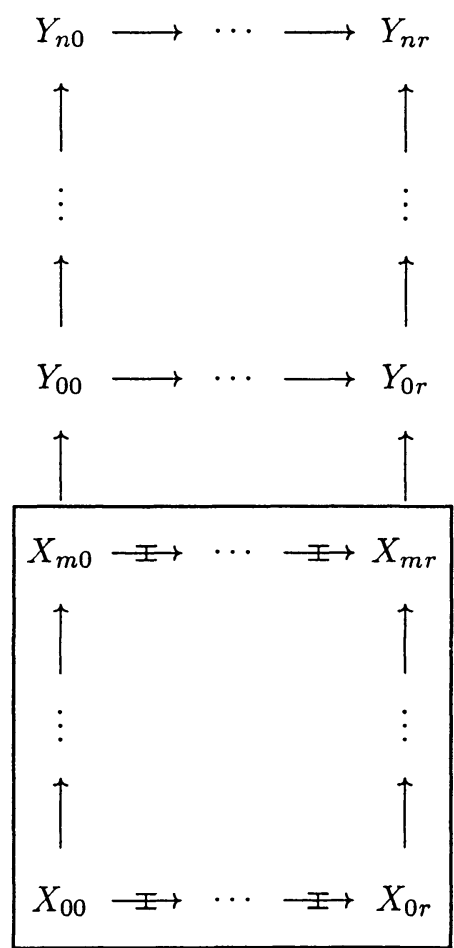


depends on the $Y^{\prime} s$. Given an

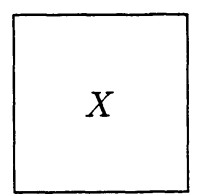

one can (maybe) complete to a simplex

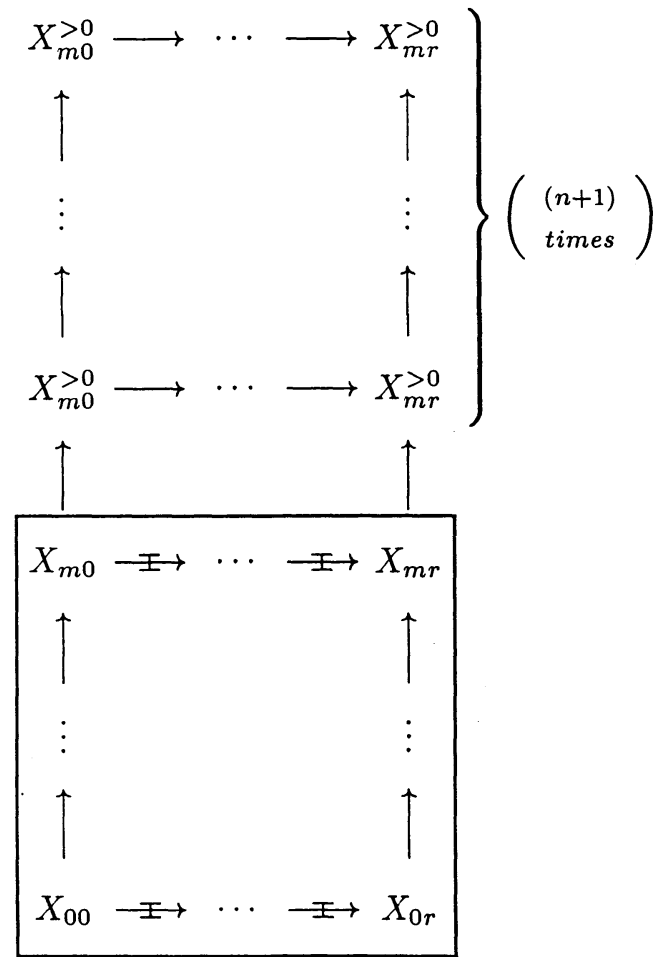

but the differential is not unique. The choice of differentials is, of course, implicit in the choice of differentials $Y_{r n} \rightarrow \Sigma X_{00}$. Thus the homotopy

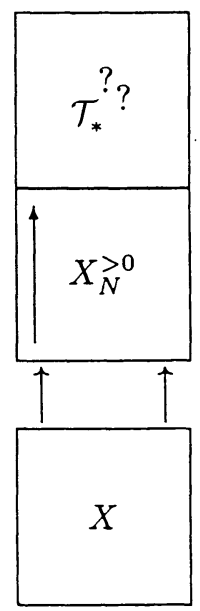


is not a contraction. It contracts each component of

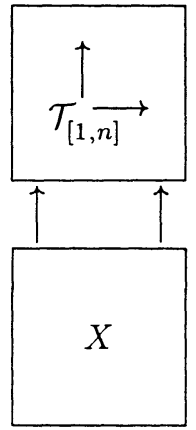

to a point, but in fact the simplicial set

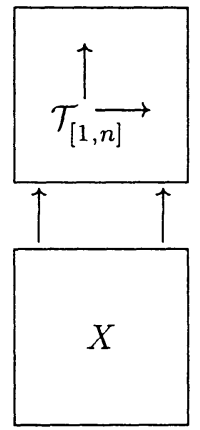

is discrete.

REMARK 1.1. This point is so important that the author feels he should explain it in detail, although the reader can undoubtedly provide the detail unaided.

Suppose we are given a map $f: W \longrightarrow Z$ in $\mathcal{T} \geq 0$. Suppose that $g: A \longmapsto W$ is an inclusion of an object $A \in \mathcal{T}_{[0,0]}$ into $W$. Suppose furthermore that $f \circ g=0$. Then of course $f$ factors as $W \longrightarrow \frac{W}{A} \longrightarrow Z$. But in fact the factorisation is unique. The reason is that the ambiguity comes as follows. We have a triangle

$$
A \longrightarrow W \longrightarrow \frac{W}{A} \longrightarrow \Sigma A
$$

to which we can apply the cohomological functor $\operatorname{Hom}(-, Z)$. From the long exact sequence we deduce that the ambiguity in the map $\frac{W}{A} \longrightarrow Z$ must come from a map $\Sigma A \longrightarrow Z$. But $\Sigma A$ is in $\mathcal{T}^{<0}$, whereas $Z$ is by hypothesis an object of $\mathcal{T} \geq 0$. Therefore, by I.6.0.2, the only map $\Sigma A \longrightarrow Z$ is the zero map. Hence the uniqueness of the factorisation.

However, in the above we were attempting to factor the given map $X_{m r} \rightarrow \Sigma X_{00}$ through some $X_{m r}^{\geq 1} \rightarrow \Sigma X_{00}$. And the problem is precisely that even if $X_{00}$ is an object of $\mathcal{T} \geq 0$, the object $\Sigma X_{00}$ will tend not to be.

The fact that these maps are differentials does not help, but it also does not hinder. If the truncation of the differential is given by some extra structure on the simplex, the structure will usually also guarantee that the truncation is a differential of the obvious truncated triangle.

The reader should ponder this point carefully now. If $\mathcal{T}=\operatorname{Gr}(\mathcal{A})$, then in fact the coherent differential exists and is unique. In the reasoning of Remark 1.1, this is 
because the map $\frac{W}{A} \longrightarrow \Sigma A$ always vanishes in $G r$. Therefore in Sections I.7 and I.8 we were quite cavalier about this homotopy, and its various cousins. See, for example, Lemma I.8.1, the proof that $f_{1}$ is a homotopy equivalence. In the case where $\mathcal{T}$ is a triangulated category, there are two ways to get around the difficulty: One can postulate that a differential $X_{\bar{m} r}^{\geq 1} \rightarrow \Sigma X_{00}$ is given as part of the simplicial data (we will have occasion to do this in Section 3). Alternatively, one can try to avoid trouble by working only with large simplicial sets. For example, in

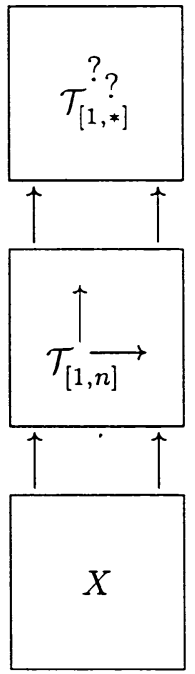

the homotopy

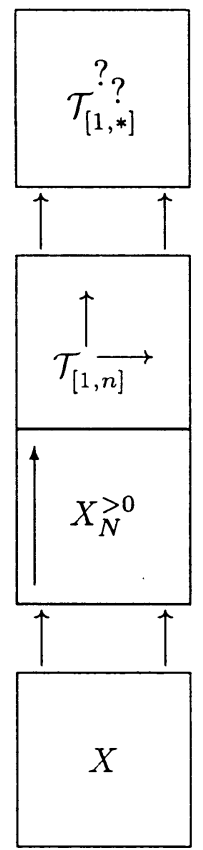

does give a contraction, as the notation might suggest. The point is that, because we are already given a factorisation of the differential $X_{m r} \rightarrow \Sigma X_{00}$ through an object in $\mathcal{T}^{\geq 1}$, (choose any one in the top rectangle,) the factorisation of $X_{m r} \rightarrow \Sigma X_{00}$ as $X_{m r} \rightarrow X_{\bar{m} r}^{\geq 1} \rightarrow \Sigma X_{00}$ is unique and unambiguous.

To illustrate what might be a practical application, consider the simplicial set 


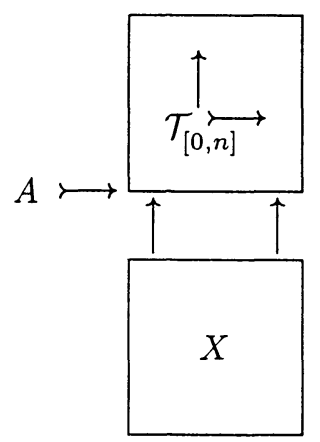

where $A$ is a fixed object of $\mathcal{T}_{[0,0]}$, injecting into all the objects above it. A simplex is therefore a diagram

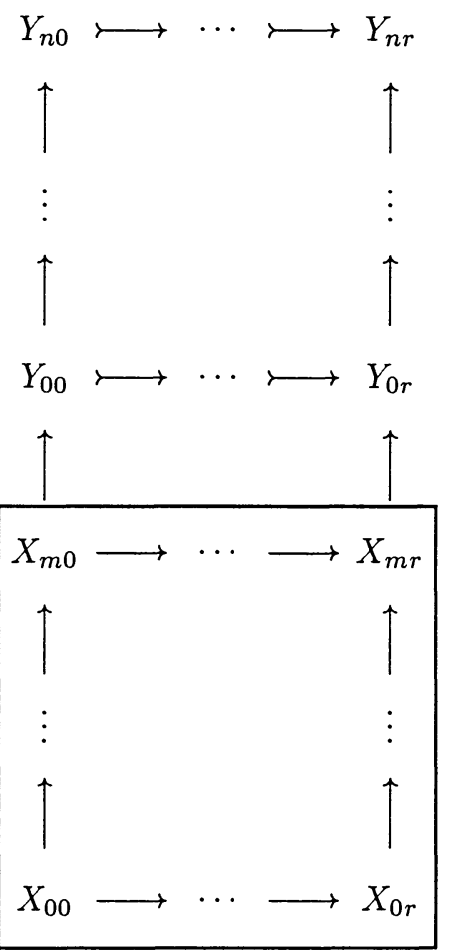

together with monos $A \longmapsto Y_{i j}$ for all $i$ and $j$, so that all the maps in the $Y$-rectangle are $A$-maps (except the differentials, of course). There is a simplicial map

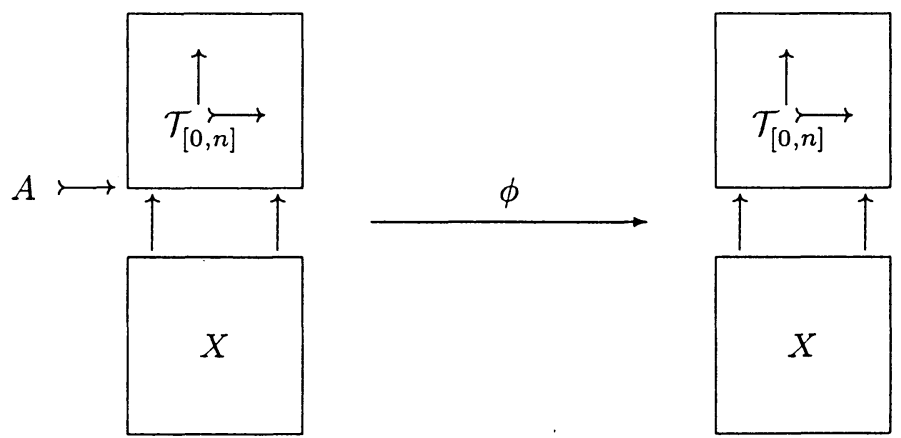

which forgets the inclusion from $A$. One could imagine a homotopy, starting with the 
map $\phi$. The homotopy would be denoted something like

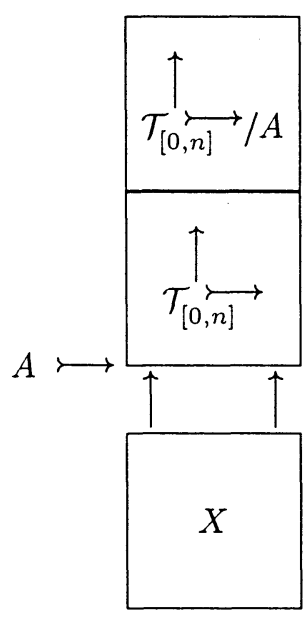

and its cells should be 


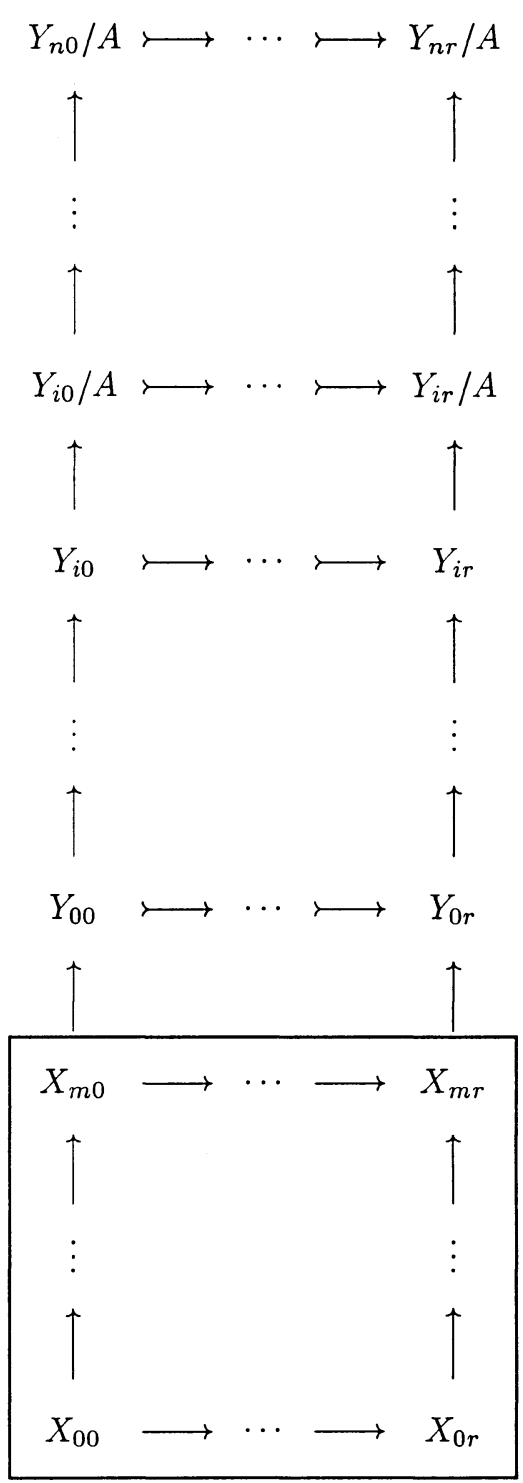

And, as the reader undoubtedly guessed, the problem is the non-uniqueness of the differentials $Y_{n r} / A \rightarrow \Sigma X_{00}$. However, this problem disappears completely in the simplicial set 


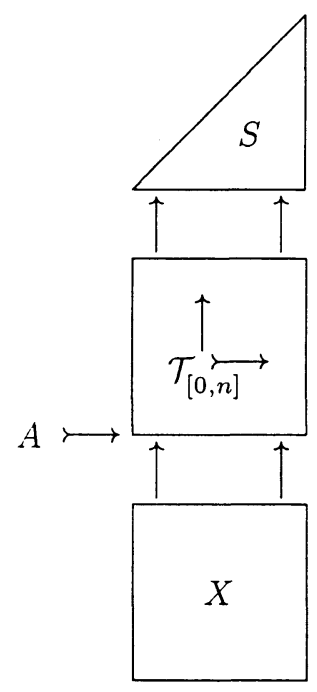

Here the homotopy

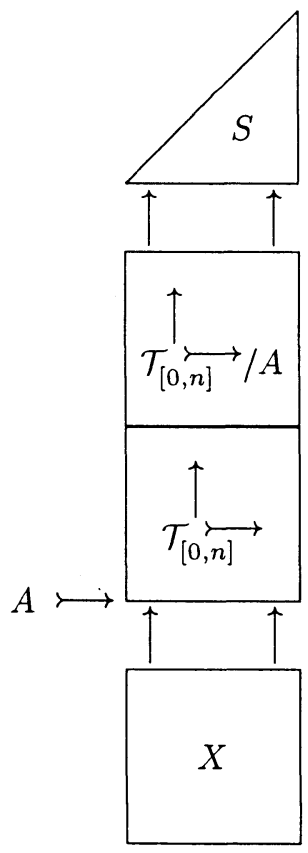

is well-defined. An example of this, albeit with an extra twist, may be found in Lemma I.7.6.

Another very trivial homotopy is the addition (= direct sum) of a constant object. Let 


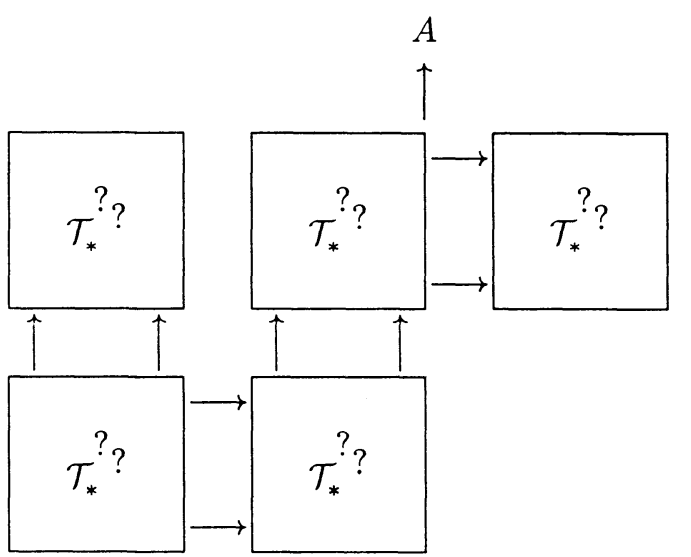

be the obvious simplicial set, whose simplices are simplices in

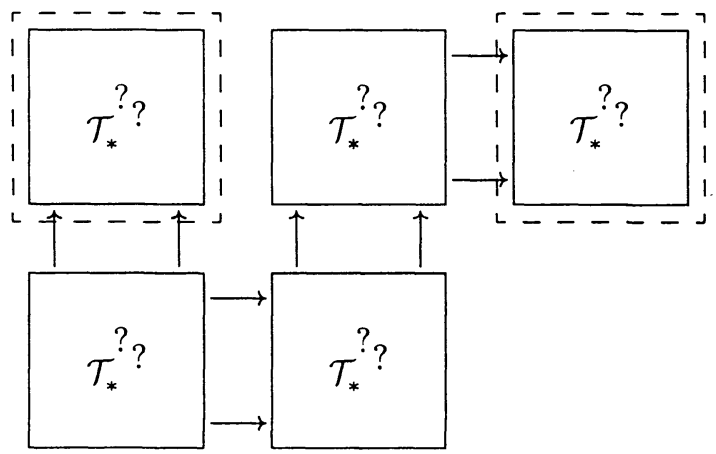

but where, in addition, every object outside the two highlighted boxes comes equipped with a mapping to the object $A \in O b(\mathcal{T})$, and all the morphisms (excepting the differentials) are $A$-maps. If the reader wishes he can, as the notation suggests, simply think of a map to $A$ being given from the top right hand corner of the indicated square, and the face maps induce composition.

Then there is a homotopy, which we will denote

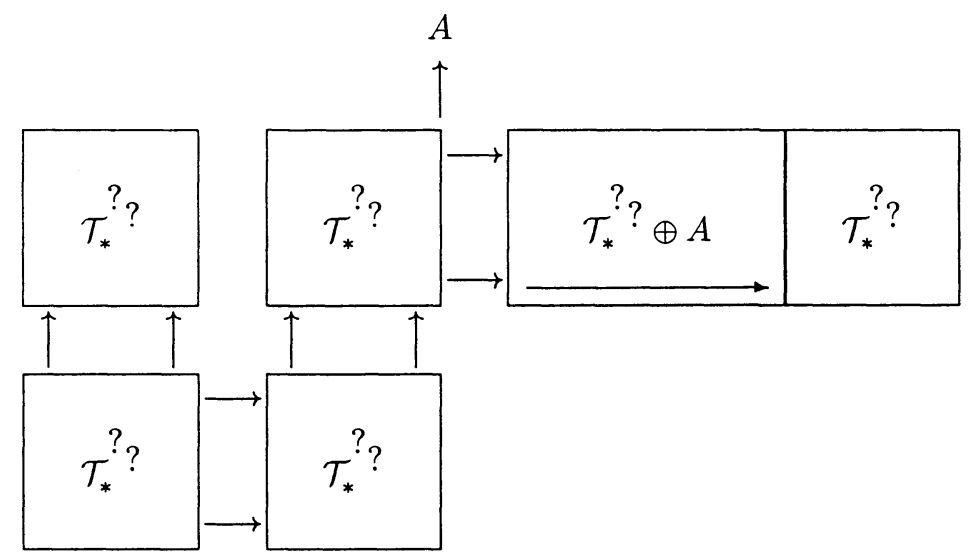

which simply adds $A$ to every object in the right hand square.

We have now discussed the three trivial homotopies which arise: $t$-structure truncations, contractions to initial and terminal objects, and direct sums. It is time to turn our attention to the non-trivial homotopy. 
The one non-trivial homotopy assumes that we have, in some part of our simplicial set, the diagram

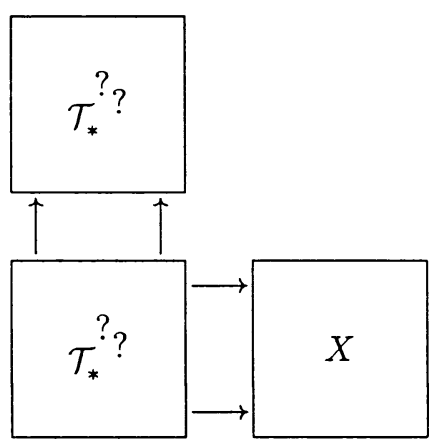

A simplex is then a diagram

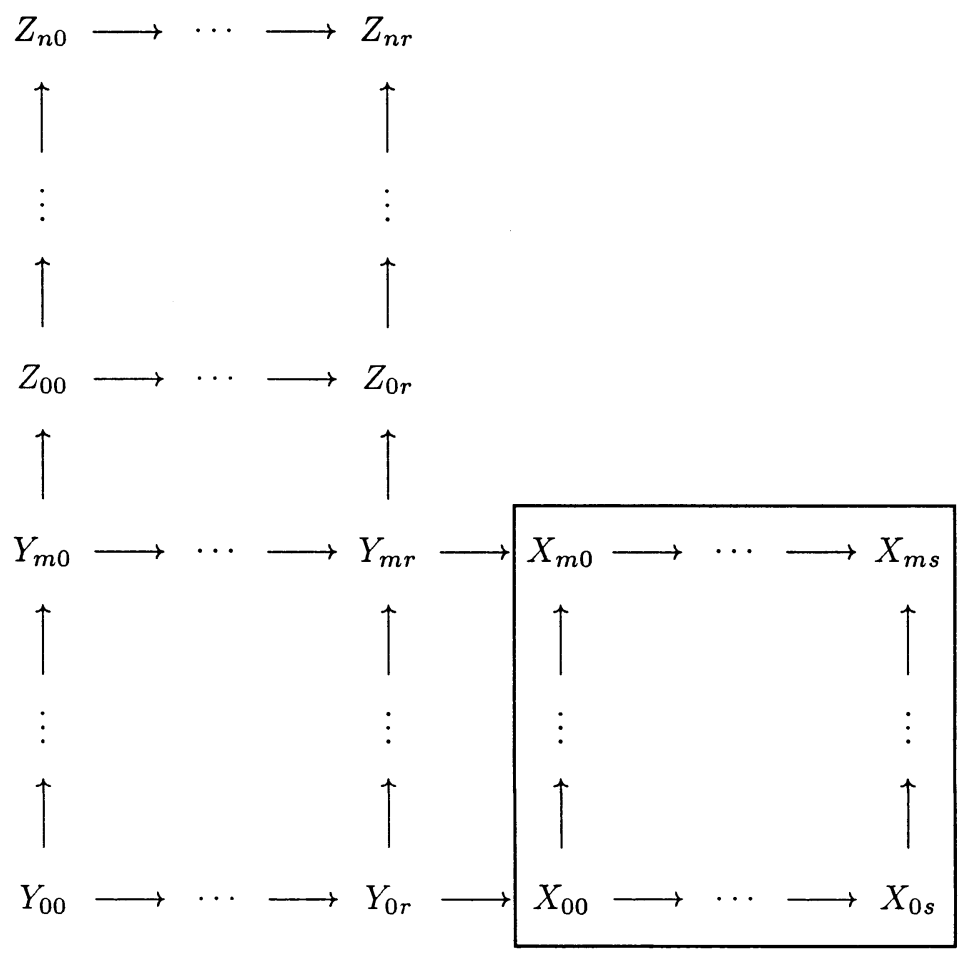

Since the $X^{\prime} s$ are fixed, it makes sense to write a homotopy taking the above simplex to simplices of the type 


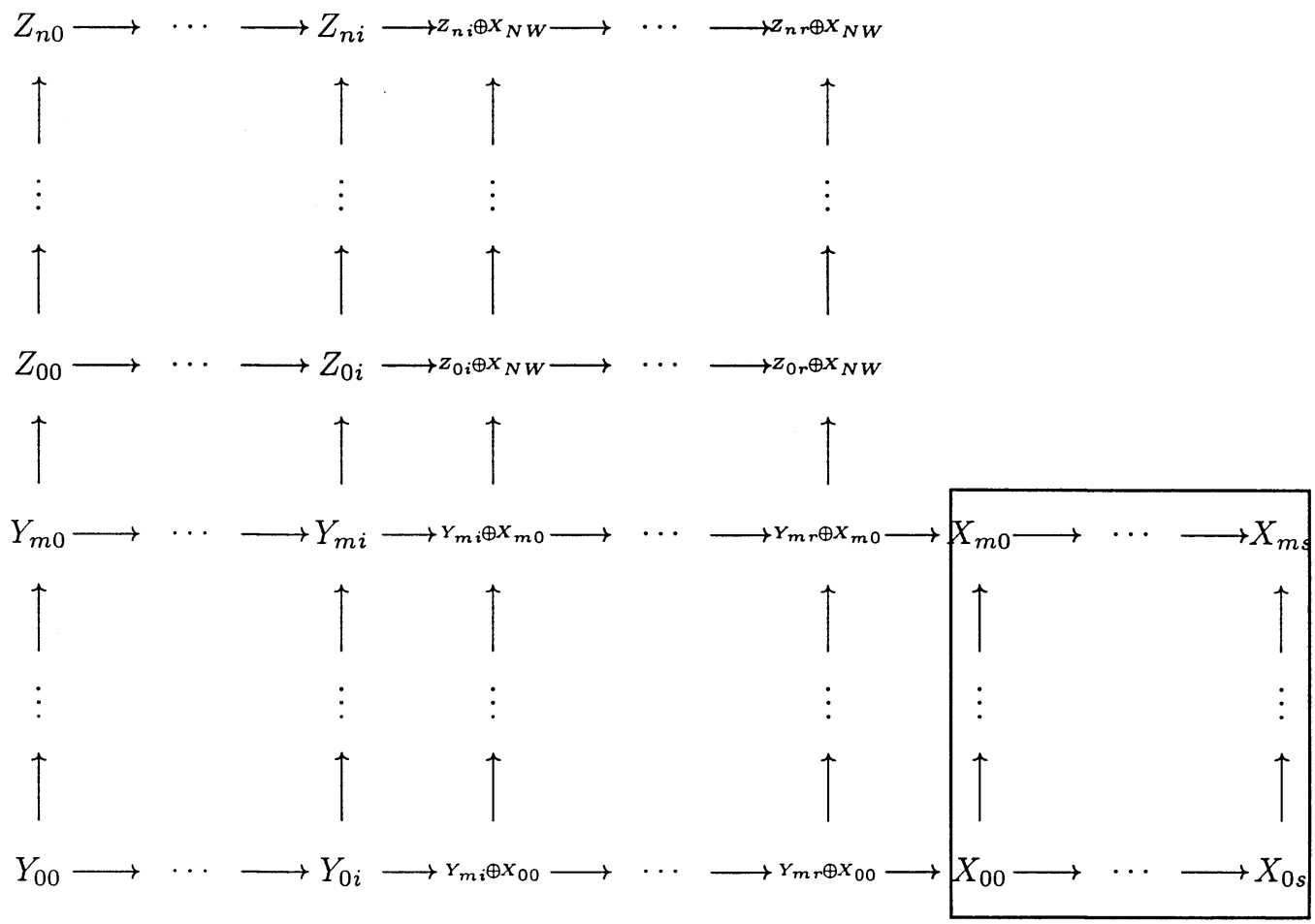

Our notation for this homotopy is

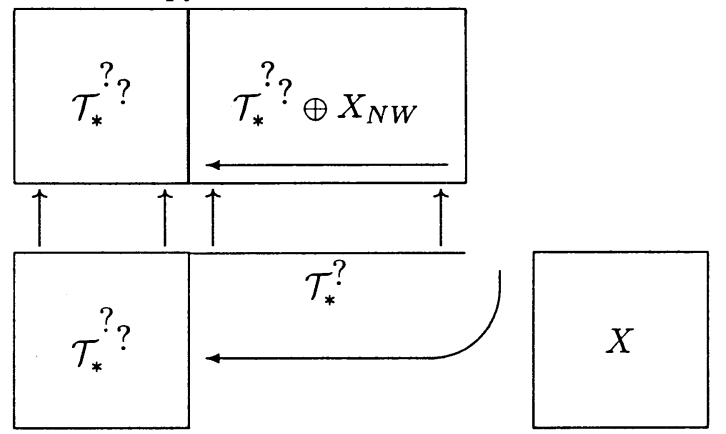

As before, $X_{N W}$ is intended to indicate the North-West corner of $X$, i.e. $X_{m 0}$. The end result of this homotopy is a simplicial map we denote

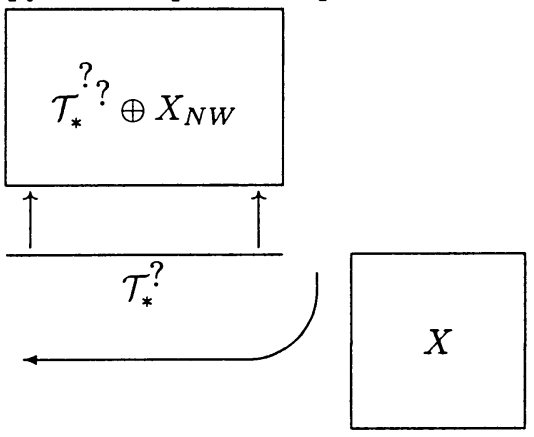

We remind the reader that the arrow indicating the direction of the homotopy has disappeared from the top square, but not in the bottom left hand corner. The curved 
arrow that remains in the notation is intended to remind us that what fills this square is made up of $X_{W}$ as well as the line at the top. The reader saw the homotopy, in precisely this form, in Lemma I.8.7.

As always, it does no harm if we concatenate some additions to our simplicial sets. To treat a suitably general case, the reader might care to show that the homotopy on the simplicial set

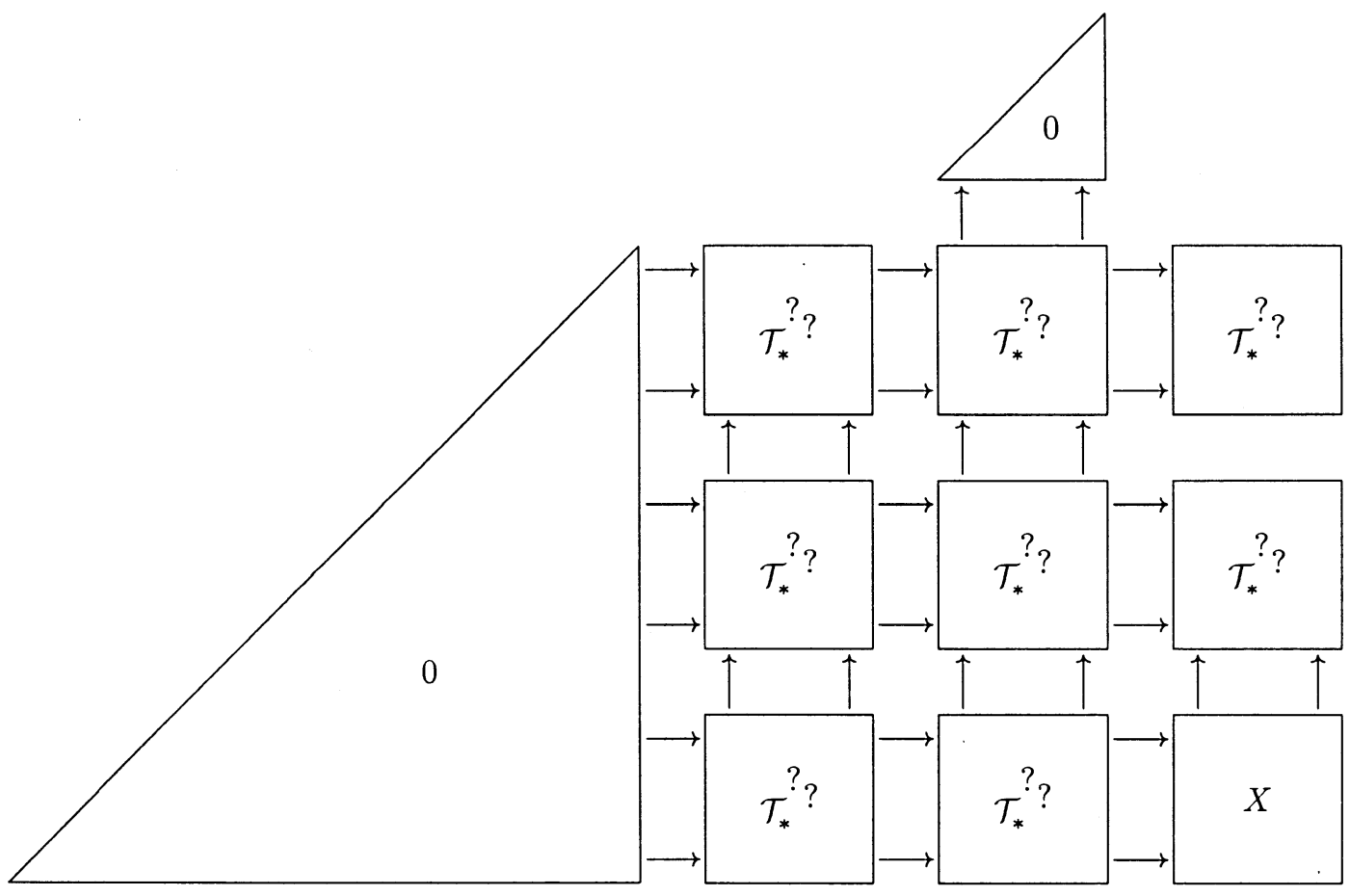

which in our notation would be abbreviated 


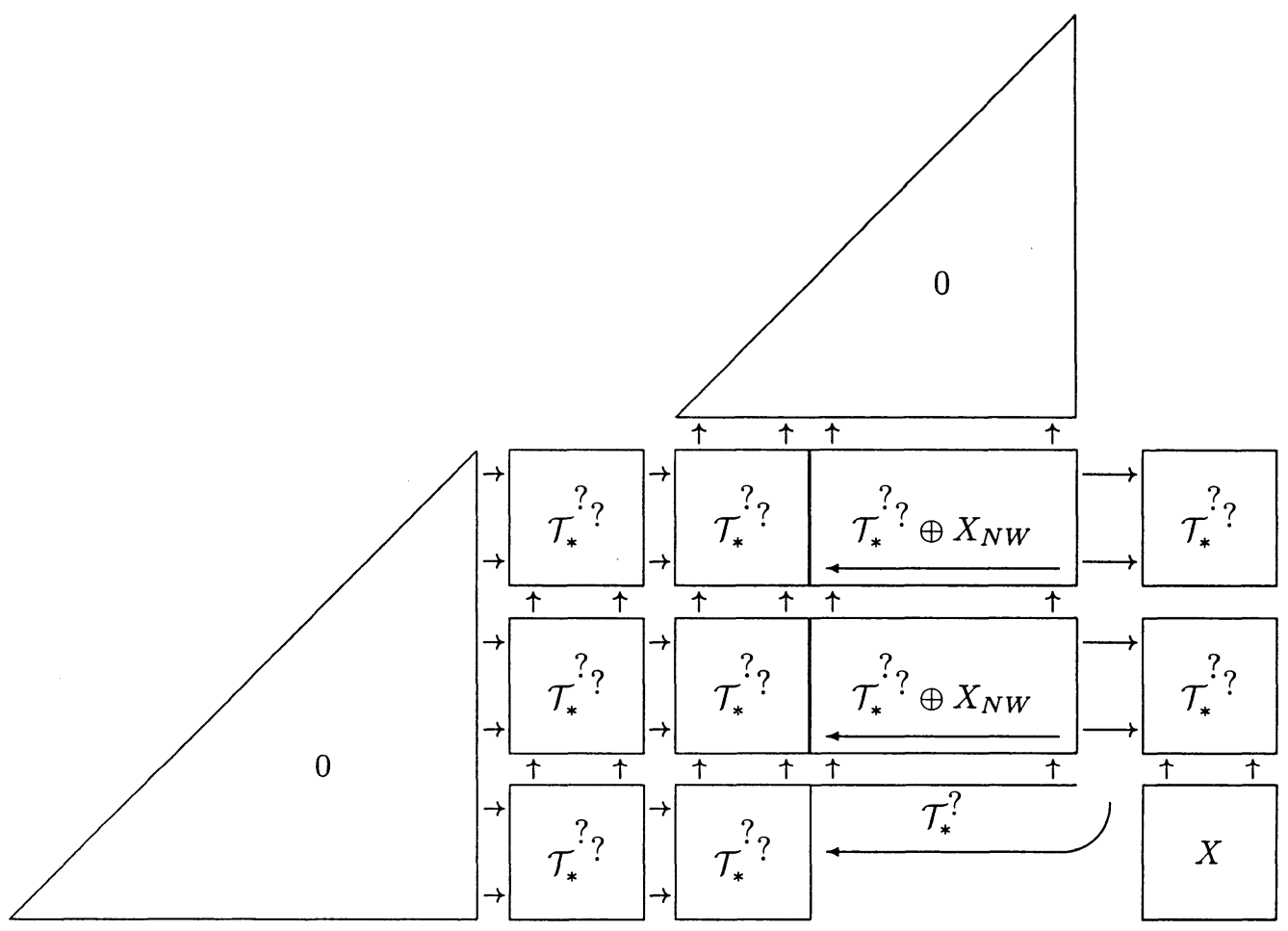

makes sense in the obvious way, with all but the middle column of cells unaltered by the homotopy. Checking this amounts to establishing that certain rectangles are Mayer-Vietoris. The only non-trivial ones are those where one of the columns lies in the action region of the homotopy. This gives a finite list of possible squares to check, most of which are trivial. There is a detailed discussion of this in Section III.1. The reader is also referred to Remark I.5.3.

It is, of course, possible to adorn the simplicial set even more, gluing yet more pieces that come nowhere near the homotopy. The reader can amuse himself by considering

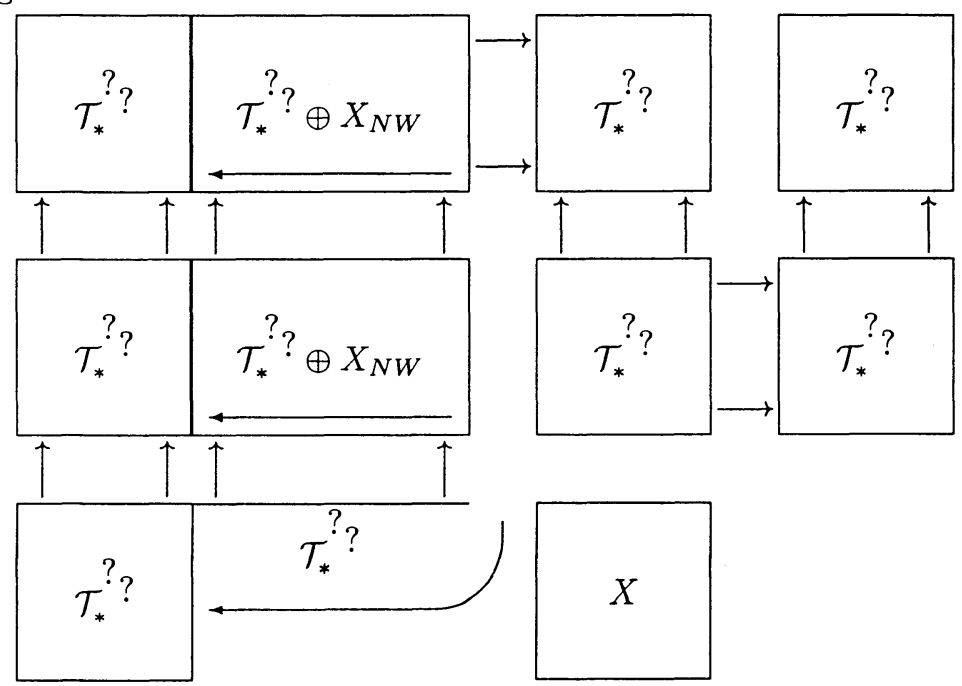

It is completely clear that no terms from the two rectangles on the right, or from 
the center rectangle, can be part of a candidate $M-V$ square anywhere near the homotopy. Thus, the adornment is harmless.

I solemnly promise that in this article, when we apply the homotopy, it will be to a (possibly subdivided) small part of the prototype diagram I just made the reader check. Deleting part of the diagram can only prevent difficulties; there are fewer $M-V$ squares to check. Subdividing is harmless. Since the homotopy is well defined on the prototype, it must be well defined on any part (at least in the sense that all the squares claimed to be $M-V$ really are).

Of course, it goes without saying that the author will feel free to apply the transpose and duals of the homotopy.

The prototype application of the homotopy above is the following.

PRototype QUASIFIBRATION 1.2. The map
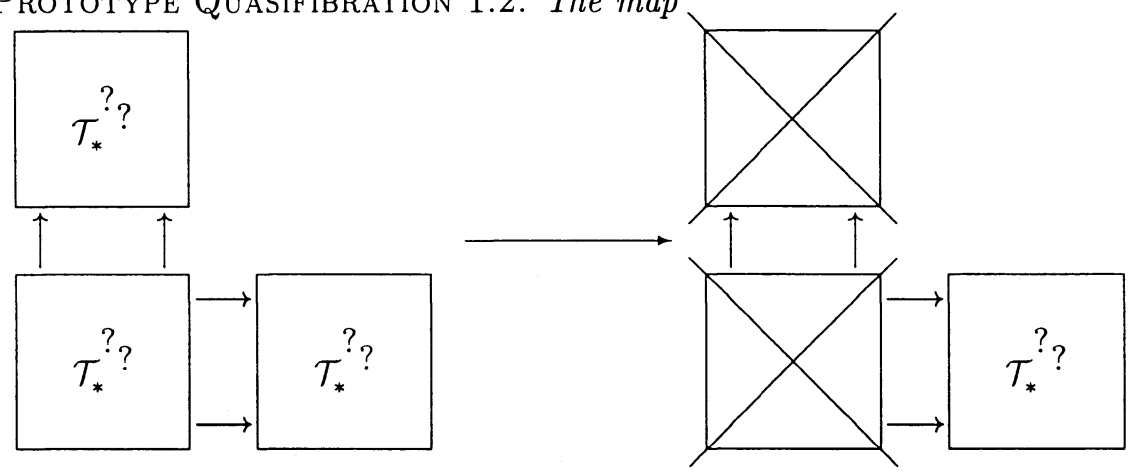

is a quasifibration. (Example: Lemma I.8.7.)

Prototype proof. We need to study the fiber, denoted

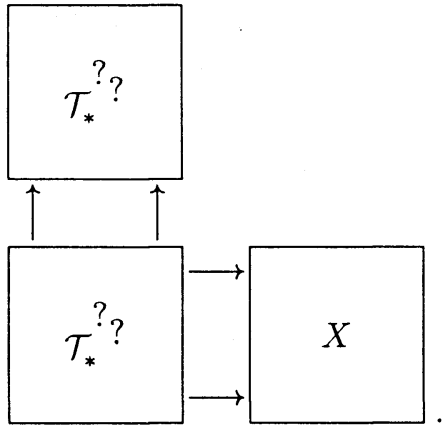

Apply first the homotopy

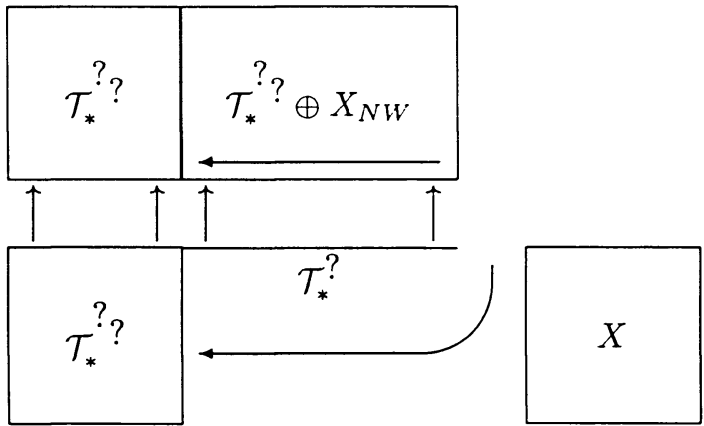

to establish that the identity on 


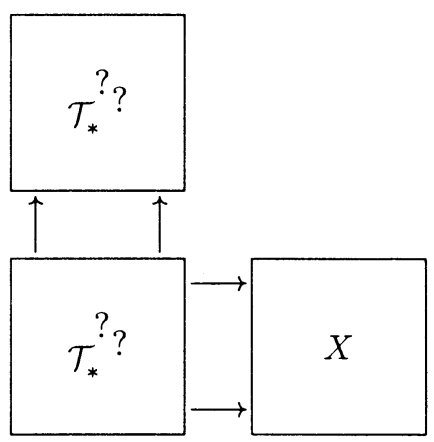

is homotopic to

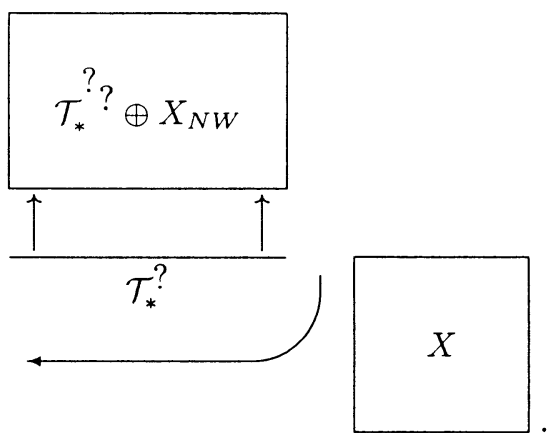

Now apply the homotopy

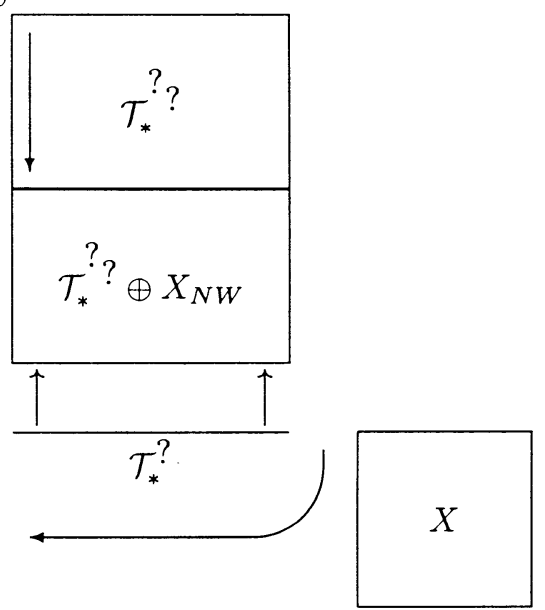

to show that the identity on

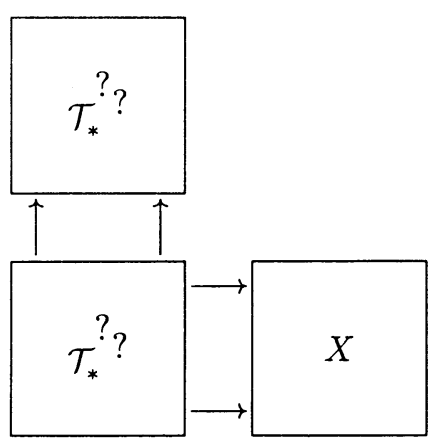


is in fact homotopic also to

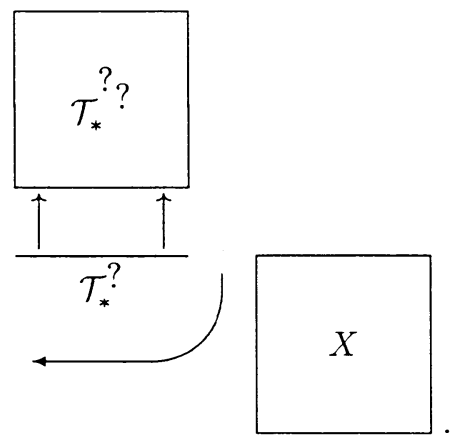

Of course, this map factors as

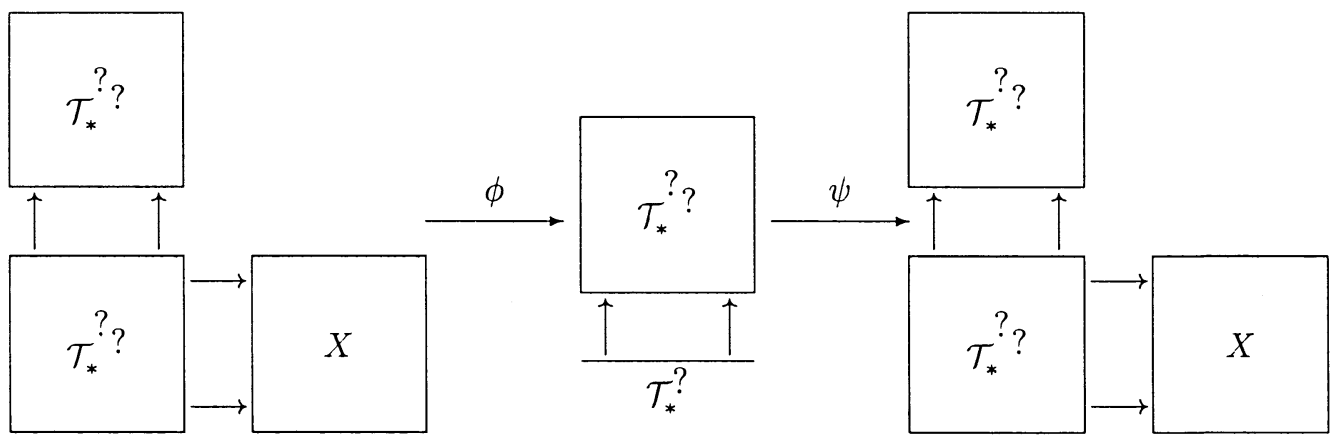

In other words, $\psi \circ \phi$ is homotopic to the identity. Clearly, $\phi \circ \psi$ is just the translation in the $H$-space structure of

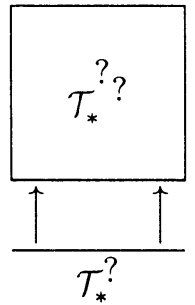

by the 0 -cell

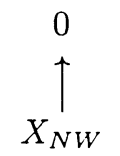

If we are lucky, this will be homotopic to the identity (for instance, if the $H$-space is connected). If $\partial$ is a face map on

$$
X
$$

then the composite 


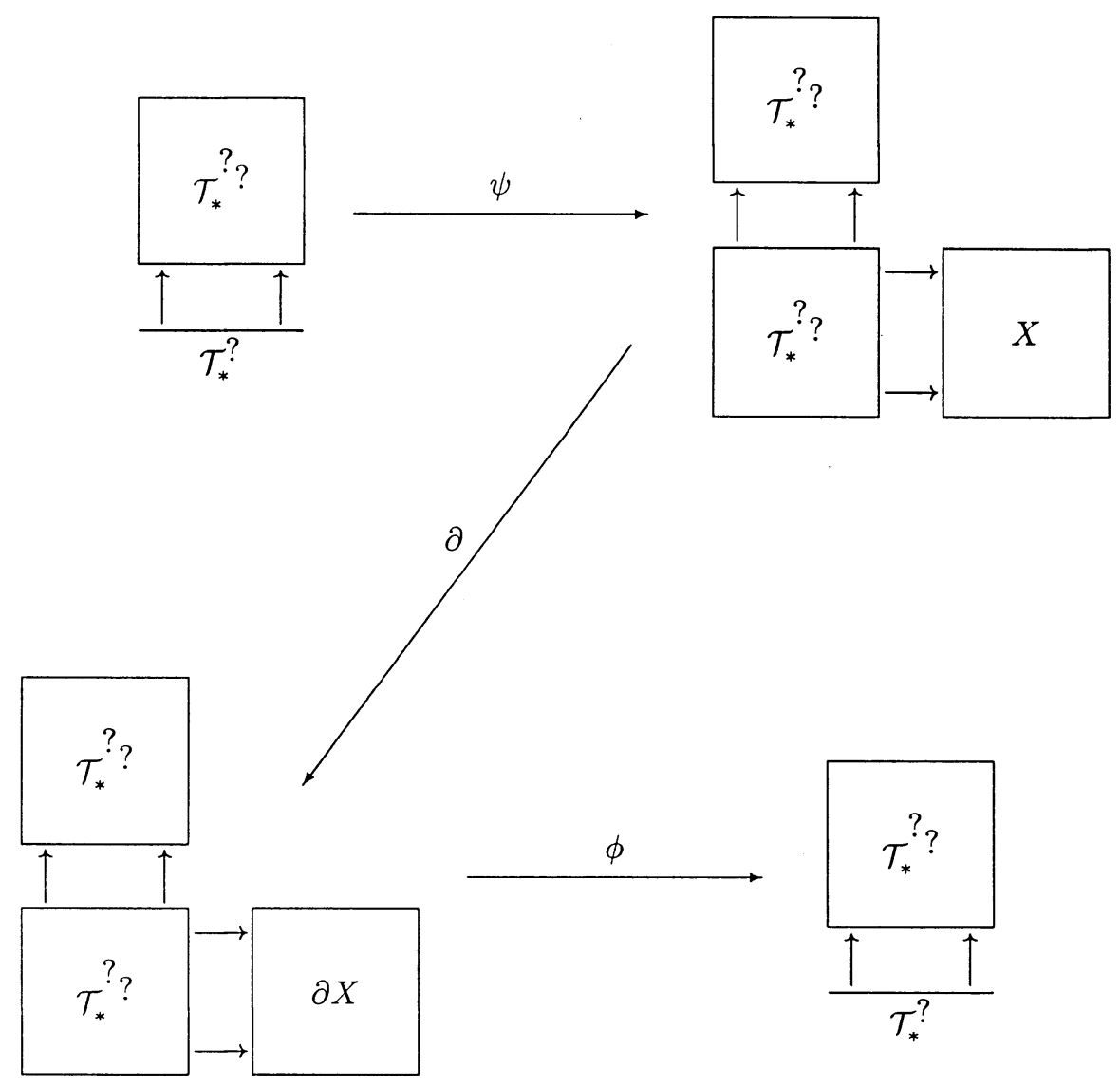

is translation in the $H$-space structure with respect to the zero cell

$$
\begin{gathered}
0 \\
\Upsilon_{i j}
\end{gathered}
$$

for some $(i, j)$ which depend on $\partial$.

God willing, this is also invertible, and thus $\phi, \psi$ and $\partial$ must all be homotopy equivalences. Hence the quasifibration assertion.

The proof of Lemma I.8.7 is an example that works. It is perhaps a good idea to give an example that fails.

Cautionary Example 1.3.

Perhaps a good illustration of the troubles that may arise is given by the discussion in the Motivation of Section I.8. Following our prototype quasifibration, we would expect to show that 

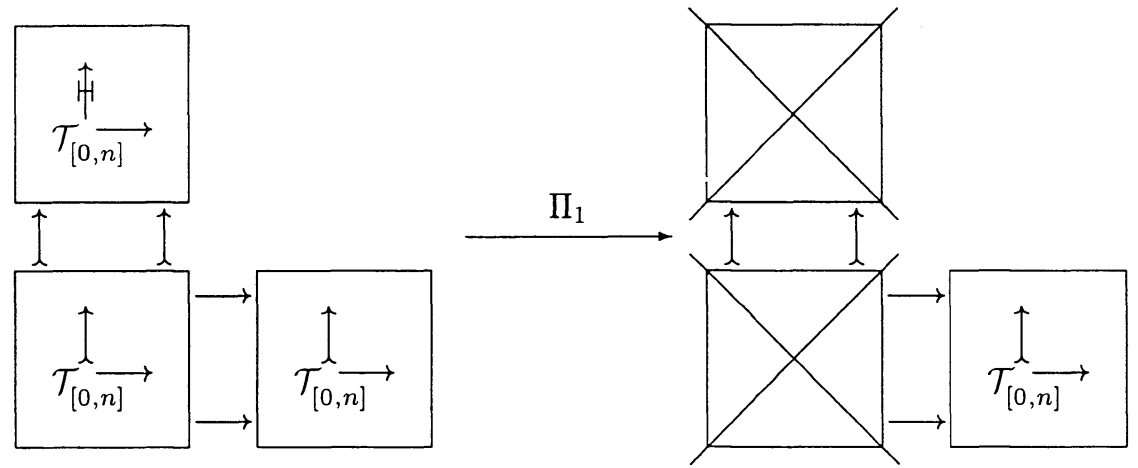

should be a quasifibration. Let us now follow the prototype proof. The first homotopy is

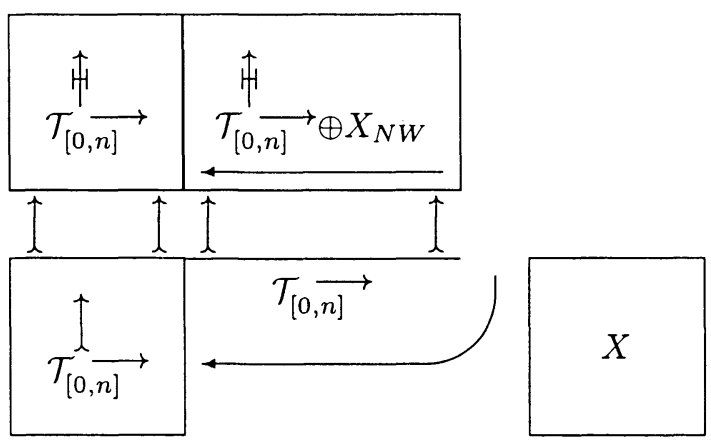

and there is absolutely nothing fishy about it. But, the second homotopy is

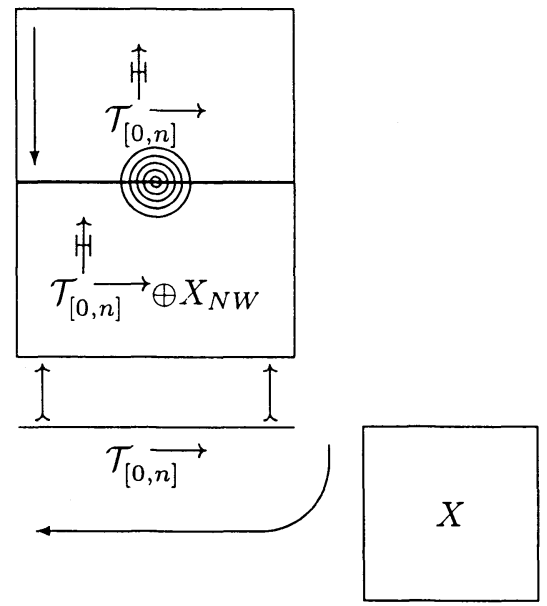

and there is plenty wrong with this homotopy; the trouble spot is highlighted with our trusty warning circles. In general, given an object $Z$ in $\mathcal{T}_{[0, n]}$, the projection $Z \oplus X_{N W} \rightarrow Z$ is not $I$, i.e. is not an isomorphism on $H^{0}$. It will only be an $H^{0}$-isomorphism in the lucky event that $H^{0}\left(X_{N w}\right)=0$.

This homotopy fails to be defined not because something asserted to be a triangle fails to be. It fails to be a homotopy becuse the candidate cells have wandered outside the simplicial set; their objects and morphisms do not satisfy the restriction hypotheses (cf Caution I.5.4).

The first homotopy showed that the fiber 


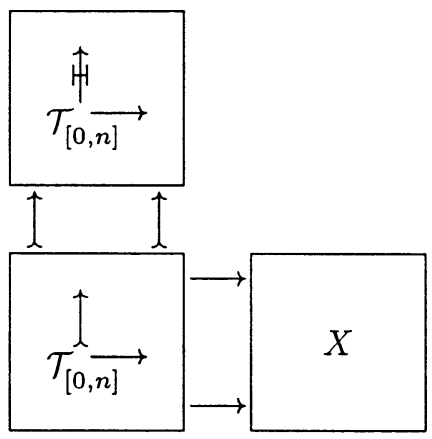

is homotopy equivalent to

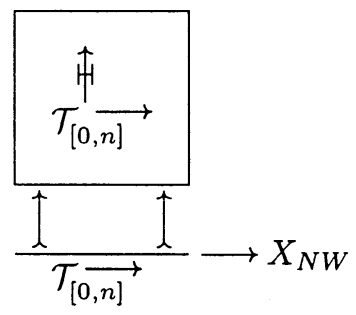

If $\mathcal{T}=\operatorname{Gr}(\mathcal{A})$, it is not difficult to show that this is in turn homotopy equivalent to

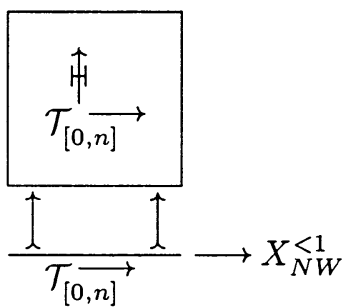

the point being that $X_{N W}=X_{N W}^{<1} \oplus X_{N W}^{>0}$, and we can homotope away the map to $X_{N W}^{>0}$; as was pointed out above, the problem with the second homotopy disappears if we assume the vanishing of the zero ${ }^{t h}$ cohomology. And $H^{0}\left(X_{N W}^{>0}\right)=0$.

But to prove $\Pi_{1}$ a quasifibration, we would have to show (among other things) that the projection:

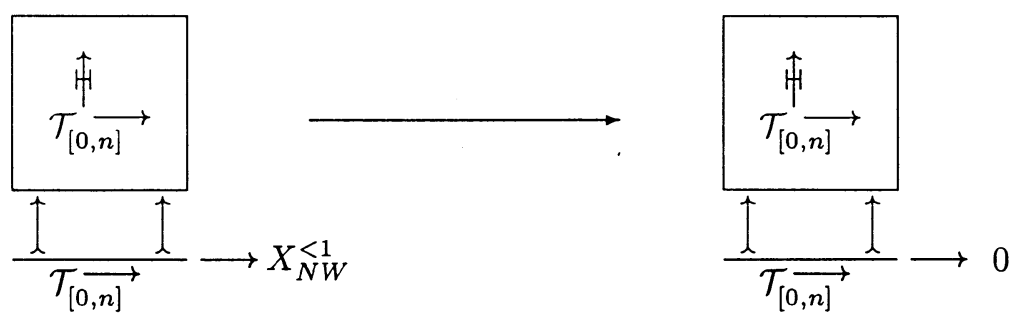

induces a homotopy equivalence.

Of course, once we know the entire proof of Theorem I.4.8, we also know that the right hand side is contractible, and hence by comparing the fake $H$-space structure on the left hand side with the action, as in the proof of Lemma I.7.11, we can deduce the contractibility of the left hand side. But by the time we know Theorem I.4.8 we hardly care whether $\Pi_{1}$ is a quasifibration. And as I said in the Motivation to the proof of Section I.8, I can prove directly that $\Pi_{1}$ is a quasifibration only in the 
special case where $\mathcal{T}=\operatorname{Gr}(\mathcal{A})$ and the construction is the one without differentials. Furthermore, the proof is dreadful. What is more, in Cautionary Example 1.5 we will make the case that the proof has to be dreadful.

The quasifibration of Prototype 1.2 is extremely stable under small perturbations. For instance, it can be varied to give:

PRototyPE QUASIFIBRATION 1.4. The projection

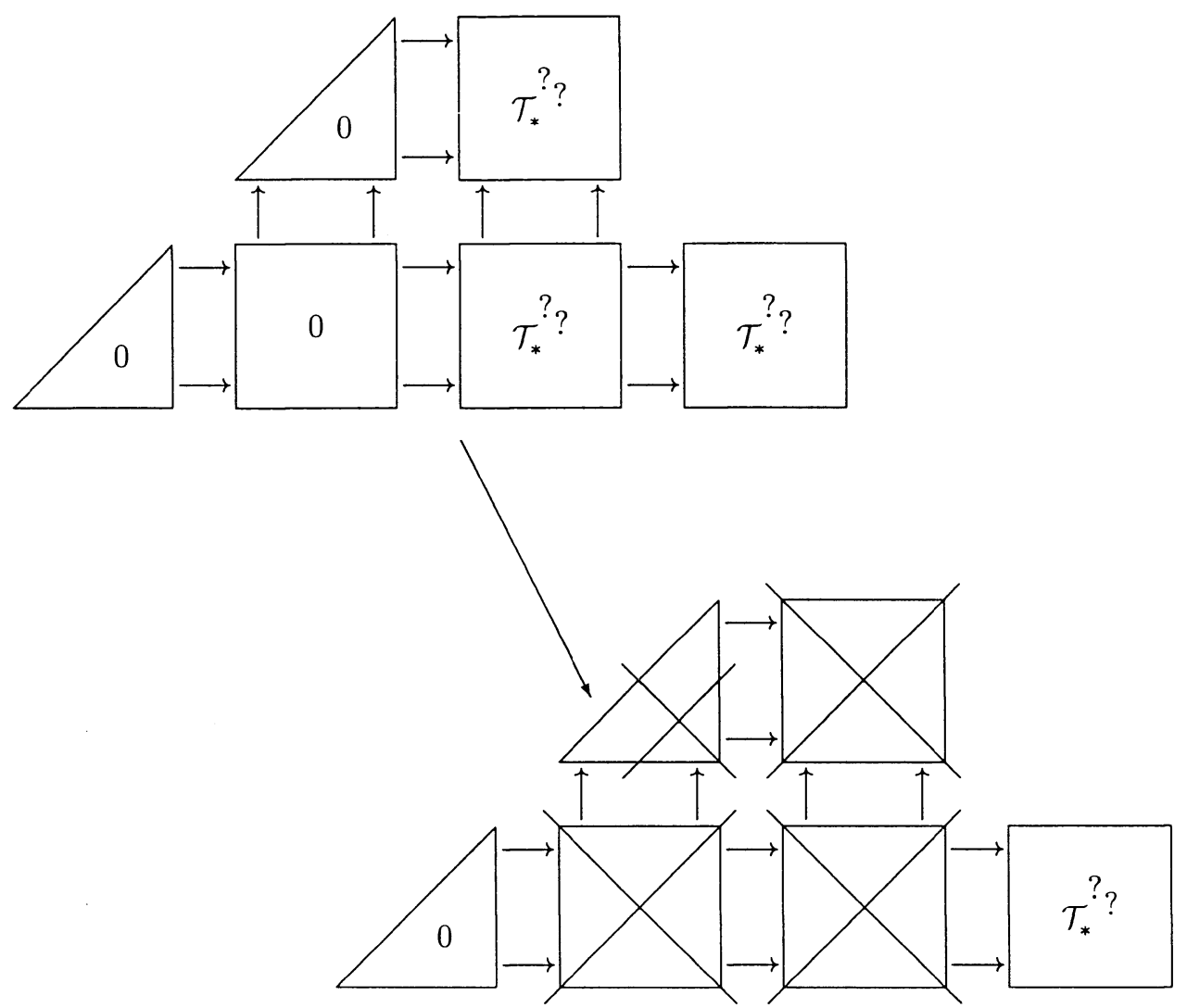

induces a quasifibration.

Proof. Once again, apply the homotopies

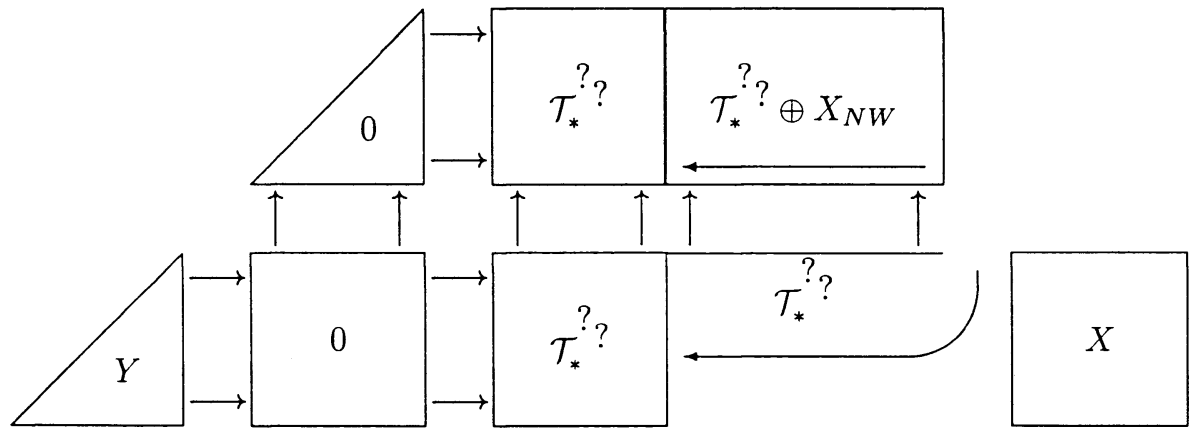




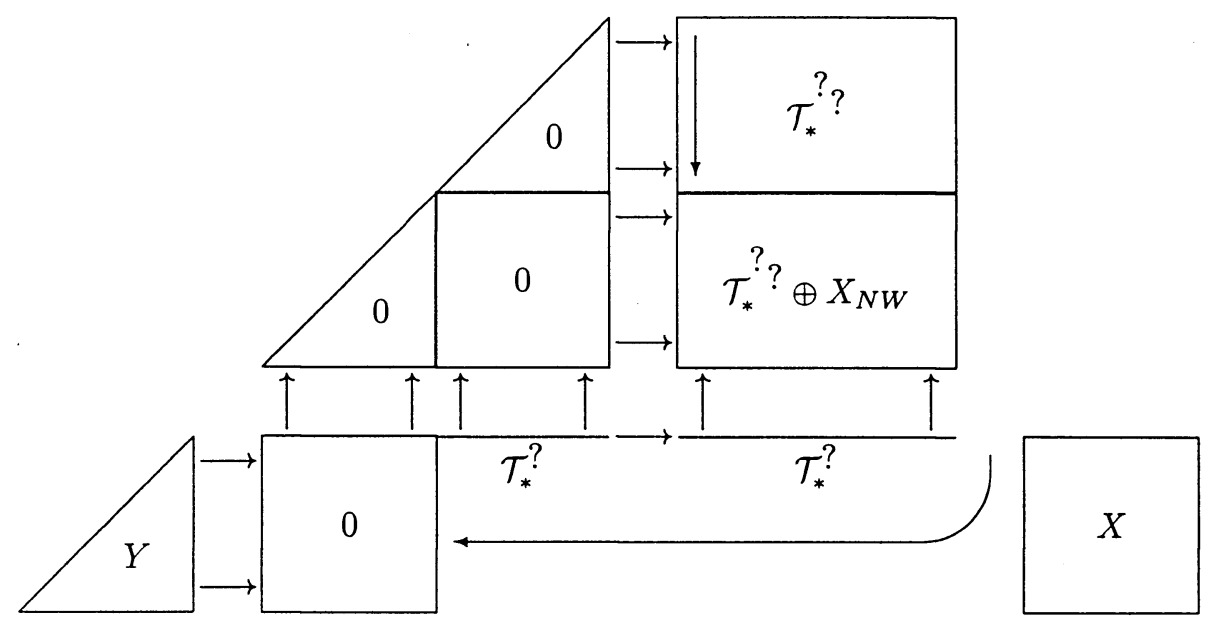

We leave it to the reader to compute the cells of these homotopies and show that they are well defined. We have not yet had occasion to use them in this article, but we will.

Thus the identity on

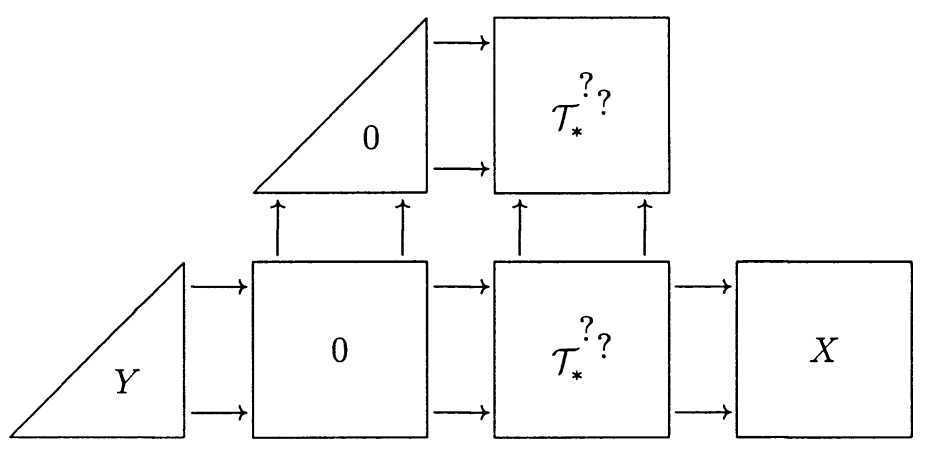

is homotopic to the map

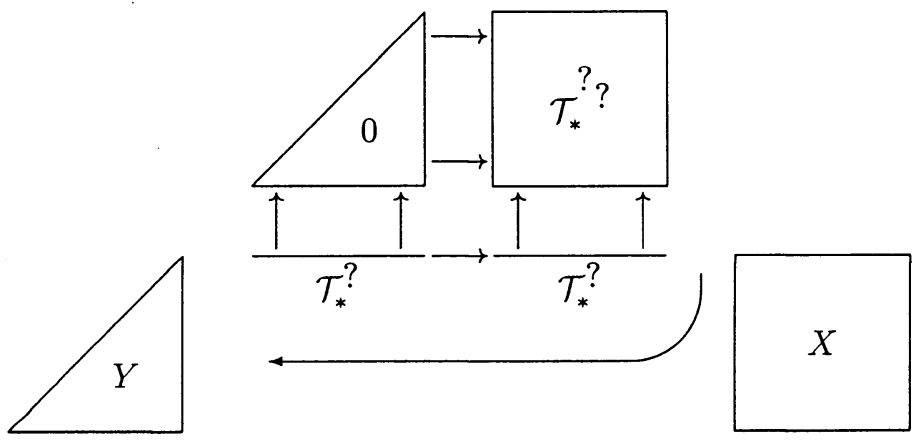

which factors as 

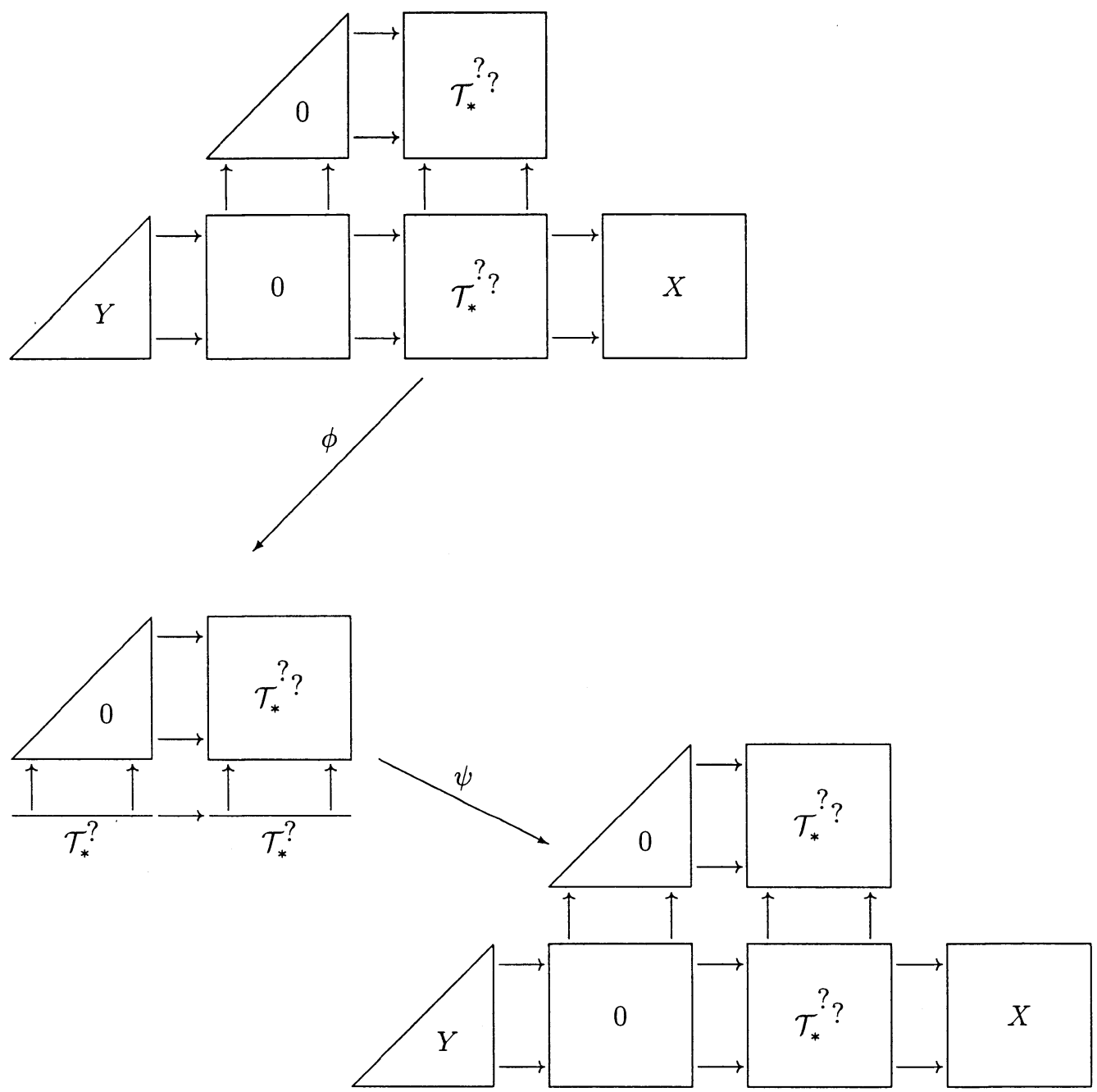

And, just as in Prototype Quasifibration $1.2, \phi \circ \psi$ is translation in the $H$-space structure with respect to the zero-cell

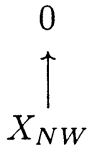

while for any face map $\partial, \phi \circ \partial \circ \psi$ is translation in the $H$-space structure with respect to

$$
\begin{gathered}
0 \\
X_{i j}
\end{gathered}
$$

God willing, these are both invertible, and hence $\partial$ is a homotopy equivalence.

Once again, let us study the analogue of Cautionary Example 1.3.

Cautionary Example 1.5. If we try to apply the above to the projection 

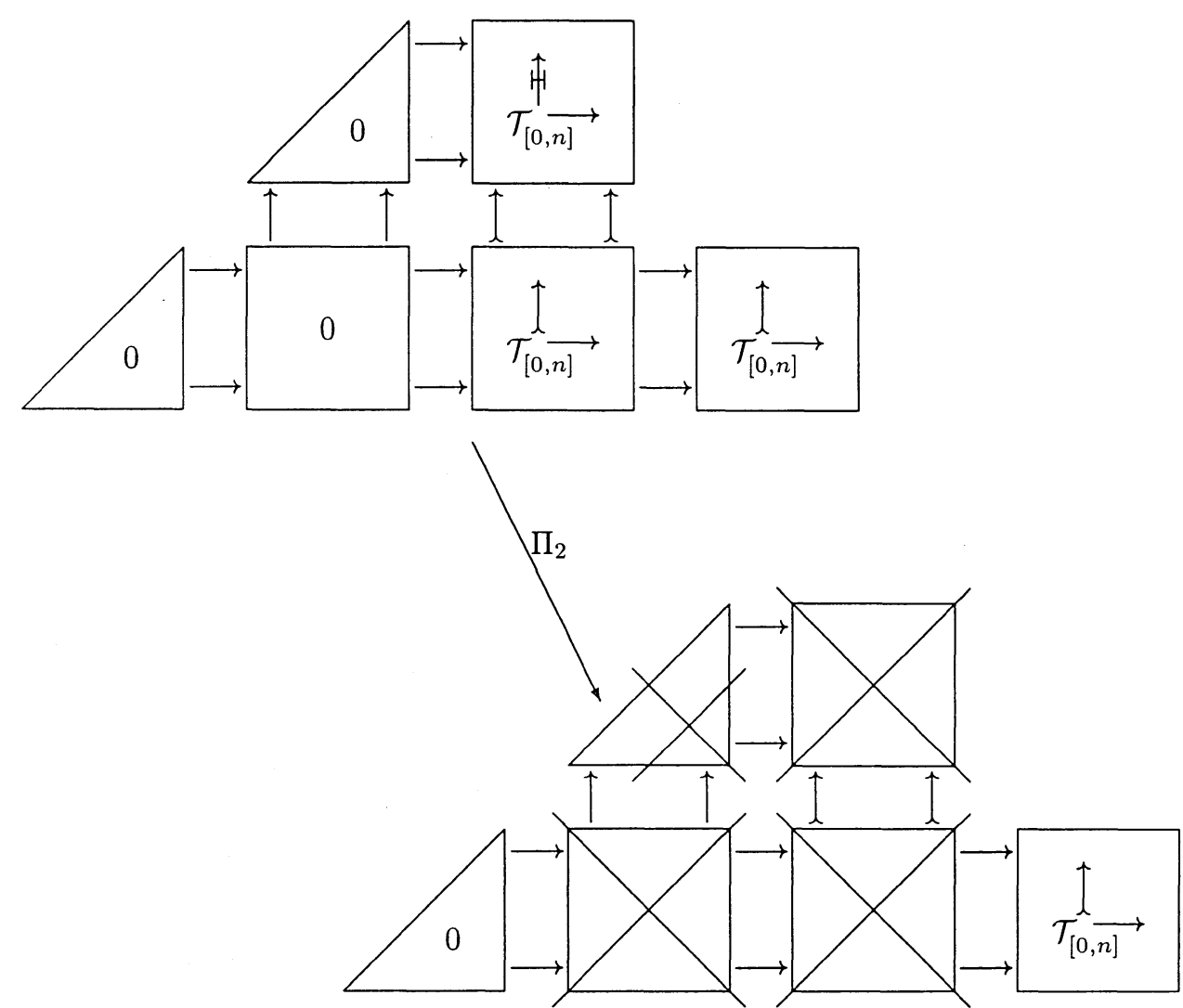

we would like to deduce that it is a quasifibration. Once again, we run into difficulties, essentially the same as in Cautionary Example 1.3. But now we wish to reason that the difficulties are genuine, not just a result of a faulty approach to the problem.

Suppose there were some simple, direct way to show that both $\Pi_{1}$ of Cautionary Example 1.3 and $\Pi_{2}$ of Cautionary Example 1.5 are quasifibrations. Since $\Pi_{1}$ and $\Pi_{2}$ are essentially the same map, the fibers would then be homotopy equivalent. Thus
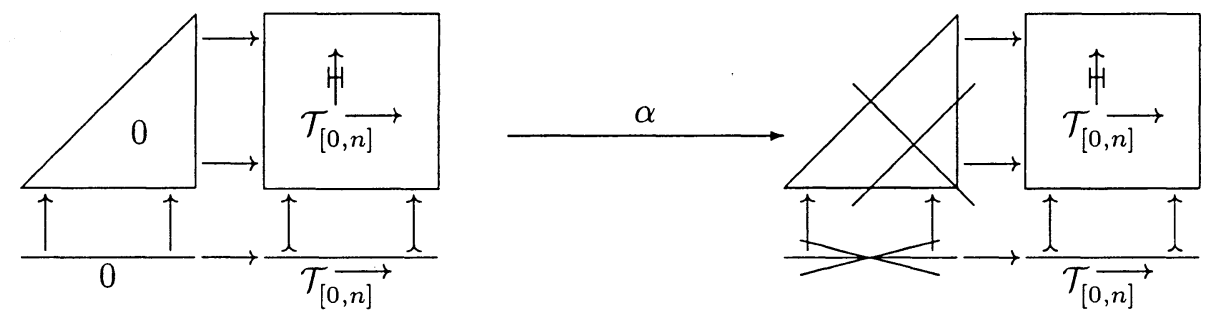

would be a homotopy equivalence.

But this would have serious consequences. As we will see in Lemma 3.9, the map $\alpha$ is null homotopic. Thus we will have proved Theorem I.4.8. If $\Pi_{1}$ and $\Pi_{2}$ are quasifibrations, and the fibers are homotopy equivalent by a null homotopic map, then the fibers are contractible and Theorem I.4.8 is a formal consequence.

Now recall the construction without differentials. Let us suppose this construction is for the birds (an eminently plausible hypothesis). Then Theorem I.4.8 is probably false for it. Thus either $\Pi_{1}$ or $\Pi_{2}$ is not a quasifibration. The writer happens to know that $\Pi_{1}$ is, and therefore $\Pi_{2}$ probably is not. This means that any proof that, for the construction with differentials, $\Pi_{2}$ is a quasifibration, will have to be subtle-it must 
distinguish Constructions I.4.6 and I.4.7.

Caution 1.6. As was already pointed out in Caution I.5.4, the homotopies of this article have a way of wandering outside their simplicial set. I know no general condition which guarantees that they will be well defined. On this point the reader would be ill advised to trust the author. It is very easy to make mistakes.

There is one special case of the prototype homotopy that deserves special mention. Consider the simplicial set

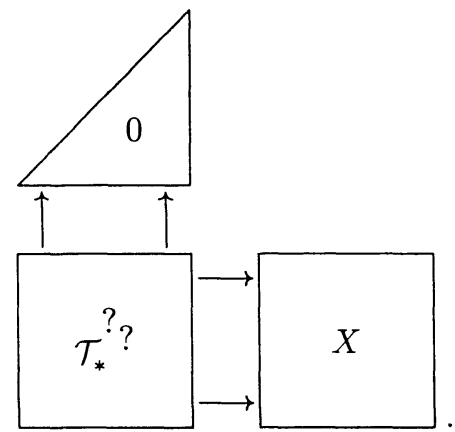

The homotopy

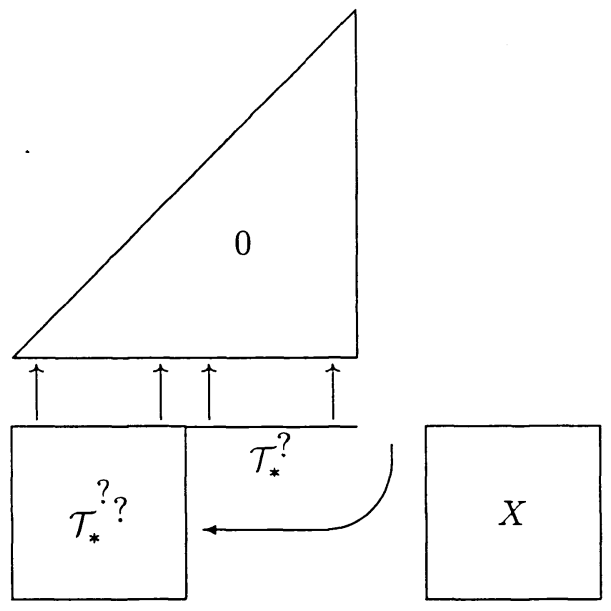

takes a simplex 


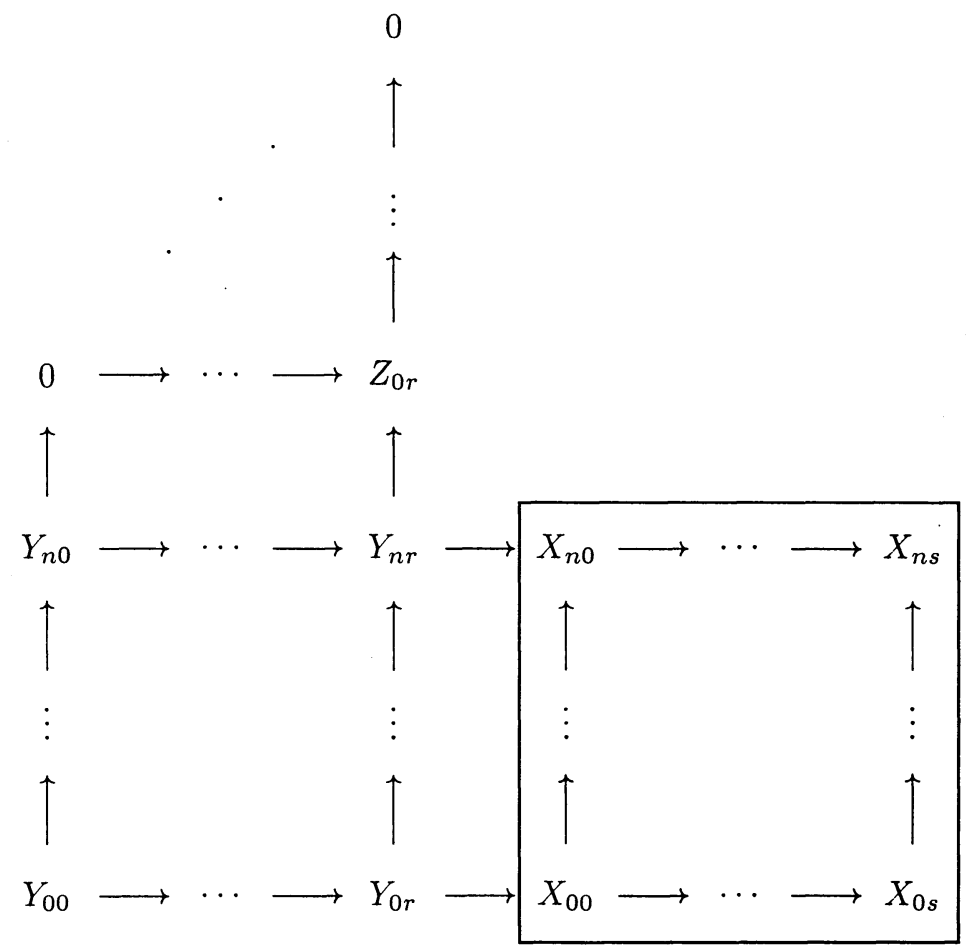

To simplices of the type 


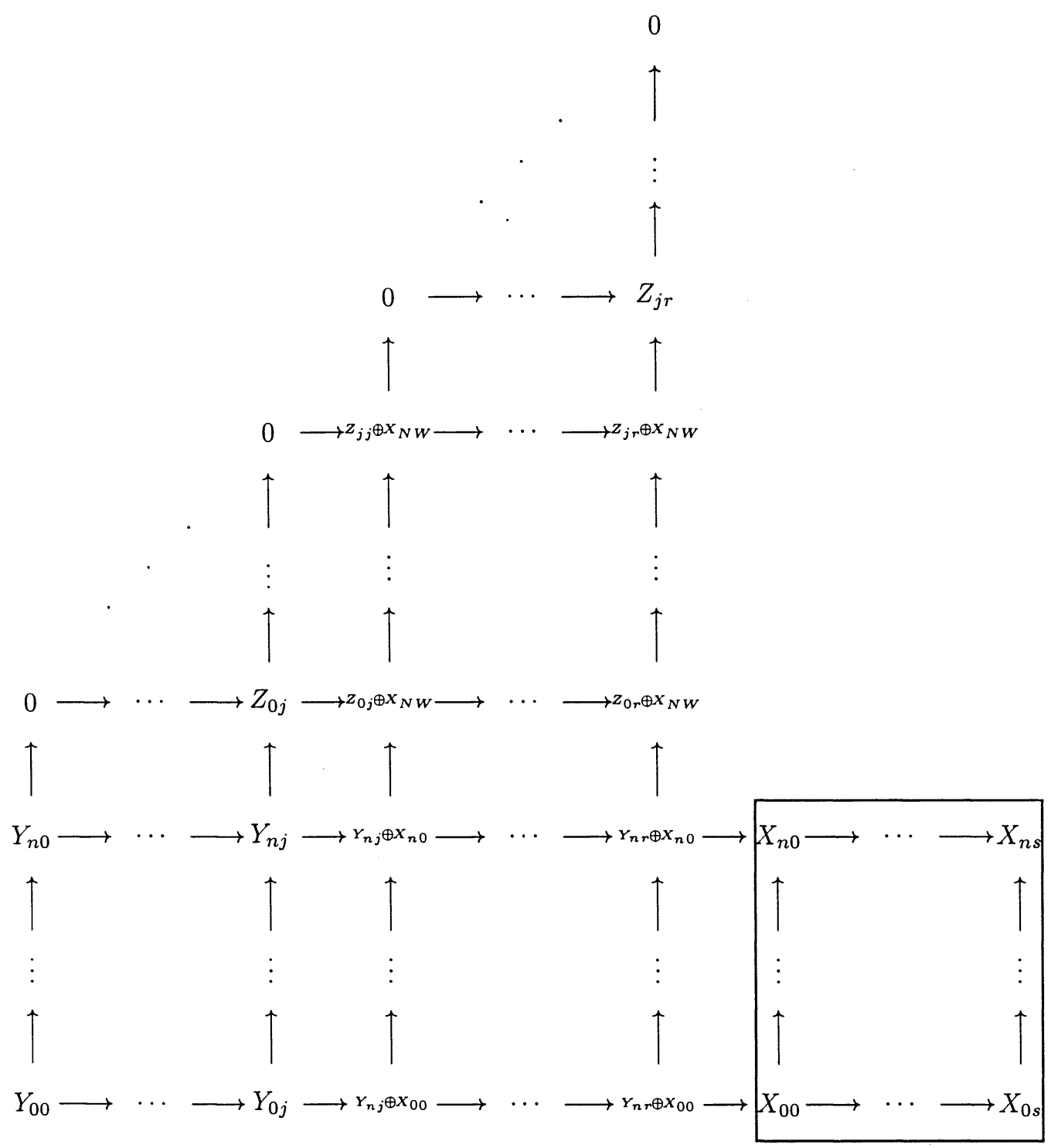

and the point I want to make is that this homotopy manages to combine the two homotopies of Prototype Quasifibration 1.2 into one. The horizontal and vertical parts of Prototype Quasifibration 1.2 can be seen in the single cell featured above. Precisely, in the highlighted rectangle 


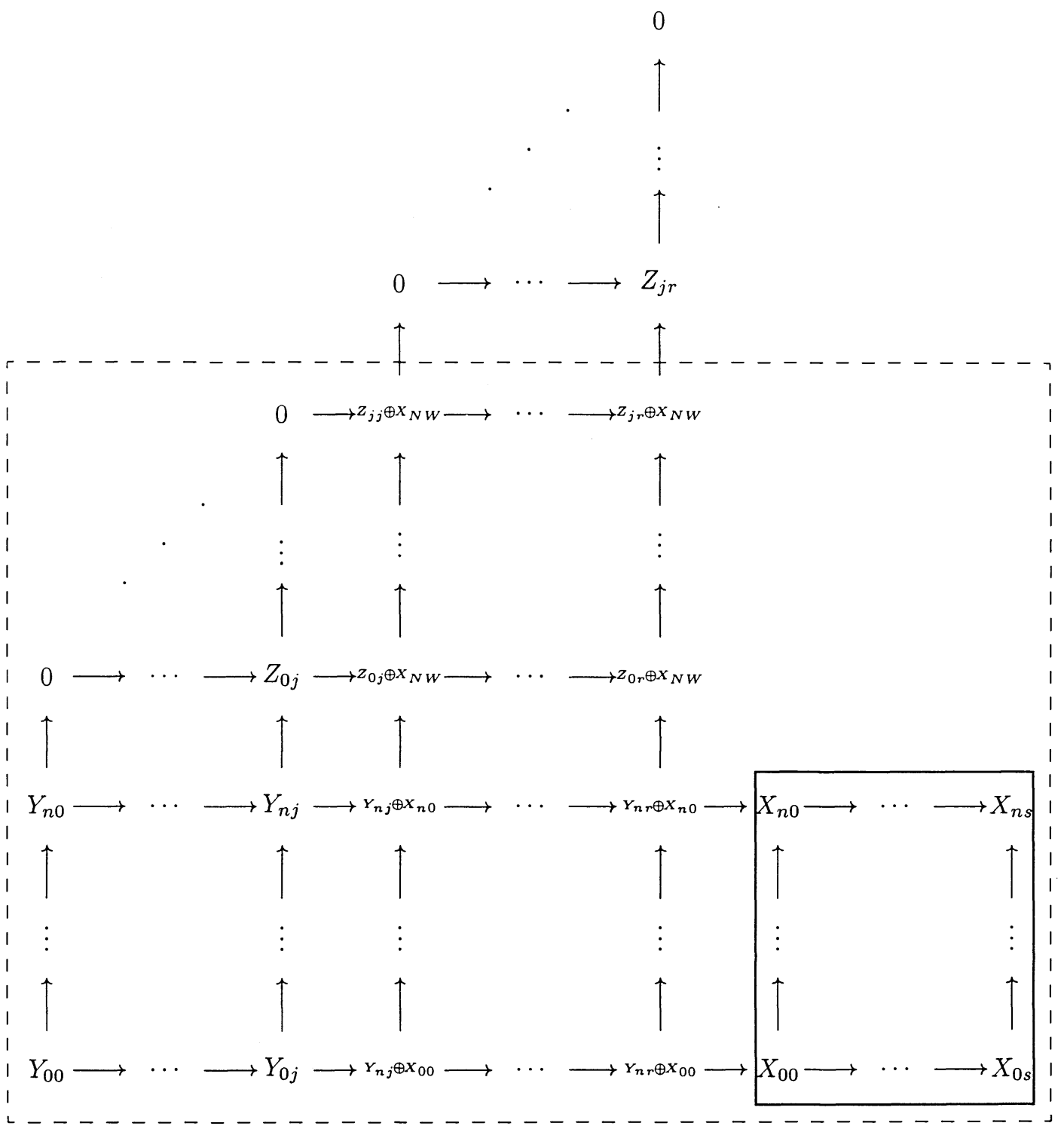

we recognize our first (horizontal) homotopy, and in 


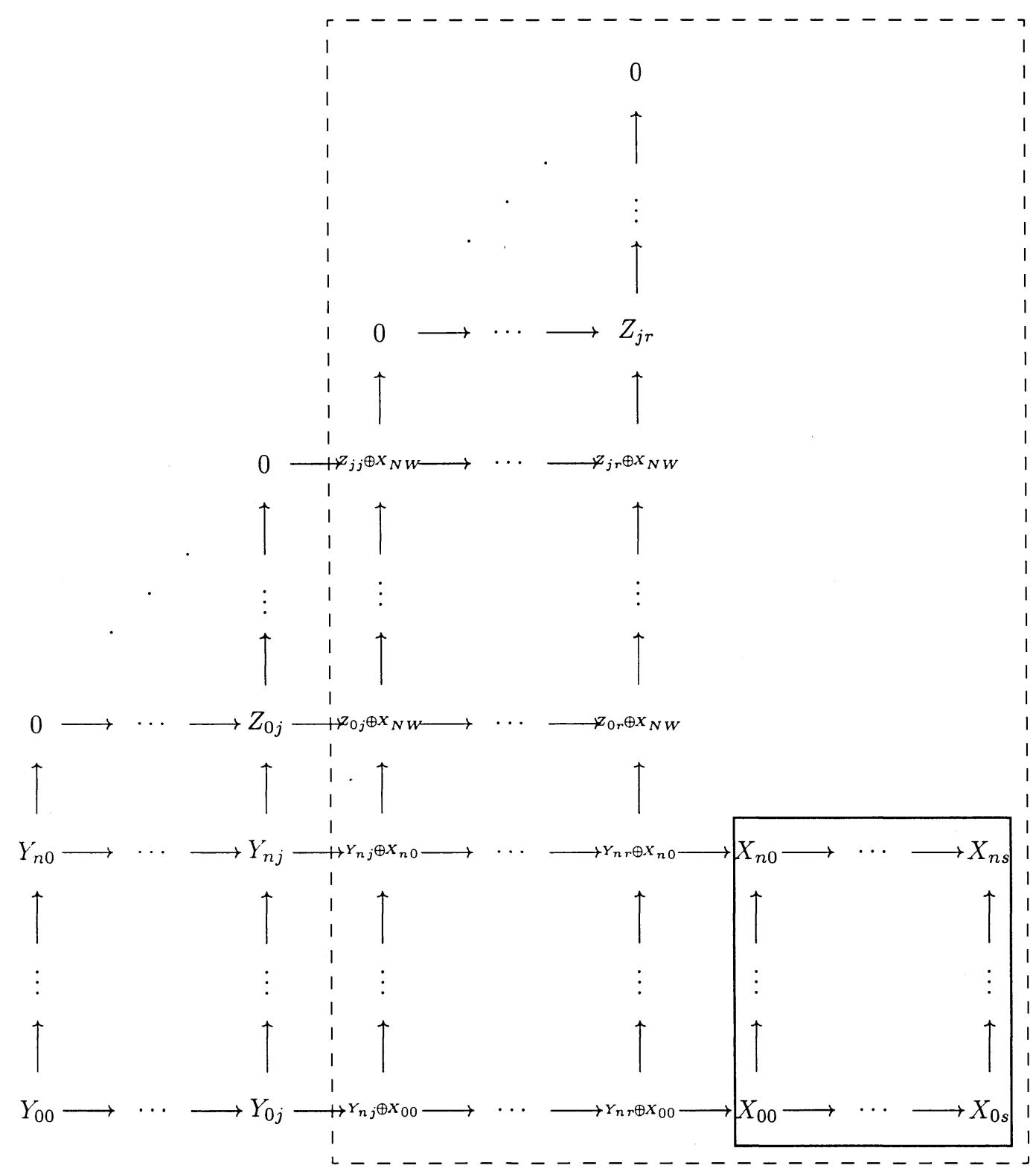

the second, (vertical) homotopy.

Thus, the map

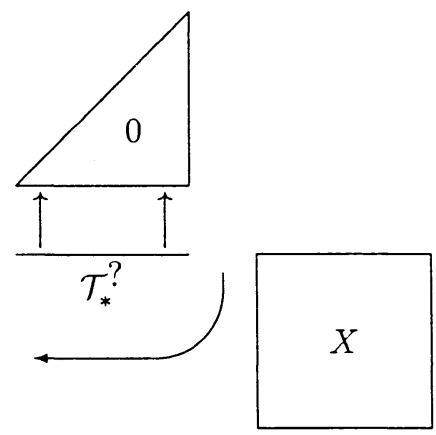


factors through

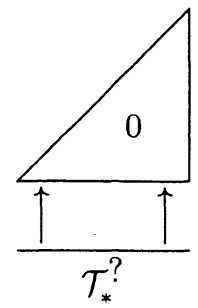

and is independent of $X_{N W}$. This is a fact we have often used. See Theorem I.5.1 and Lemma I.8.3.

We leave to the reader the formulation of the transpose and duals of all our constructions.

Only two homotopies, which have already come up in this series of articles, are not immediately covered by the prototypes we have discussed in the section so far. The homotopy in Lemma I.8.8 is spurious, as I already mentioned there. It wants to be a truncation, but it is a fake; such phenomena do arise in $\operatorname{Gr}(\mathcal{A})$. The other questionable homotopy is the one we denoted

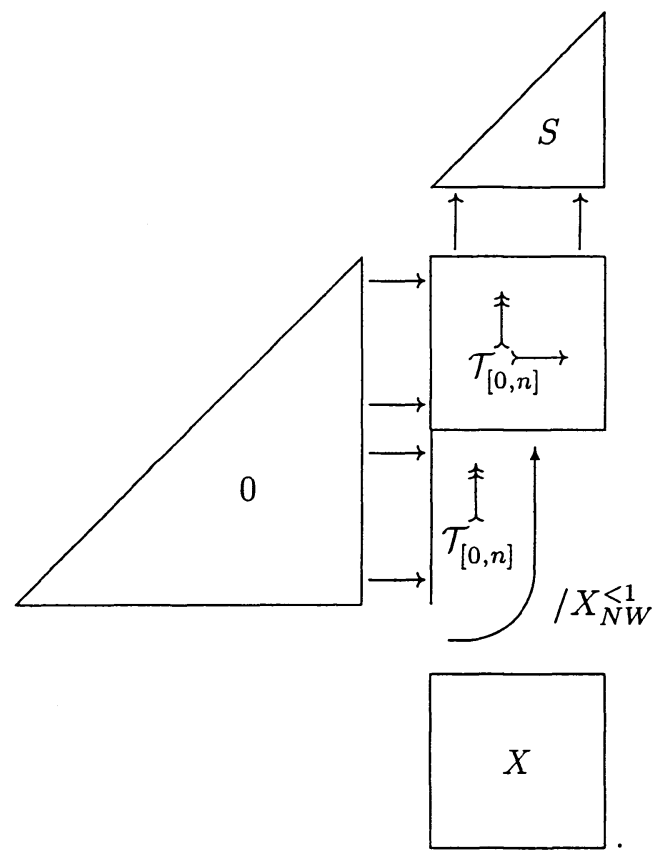

Although the homotopy is not explicitly on our list, it is a cross between the prototype homotopy and the truncation. Since we will have the occasion to use this homotopy again, we should reflect on it a little.

One is certainly allowed to compose homotopies, and to take diagonals in product homotopies. We leave it to the reader to amuse himself by showing how this homotopy can be recovered from our basic building blocks. If the reader does not find such things amusing, he can find a fairly detailed account in the proof of Lemma III.1.2.

But like all homotopies involving the truncation, it should be viewed with extreme suspicion. As was said in the beginning of the section, these homotopies often fail to contract. When we first studied the above hommotopy, in the proof of Lemma I.7.6, 
we said that it takes the identity to the map
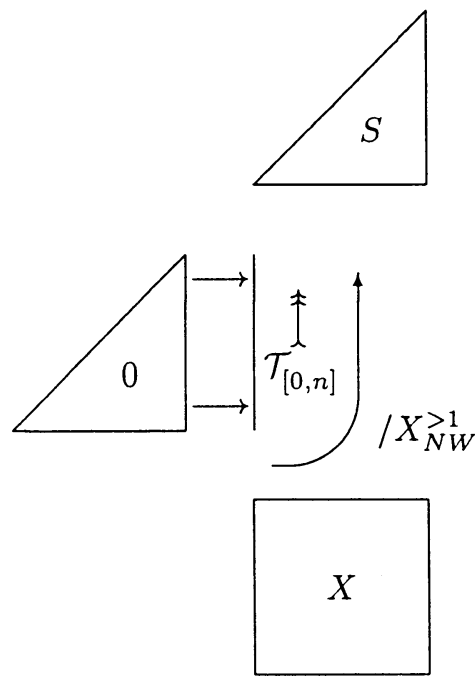

So far we have said nothing; this is a notational tautology. But our next statement was that this map factors through

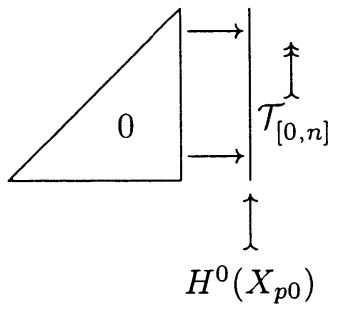

This is a non-trivial statement, and the reader should now learn to beware of these. It amounts to the assertion that certain differentials are uniquely determined by $X$, $S$ and

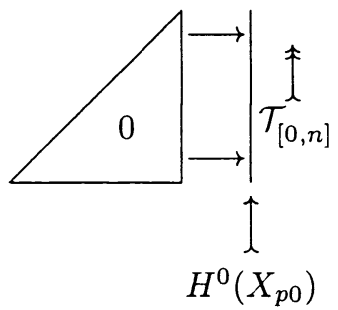

Since we have already given a discussion of the problems that arise from the truncation, we leave this point to the reader to ponder over. Without the $S$ the statement would definitely be false; exactly as with the previous example of a truncation, the space 


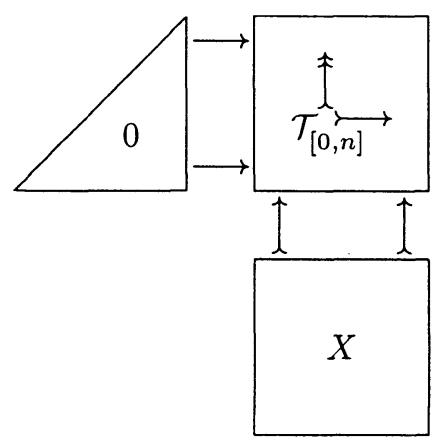

would not even be connected.

2. A Discussion of Construction I.4.6. Motivation. In this section, we will discuss in some detail what can be deduced about Construction I.4.6. Because the results in Section I.8 nearly all held for both the dumb Construction I.4.6 and the intelligent Construction I.4.7, it is curious to see just how much of Theorem I.4.8 remains valid for the "Construction without Differentials."

There is a commutative diagram whose rows are fibrations

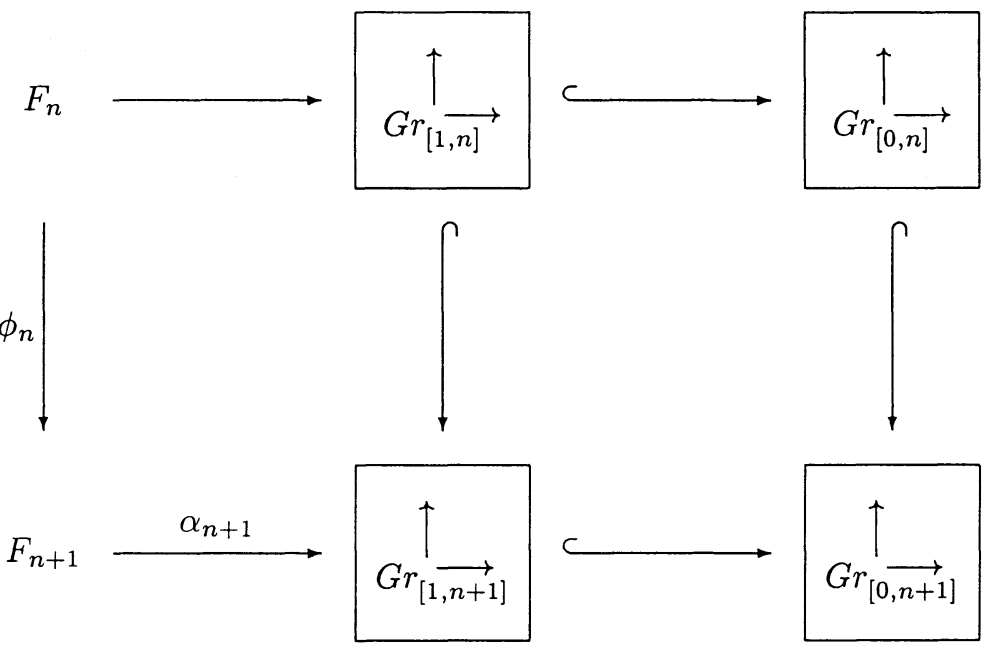

With the notable exception of Lemma I.8.10, everything in Section I.8 is valid for both constructions. In particular, $\phi_{n}$ is a homotopy equivalence. Furthermore, in this section we will show that $\alpha_{n+1} \circ \phi_{n}$ is null homotopic.

This is really all we know, but it is enough to deduce a surprising amount about the simplicial set

$$
\underset{[0, \overrightarrow{ } \underset{[0, n]}{\longrightarrow}}{\longrightarrow}
$$

In particular, this simplicial set decidedly is not a $K(\Pi, 1)$. It has interesting higher homotopy which is related to the higher $K$-theory of $\mathcal{A}$.

There are nevertheless formal reasons to suspect that the homotopy groups of 


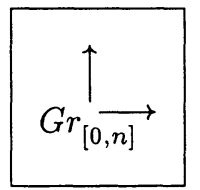

give a bad $K$-theory. Suppose we iterate the construction in the sense of Waldhausen, to define a spectrum of iterated $G r_{[0, n]}^{\longrightarrow}$ s. This makes sense; I leave the definition to the reader. In particular, this spectrum-valued $K$-theory satisfies an additivity theorem. The additivity theorem says the following. Suppose $\mathcal{A}$ and $\mathcal{B}$ are two abelian categories, and $F^{\prime}, F$, and $F^{\prime \prime}$ are functors $G r^{b}(\mathcal{A}) \rightarrow G r^{b}(\mathcal{B})$. Suppose there is an "exact" sequence of natural transformations

$$
0 \Rightarrow F^{\prime} \stackrel{\phi}{\Rightarrow} F \stackrel{\psi}{\Rightarrow} F^{\prime \prime} \Rightarrow 0 .
$$

That is, for every object $X$ in $G r^{b}(\mathcal{A})$, there is a sequence

$$
0 \rightarrow F^{\prime}(X) \stackrel{\phi_{x}}{\rightarrow} F(X) \stackrel{\psi_{x}}{\rightarrow} F^{\prime \prime}(X) \rightarrow 0
$$

which is exact in $G r^{b}(\mathcal{B})$ in the bizarre sense of Definition I.4.3. We remind the reader that this means exactness in the middle, but for $\operatorname{ker}\left(\phi_{x}\right)$ and $\operatorname{coker}\left(\psi_{x}\right)$ it means only that $\Sigma \operatorname{ker}\left(\phi_{x}\right) \simeq \operatorname{coker}\left(\psi_{x}\right)$, where " $\simeq$ " denotes that the two are equivalent up to finite filtrations. There is a finite filtration of $\Sigma \operatorname{ker}\left(\phi_{x}\right)$ and a finite filtration of $\operatorname{coker}\left(\psi_{x}\right)$, so that the associated graded objects are isomorphic.

Then the additivity theorem asserts that $K(F)=K\left(F^{\prime}\right)+K\left(F^{\prime \prime}\right)$, where $K$ is the functor obtained from iterating Construction I.4.6.

In particular, let $k$ be a field and let $\bar{k}$ be its separable closure. Let $\mathcal{A}=\mathcal{B}$ be the category of finite-dimensional vector spaces over $\bar{k}$. Choose some element $\sigma \in \operatorname{Gal}(\bar{k} / k)$, an automorphism of $\bar{k}$. Define $F^{\prime}, F$, and $F^{\prime \prime}: \mathcal{A} \rightarrow \mathcal{B}$ as follows:

(1) $F^{\prime}=\Sigma^{-1}$ is the desuspension;

(2) $F=0$

(3) $F^{\prime \prime}=\sigma$ is induced by $\sigma: \bar{k} \rightarrow \bar{k}$.

Then clearly there is a short exact sequence

$$
0 \Rightarrow F^{\prime} \Rightarrow F \Rightarrow F^{\prime \prime} \Rightarrow 0
$$

and we deduce

$$
0=K(F)=K\left(F^{\prime}\right)+K\left(F^{\prime \prime}\right)=K\left(\Sigma^{-1}\right)+K(\sigma) .
$$

It follows that $K(\sigma)=-K\left(\Sigma^{-1}\right)$ is independent of $\sigma \in \operatorname{Gal}(\bar{k} / k)$. In particular, $K(\sigma)=K(1)=1$, so the Galois group acts trivially on $K\left(G r^{b}(\mathcal{A})\right)$.

This means that $K\left(G r^{b}(\mathcal{A})\right)$, the iterated version of Construction I.4.6, is really very far removed from $K(\mathcal{A})$. It is well known that $K_{1}(\bar{k})=\bar{k}^{*}$, with the natural (non-trivial) Galois action.

Section 2 is mostly intended to serve as a warning of potential pitfalls in the theory. 


\section{End of Motivation}

In Section I.8 we proved Theorem I.4.8. This means that we know the homotopy type of Construction I.4.7. However, all but the last step of the proof was equally valid for Construction I.4.6. In this section we will analyze the consequences that follow.

We begin by recalling that, in Lemma I.7.15, we proved that the homotopy fiber of the inclusion $G r_{[1, n]} \leftrightarrow \operatorname{rr}_{[0, n]}$ is the simplicial set

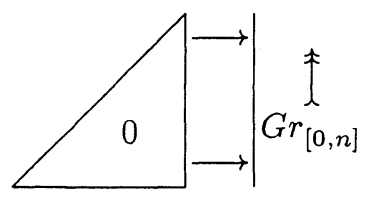

In Lemma I.8.8 we proved, modulo the earlier steps, that the natural map

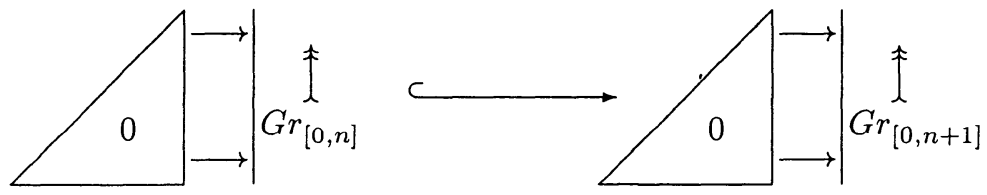

is a homotopy equivalence for all $n \geq 1$. The reader can check that the inclusion map above is the natural map of the fibers; thus in the diagram where the row are fibrations and the inclusions are obvious:

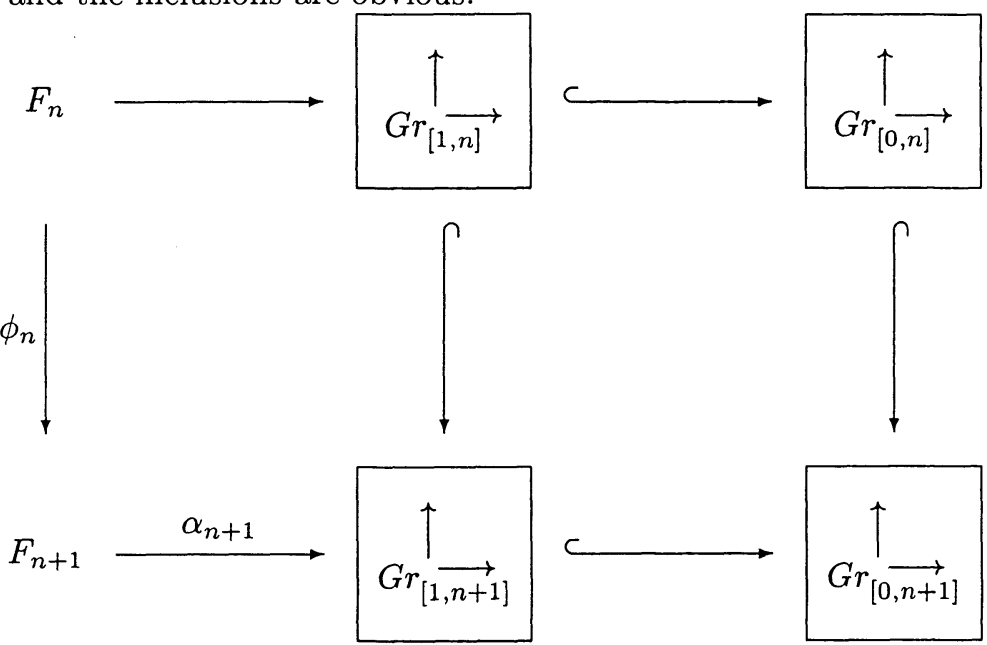

we have proved that $\phi_{n}$ is a homotopy equivalence.

What turns out to be quite easy to show is

LEMMA 2.1. In the above diagram, if $n=1$, then the composite

$$
F_{1} \stackrel{\phi_{1}}{\longrightarrow} F_{2} \stackrel{\alpha_{2}}{\longrightarrow} \underset{G r_{[1,2]}}{\uparrow}
$$

is null homotopic.

Proof. For this proof, it is convenient to use the model of $F_{1}$ given in Lemma I.8.7. 


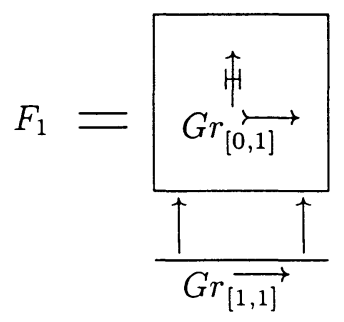

Then the composite map $\alpha_{2} \circ \phi_{1}$ is given by

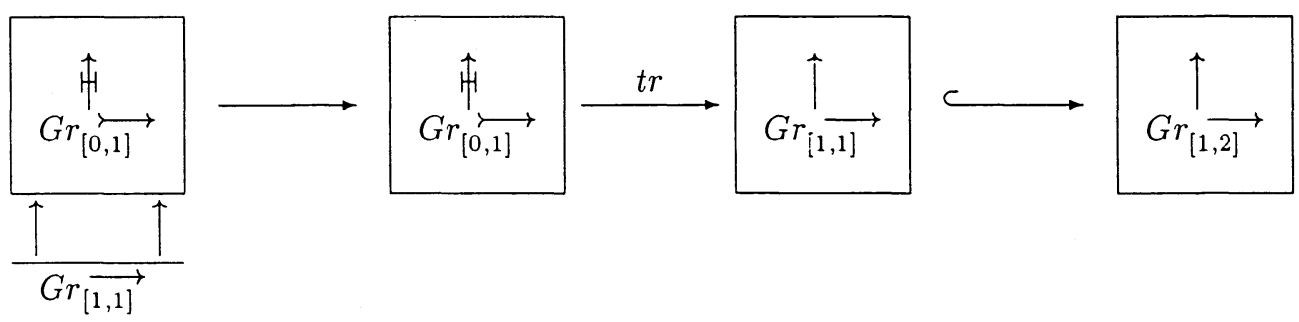

where $t r$ stands for the truncation map. The key point in the proof is that the following diagram commutes up to sign

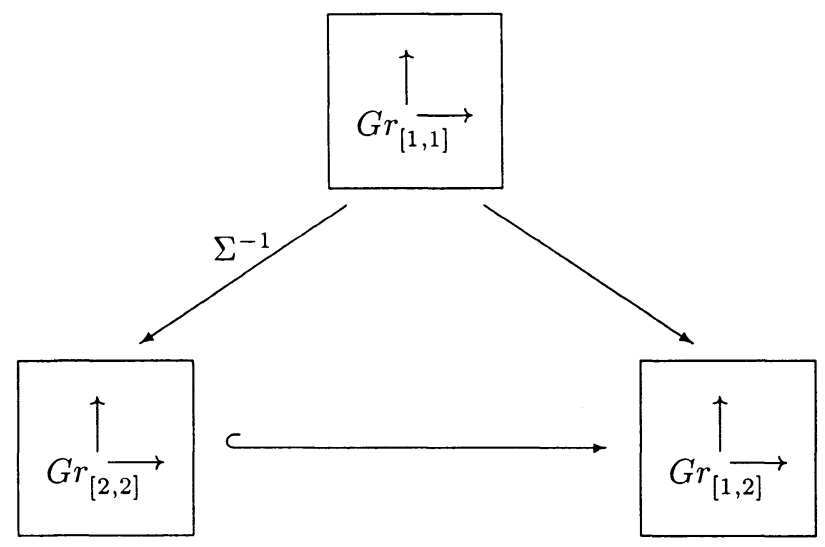

In other words, suspension induces the map -1 , at least on $G r_{[1,1]}$.

Let us accept this fact temporarily, and show how to complete the proof of the lemma. It suffices then to show that the composite

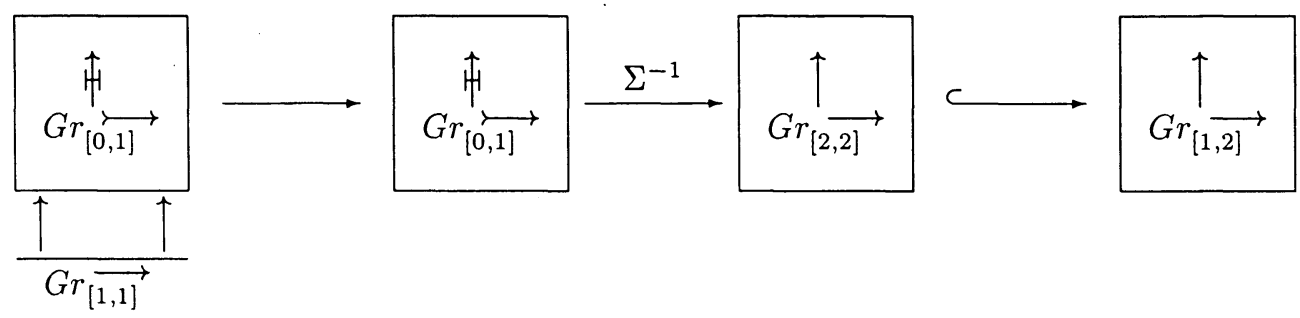

is null homotopic; but this map is homotopic to 


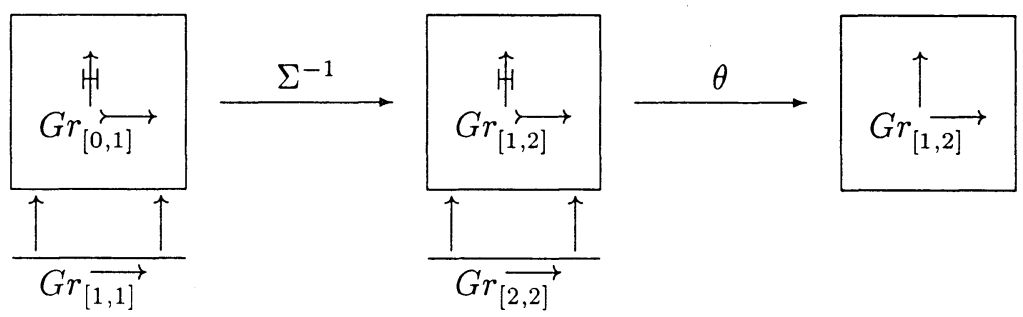

and $\theta$ is clearly null homotopic.

Now we must return to the proof of the fact that, on $G r \underset{[1,1]}{\longrightarrow}$, the map $\Sigma$ is homotopic to -1 . We need to show that the composite
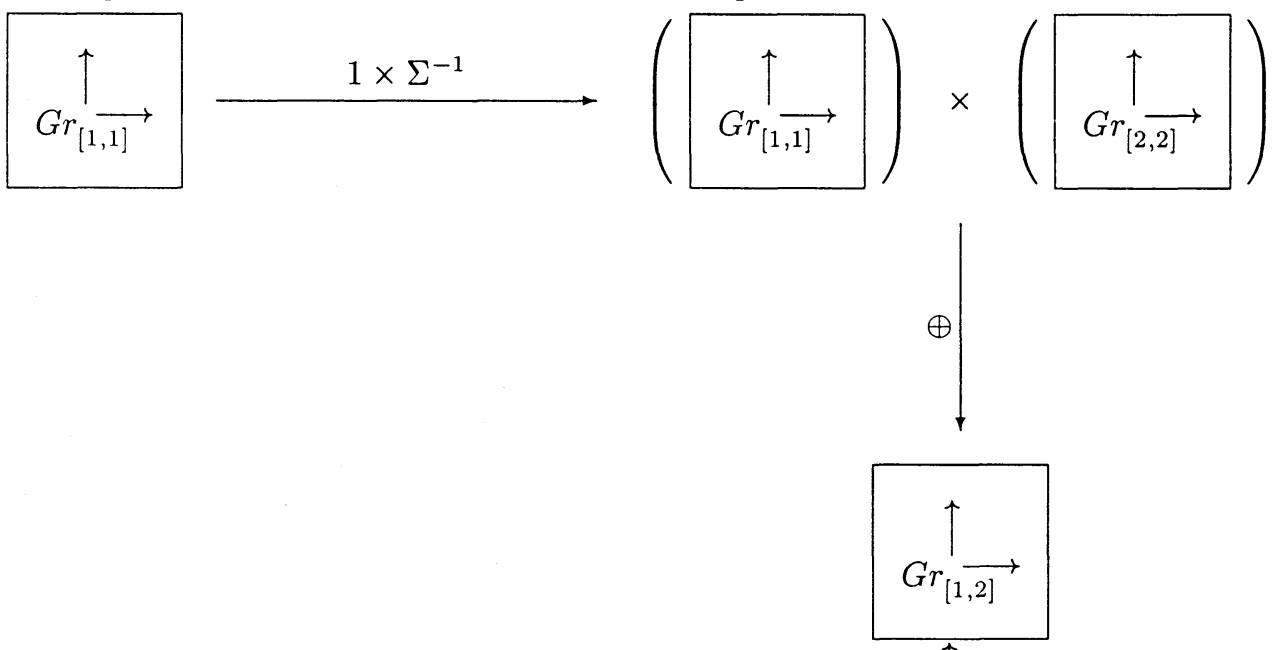

is null homotopic. What makes this easy is the fact that $G r_{[1,1]}$ is just Quillen's $Q$-construction for the abelian category $\mathcal{A}$. Let $\mathbf{1}$ be the category

$$
1=\rightarrow
$$

Then $\operatorname{Hom}(\mathbf{1}, \mathcal{A})$ is an abelian category, and it is well known that the projection $\operatorname{Hom}(\mathbf{1}, \mathcal{A}) \rightarrow \mathcal{A}^{2}$, which forgets the morphism, induces a homotopy equivalence on the $Q$-constructions.

There are two functors $\mathcal{A} \rightarrow \operatorname{Hom}(\mathbf{1}, \mathcal{A})$

(1) $F_{1}$ is the functor $F_{1}(a)=a \stackrel{0}{\rightarrow} a$;

(2) $F_{2}$ is the functor $F_{2}(a)=a \stackrel{1}{\rightarrow} a$.

Because the composites

$$
\mathcal{A} \rightrightarrows \operatorname{Hom}(1, \mathcal{A}) \rightarrow \mathcal{A}^{2}
$$

are equal, it follows that $F_{1}$ and $F_{2}$ are homotopic. Now observe that there is a map

$$
\phi: H o m(\mathbf{1}, \mathcal{A}) \rightarrow \operatorname{Gr}(\mathcal{A})
$$


where

$$
\phi(a \stackrel{f}{\longrightarrow} b)=\left\{\begin{aligned}
k e r(f) & \text { in dimension } 1 \\
\operatorname{coker}(f) & \text { in dimension } 2
\end{aligned}\right.
$$

and this is clearly a functor of bicategories; it induces a map on $Q$-construction. Now $F_{1} \simeq F_{2}$ implies $\phi \circ F_{1} \simeq \phi \circ F_{2}$, but $\phi \circ F_{2}=0$ while $\phi \circ F_{1}=1+\Sigma^{-1}$, the map we are studying.

Corollary 2.2. The space $F_{1}$ is also the homotopy fiber of the map

$$
G r \underset{[-n,-1]}{\longrightarrow}
$$
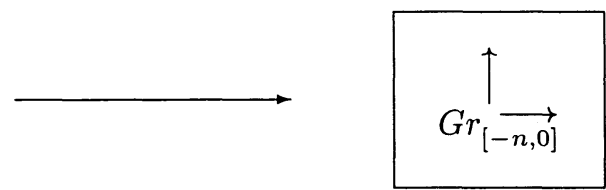

Proof. Recall that $F_{1}$ was defined to be the homotopy fiber of the map

$$
G \underset{[1,1]}{\uparrow}
$$

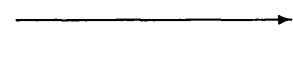

$$
G r_{[0,1]}
$$

and that our key lemma, Lemma I.8.8, shows that this is homotopy equivalent to the fiber of

$$
G \underset{[1, n]}{\longrightarrow}
$$
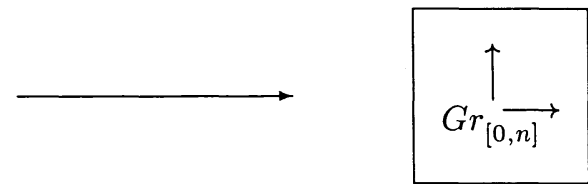

In the proof of Lemma 2.1 , we showed that the diagram

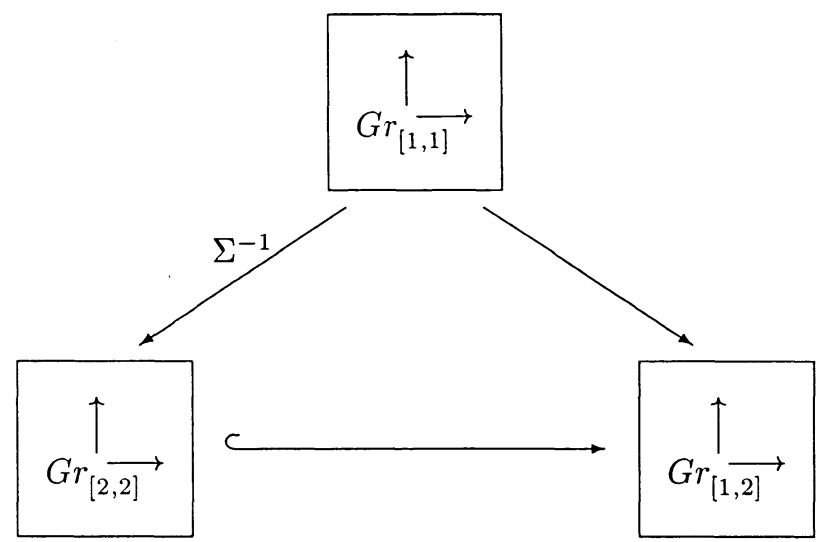

commutes up to sign. On the other hand, $\Sigma$ is an isomorphism. It follows that the fibers of the two maps
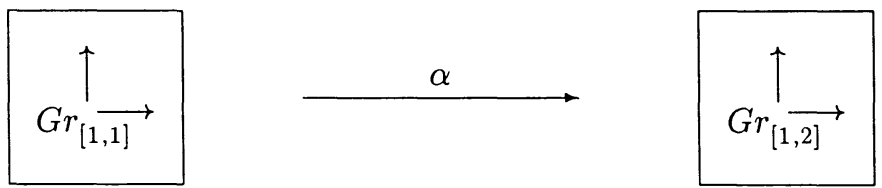


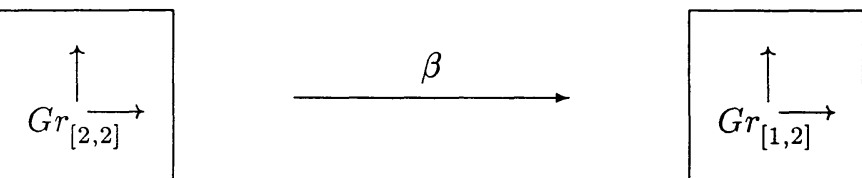

are, up to an isomorphism, the same. The fiber of $\beta$ is, up to suspension, the space $F_{1}$. Hence $F_{1}$ is also the homotopy fiber of $\alpha$. By the dual of Lemma I.8.8, the homotopy fiber of $\alpha$ agrees with the homotopy fiber of the map
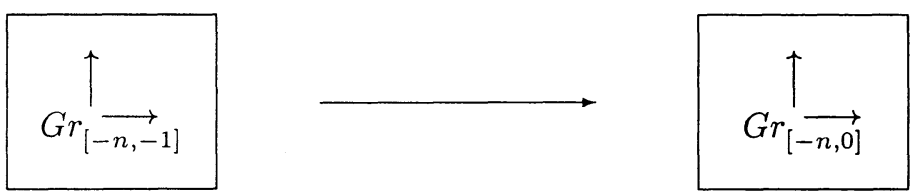

Corollary 2.3. The composite

$$
F_{1} \longrightarrow F_{n} \stackrel{\alpha_{n}}{\longrightarrow} \underset{[0, n]}{\longrightarrow}
$$

is null homotopic, for $n \geq 2$.

Proof. In the commutative diagram

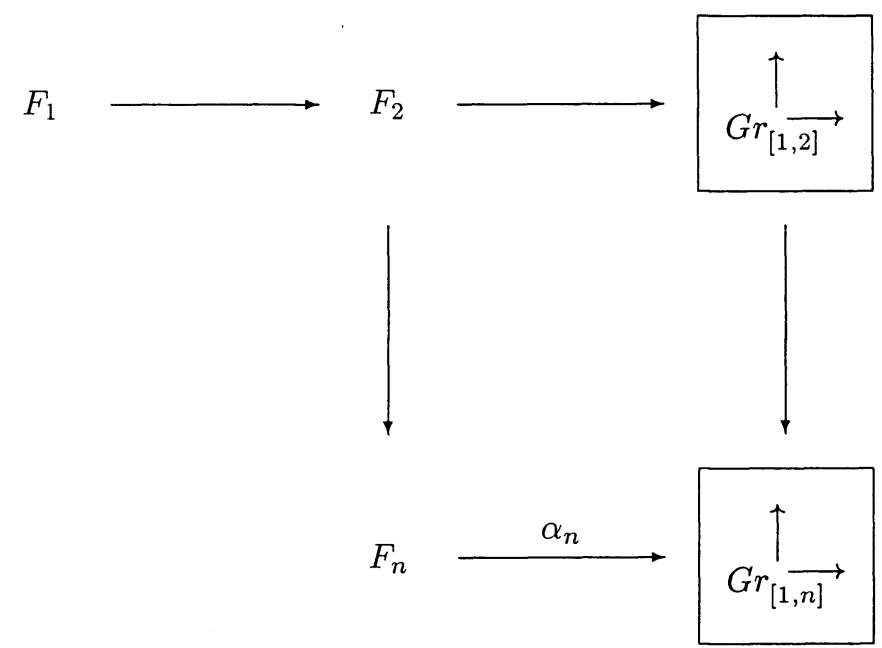

the top row vanishes, by Lemma 2.1. But then the commutativity implies the vanishing of 

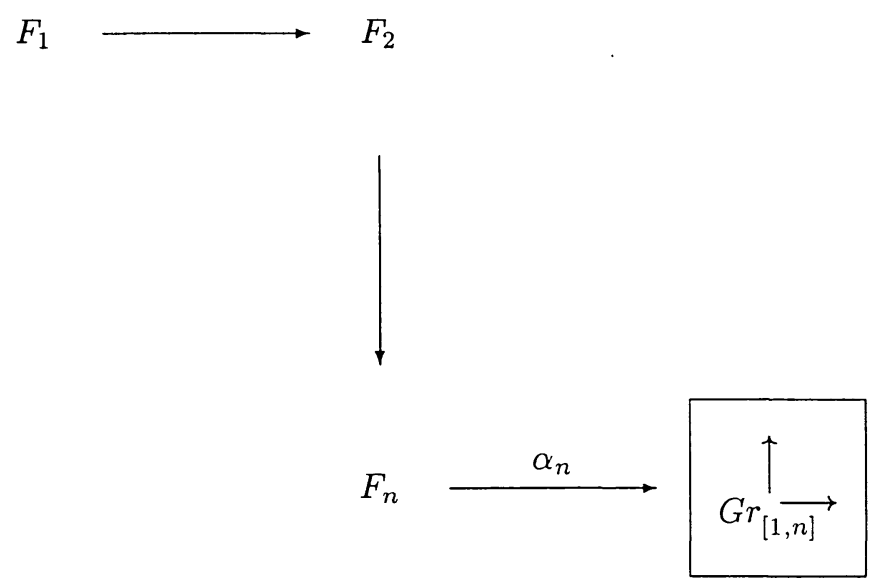

COROllary 2.4. The map $F_{n} \longrightarrow \quad G r \underset{[1, n]}{\longrightarrow} \quad$ is null homotopic whenever $n \geq 2$.

Proof. By Corollary 2.3, the composite

$$
F_{1} \longrightarrow F_{n} \stackrel{\alpha_{n}}{\longrightarrow} \underset{\left[r_{[0, n]}\right.}{\uparrow}
$$

vanishes. By Lemma I.8.8, the map $F_{1} \rightarrow F_{n}$ is a homotopy equivalence. Hence

$$
F_{n} \rightarrow \underset{[1, n]}{\uparrow}
$$

must be the null map.

Corollary 2.5. Given integers $m^{\prime} \leq m \leq n \leq n^{\prime}$. then the inclusion $\underset{n \geq m+\underset{[m, n]}{\longrightarrow} \hookrightarrow}{\uparrow} G r_{\left[m^{\prime}, n^{\prime}\right]}^{\longrightarrow}$ induces an injective map on homotopy groups, provided Proof. Applying Corollary 2.4 to the long exact sequences of the fibration

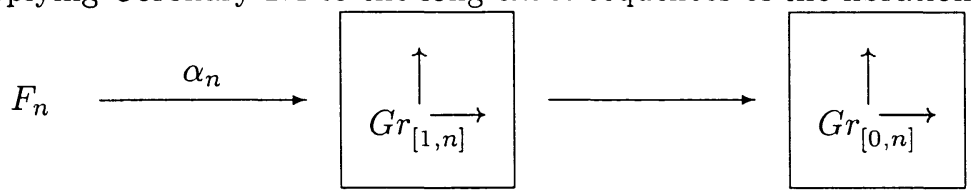

we have the result for $2 \leq n=n^{\prime}, m=1, m^{\prime}=0$. But the general case follows immediately from this case and its dual.

Thus, at the level of homotopy groups, the image of $\mathcal{A} \longrightarrow=G r_{[0,0]}$ in 
$\left(G r^{b}\right) \longrightarrow$ is simply its image in $G r_{[0,1]}$. Beyond that, the inclusions induce injections. But we can do even better than that.

Proposition 2.6. The image of the map

$$
\Pi_{i}\left(G r_{[0,0]}^{\uparrow}\right) \rightarrow \Pi_{i}\left(\left(G r^{b}\right)_{[0,0]}^{\uparrow}\right)
$$

is precisely the kernel of $1+\Sigma$ acting on $\Pi_{i}\left(G r^{b}\right)$, where $\Sigma$ is the suspension automorphism.

Proof. Recall Lemma I.8.8; it proves that in the diagram of fibrations

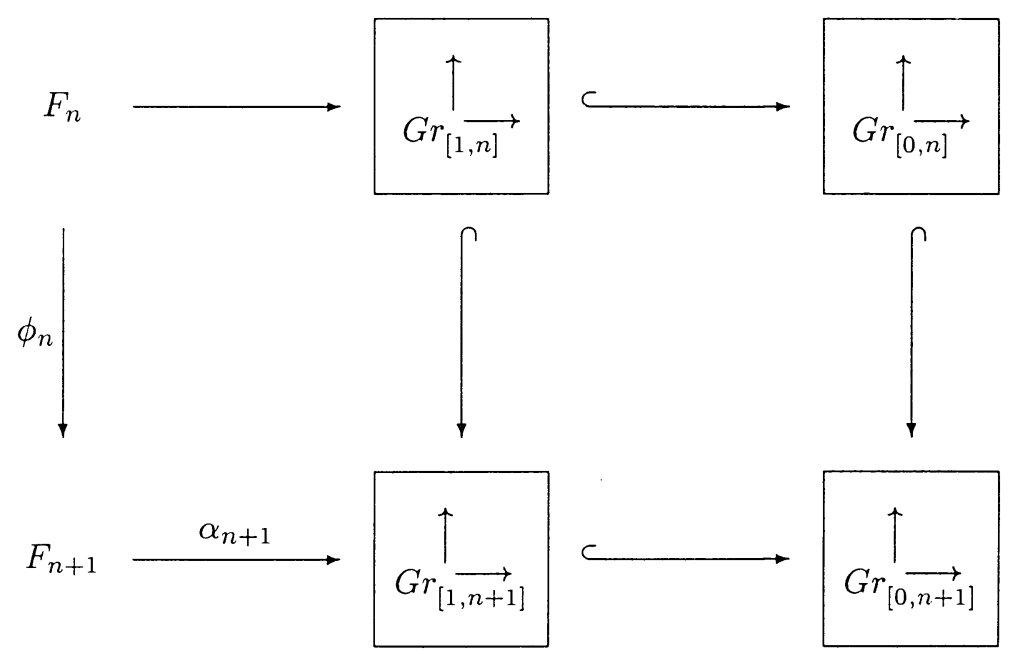

the $\operatorname{map} \phi_{n}$ is a homotopy equivalence. However, there is another natural diagram to consider: 


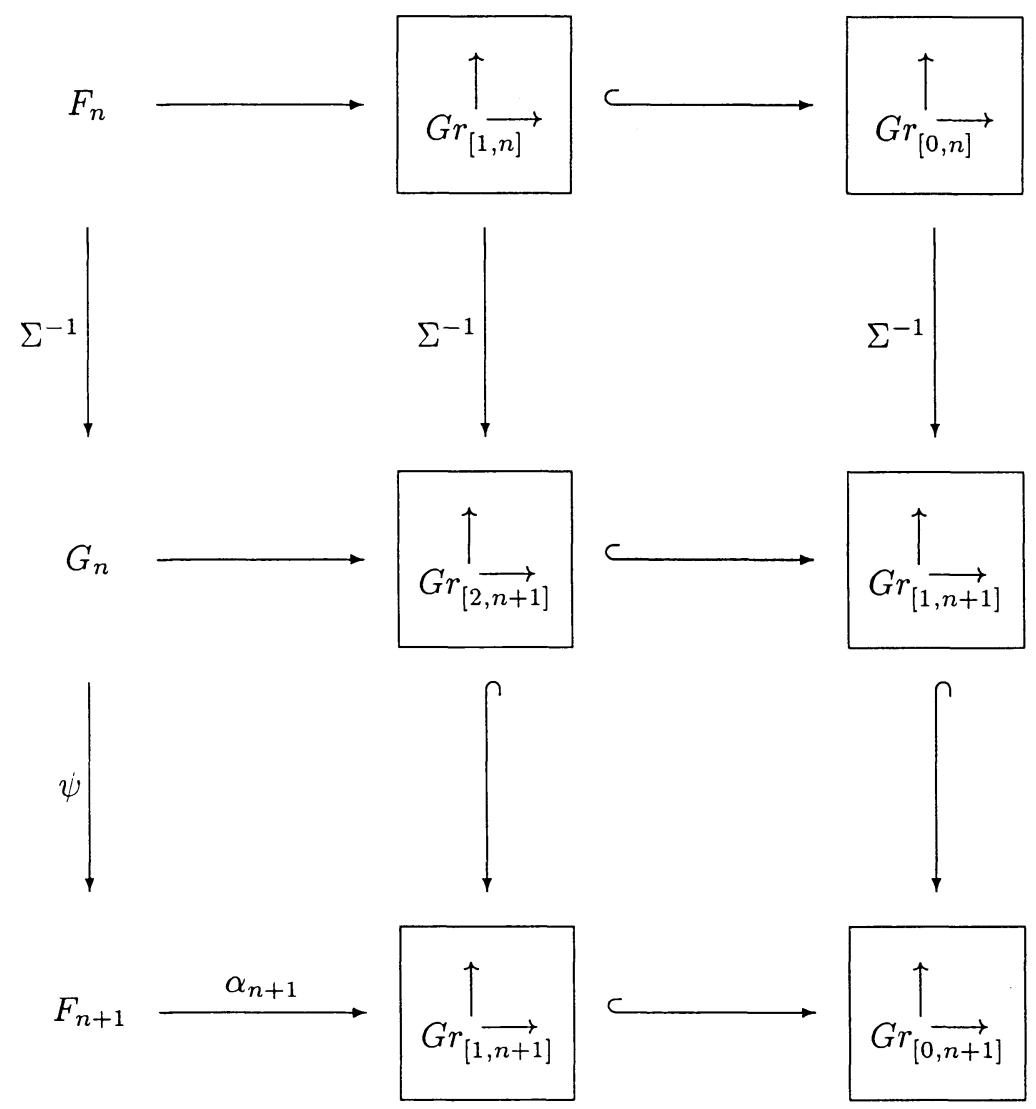

Now $\psi \circ \Sigma^{-1}$ is concretely given as the natural composite

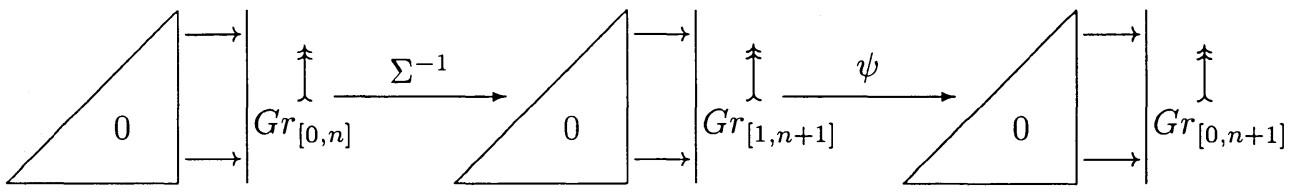

and this composite factors through

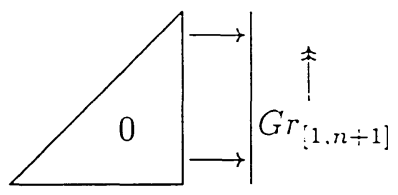

because any map in $G r_{[1, n+1]}$ is mono, when viewed as a morphism in $G r_{[0, n+1]}$. (Note that here we do not need the trick of replacing kernels by cokernels, as in the proof of Lemma I.8.10). This simplicial set that the map factors through is contractible. Therefore $\psi \circ \Sigma^{-1}$ is null homotopic. Hence $\phi+\psi \circ \Sigma^{-1}$ is a homotopy equivalence, because $\phi$ is.

We deduce a map of fibrations 


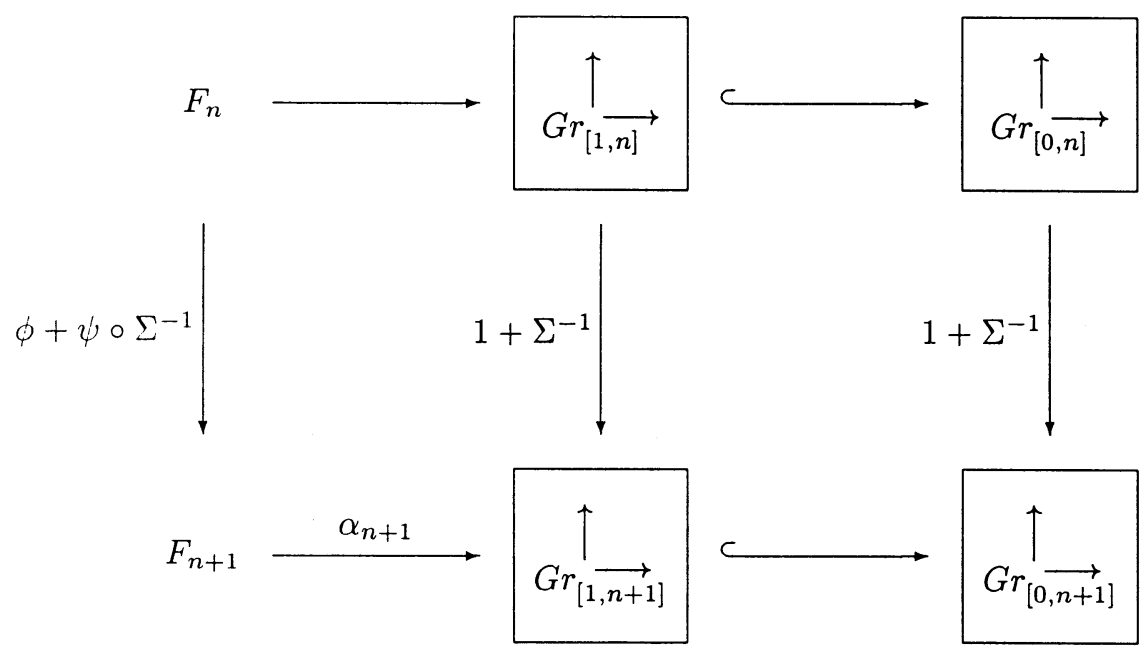

and the long exact sequence in homotopy in yields a diagram with exact rows and columns:

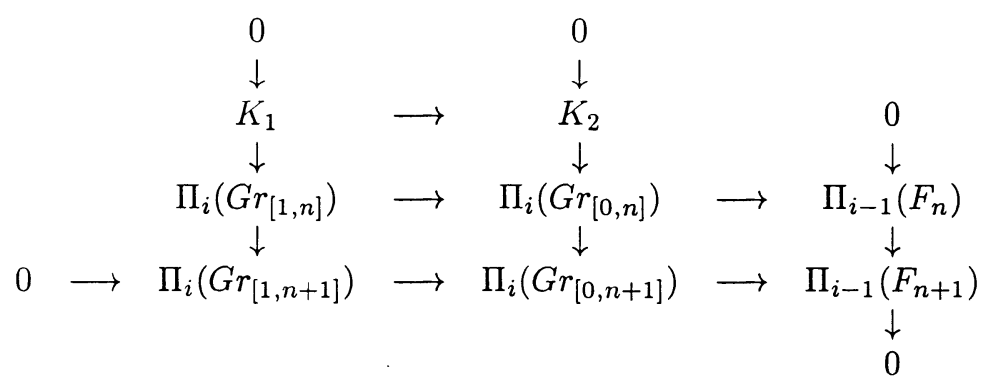

All the vertical maps are $1+\Sigma^{-1}$. The fact that the map in the bottom left hand corner is injective is Corollary 2.5. The fact the vertical map on the right is an isomorphism is the computation at the beginning of this proof.

Now an easy diagram chase establishes that $K_{1} \rightarrow K_{2}$ is surjective. For $n=1$, $K_{1}=\Pi_{i}\left(G r_{[1,1]}\right)$; therefore, for any $n, \operatorname{Ker}\left(1+\Sigma^{-1}\right)$ is the image of $\Pi_{i}\left(G r_{[1,1]}\right)$. The formula $1+\Sigma=\Sigma\left(1+\Sigma^{-1}\right)$ establishes the proposition; $1+\Sigma$ and $1+\Sigma^{-1}$ differ by an automorphism, hence have the same kernel.

Conclusions.

2.6.1. The image of the inclusion $\mathcal{A}=G r_{[0,0]} \rightarrow G r^{b}$, at the level of homotopy groups, is the kernel of $1+\Sigma$.

2.6.2. The homotopy groups of $G r^{b}$ are quite large; $\Pi_{i}\left(G r^{b}\right)$ is an extension of $\Pi_{i}\left(G r_{[0,1]}\right)$ by infinitely many copies of $\Pi_{i-1}\left(F_{1}\right)$. Thus, if there is a kernel to $\Pi_{i}\left(G r_{[0,0]}\right) \rightarrow \Pi_{i}\left(G r_{[0,1]}\right)$, then $\Pi_{i+1}\left(G r^{b}\right)$ will contain many copies of that kernel. In particular, $G r^{b}(\mathcal{A})$ is most definitely not a $K(\Pi, 1)$ (at least not for a general $\mathcal{A}$ ).

Problem 2.7. Does the inclusion $\mathcal{A} \rightarrow G r^{b}(\mathcal{A})$ induce a monomorphism in homotopy?

Problem 2.8. Does $1+\Sigma$ vanish on $G r^{b}(\mathcal{A})$ ?

REMARK 2.9. If $1+\Sigma$ vanishes, then $\mathcal{A} \rightarrow G r^{b}(\mathcal{A})$ is a homotopy equivalence. This is because the surjective map

$$
\operatorname{Ker}(1+\Sigma)=\operatorname{Im}\left(\Pi_{i}\left(G r_{[0,0]}\right)\right) \longrightarrow \Pi_{i}\left(G r^{b}\right)
$$

factors through $\Pi_{i}\left(G r_{[0, n]}\right)$ for all $n \geq 1$. It follows that $\Pi_{i}\left(G r_{[0, n]}(\mathcal{A})\right) \rightarrow \Pi_{i}\left(G r^{b}(\mathcal{A})\right)$ is surjective, but we also know from Corollary 2.5 that it must be injective. Hence it 
is an isomorphism. Thus $\Pi_{i}\left(G r_{[0,1]}\right) \cong \Pi_{i}\left(G r_{[0,2]}\right)$, hence $F_{2}$ is contractible, and so are all the $F_{n}$ 's.

3. Second Proof of Theorem I.4.8, and a Proof of Theorem I.7.1 (special case). Motivation. Section 3 will concern itself with the proof of Theorem I.7.1. As in the previous sections, the actual proof comes about as a badly tortured version of a fairly simple idea. We remind the reader that in Section I.7 we found a model for the homotopy fiber of

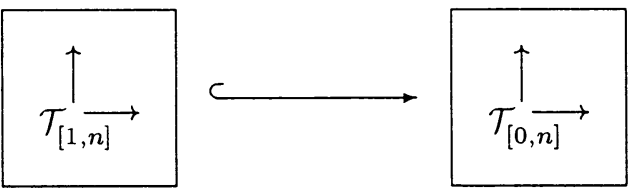

namely the simplicial set

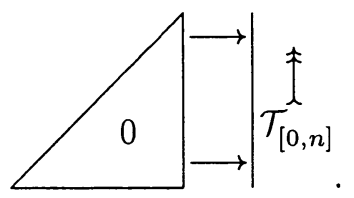

[Technically, I just lied; but it certainly suffices to prove the contractibility of

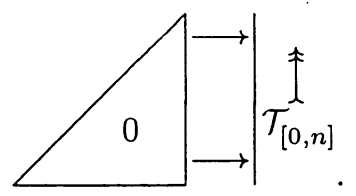

And morally, although we could not quite prove
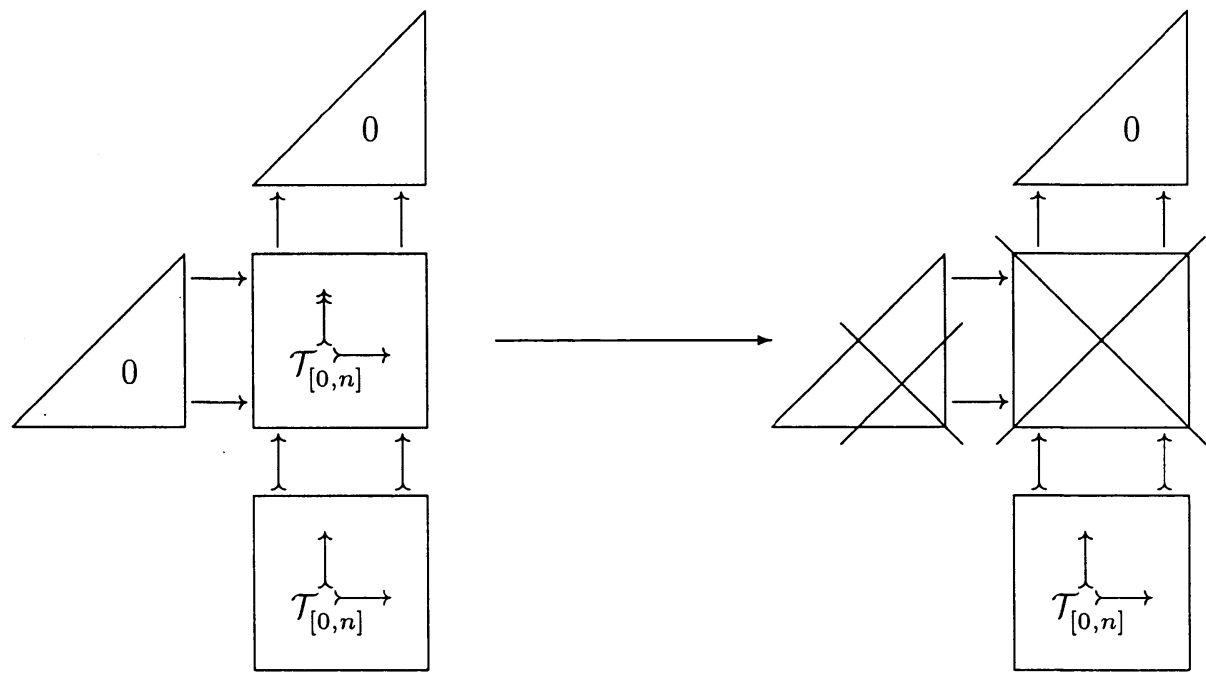

a quasifibration, it very nearly is. Anyway, white lies are permitted in the Motivation.]

In Section I.8, where we used this to prove Theorem I.4.8, it turned out to be useful to consider another model for this homotopy fiber, the simplicial set 


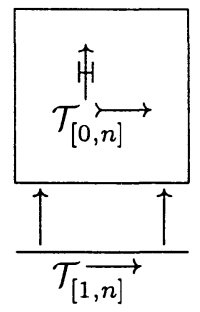

The idea that this should be a model for the homotopy fiber comes from the observation that
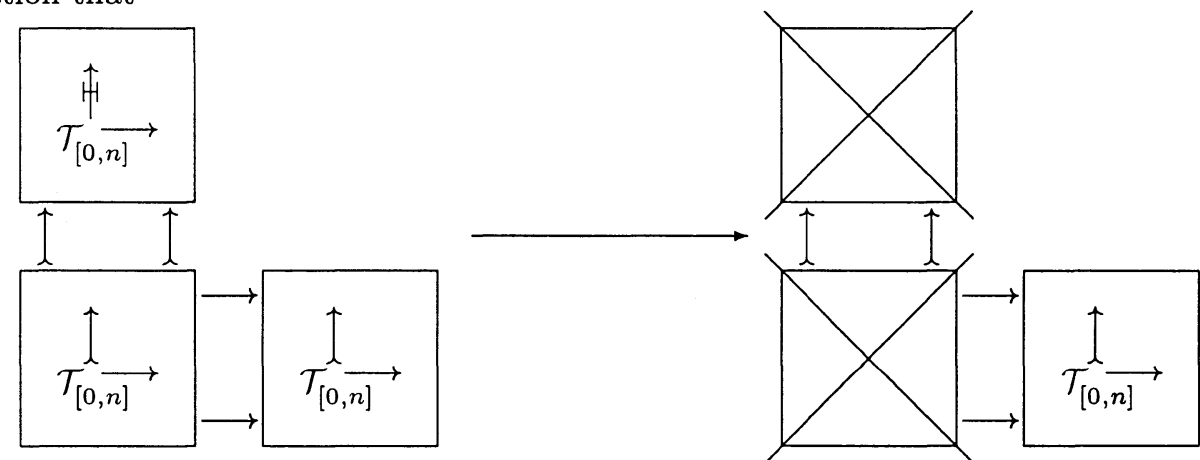

is probably a quasifibration. Although the writer has been unable to prove directly that it is, by a fairly round-about argument one can establish the existence of a homotopy equivalence
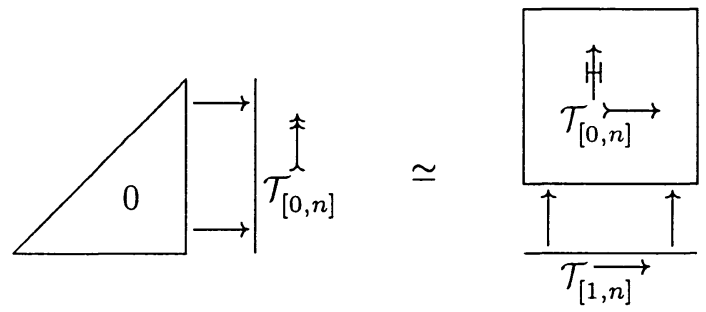

This means that the fiber over zero is the homotopy fiber. Not surprisingly, the identification of the fiber over 0 with the homotopy fiber is very natural in $n$. Theorem I.4.8 was almost an immediate consequence.

The idea of this section is to repeat the argument with the kernels chosen. In other words, we look at the simplicial set

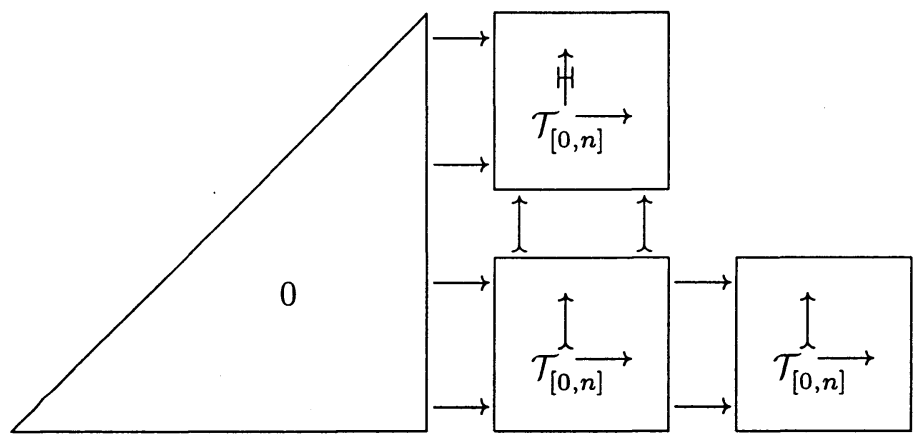

which I often prefer to denote 


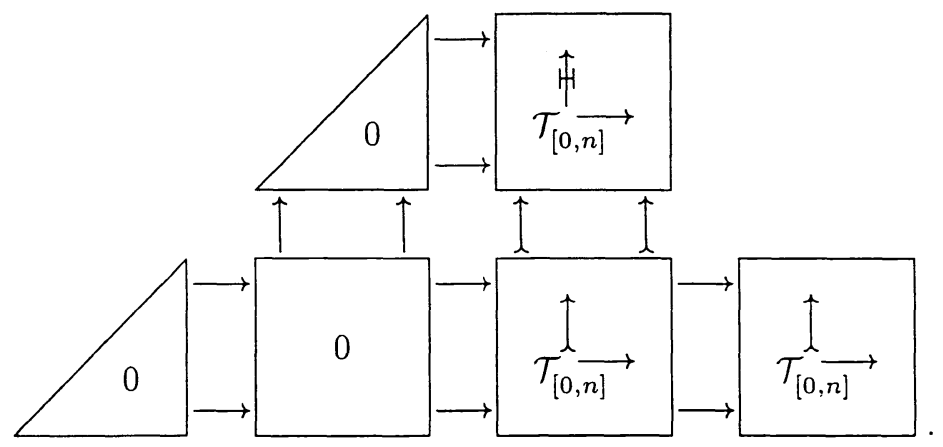

Thus, a square box with a zero inside means just that the author has been too lazy to write in what objects of $\mathcal{T}$ are permitted, and what morphisms can occur; the reader can work this out from the rest of the diagram.

It seems not implausible that the map

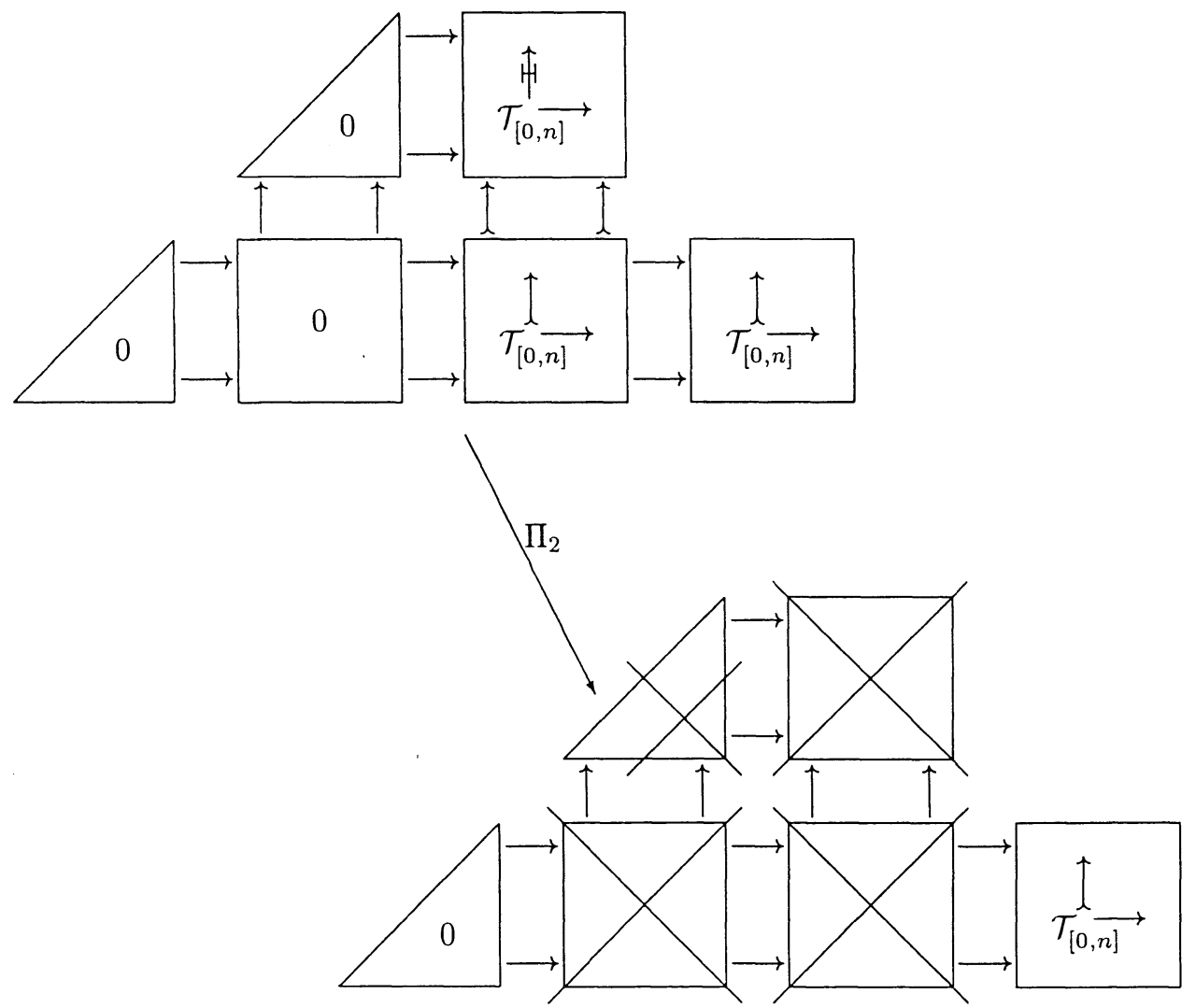

should be a quasifibration.

But then there is a commutative diagram where the columns are fibrations 

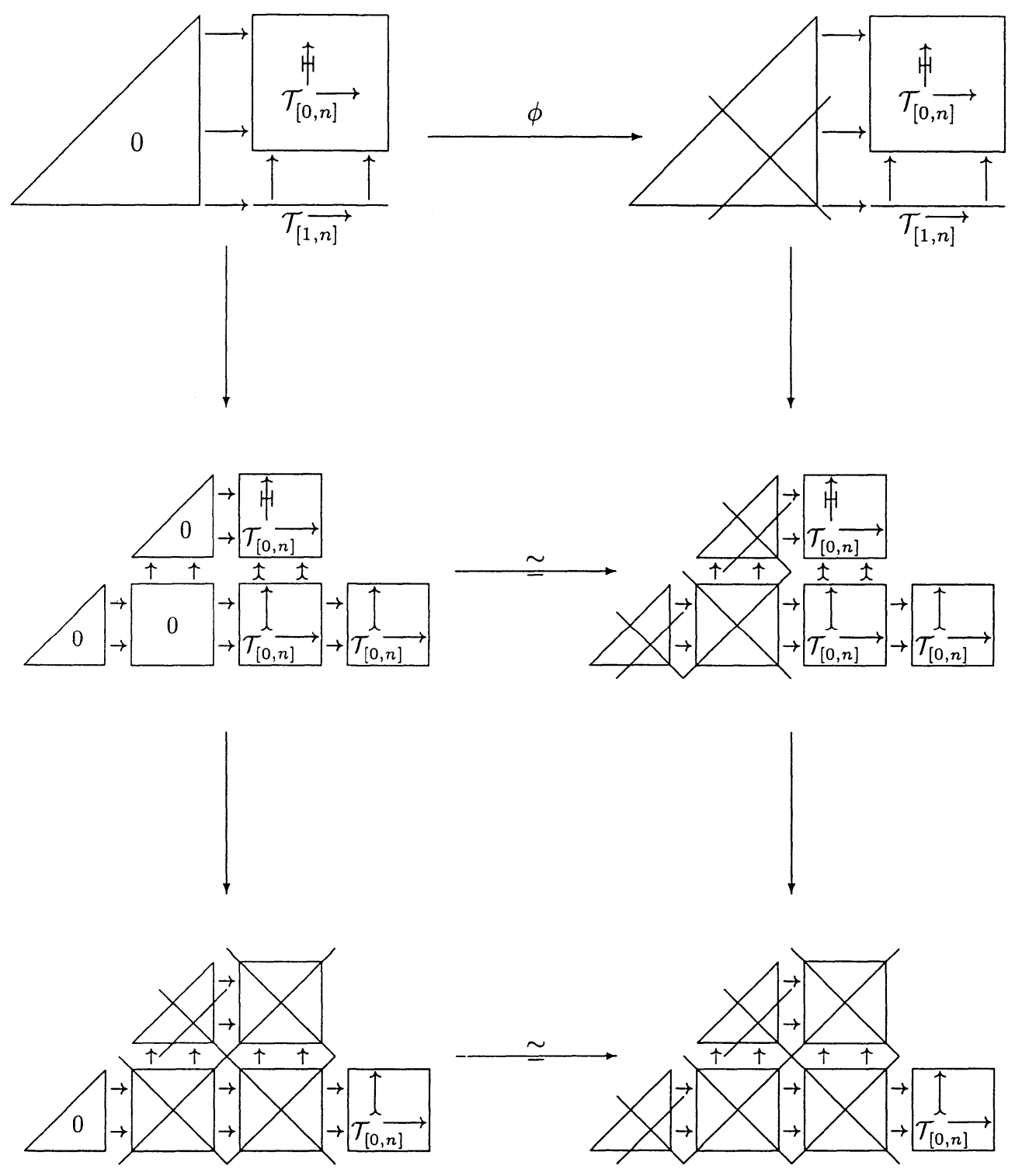

It would immediately follow that $\phi$ is a homotopy equivalence. But it is trivial to show (see Lemma 3.9) that $\phi$ is null homotopic.

As I have said before, this is the idea of the proof, but sadly I have not been able to turn this into a rigorous argument. Instead, we proceed quite indirectly to show that there are homotopy equivalences 
$(3.1)$
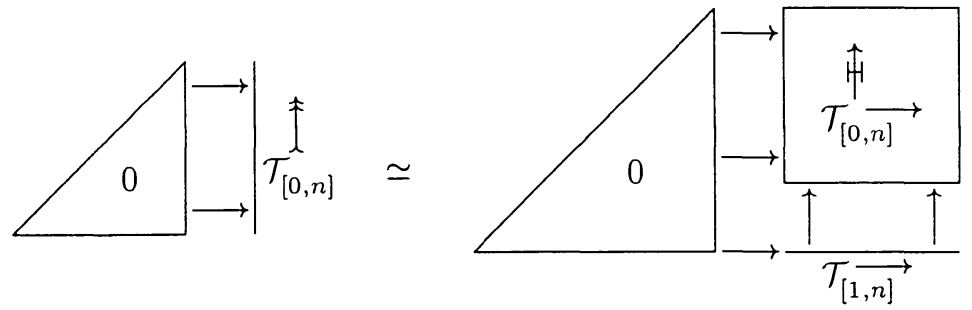

$(3.2)$

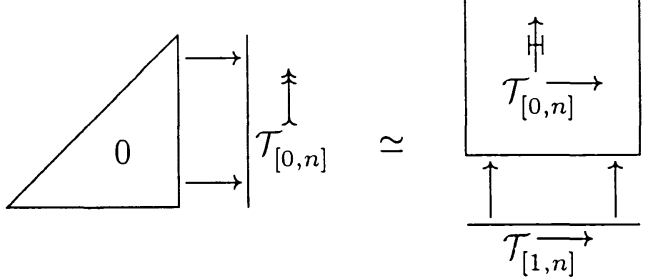

The maps 3.1 and 3.2 are natural enough so that the composite

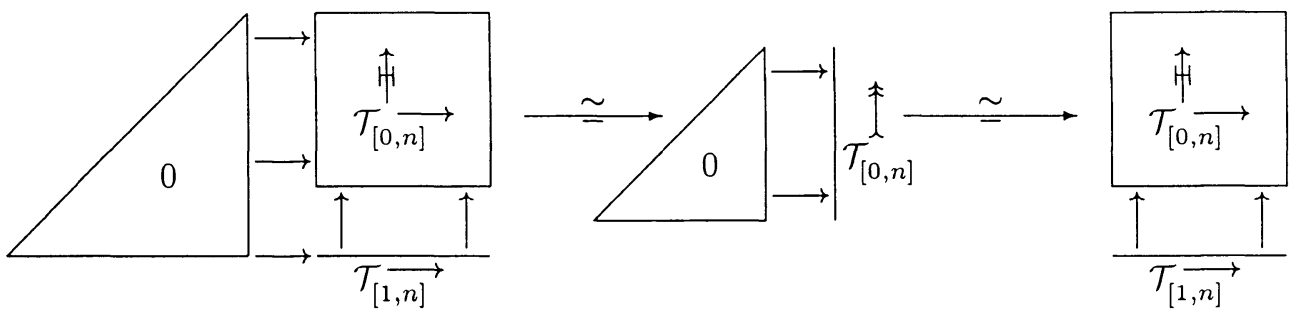

is just the map $\phi$ above. Thus, by an indirect argument we prove that $\phi$ is a homotopy equivalence, and then Lemma 3.9 establishes the contractibility of all these simplicial sets, and hence Theorem I.7.1.

Once again, the proof is reminiscent of a space shuttle mission. The space shuttle takes off from Cape Canaveral, goes many times around the earth, and then comes to land not so far from the take-off point. I sincerely hope someone succeeds in finding a simpler proof.

\section{End of Motivation}

Important Notation 3.1. . When we write the simplicial set

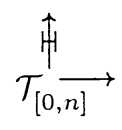

we will, as in Section I.8, mean the bisimplicial set whose $(p, q)$-simplices are diagrams 


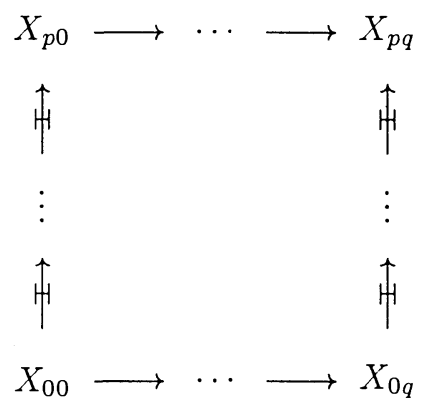

together with a coherent differential $X_{p q} \stackrel{d}{\longrightarrow} \Sigma X_{00}$. Now note that $X_{0 q} \longrightarrow X_{p q} \stackrel{d}{\longrightarrow}$ $\Sigma X_{00}$ must be zero, and because $H^{0}\left(X_{0 q}\right) \longrightarrow H^{0}\left(X_{p q}\right)$ is an isomorphism, $d$ : $X_{p q} \longrightarrow \Sigma X_{00}$ must factor through $X_{p q} \longrightarrow X_{p q}^{>0} \stackrel{d^{\prime}}{\longrightarrow} \Sigma X_{00}$. But the reader can easily see that $d^{\prime}$ is not unique.

We will sometimes want to assume that a choice of $d^{\prime}$ is as part of the structure of a simplex. We will denote this simplicial set

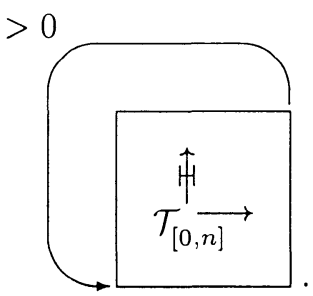

A simplex in this simplicial set is a diagram

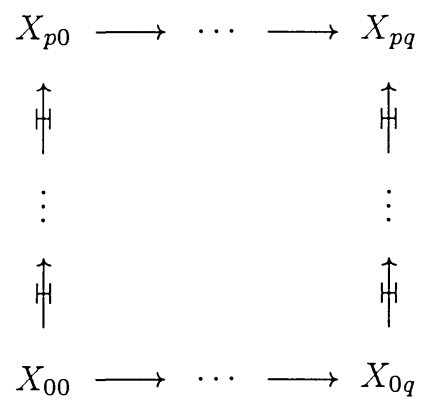

together with compatible differentials $X_{i^{\prime} j^{\prime}}^{>0} \longrightarrow \Sigma X_{i j}$ for all $i<i^{\prime}, j<j^{\prime}$. Not only that, but we further assume that everything which should be a triangle actually is, in particular, the sequence

$$
X_{i j} \longrightarrow X_{i j^{\prime}} \oplus X_{i^{\prime} j}^{>0} \longrightarrow X_{i^{\prime} j^{\prime}}^{>0} \longrightarrow \Sigma X_{i j}
$$

is a triangle.

It may help the reader to observe that, in the simplicial set

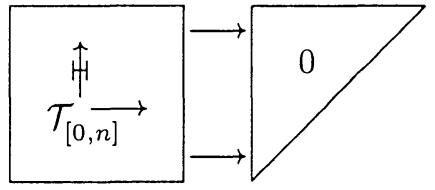


the differential $X_{p q}^{>0} \longrightarrow \Sigma X_{00}$ can be chosen canonically. A simplex is a diagram

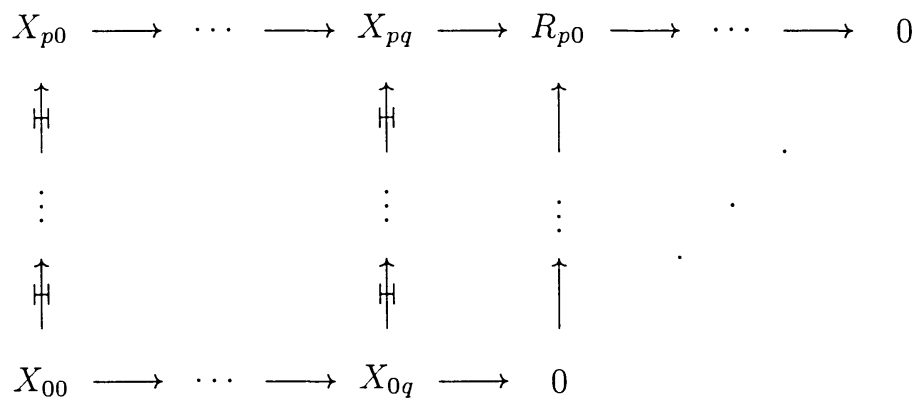

In particular, the differential $X_{p q} \longrightarrow \Sigma X_{00}$ factors through $R_{p 0}$, and because the composite $X_{p 0}^{\leq 0} \longrightarrow X_{p q} \longrightarrow R_{p 0}$ is zero, and both $X_{p q}$ and $R_{p 0}$ are in $\mathcal{T}^{\geq 0}$, the map $X_{p q} \longrightarrow R_{p 0}$ factors uniquely through $X_{p q}^{>0}$. Thus for the map $X_{p q}^{>0} \longrightarrow \Sigma X_{00}$ we simply choose the composite $X_{p q}^{>0} \longrightarrow R_{p 0} \longrightarrow \Sigma X_{00}$.

In other words, the projection

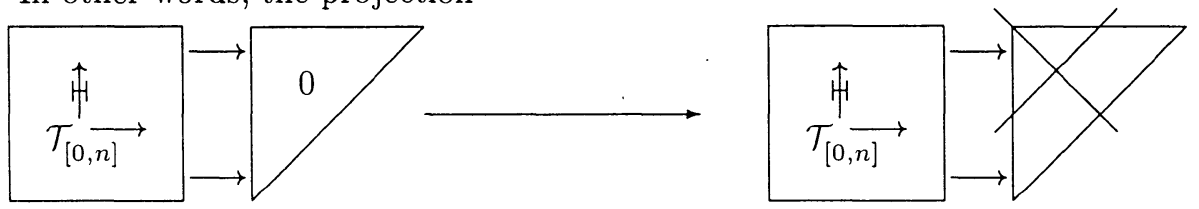

factors through

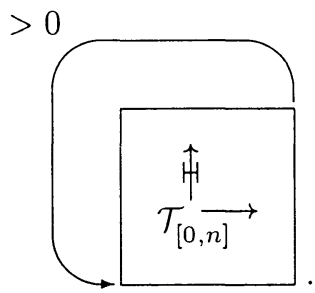

Thus the simplicial set just described is fairly natural, being intermediate between two fairly natural simplicial sets.

REMARK 3.2. The reason we have waited until this section to make this definition is that it does not affect the $G r$ constructions. Precisely, the simplicial sets

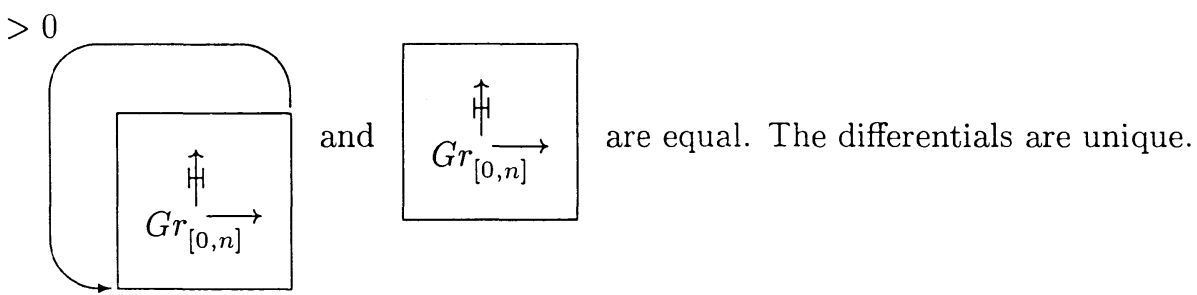

As promised, in this section we will prove the contractibility of

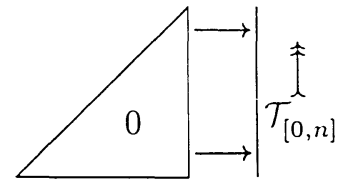


and, modulo Section I.7, this will provide a second proof of Theorem I.4.8, and a proof of Theorem I.7.1 in the special case where $\mathcal{T}=D^{b}(\mathcal{A})$ and the $t$-structure is the canonical one.

\section{Strategy Session}

We want to prove, just as in Section I.8, that the simplicial set

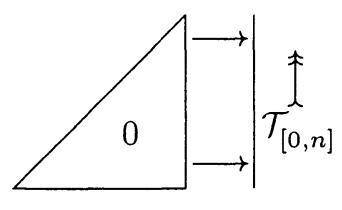

is homotopy equivalent to

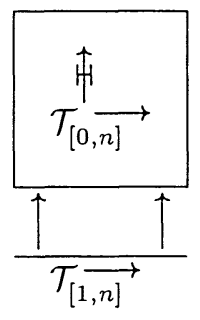

but this time the proof is rather more elaborate than it had been in Section I.7. We begin by considering a pentasimplicial set with two projections: 
$>0$
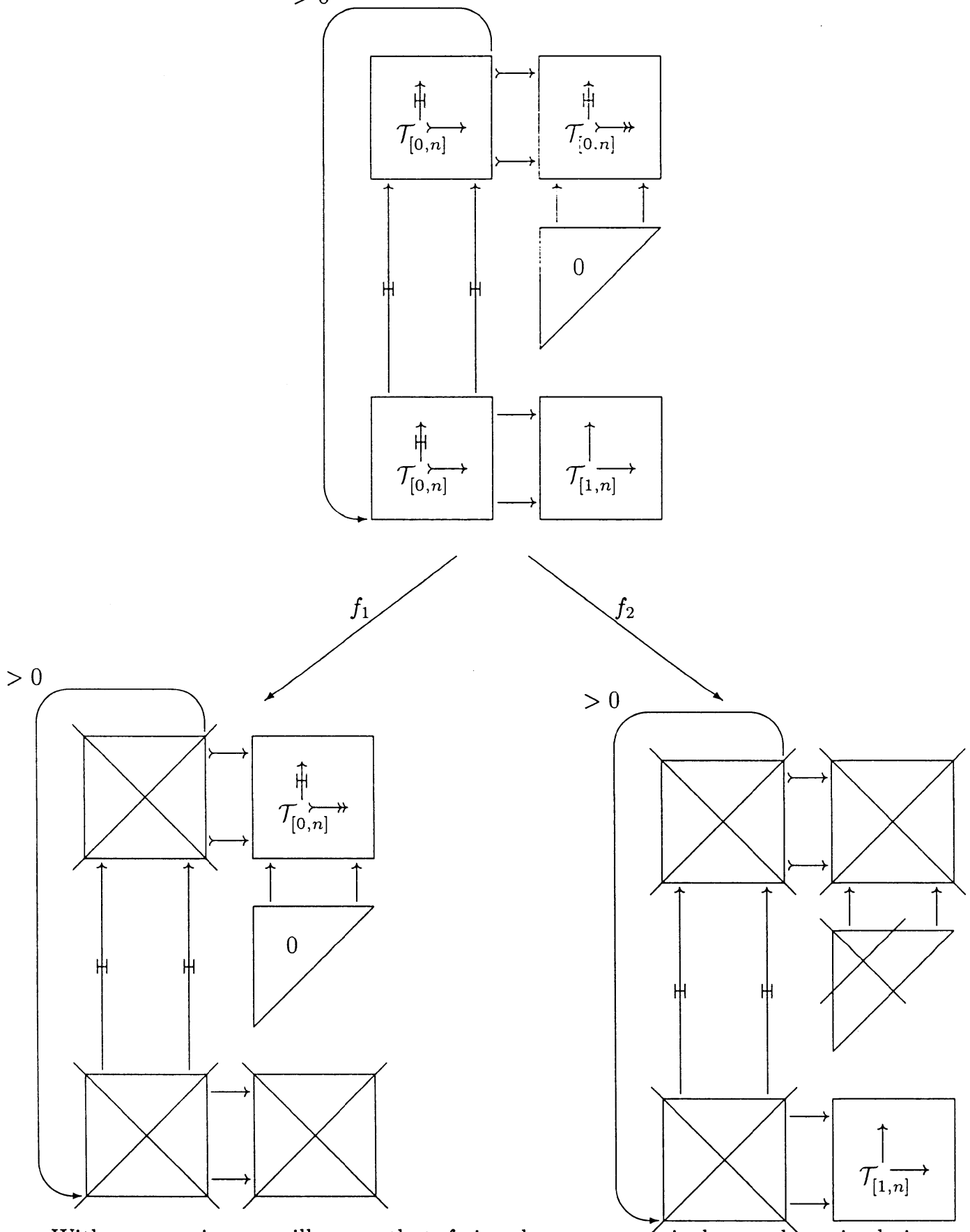

With some pain, we will prove that $f_{1}$ is a homotopy equivalence; the pain derives from the fact that some of the boxes have factorizations of differentials through the truncations, while others do not. Having gone through some unpleasantness over this point, we will have a relatively easy time showing that $f_{2}$ is a quasifibration. After all, that is just Prototype Quasifibration 1.2.

Having come this far, we will then relatively easily show that the fiber of $f_{2}$ can be identified with our favorite simplicial set 


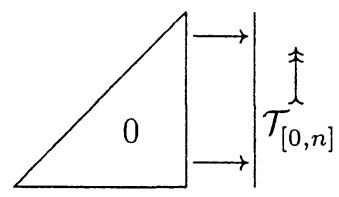

In other words, all this work will have got us precisely as far as Lemma I.8.1 did, back when we were working with good old $G r$. After that, we will follow the argument of Section I.8, which beyond Lemma I.8.1 begins working for $\mathcal{T}$ as well as for $G r$, all the way through Lemma I.8.7. Then we will have proved that there is a homotopy equivalence
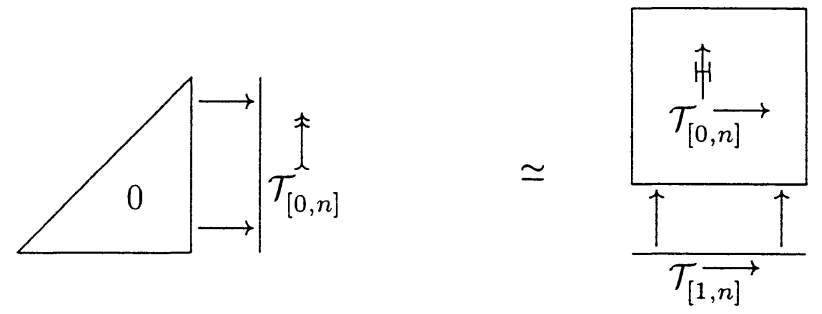

and after that it will be time for another strategic interlude.

\section{End of Strategic Planning.}

IMPORTANT NOTATION 3.3. . The simplicial set

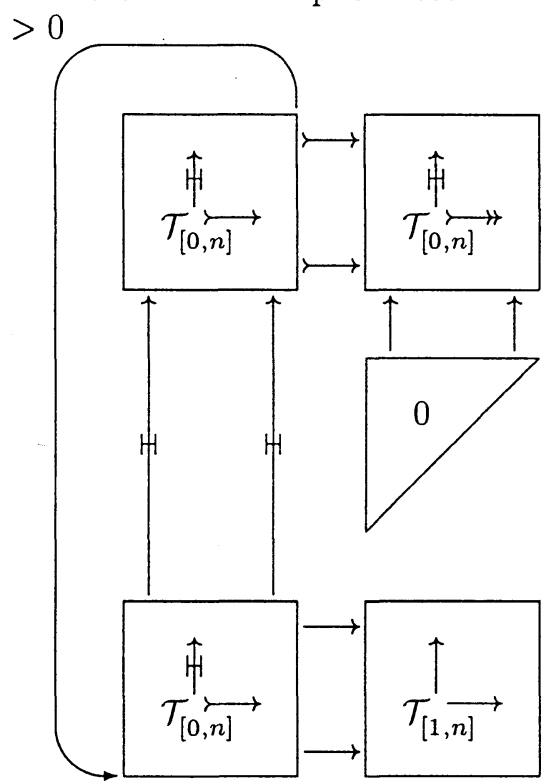

needs some explanation. Following the notation introduced in Important Notation 3.1, we have a clear idea that on the left hand side of the simplicial set, we would expect to be given a factorisation of the differential through a truncation. But how is this to be compatible with the other differentials in the diagram? Is there a compatibility requirement?

A simplex in 

$>0$

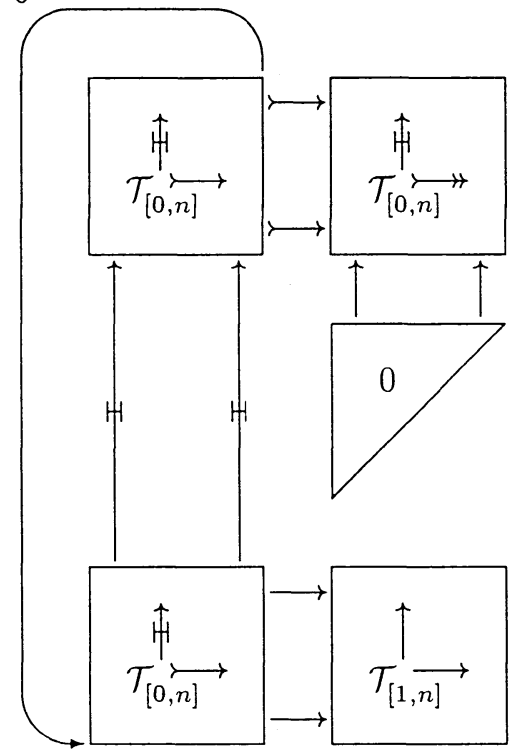




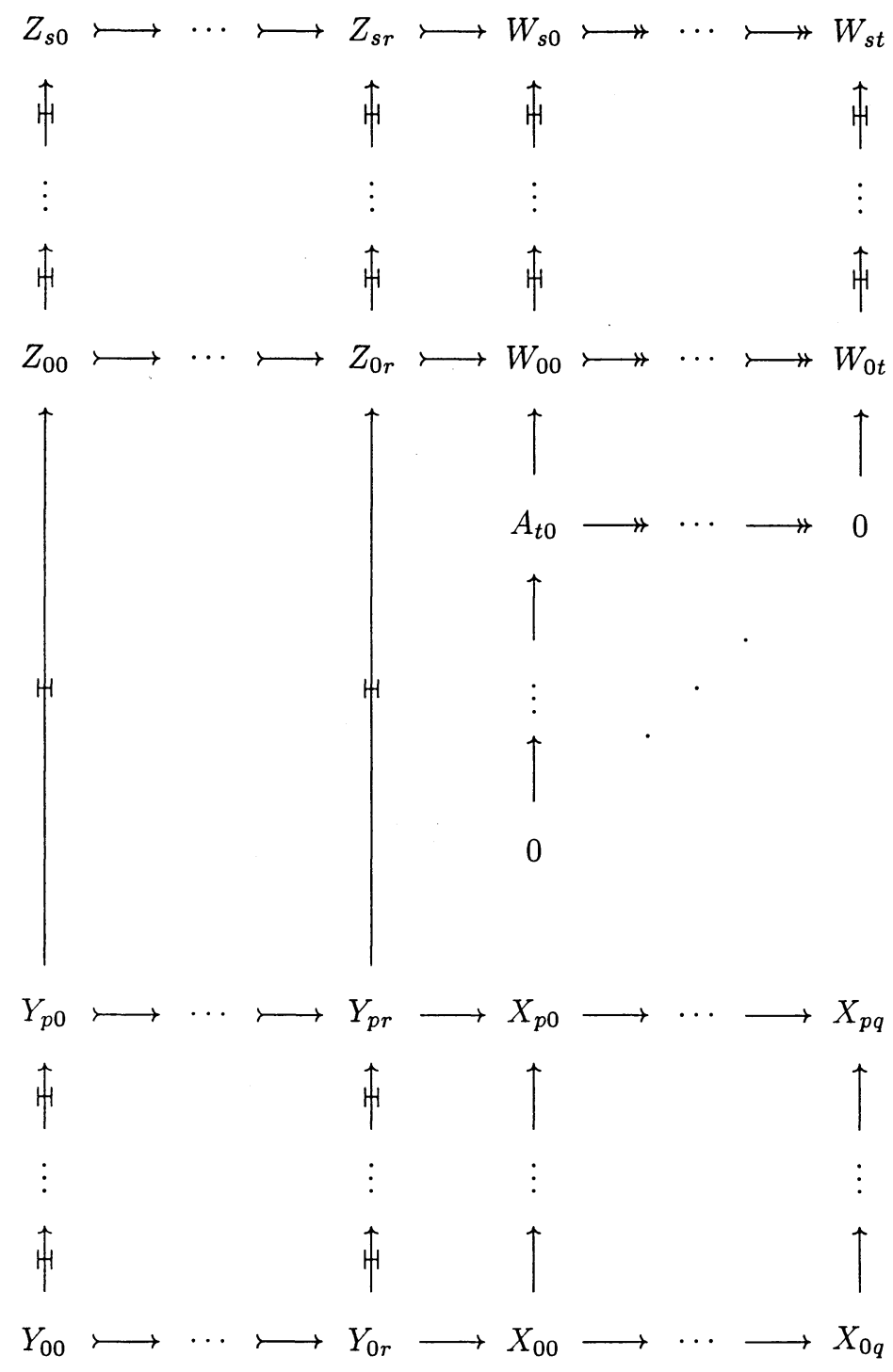

together with some differentials. In particular, we have a differential $Z_{s q}^{>0} \longrightarrow \Sigma Y_{00}$, and some other less exotic differentials that do not involve truncations. What compatibilities should we impose, especially on the exotic, truncated differentials?

There is a differential $Y_{p r}^{>0} \longrightarrow \Sigma Y_{00}$, which is "exotic." There is a straight differential $X_{p 0} \longrightarrow \Sigma Y_{00}$. Because $X_{p 0}$ is an object of $\mathcal{T}_{[1, n]}$, the map $Y_{p r} \longrightarrow X_{p 0}$ factors canonically as $Y_{p r} \longrightarrow Y_{p r}^{>0} \longrightarrow X_{p 0}$. The composite $Y_{p r}^{>0} \longrightarrow X_{p 0} \longrightarrow \Sigma Y_{00}$ should clearly be assumed equal to the "exotic" differential.

Less clear is what hypothesis to place on the compatibility of the "exotic" differential $Z_{s q}^{>0} \longrightarrow \Sigma Z_{00}$ with the straight differentials $W_{s j} \longrightarrow \Sigma Z_{00}$. What we assume is the following. Choose integers $0 \leq i \leq i^{\prime} \leq s$, and $0 \leq j \leq t$. There is a map $Z_{i^{\prime} q} \oplus W_{i j} \stackrel{\Delta}{\longrightarrow} W_{i^{\prime} j}$. This map factors uniquely through 


$$
Z_{i^{\prime} q} \oplus W_{i j} \longrightarrow \frac{Z_{i^{\prime} q} \oplus W_{i j}}{H^{0}\left(Z_{i^{\prime} q}\right)} \longrightarrow W_{i^{\prime} j}
$$

Then what is the composite

$$
\frac{Z_{i^{\prime} q} \oplus W_{i j}}{H^{0}\left(Z_{i^{\prime} q}\right)} \longrightarrow W_{i^{\prime} j} \longrightarrow \Sigma Z_{i 0} ?
$$

There is, of course, another way to go. We have a projection

$$
\frac{Z_{i^{\prime} q} \oplus W_{i j}}{H^{0}\left(Z_{i^{\prime} q}\right)} \rightarrow \frac{Z_{i^{\prime} q} \oplus W_{i j}}{H^{0}\left(Z_{i^{\prime} q}\right) \oplus H^{0}\left(W_{i j}\right)}=Z_{i^{\prime} q}^{>0} \oplus W_{i j}^{>0}
$$

And not amazingly, we would expect the differential above to agree with the composite

$$
\frac{Z_{i^{\prime} q} \oplus W_{i j}}{H^{0}\left(Z_{i^{\prime} q}\right)} \longrightarrow Z_{i^{\prime} q}^{>0} \oplus W_{i j}^{>0} \stackrel{\Pi}{\longrightarrow} Z_{i^{\prime} q}^{>0} \stackrel{d}{\longrightarrow} Z_{i 0}
$$

where $\Pi$ is the projection and $d$ is the exotic differential.

We assume that these are equal.

REMARK 3.4. The point of this last assumption is the following. At some time in the very near future we will want to consider the homotopy

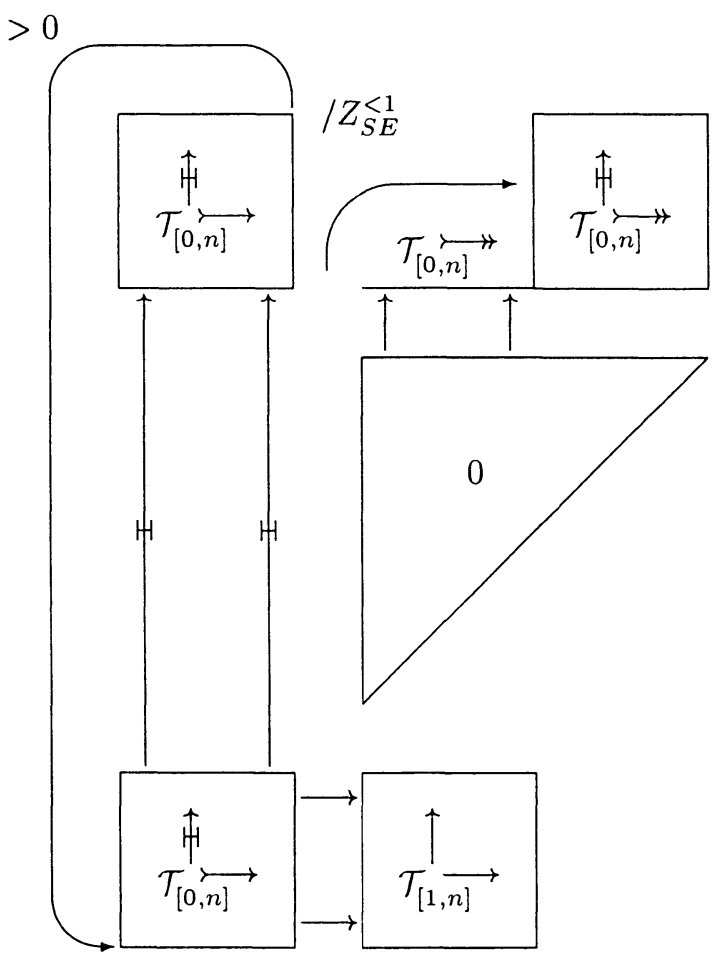

There is nothing wrong with the homotopy, ever; but unless we are careful, the end map 


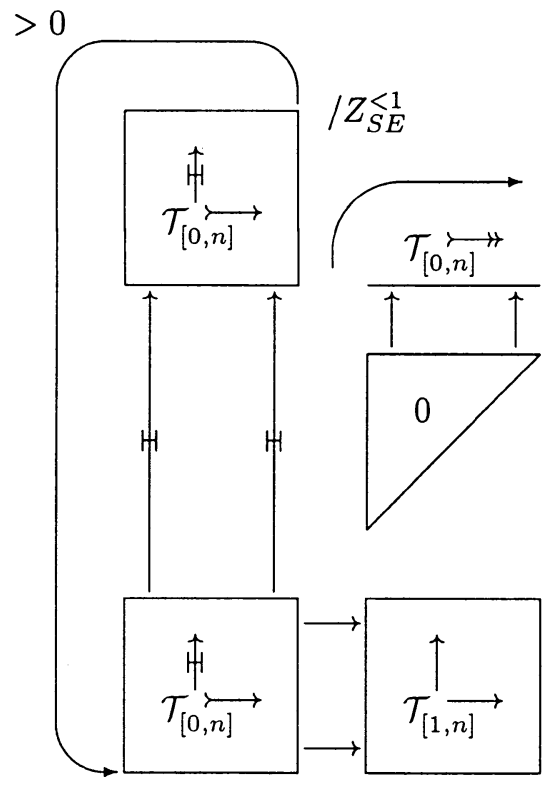

will not factor through the product
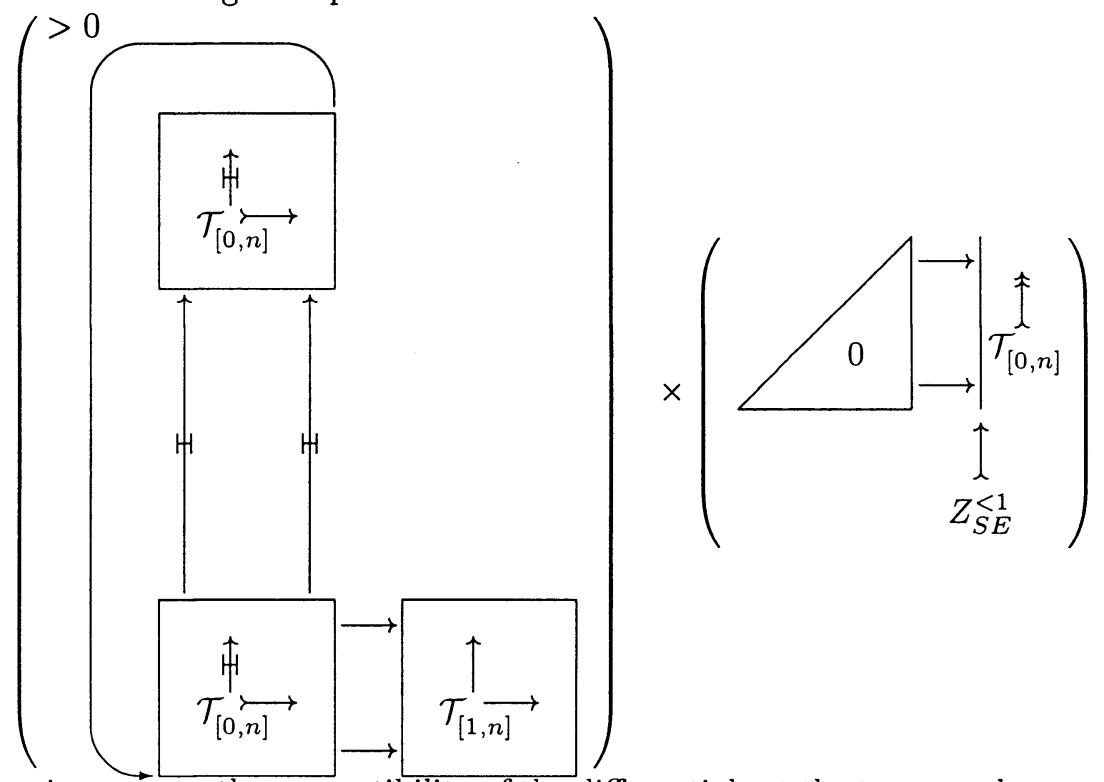

The requirement on the compatibility of the differentials at the top exactly guarantees that the differential

$$
\frac{Z_{s q} \oplus W_{0 t}}{Z_{S W}^{<1}} \rightarrow \Sigma Z_{00}
$$

is the natural map; it is the composite

$$
\frac{Z_{s q} \oplus W_{0 t}}{Z_{S W}^{<1}} \longrightarrow \frac{Z_{s q} \oplus W_{0 t}}{Z_{S W}^{<1} \oplus W_{0 t}^{<1}} \stackrel{\Pi}{\longrightarrow} Z_{s q}^{>0} \stackrel{d}{\longrightarrow} \Sigma Z_{00}
$$

LEMMA 3.5. In the diagram 

$>0$
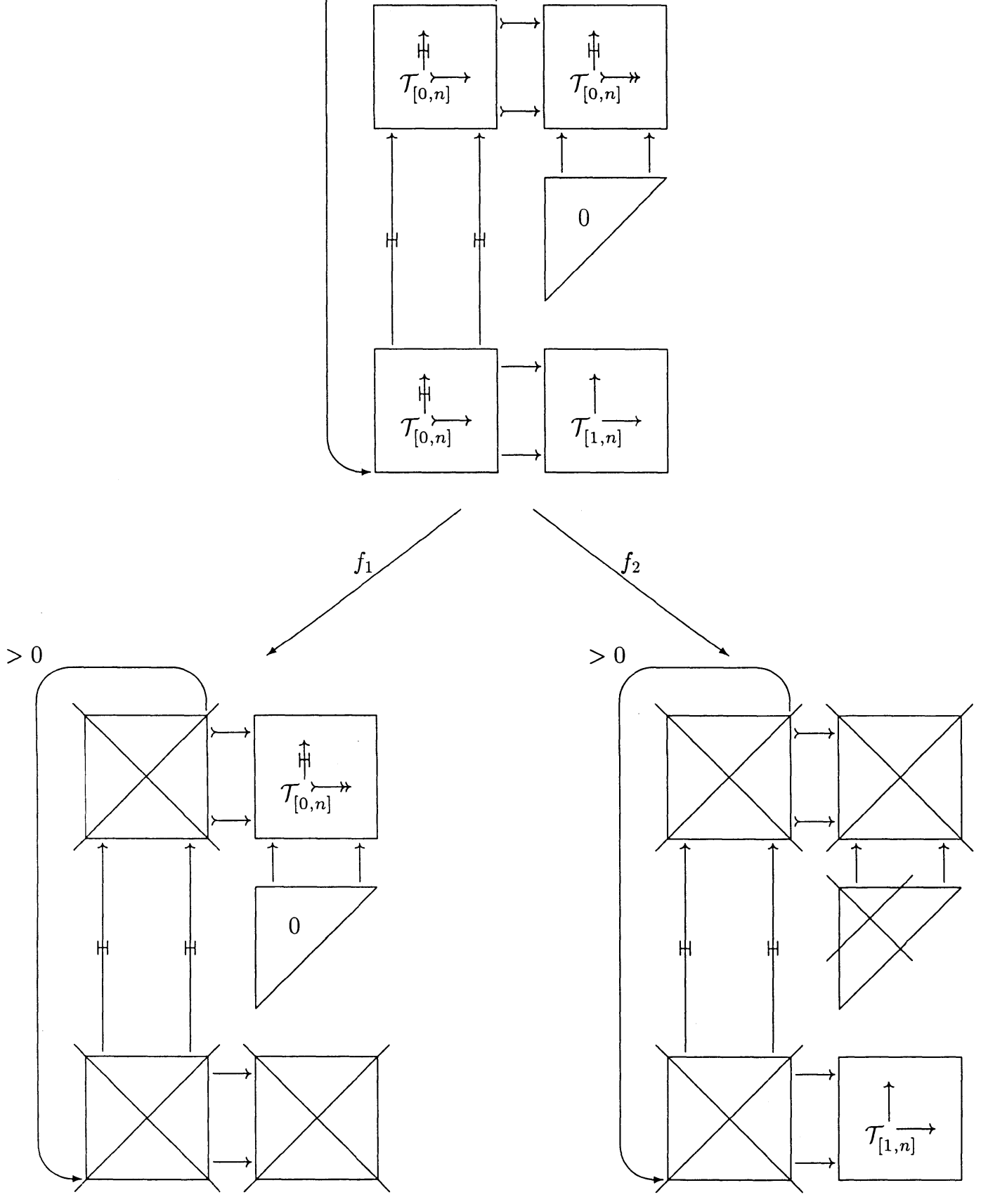

the map $f_{1}$ is a homotopy equivalence.

Proof. $f_{1}$ can be factored as the composite 


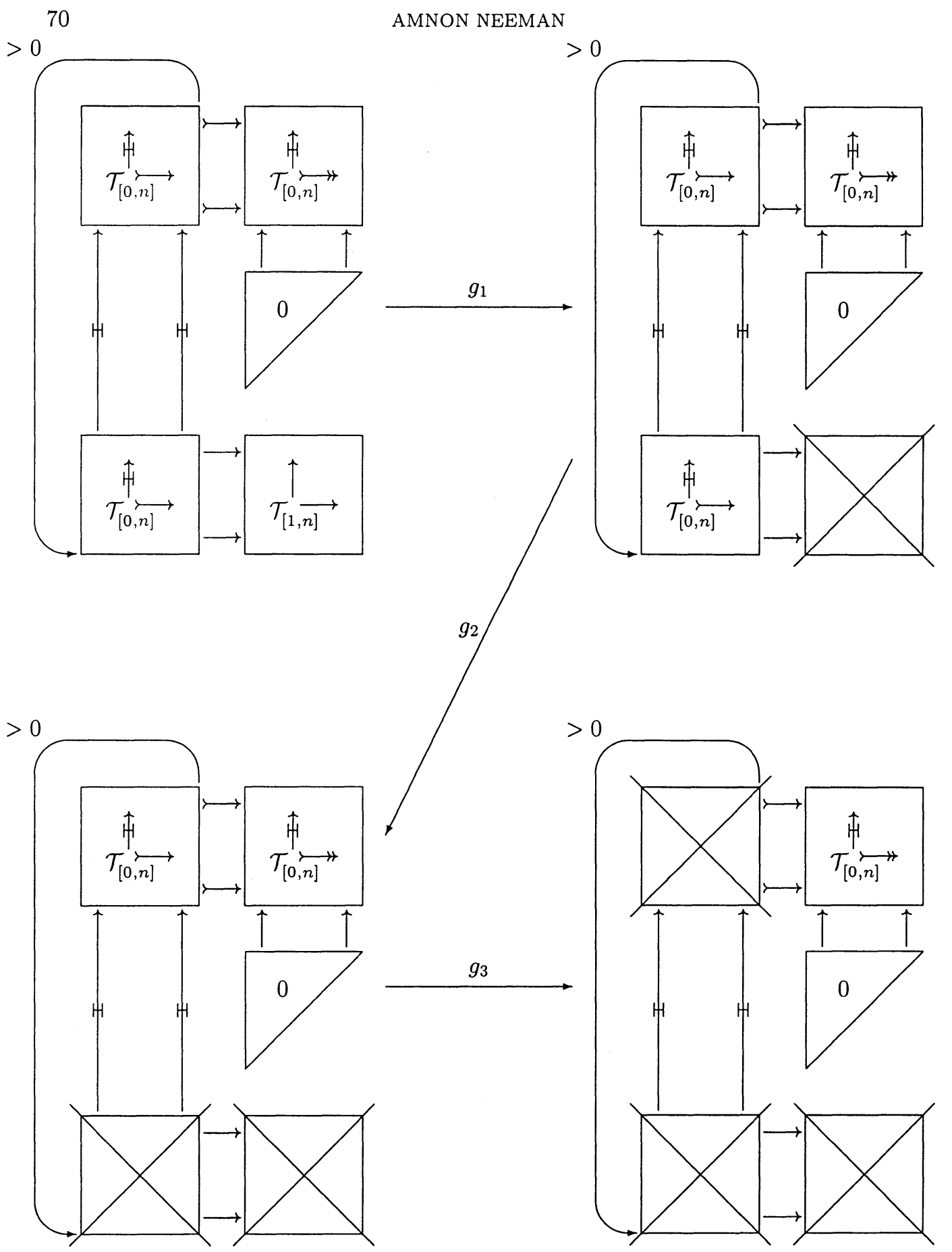

We want to prove that each of $g_{1}, g_{2}$ and $g_{3}$ is a homotopy equivalence. The difficult step is $g_{3}$, so we will treat it last.

\subsection{1. $g_{1}$ is a homotopy equivalence.}

Proof. It suffices to prove the contractibility of the Segal fiber 


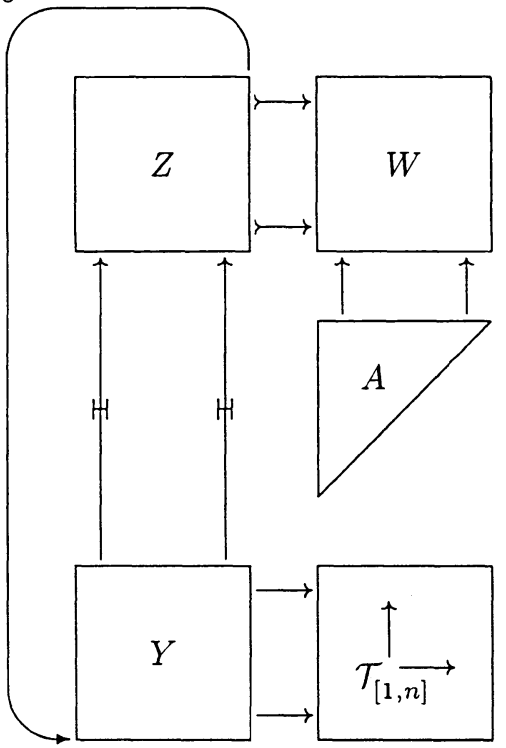

and the idea is to use the homotopy

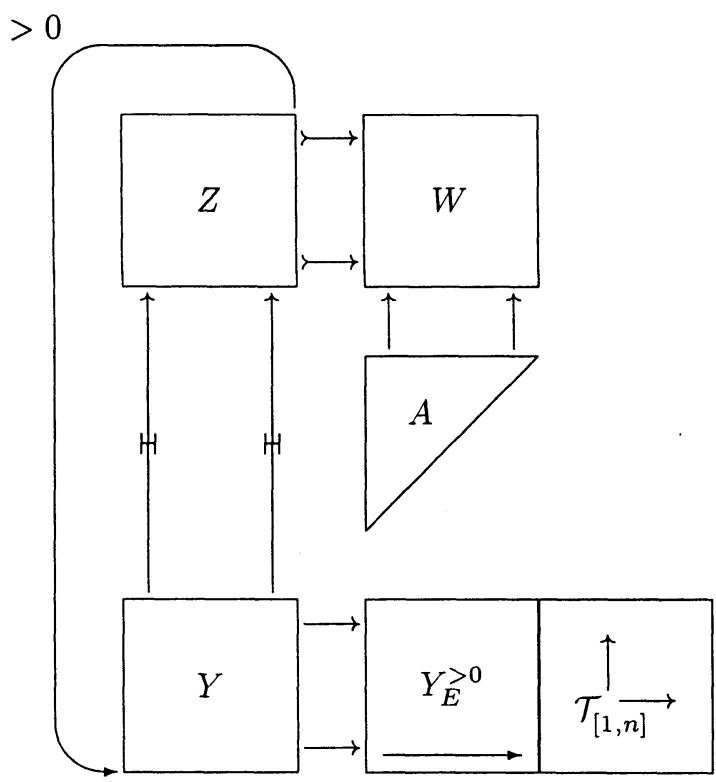

which is certainly well-defined. But it is also a contracting homotopy. It clearly is a homotopy. The fact that it contracts follows because the simplicial set contains a choice of differentials $Y_{N E}^{>0} \rightarrow \Sigma Y_{S W}$, and by hypothesis this is compatible with the differentials out of the bottom right box.

\subsection{2. $g_{2}$ induces a homotopy equivalence.}

Proof. Once again, it suffices to prove the Segal fiber 


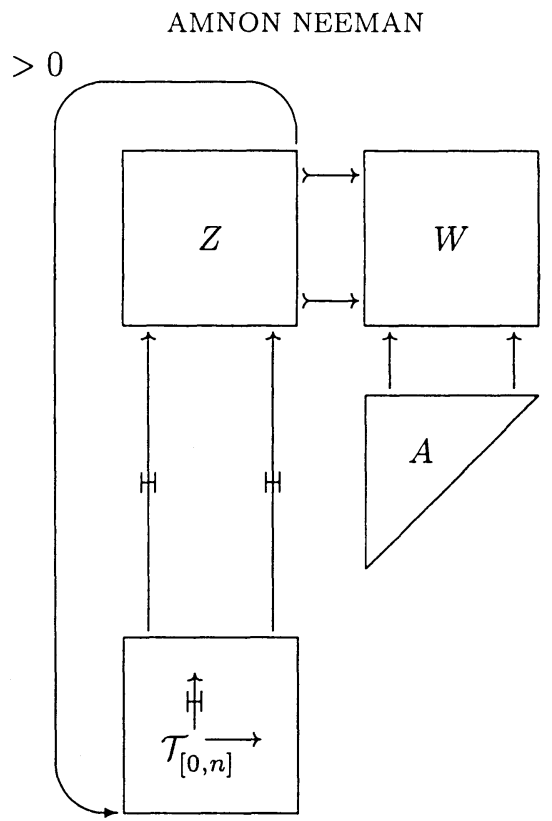

contractible. But this follows immediately from the contracting homotopy

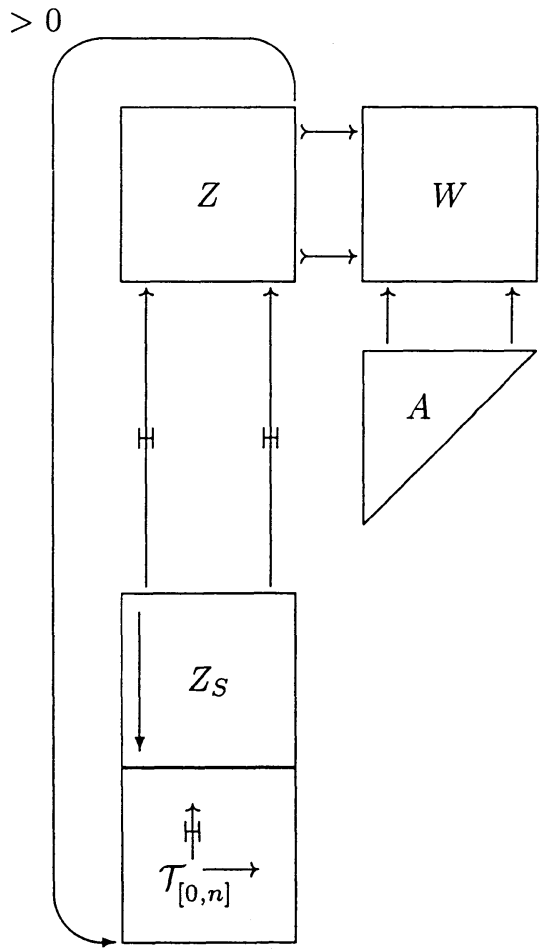

The subtle point is therefore to prove that $g_{3}$ induces a homotopy equivalence.

3.5.3. The map $g_{3}$ induces a homotopy equivalence.

Proof. We wish to show that the projection 

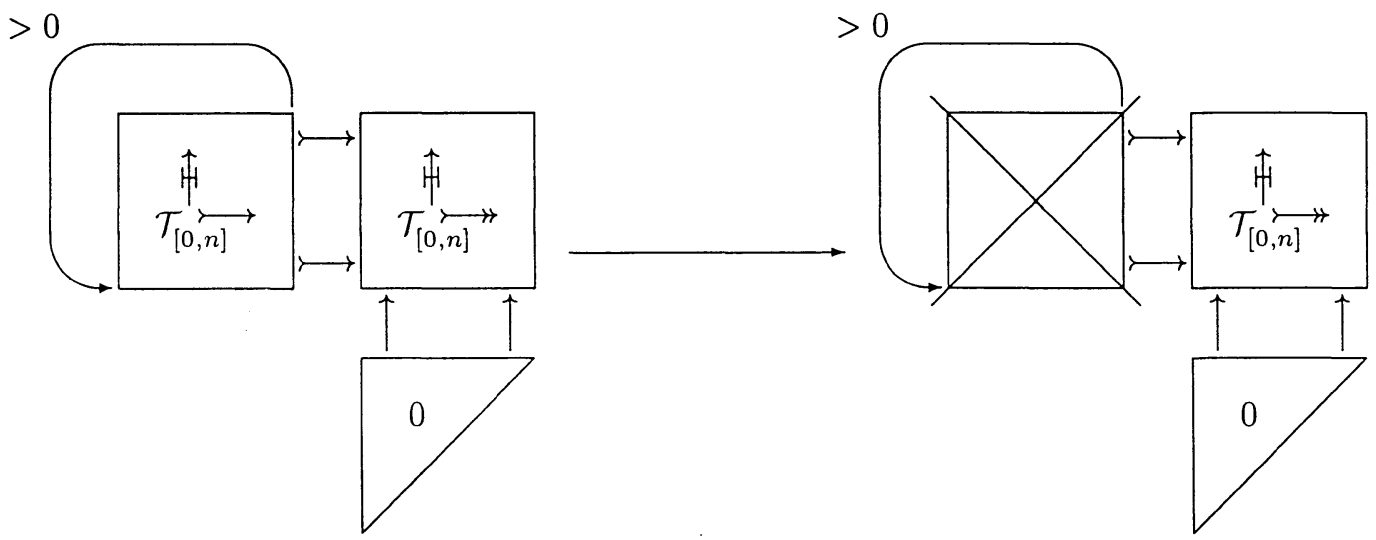

induces a homotopy equivalence. We will break it into two lemmas, showing in turn that the projections

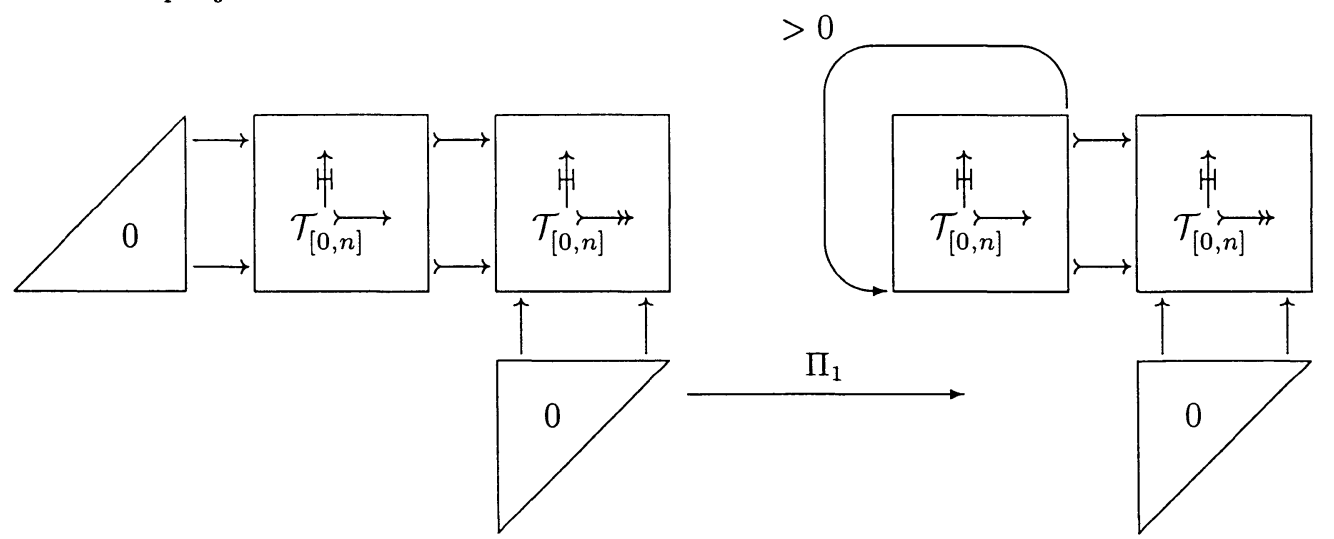

and

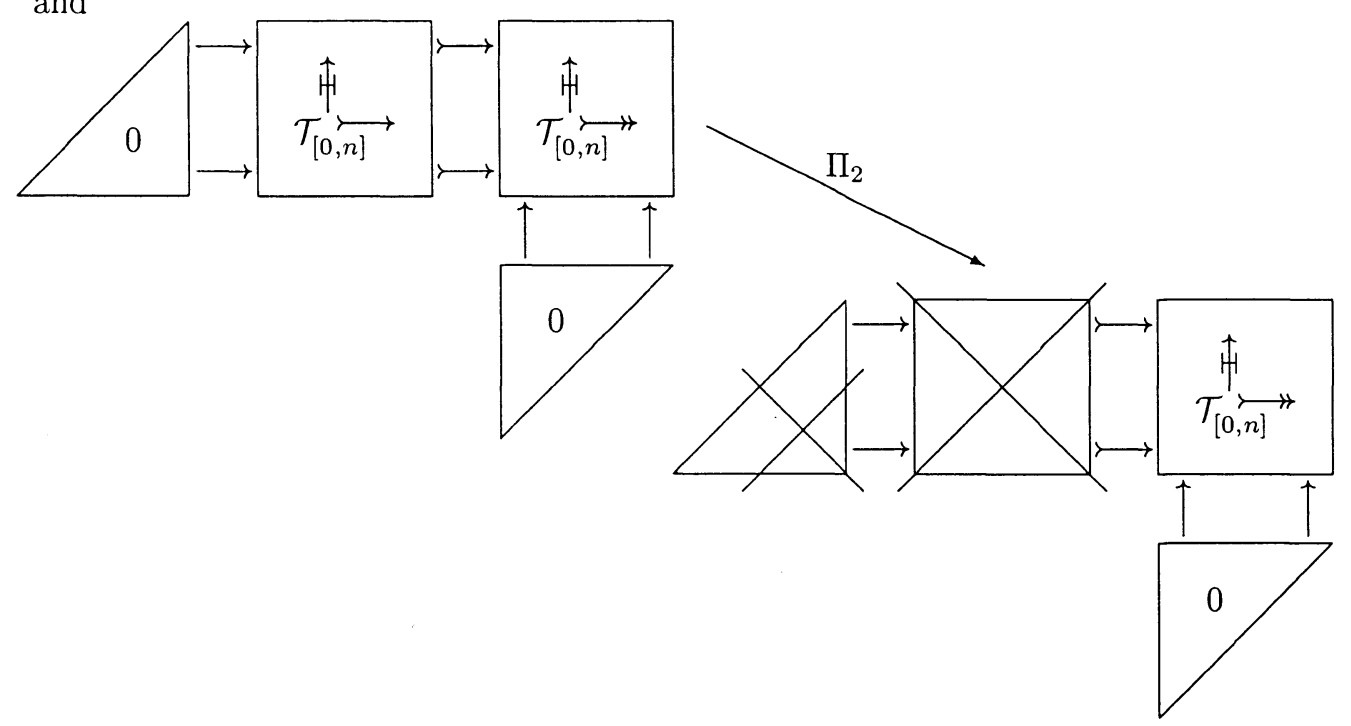

both induce homotopy equivalences. Note of course that $\Pi_{1}$ is well-defined, because the choice of kernels implicitly carries with it the choice of differentials from the truncation.

3.5.3.1. Proof that $\Pi_{1}$ is a homotopy equivalence. We need to prove that 
the map

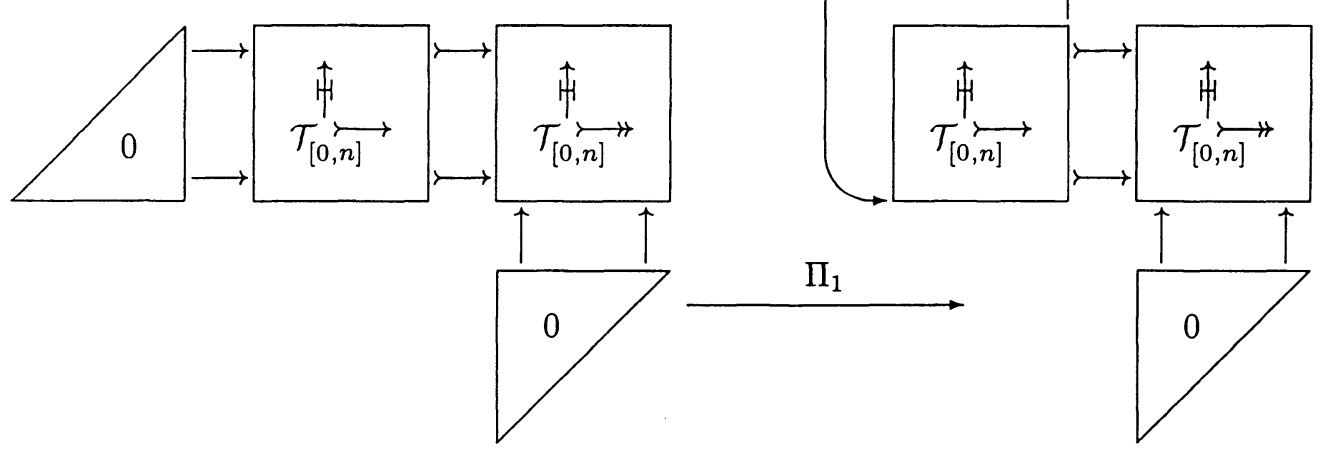

induces a homotopy equivalence.

Proof. We will instead consider the more complicated diagram 

$>0$

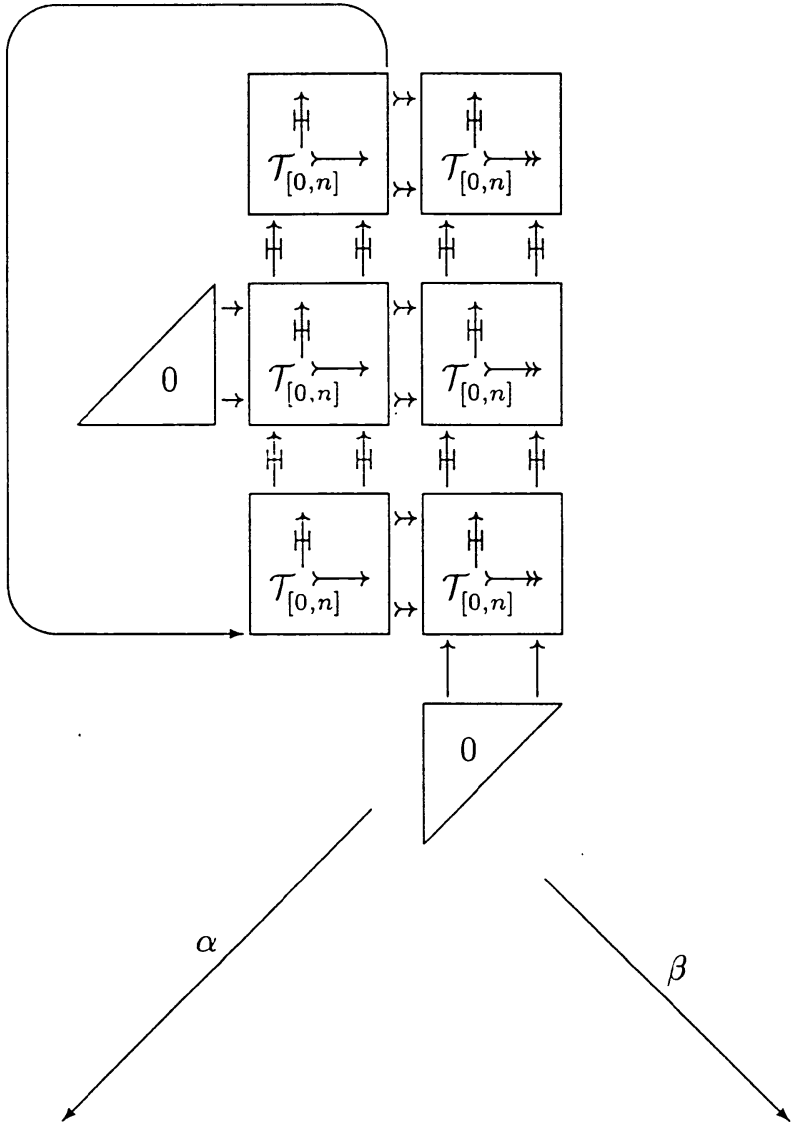

$>0$

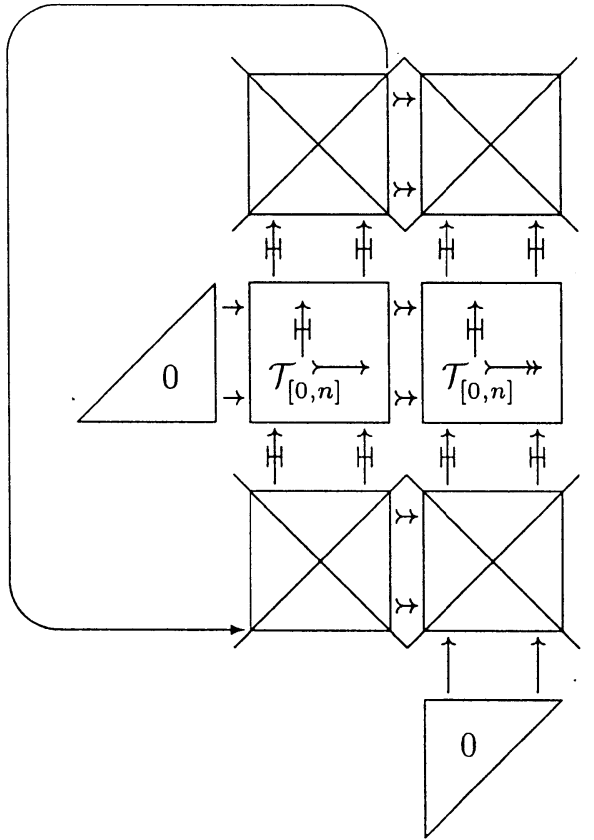

$>0$

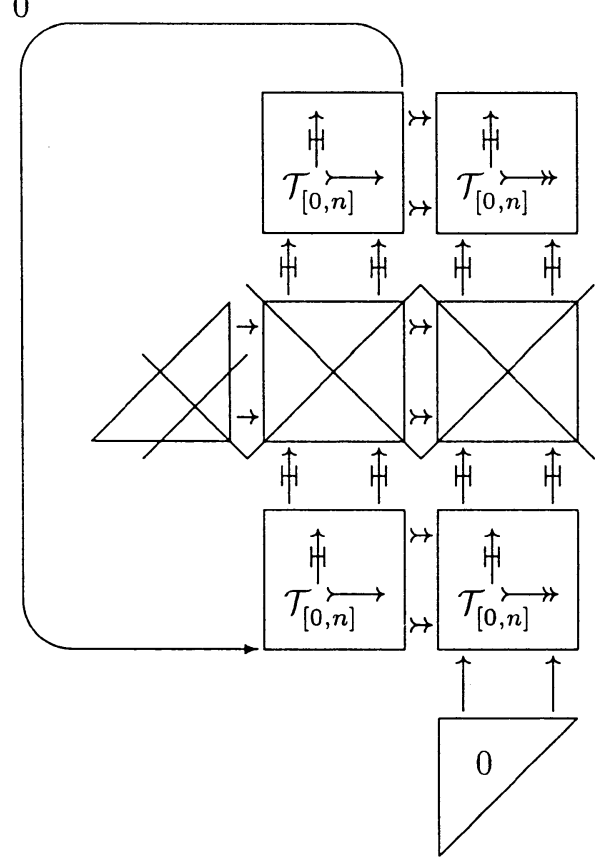


The fact that $\alpha$ induces a homotopy equivalence is essentially trivial. The Segal fiber is the simplicial set

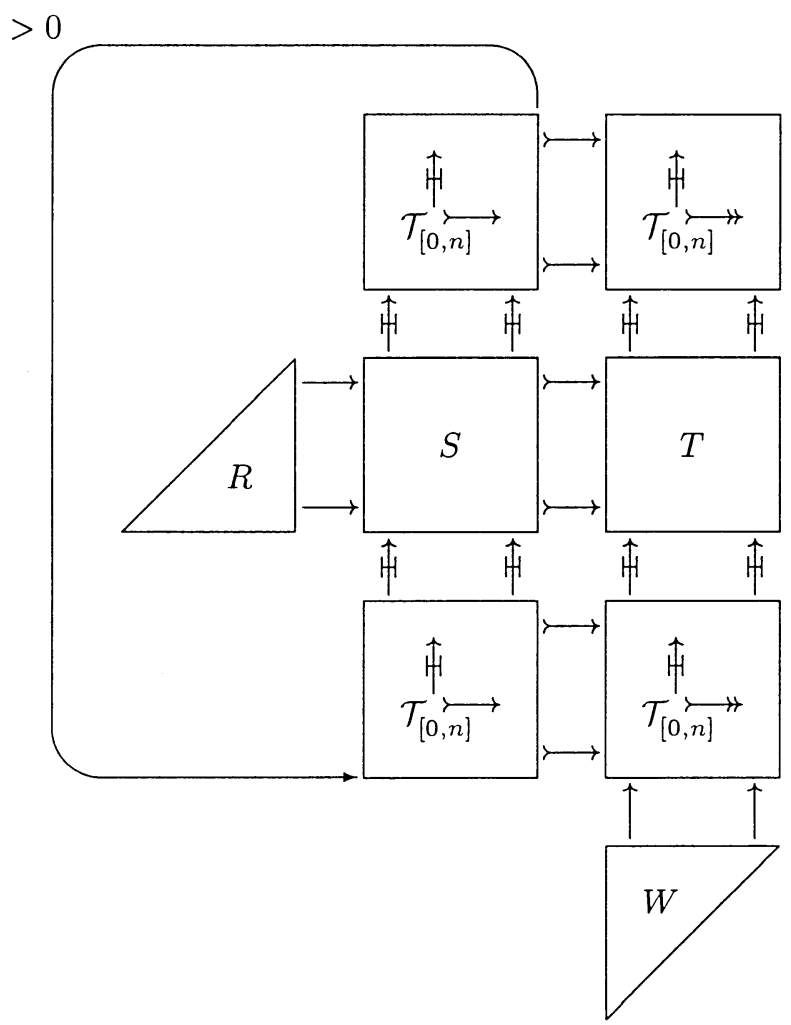

and it is contracted by the homotopy 


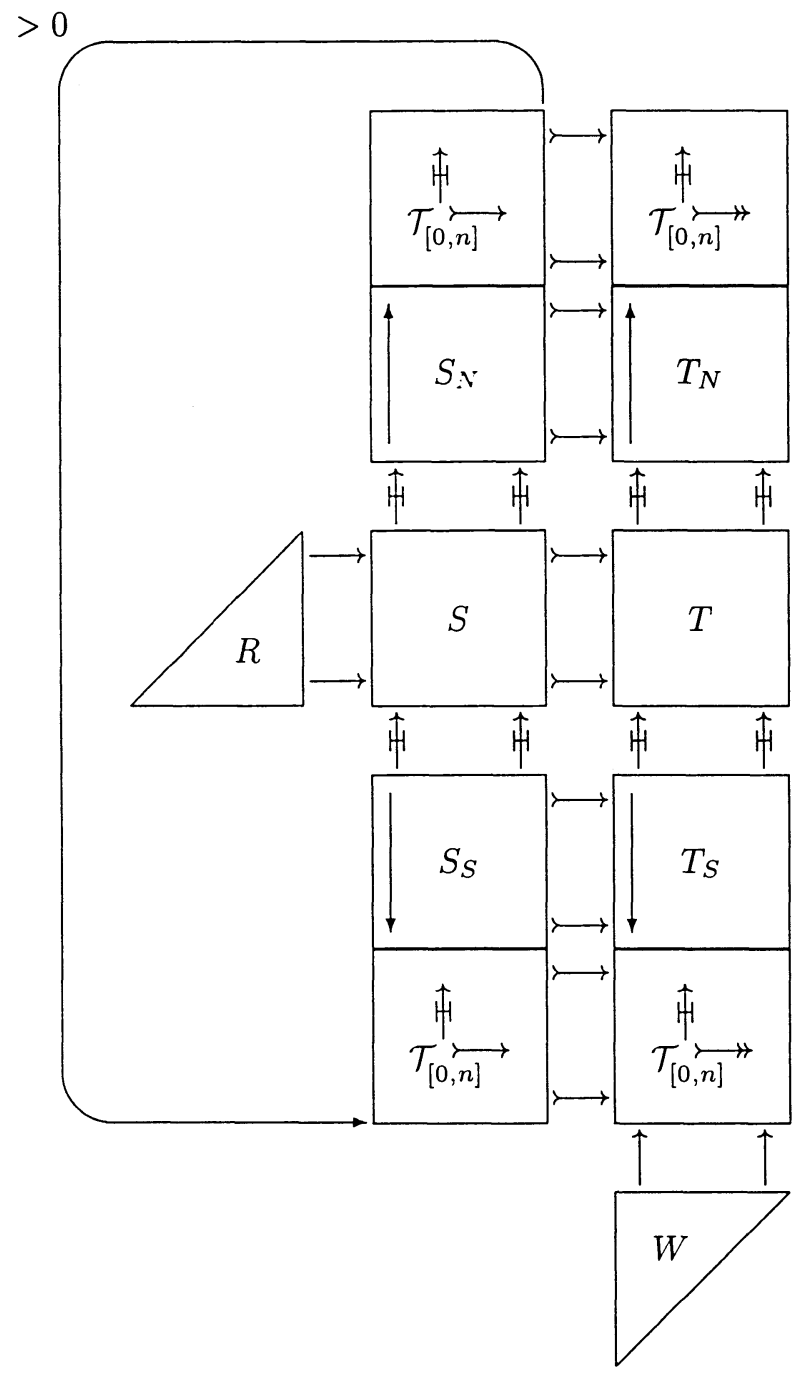

The fact that $\beta$ induces a homotopy equivalence is a little more subtle. The Segal fiber is the simplicial set 


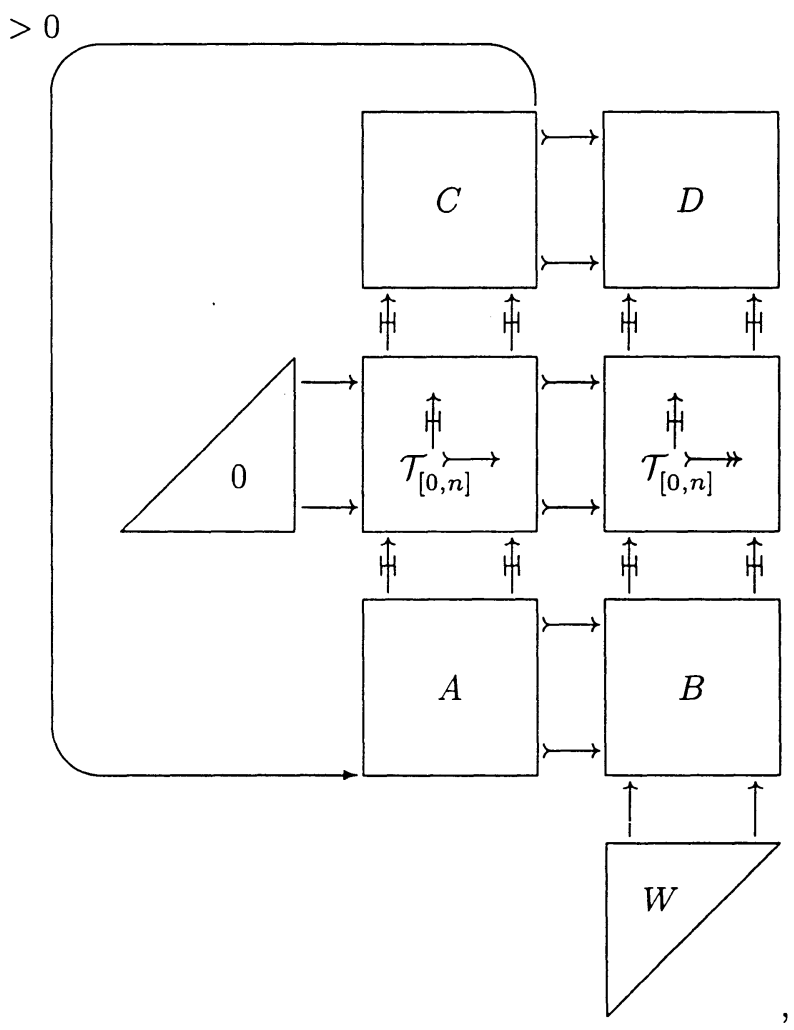

and the point of the proof is that the homotopy 


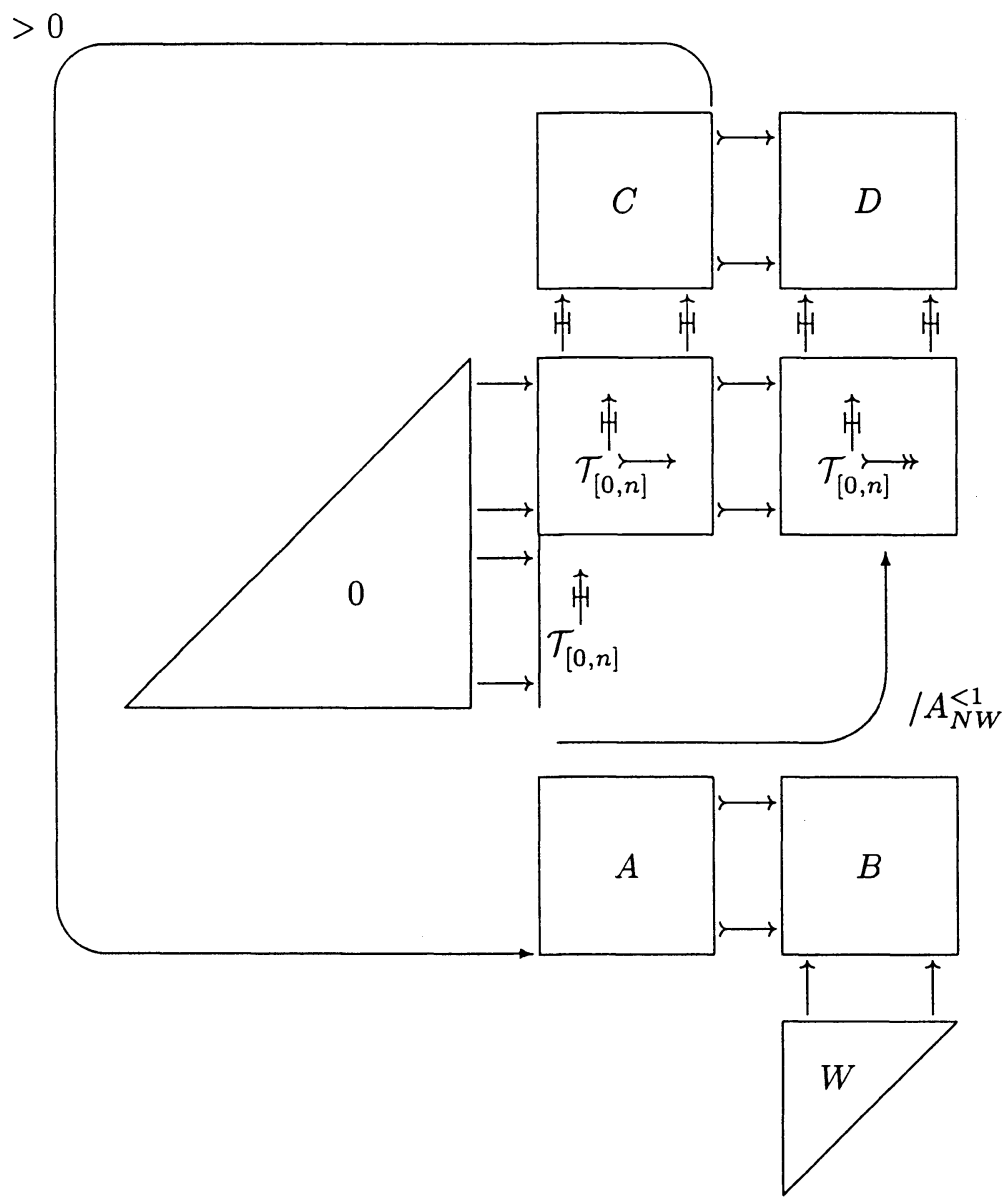

shows that, up to homotopy, the identity on the simplicial set 


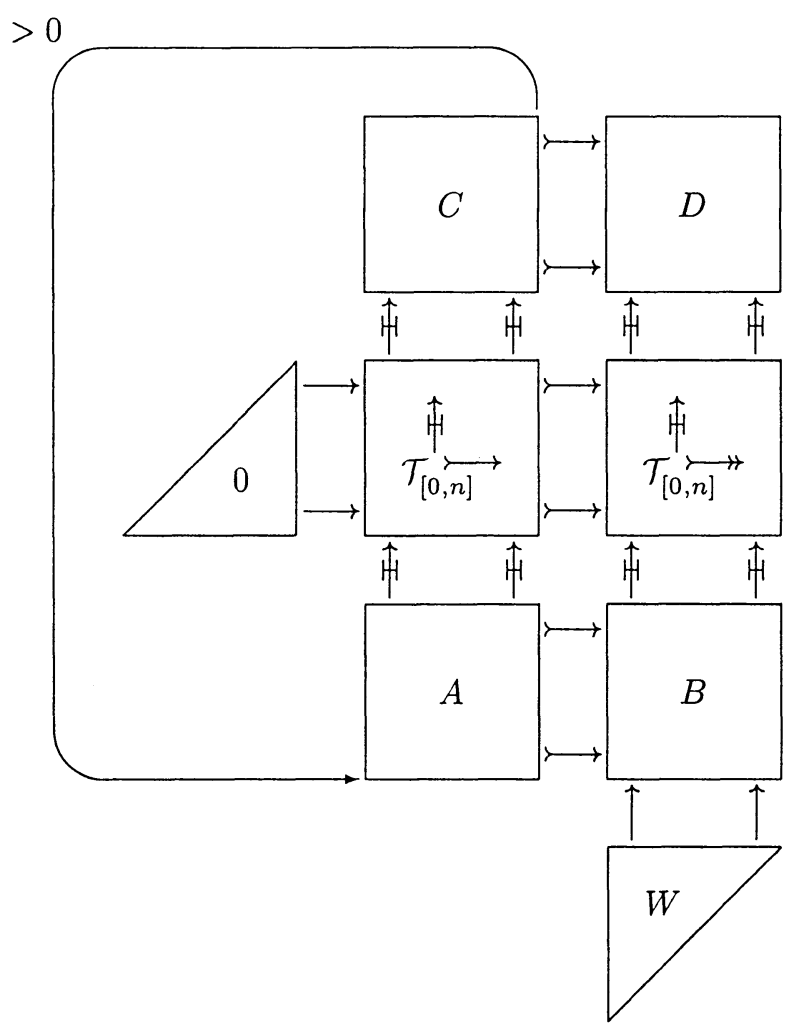

factors through the simplicial set

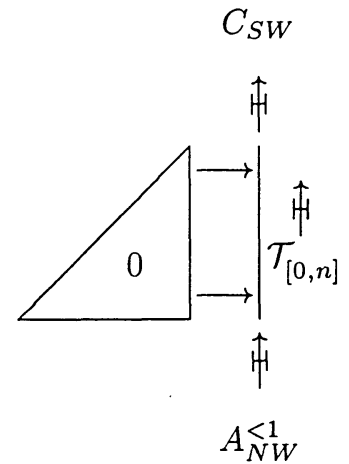

and this last simplicial set is contracted by the contraction to the initial object $A_{N W}^{<1}$. This completes the proof of Lemma 3.5.3.1.

3.5.3.2. Proof that $\Pi_{2}$ is a homotopy equivalence. We need to prove that the map 


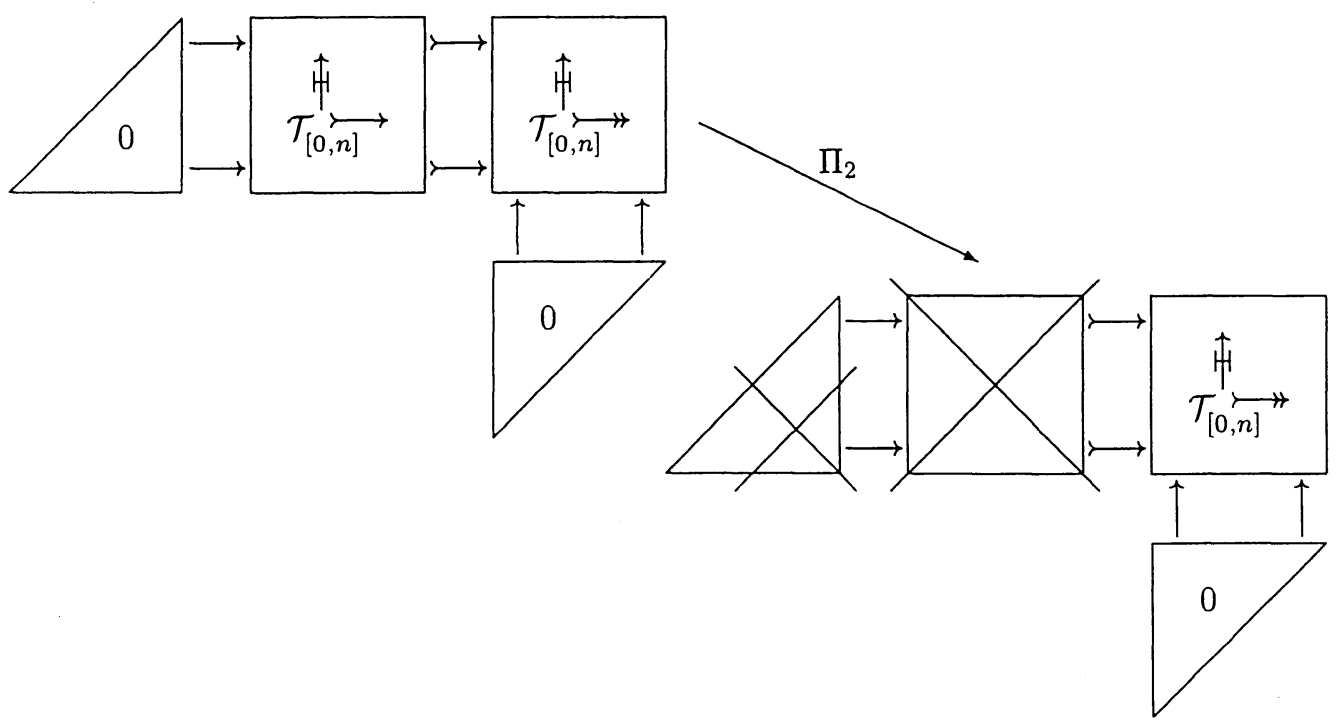

induces a homotopy equivalence.

Proof. Observe first that the natural map
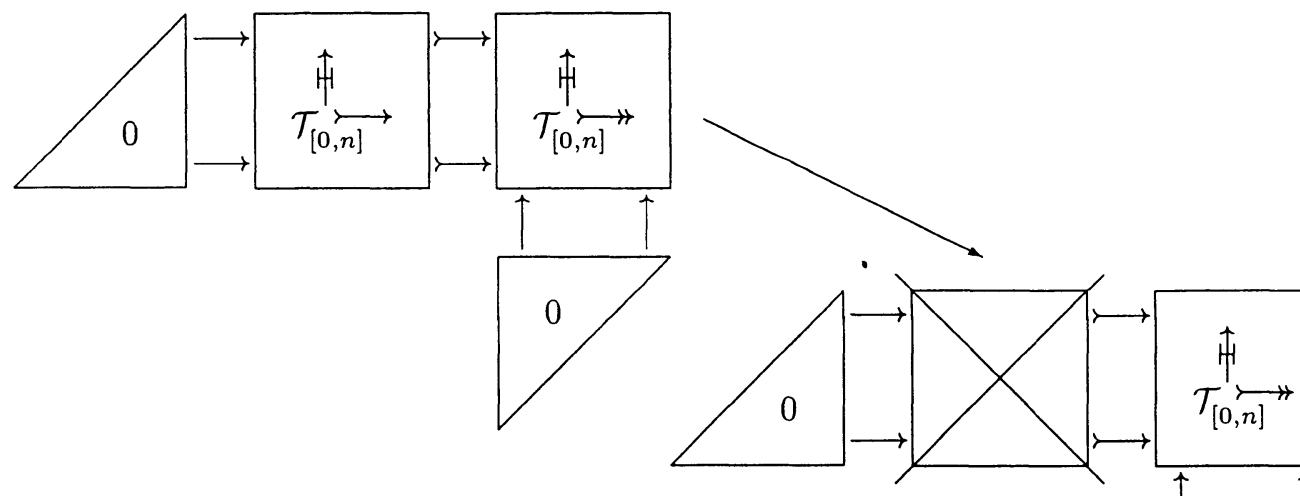

induces a homotopy equivalence. The fiber is

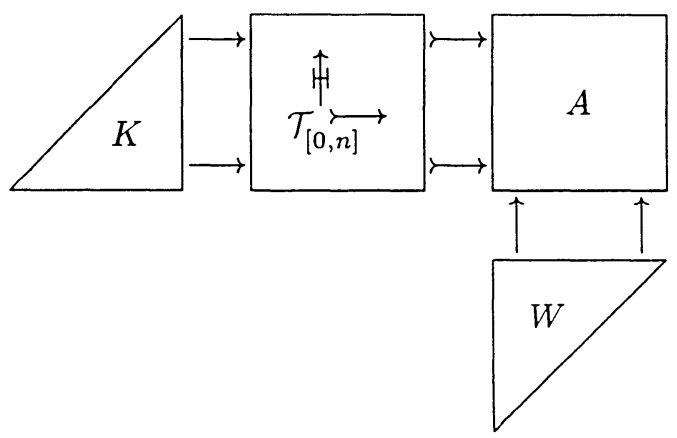

and is contracted by the homotopy 


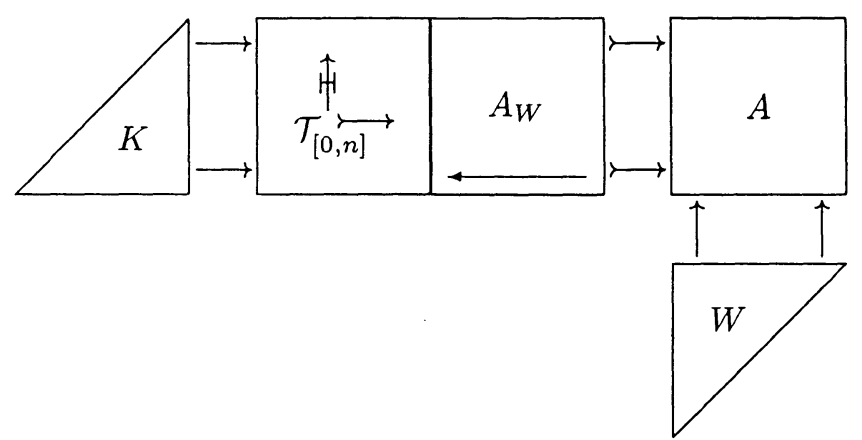

Thus, we are reduced to showing that the natural map

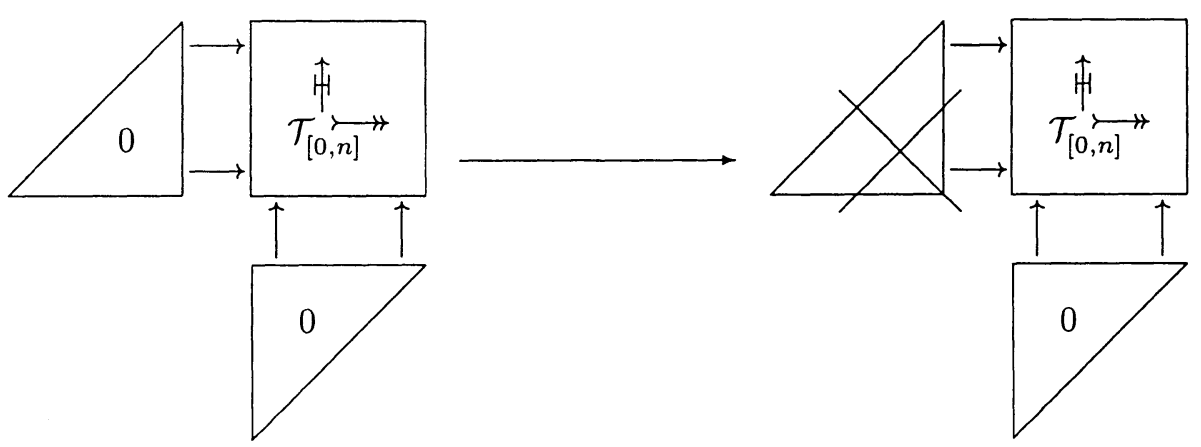

induces a homotopy equivalence. The proof now becomes very similar to the argument we have just seen in Lemma 3.5.3.1. We look at the diagram 


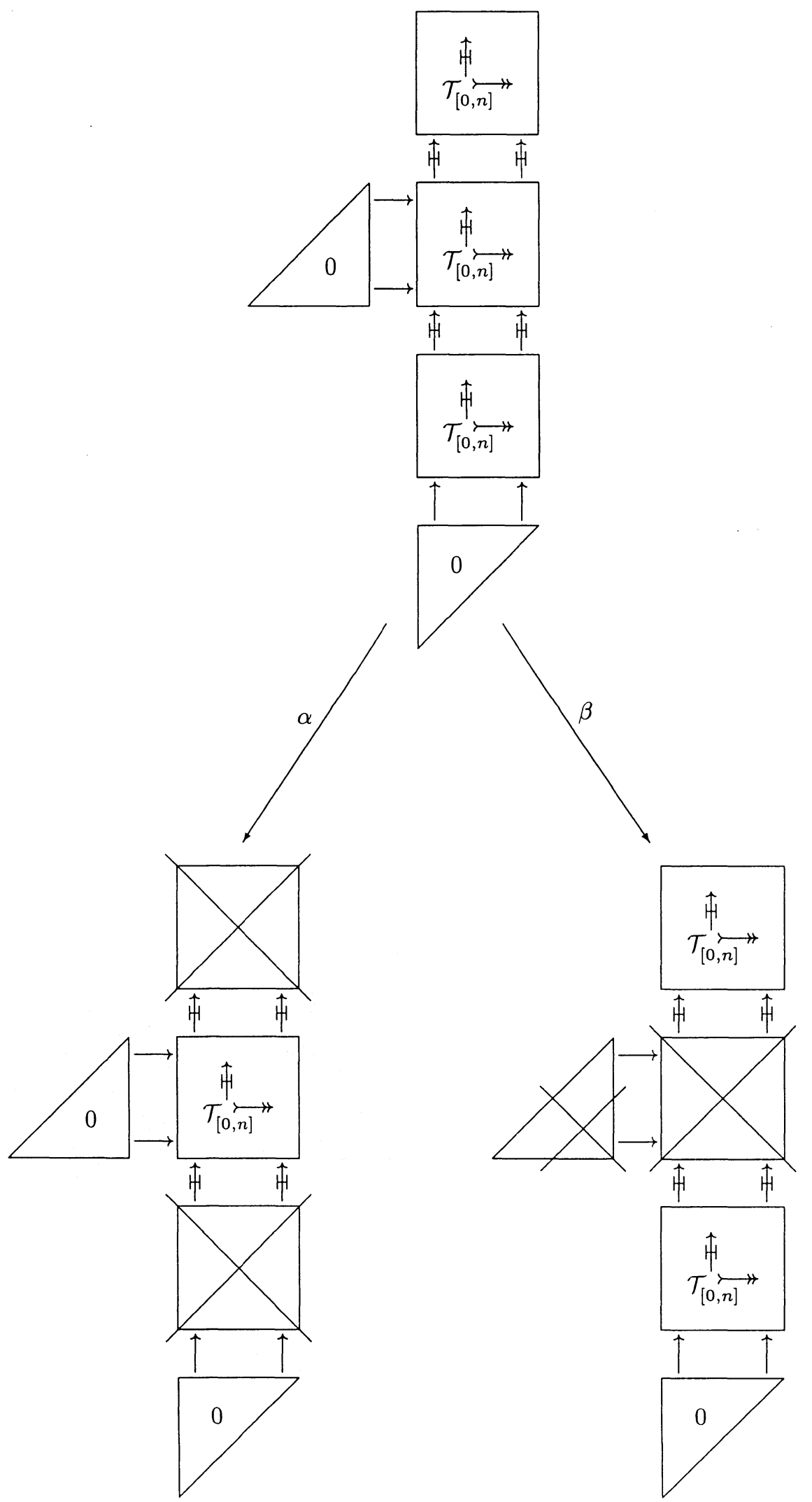


The fact that $\alpha$ induces a homotopy equivalence is again essentially trivial. The Segal fiber is the simplicial set

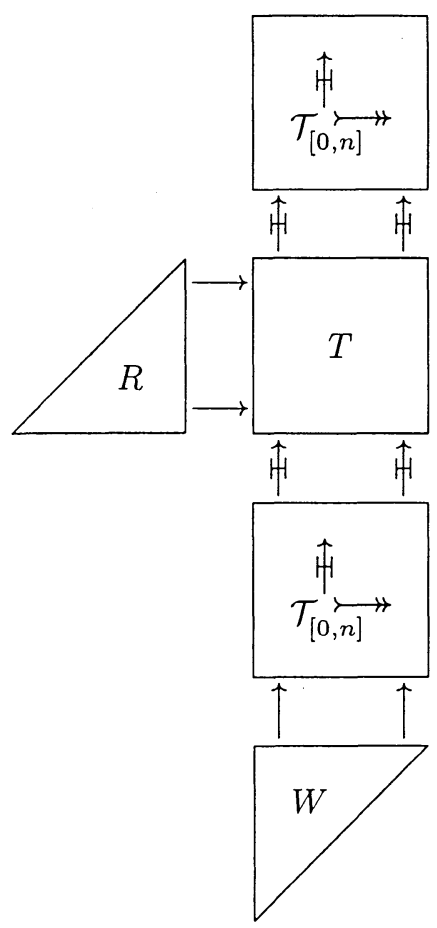




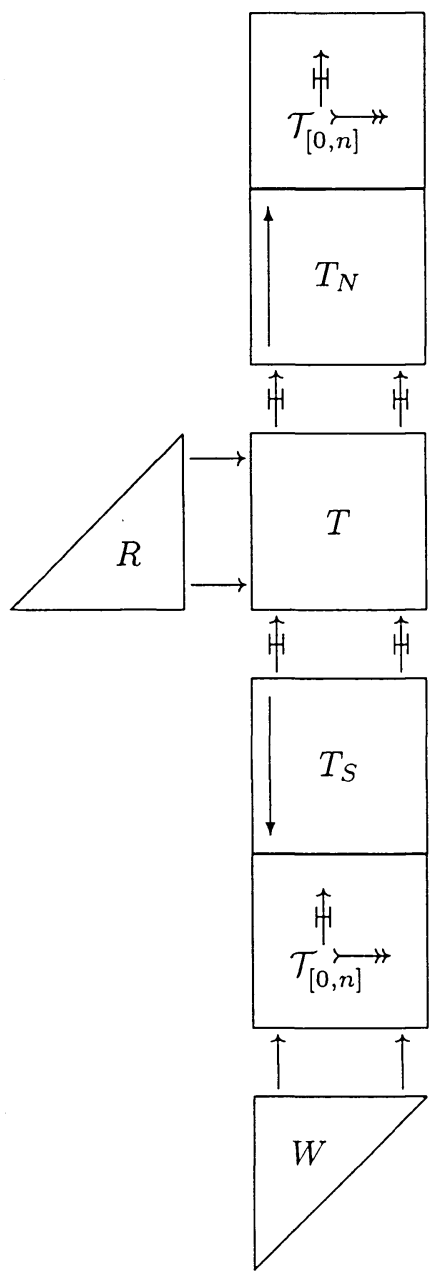

The fact that $\beta$ induces a homotopy equivalence is again a little more subtle. The Segal fiber is the simplicial set 


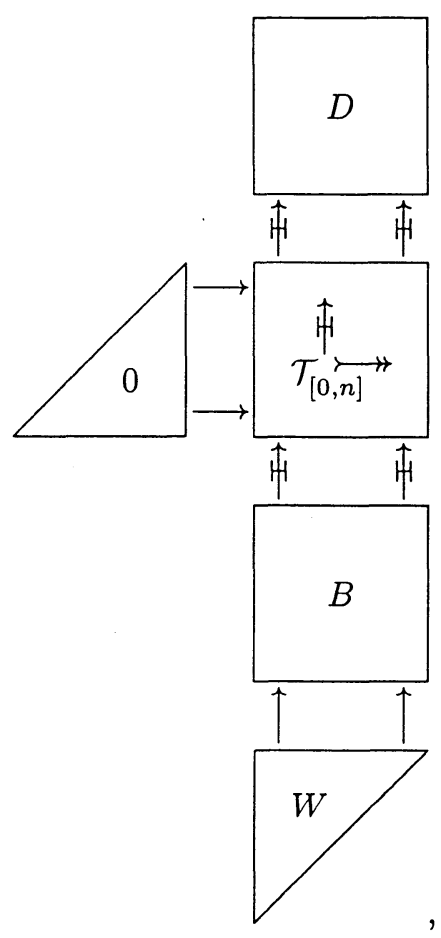

and the point of the proof is that the homotopy

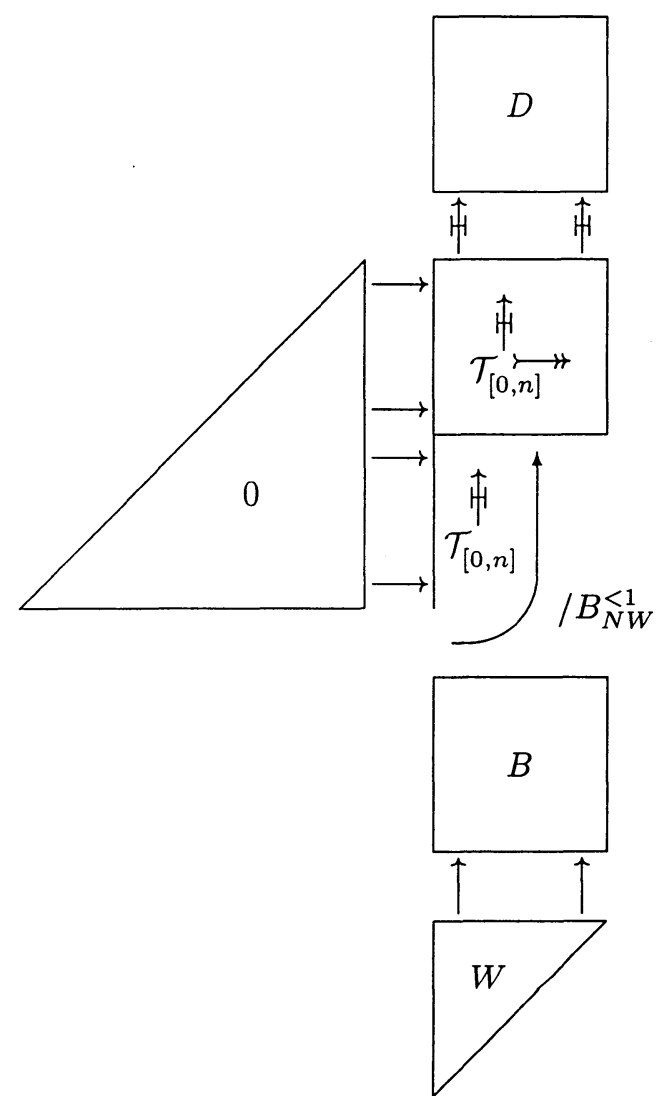


shows that, up to homotopy, the identity on the simplicial set

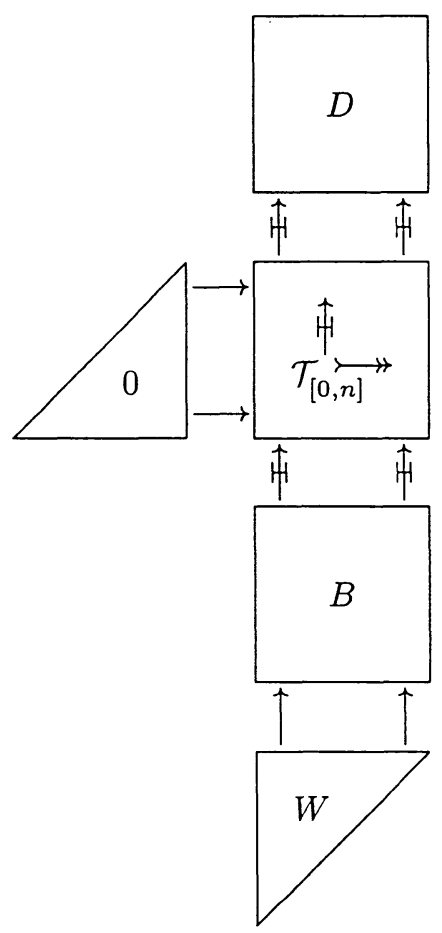

factors through the simplicial set

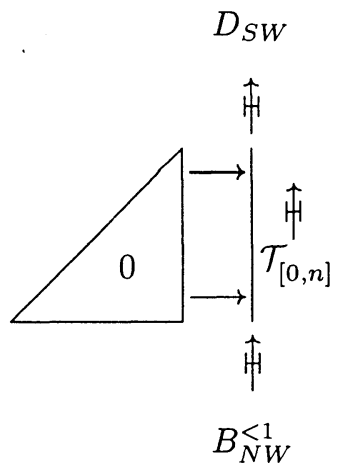

and this last simplicial set is contracted by the contraction to the initial object $B_{N W}^{<1}$. This completes the proof of Lemma 3.5.3.2, and hence also the proof of (3.5.3).

The proof of Lemma 3.5 has been long and tortuous enough, I am sure we have all had time to forget the point of the exercise. It is therefore time for another gathering of the general staff, to recall our battle strategy.

Strategic Reminder. We have been studying the diagram 


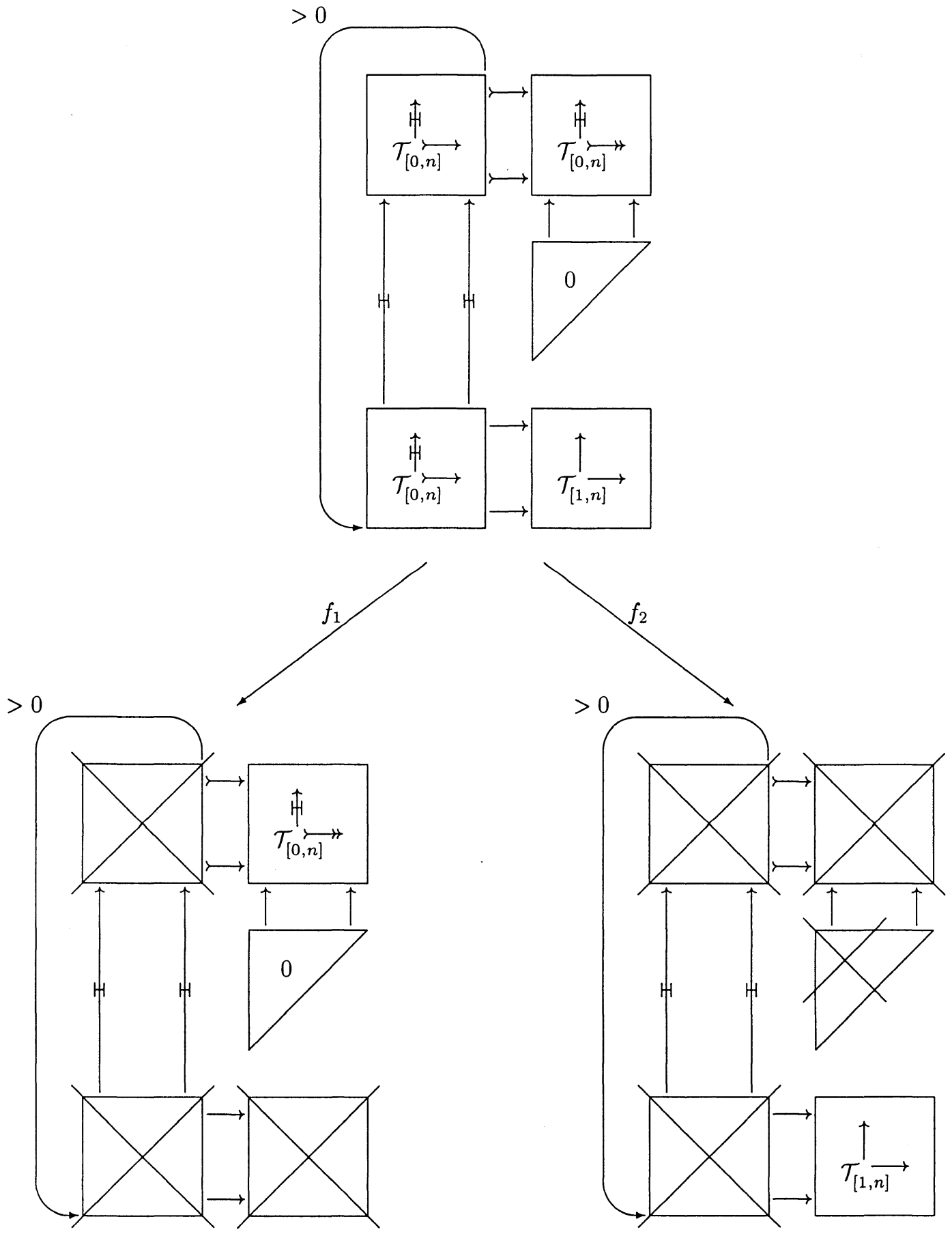

and the point of the somewhat elaborate antics we have just performed was to show that $f_{1}$ induces a homotopy equivalence (Lemma 3.5). The next lemma shows that $f_{2}$ is a quasifibration, and the two lemmas following will identify the homotopy fiber of $f_{2}$ with the simplicial set 


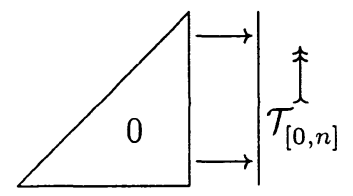

By Section I.7 we know that the contractibility of this simplicial set suffices to prove Theorem I.7.1.

End of Strategic Reminder.

Lemma 3.6. The map $f_{2}$ :
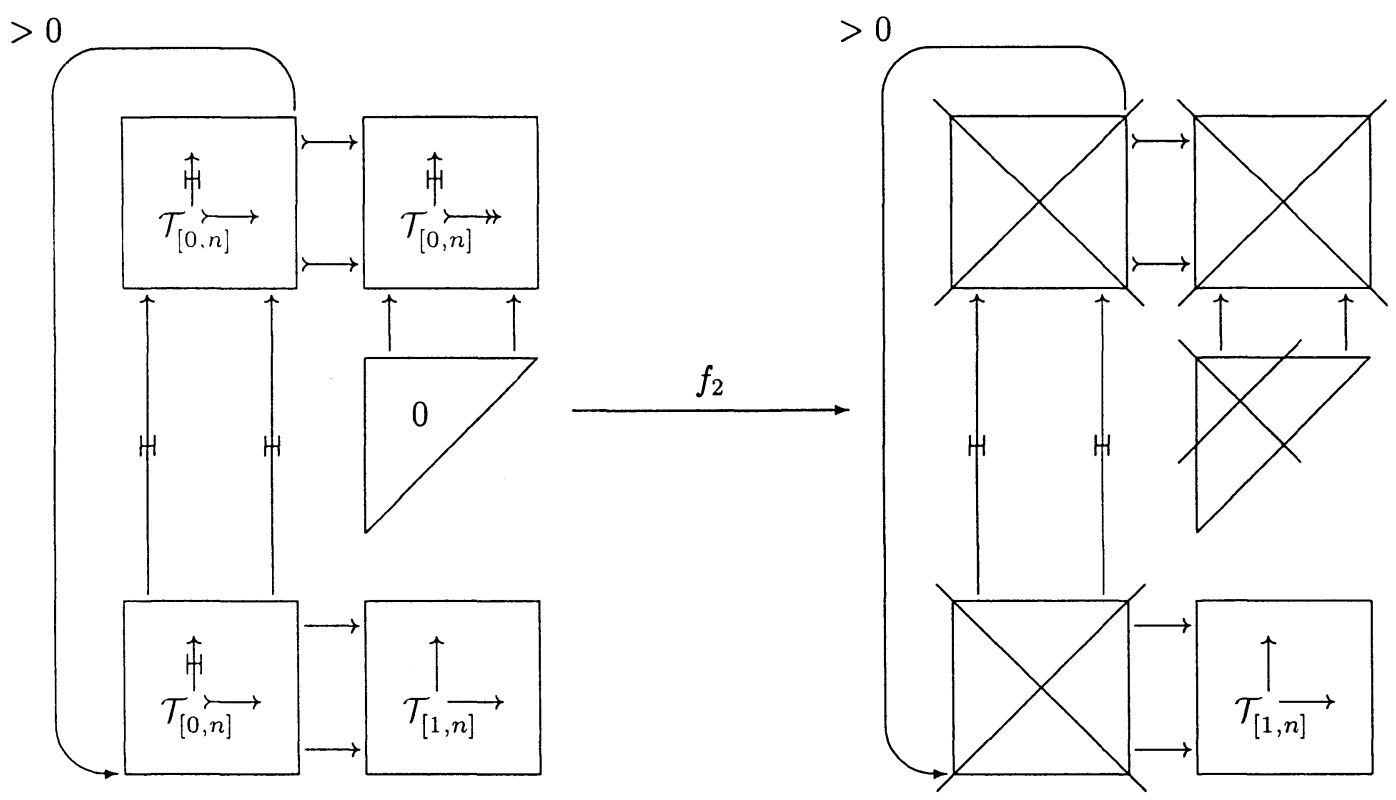

is a quasi-fibration.

Proof. This proof essentially is a standard example of a Prototype Quasifibration 1.2. For that reason, we will be brief.

We must study the fiber 


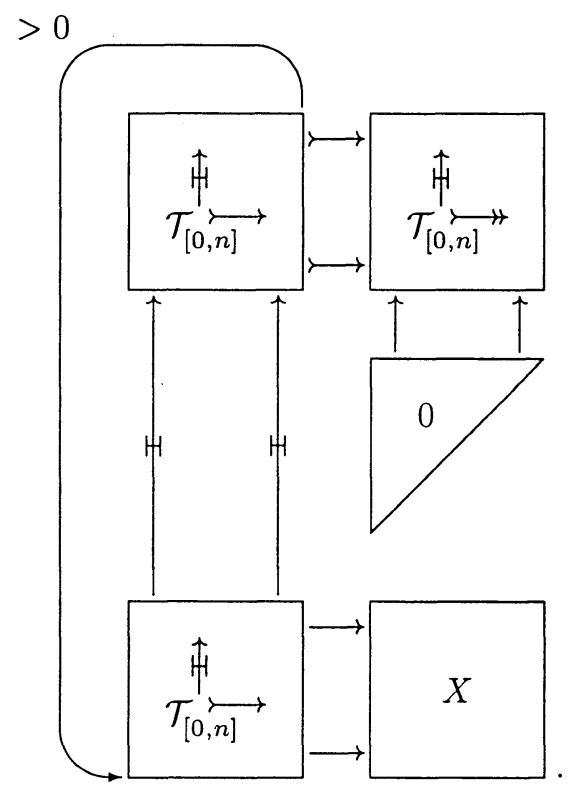

We use the following sequence of homotopies, with the notation as in Section 1:

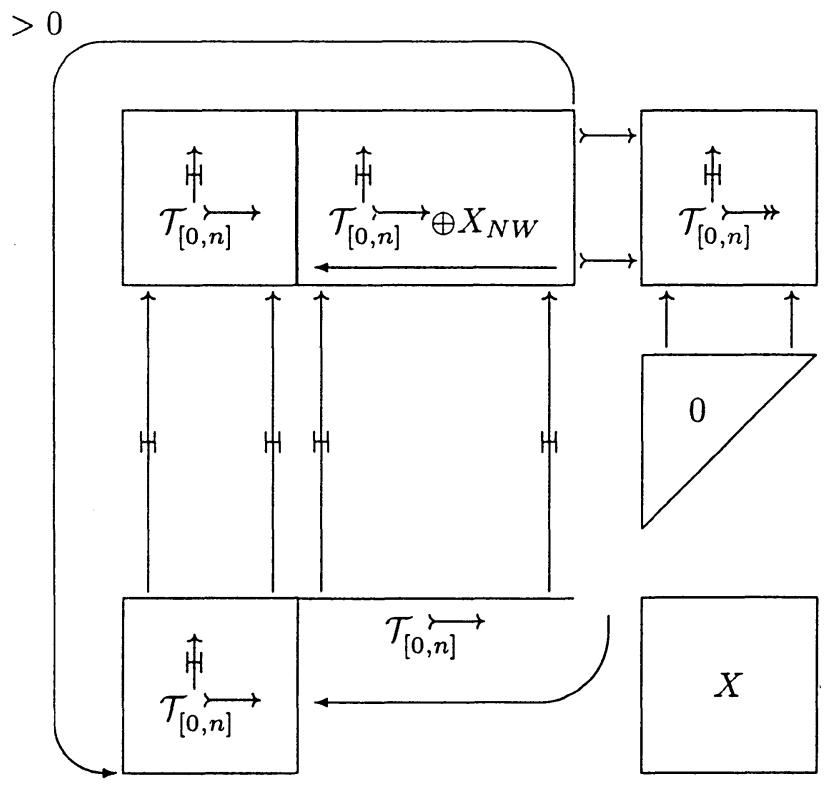




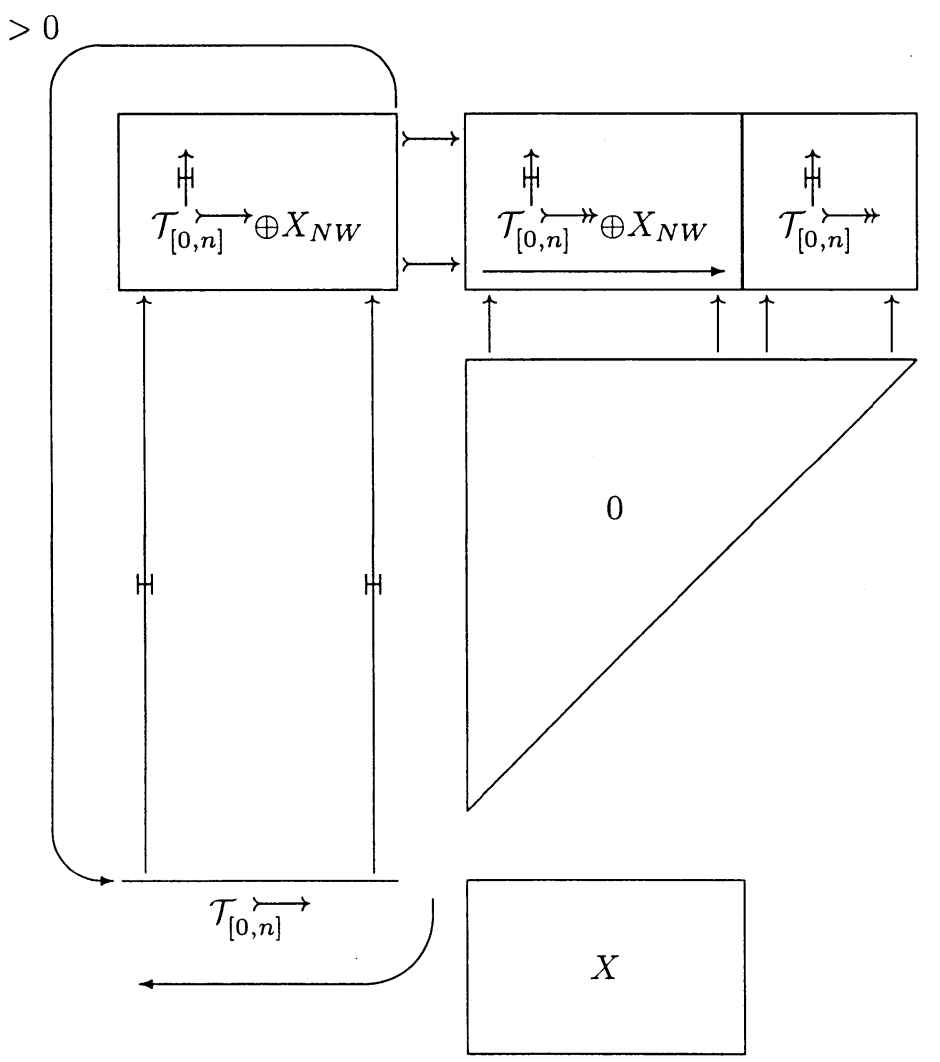

(3.4) 


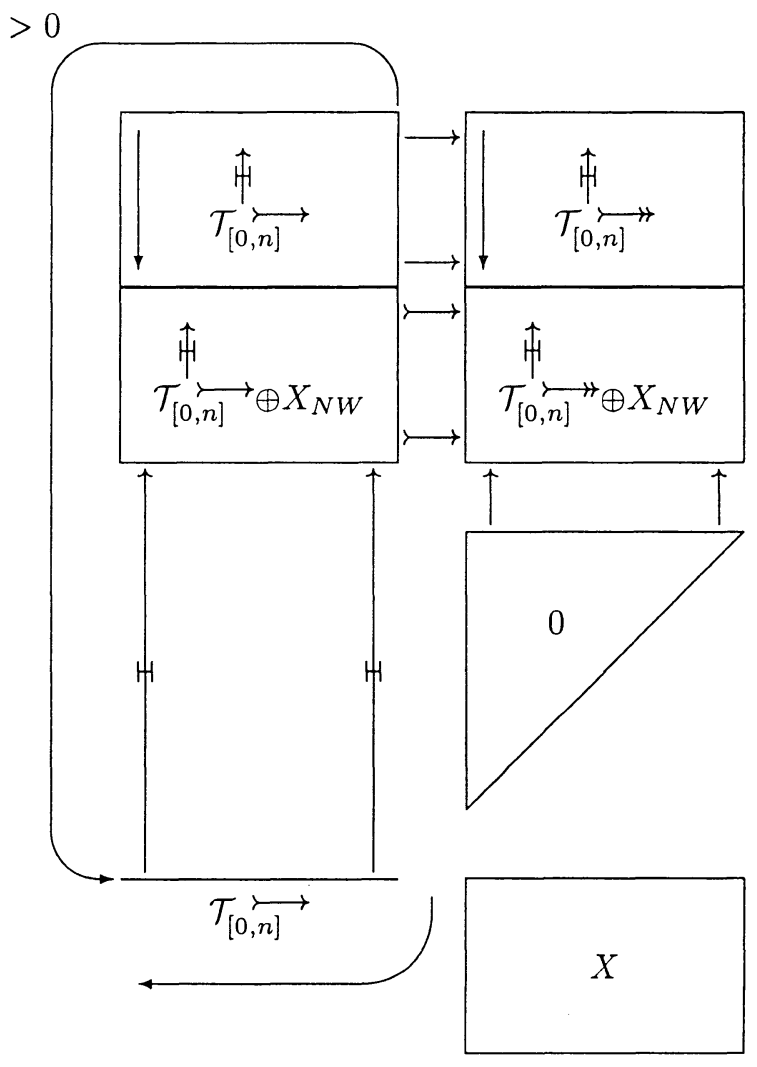

$(3.5)$

These three homotopies combine to give a homotopy of the identity with the map

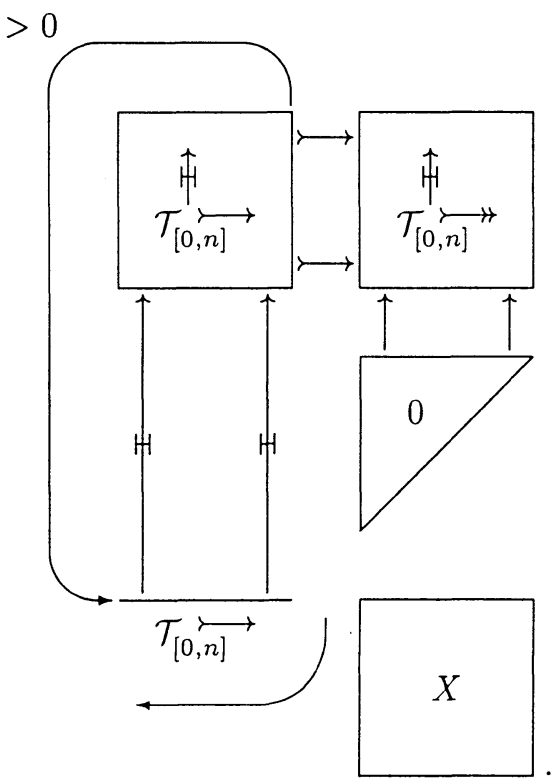

This last map factors as a composite $\psi \circ \phi$, for $\phi$ and $\psi$ as below 


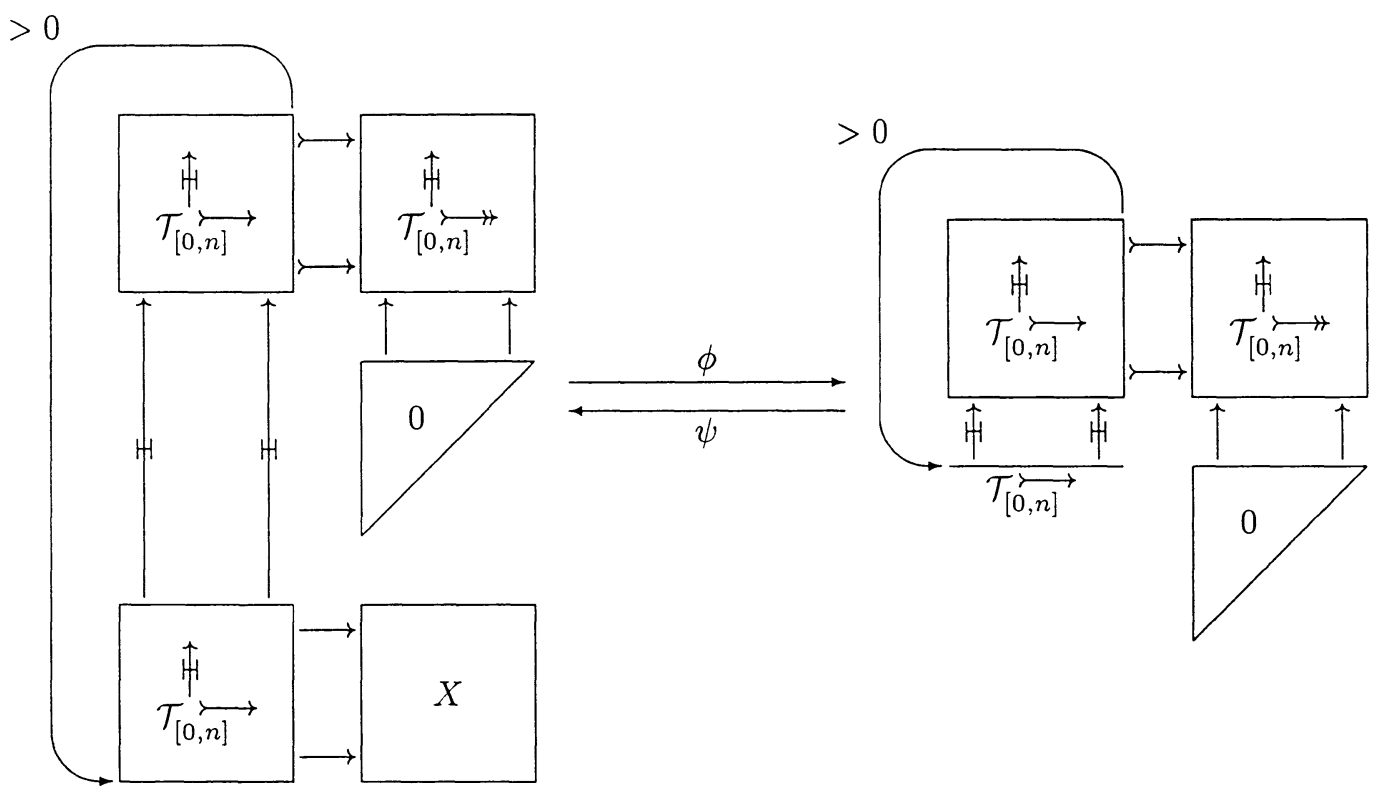

Thus $\psi \circ \phi$ is homotopic to the identity. On the other hand $\phi \circ \psi$ is translation in the $H$-space structure with respect to the zero cell

But the simplicial set $>0$
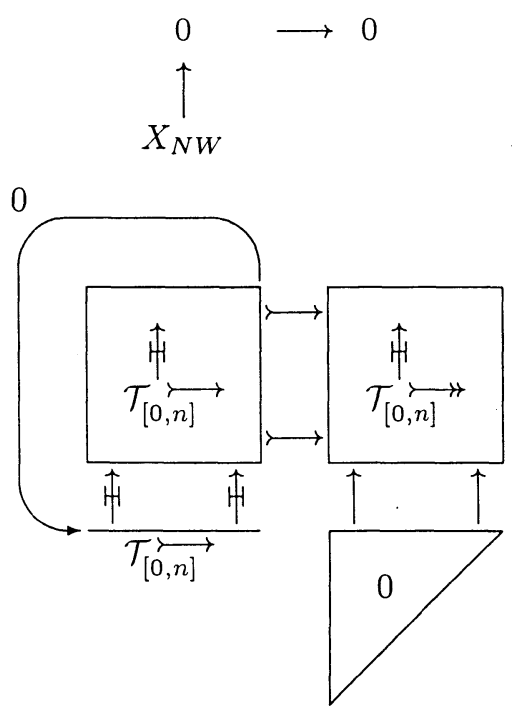

is easily seen to be connected. Even more easily one can see that

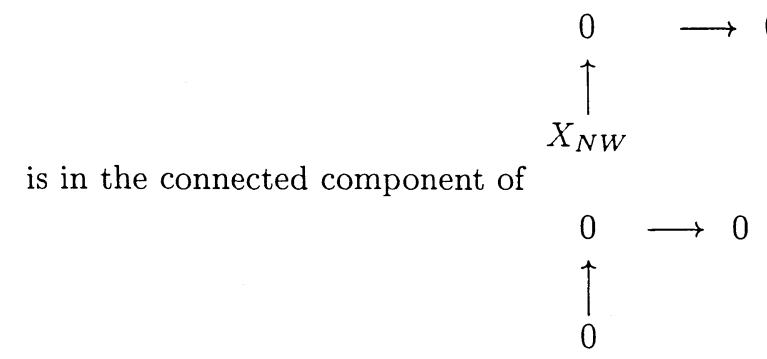


In any case, by any argument that pleases the reader, $\phi$ and $\psi$ are homotopy equivalences.

Similarly, if $\partial$ is any face map, then $\psi \circ \partial \circ \phi$ is translation in the $H$-space structure with respect to

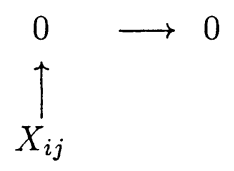

also a 0 -cell which is clearly in the identity component. Thus $\partial$ is a homotopy equivalence, and we are done.

LEMma 3.7. There is a natural map

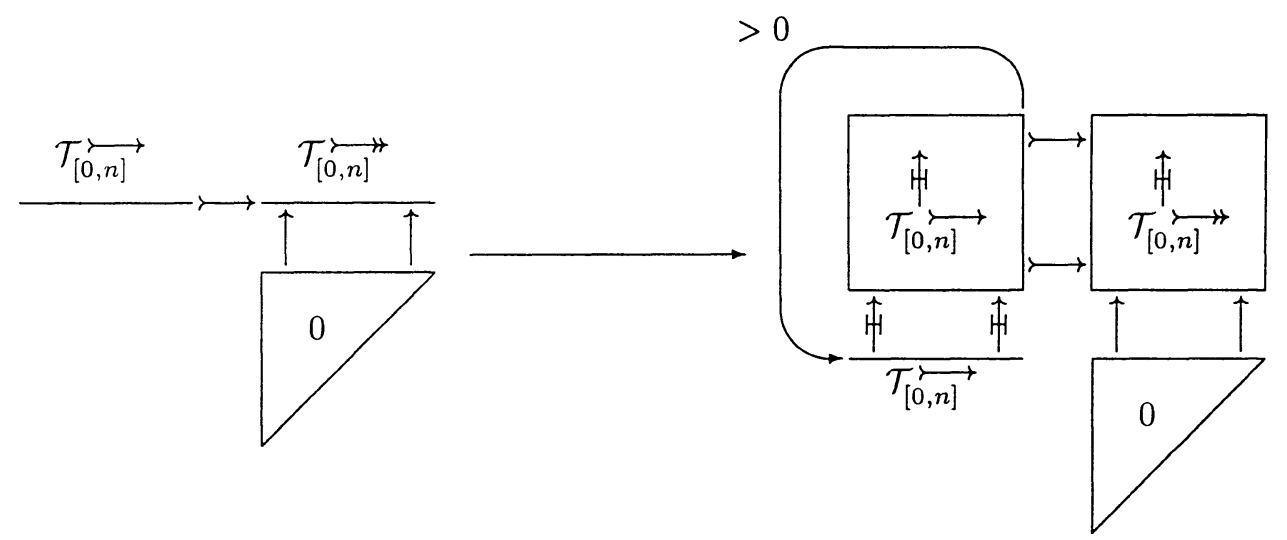

which induces a homotopy equivalence. The map take the simplex

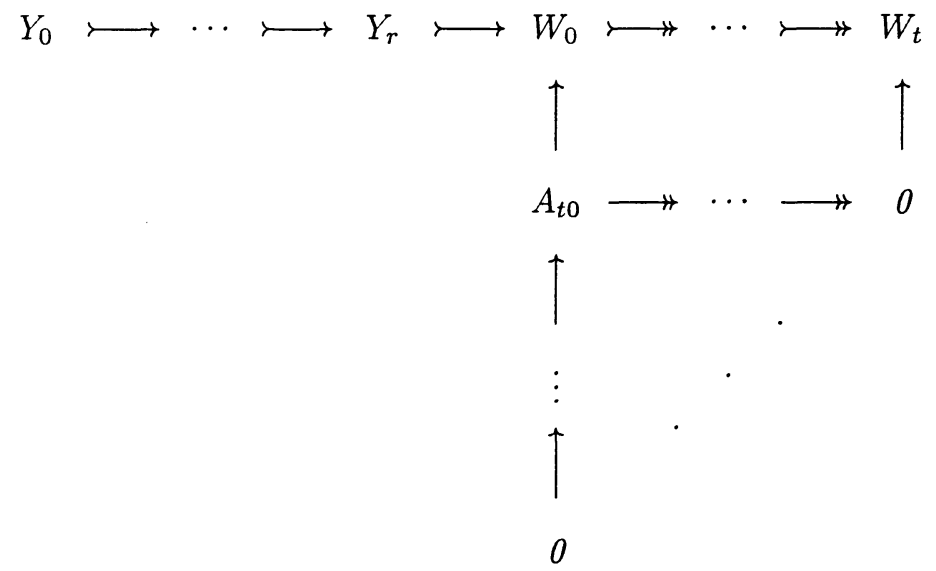

to the simplex 


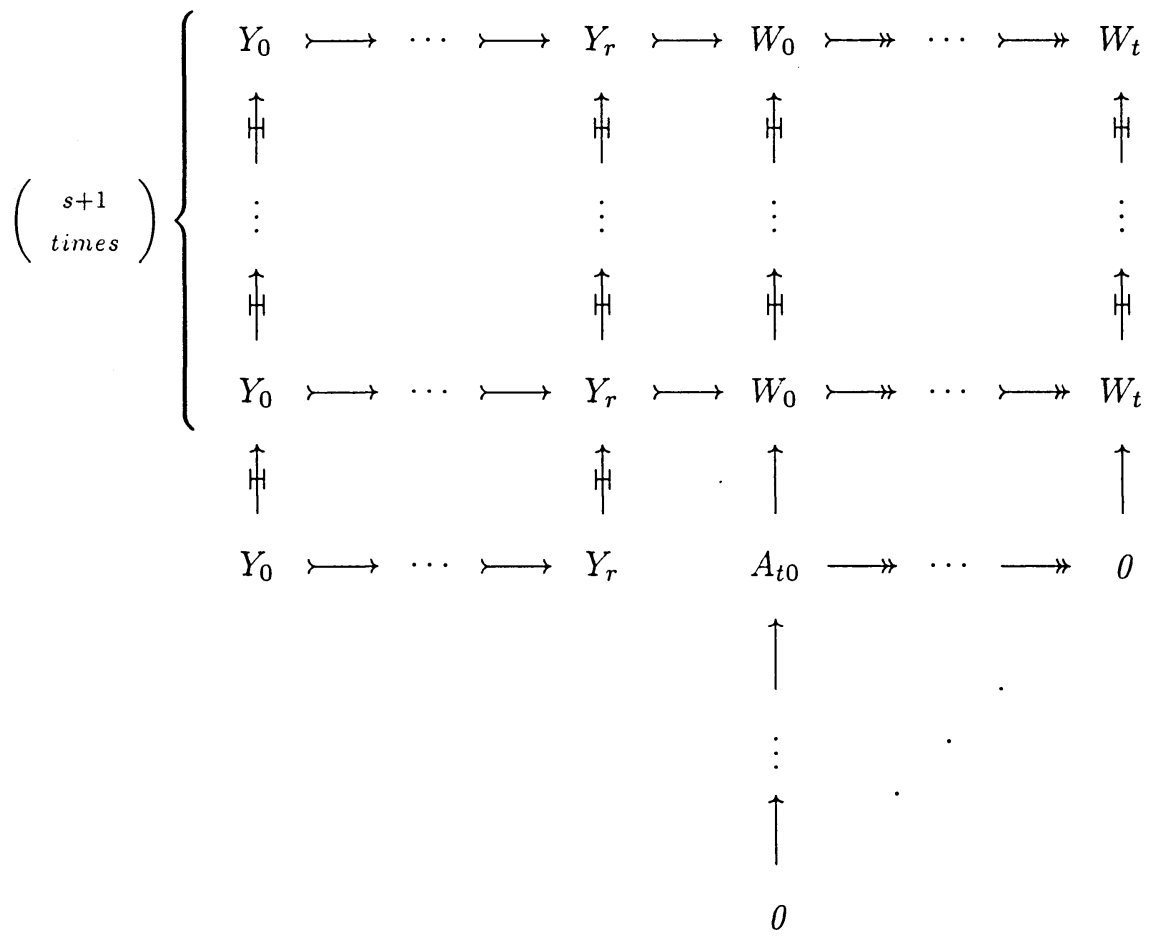

Proof. It suffices, by Segal's theorem, to prove that when we realize the simplicial structures corresponding to $s$ and $t$, the resulting map of simplicial spaces is a homotopy equivalence. The resulting simplicial spaces have for their $r$-simplices the disjoint union of the realizations of

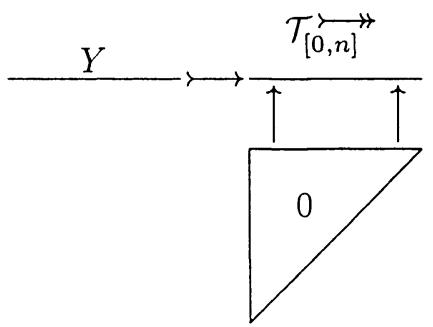

respectively 


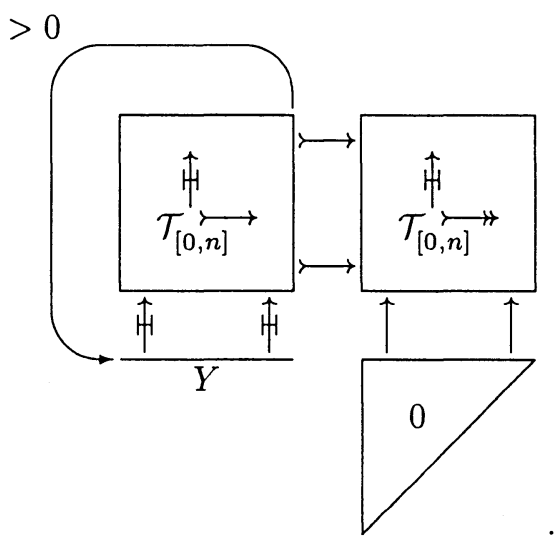

over all $Y=\left(Y_{0} \longmapsto \cdots \longmapsto Y_{r}\right)$. Thus, it will suffice to prove that, for fixed $Y$, the natural map is a homotopy equivalence.

By our favorite homotopy, there is a homotopy equivalence

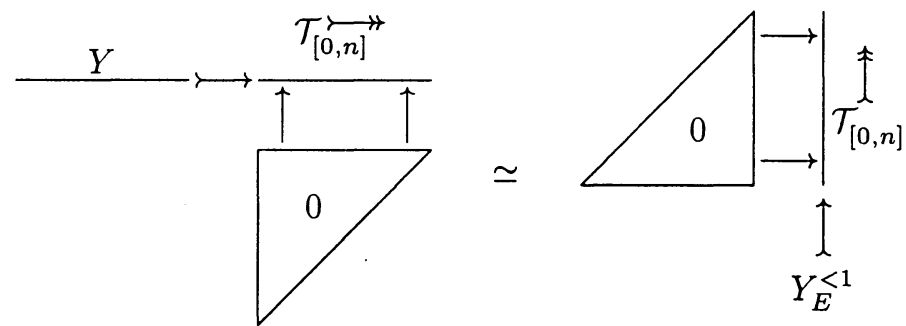

Similarly, there is a homotopy equivalence

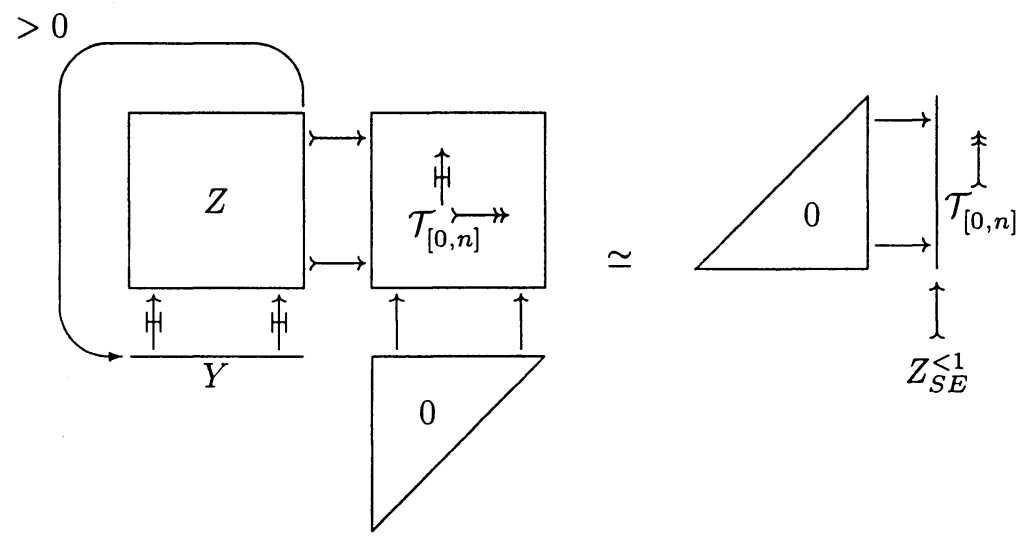

(We remind the reader that in both cases one uses the homotopy 
$>0$

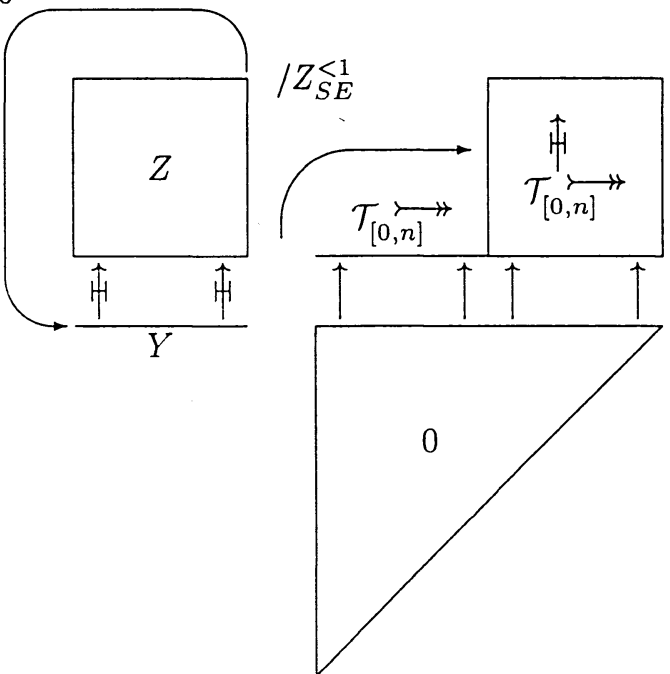

This homotopy is a little hard to draw when the simplices are 1-dimensional. Recall now the compatibility requirement on the truncated differentials, which guarantees that the end map of the homotopy indeed factors through

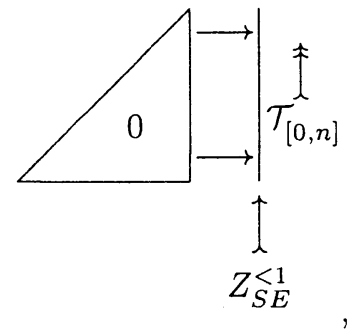

as we said.)

Let us now look a little more closely at the homotopy equivalence (3.7). It identifies for us the homotopy type of the Segal fiber of the projection

$>0$

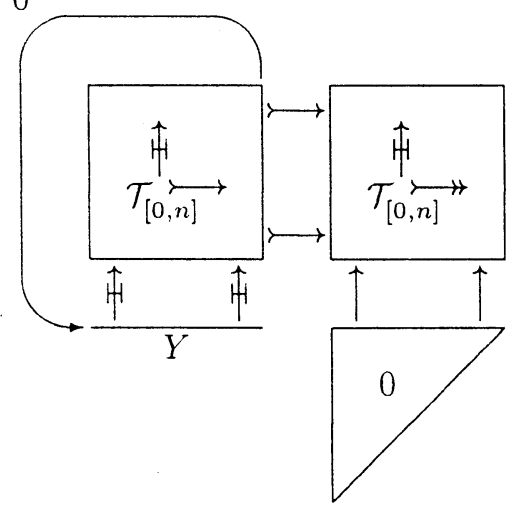

and the Segal fiber, being

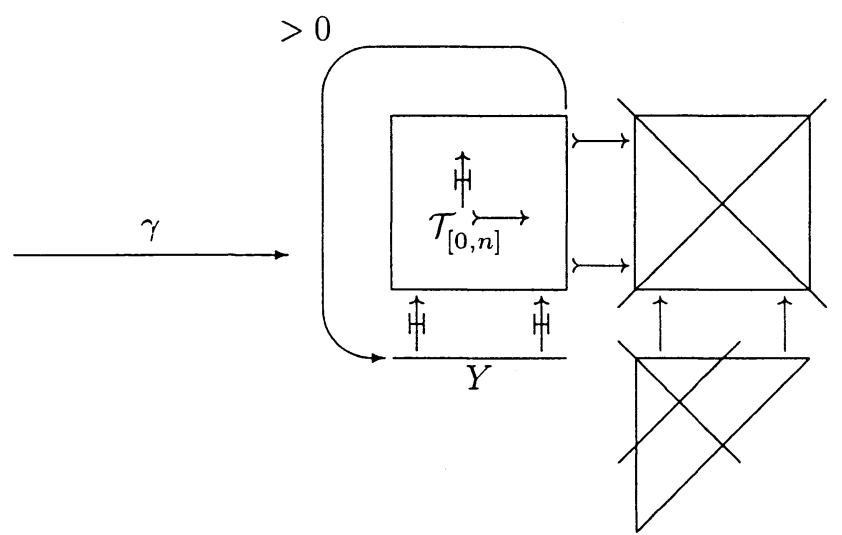




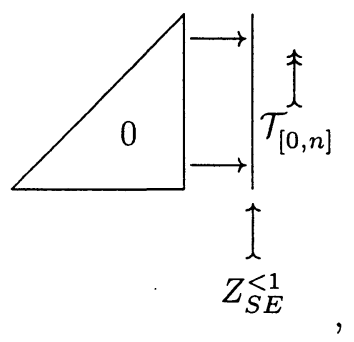

has a homotopy type independent of $Z$. After all, $Y_{E}^{<1}=Z_{S E}^{<1}$. This is because the map $Y_{E} \longrightarrow Z_{S E}$ is by assumption an $H^{0}$-isomorphism.

It follows that all the fibers of $\gamma$ are homotopy equivalent, and the reader can easily check that in fact $\gamma$ is a quasifibration. But the target of $\gamma$ is a contractible space. We deduce a homotopy equivalence
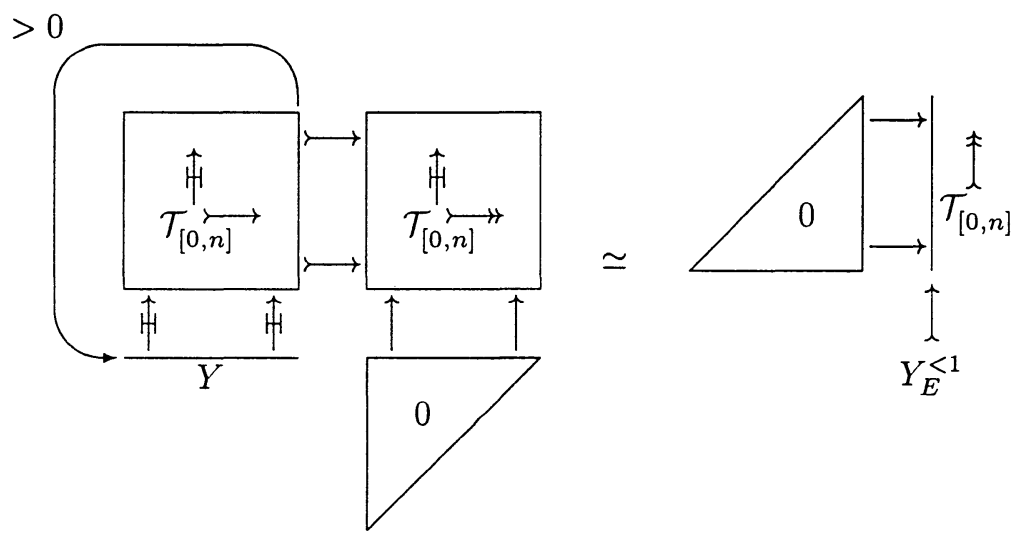

Comparing the homotopy equivalences (3.6) and (3.8), Lemma 3.7 follows immediately.

LEMMA 3.8. The projection
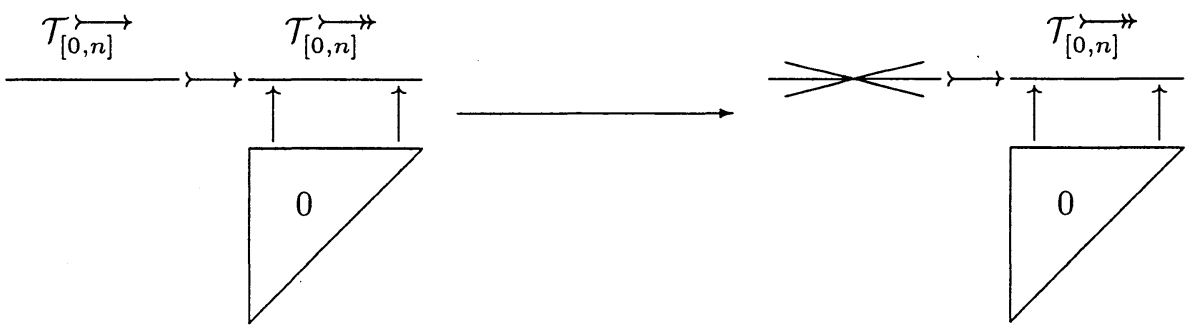

is a homotopy equivalence.

Proof. Trivial. The Segal fiber is the nerve of a category, and the reader gets his choice whether to contract it to the initial or the terminal object.

Conclusions from Lemmas 3.5-3.8. The natural truncation map 

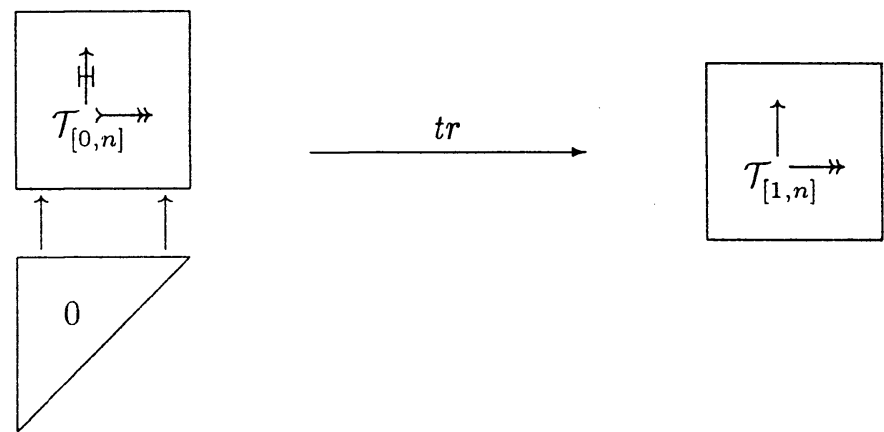

has for its homotopy fiber the simplicial set

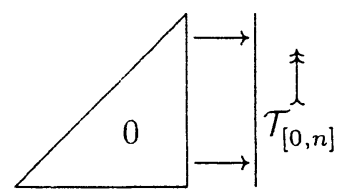

What has actually been proved is a little less. We proved that there is a map
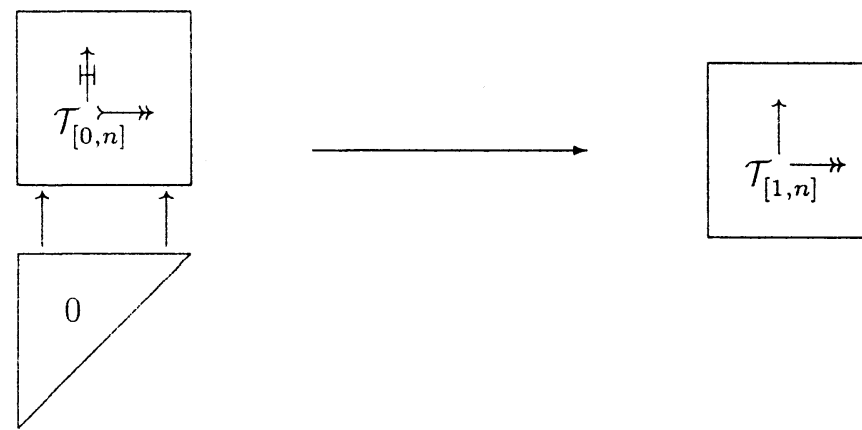

having the homotopy fiber

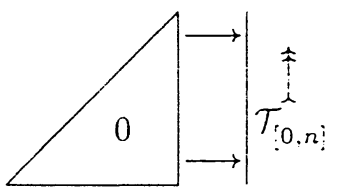

But we have not proved that the map is induced by the truncation. Let us be uncharacteristically honest and complete about this argument. What we have really proved is that in the diagram 


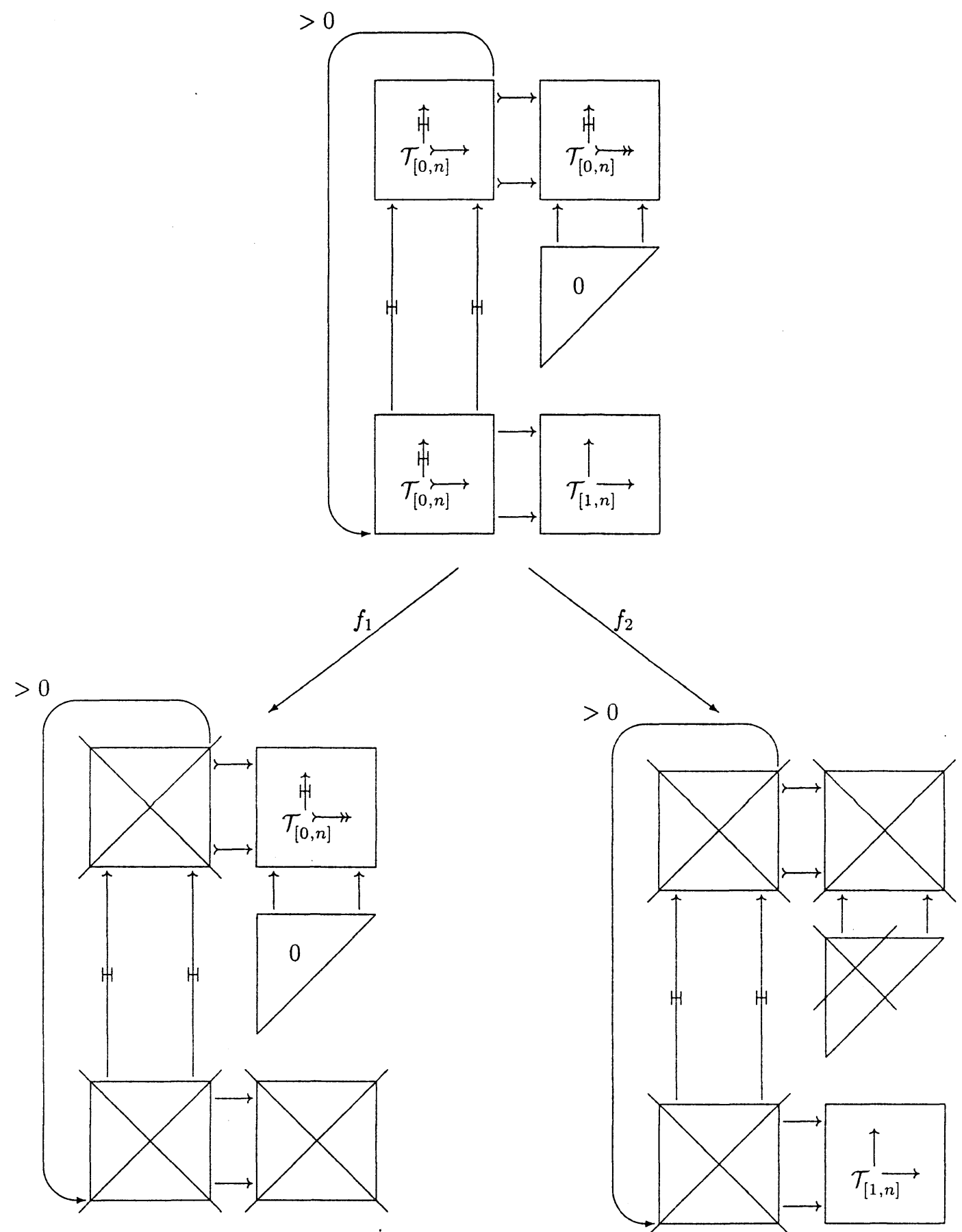

the map $f_{1}$ is a homotopy equivalence (Lemma 3.5), and the map $f_{2}$ is a quasifibration with fiber

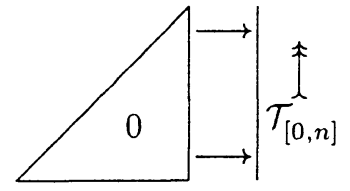


But in fact, the map $f_{2}$ is split. There is a map
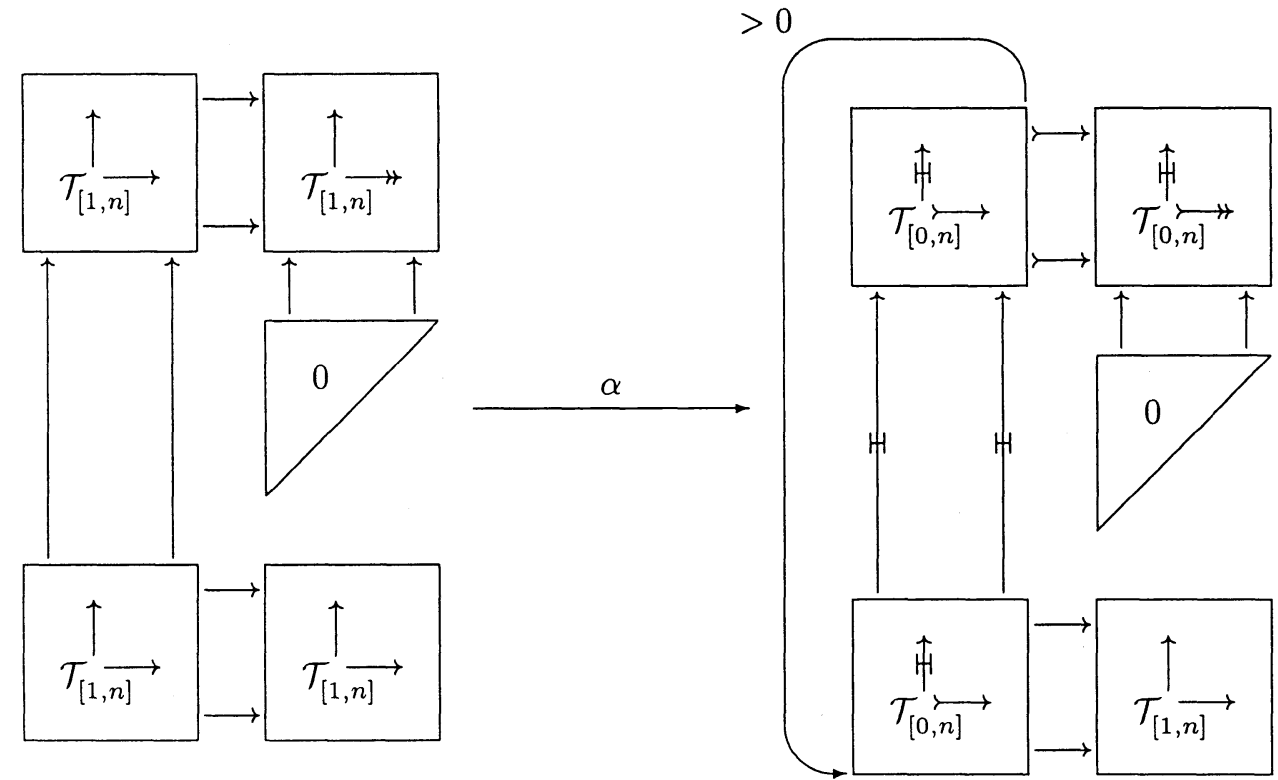

and it is completely clear that $f_{2} \circ \alpha$ is a homotopy equivalence. So at least on the level of homotopy groups we get a splitting of

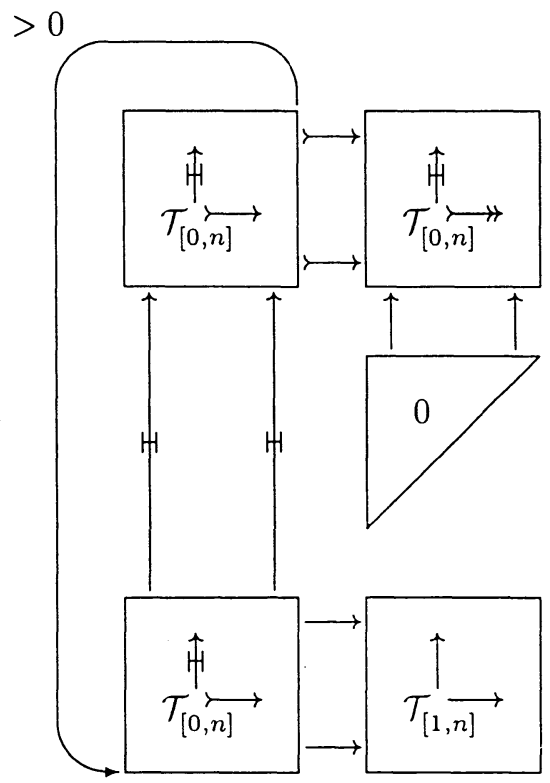

as the direct sum of 


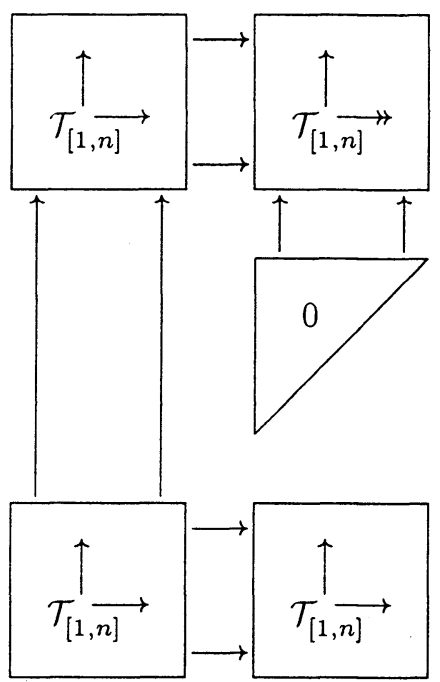

and

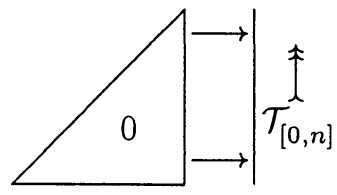

But the composite of $f_{1}$ with the truncation
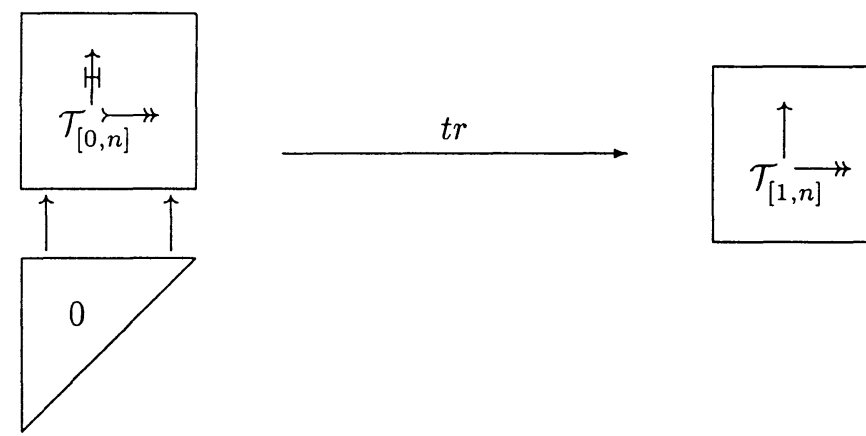

is another splitting for $\alpha$; precisely, the composite $\operatorname{tr} \circ f_{1} \circ \alpha$ is a homotopy equivalence. It is easy to compute that $\operatorname{tr} \circ f_{1}$ vanishes on the fiber

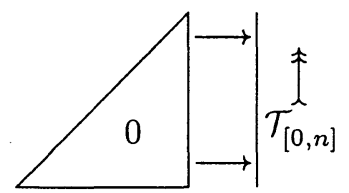

So at least on the level of homotopy groups, the maps $\operatorname{tr} \circ f_{1}$ and $f_{2}$ agree. There is a map from

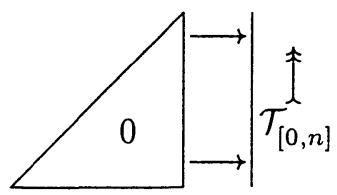

to the homotopy fiber of $\operatorname{tr} \circ f_{1}$, and by what is now an easy homotopy group computation, this map is an isomorphism of homotopy groups, hence a homotopy equivalence.

Note that in the case of $G r$, we achieved as much with far less pain. The many pages of complicated homotopies we have just been through prove the analogue of the miserable little Lemma I.8.1.

Another Gathering of the Strategic Minds. It is time to gather together again, and plan our course of action for the next few lemmas. Until now we have been studying the diagram 
$>0$

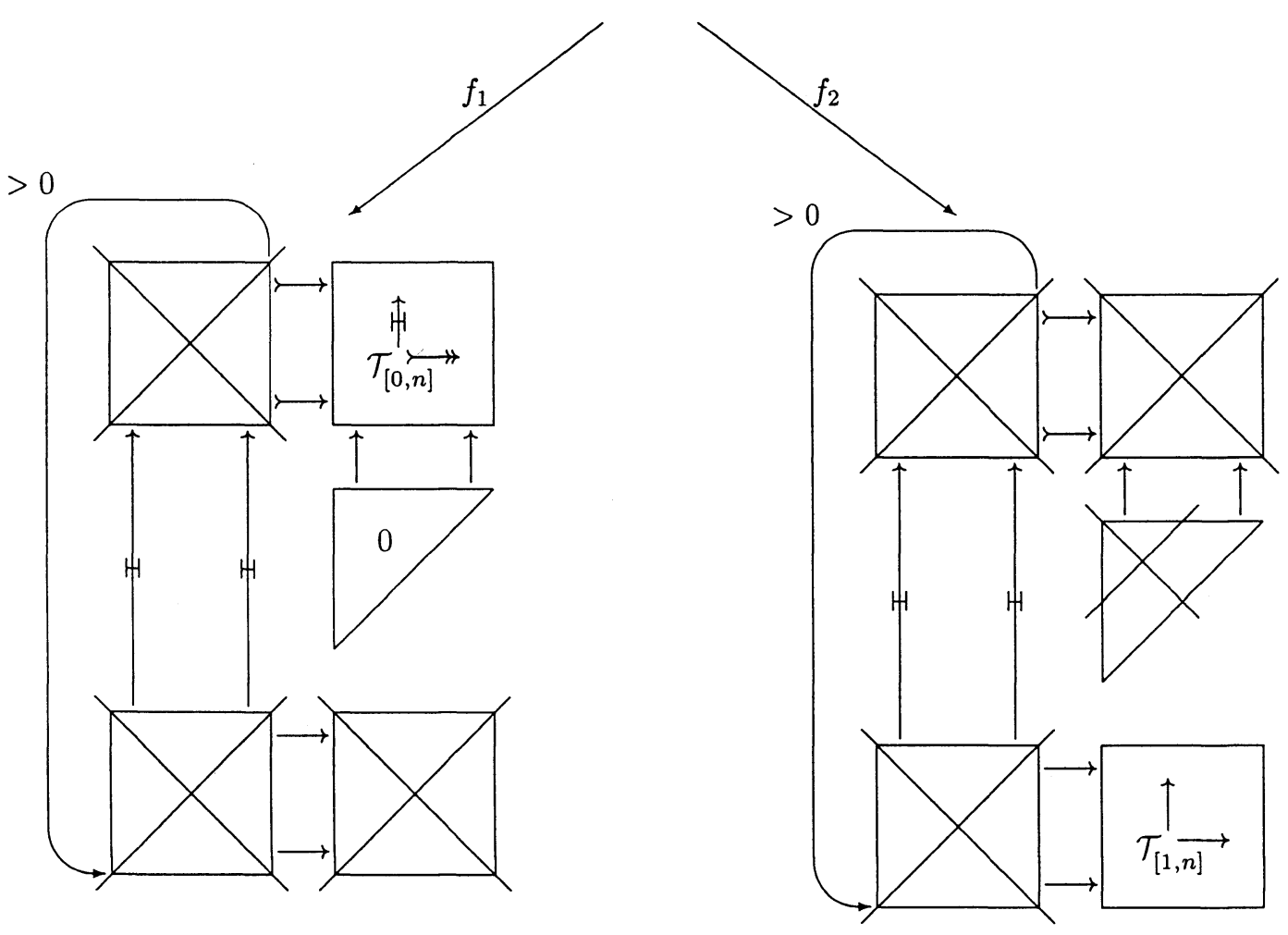

Our project for the next few lemmas is to repeat everything we have done, but this time in the simplicial set with the kernels remembered. Precisely, we will stare at the diagram 


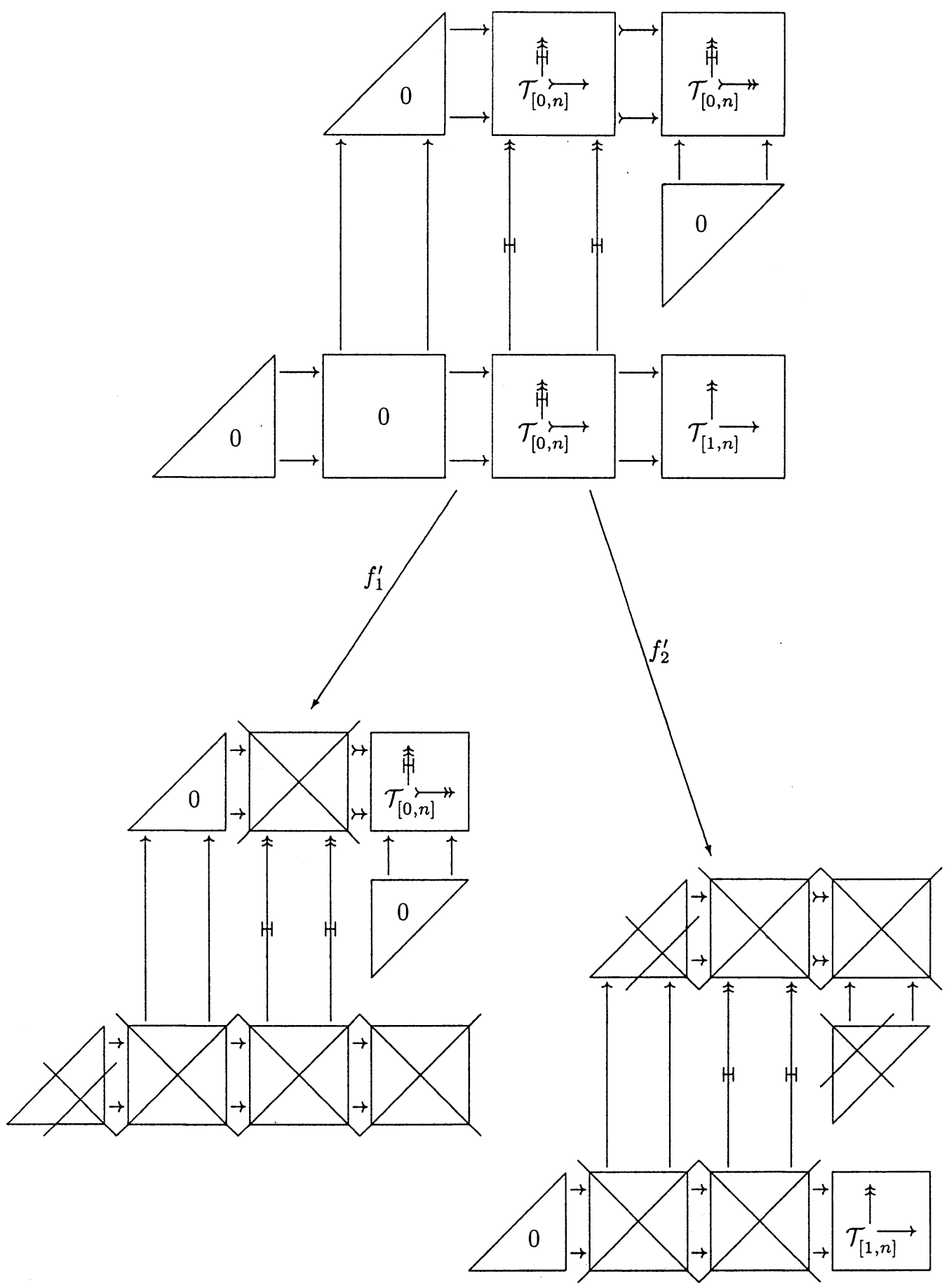

and exactly as before, we will prove that the map $f_{1}^{\prime}$ is a homotopy equivalence, whereas $f_{2}^{\prime}$ is a quasifibration. And then we will also identify the homotopy fiber of $f_{2}^{\prime}$ with the simplicial set 


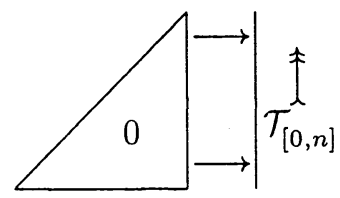

The argument is virtually identical with what we have just gone through, except that the proof that $f_{1}^{\prime}$ induces a homotopy equivalence is much easier. Because the kernels are now part of the structure, we do not have to worry about the truncated differentials; their existence is guaranteed. We will sketch the proof again, just to remind ourselves what has to be done. The reader willing to believe the author can skip this, and go right on to the next strategic gathering.

\section{End of Strategy Session.}

Lemma 3.5'. The simplicial map $f_{1}^{\prime}$

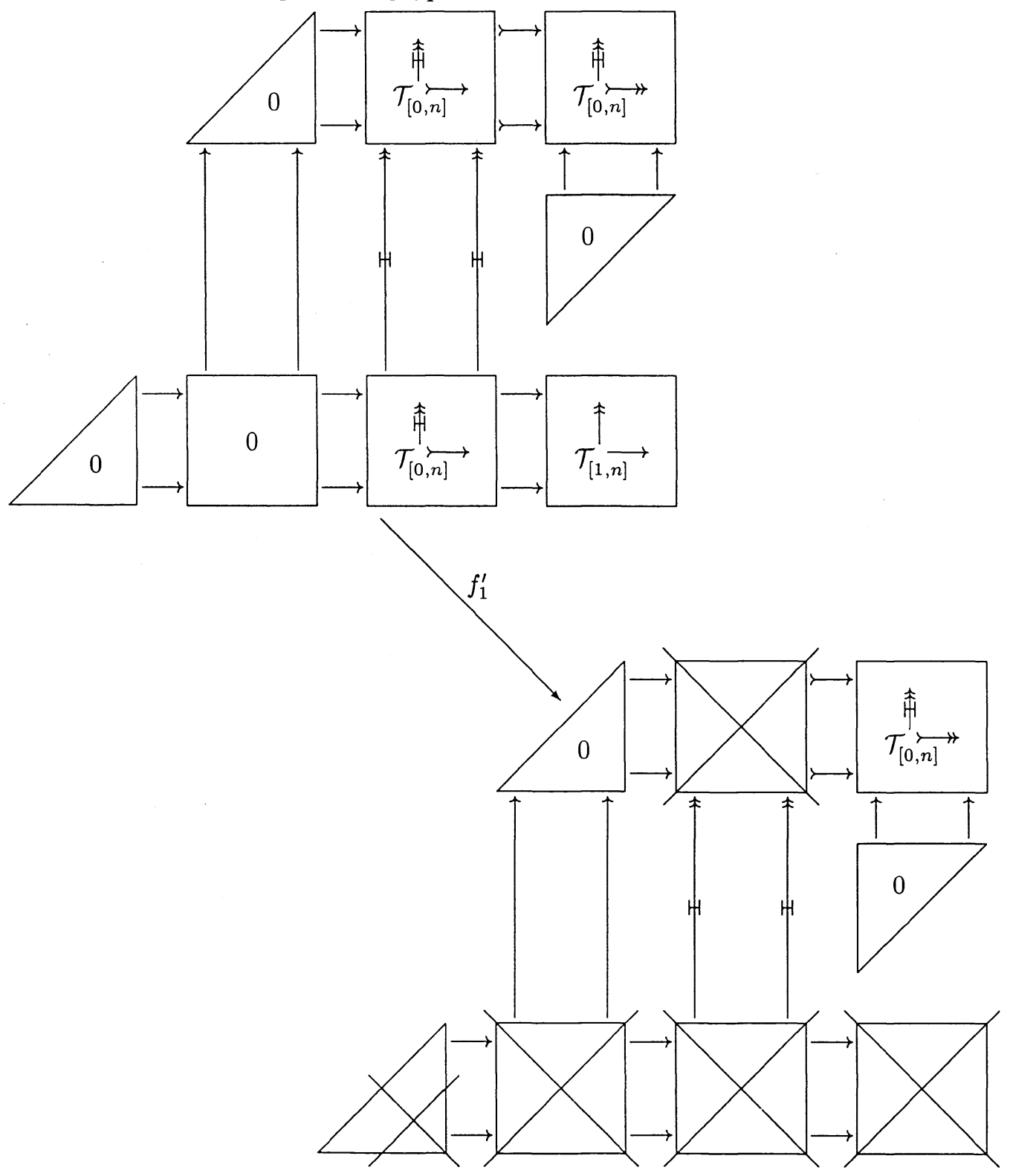


induces a homotopy equivalence.

Proof. Clear.

Lemma 3.6'. The projection

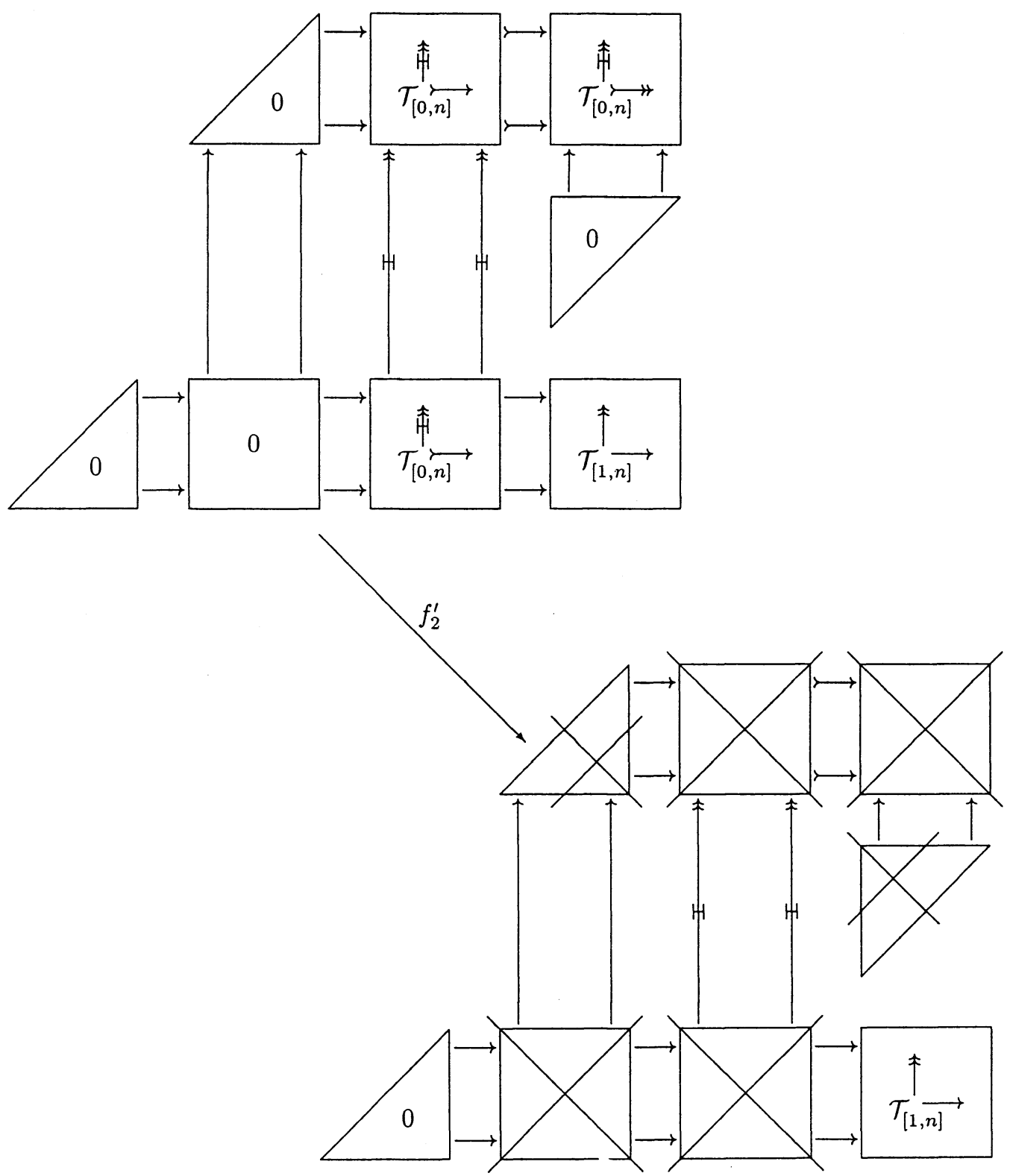

is a quasi-fibration.

Proof. In the language of Section 1, whereas without the kernels this was a Prototype Quasifibration 1.2, now it is Prototype Quasifibration 1.4. 


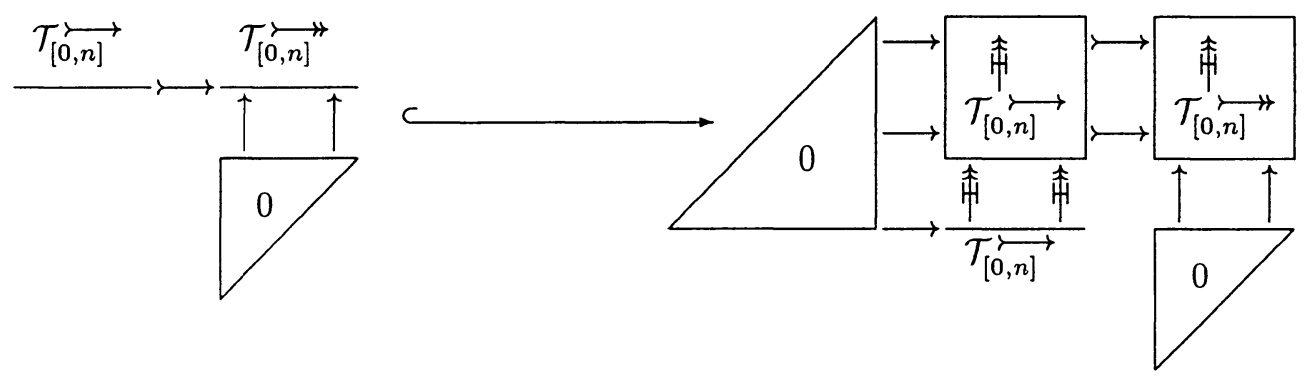

induces a homotopy equivalence. The inclusion in question is the map sending the simplex

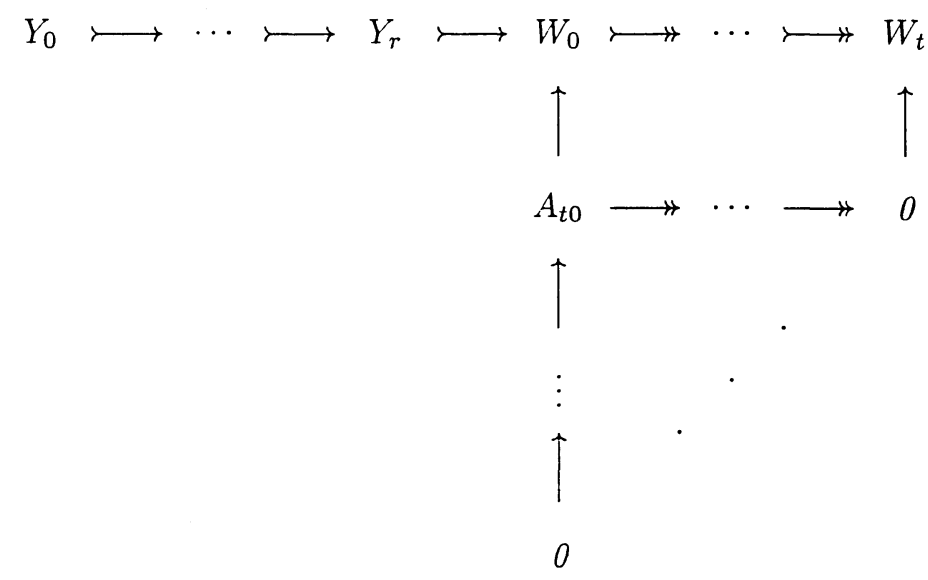

to the simplex 


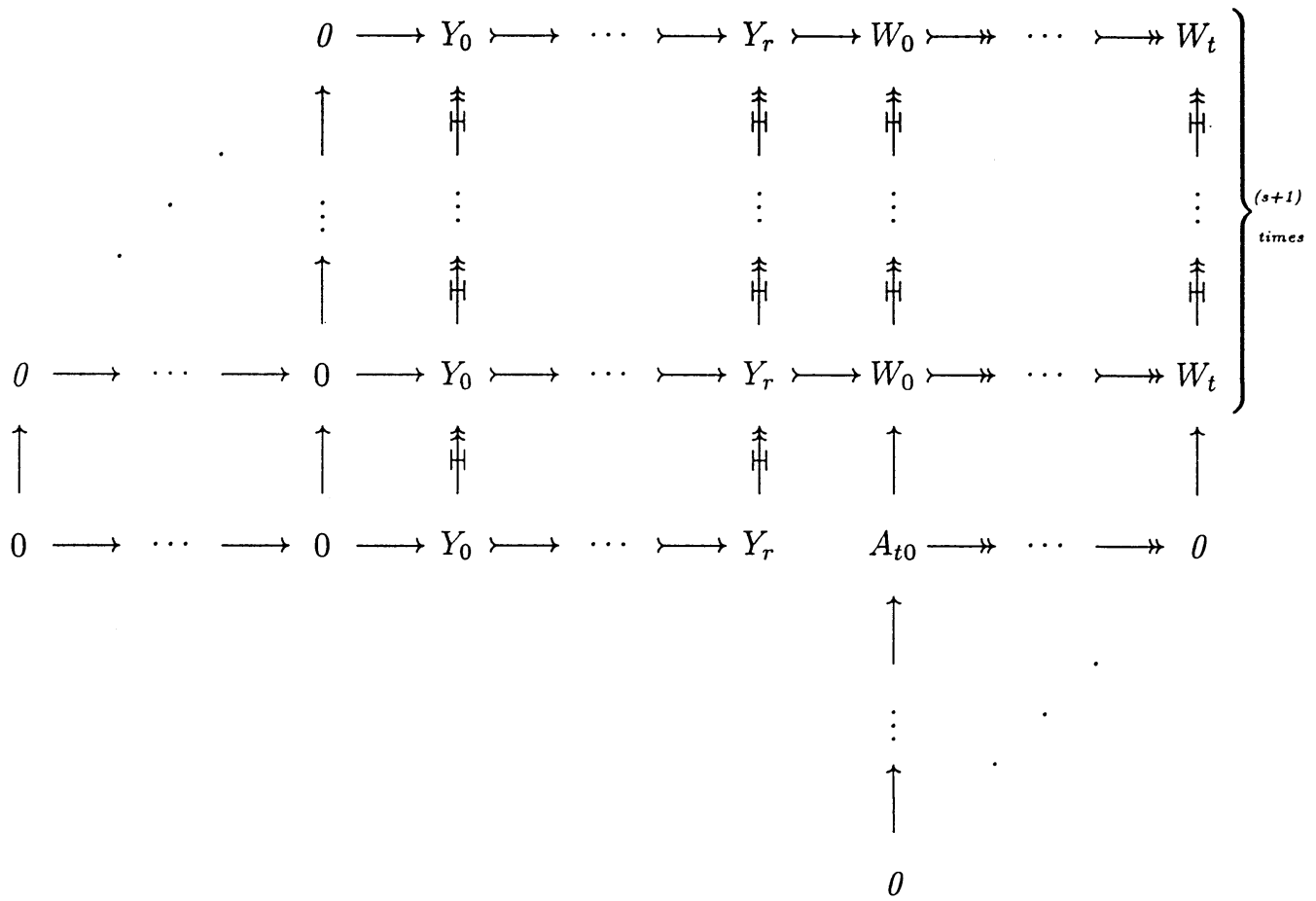

Proof. As before, it is enough to "freeze" one of the simplicial structures. We prove

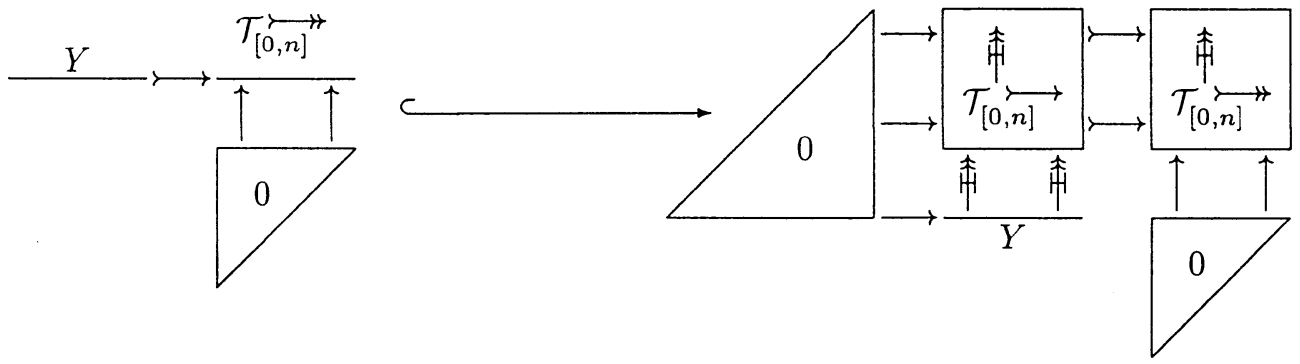

is a homotopy equivalence for every $Y$. The left hand side has the homotopy type of

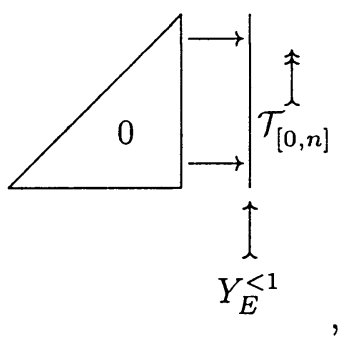

whereas the right hand side fibers over the contractible space 


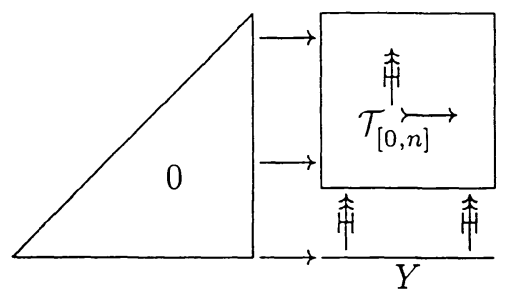

with the fiber

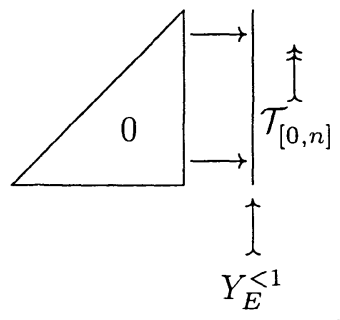

Hence the Lemma.

Lemma 3.8'=Lemma 3.8. The projection

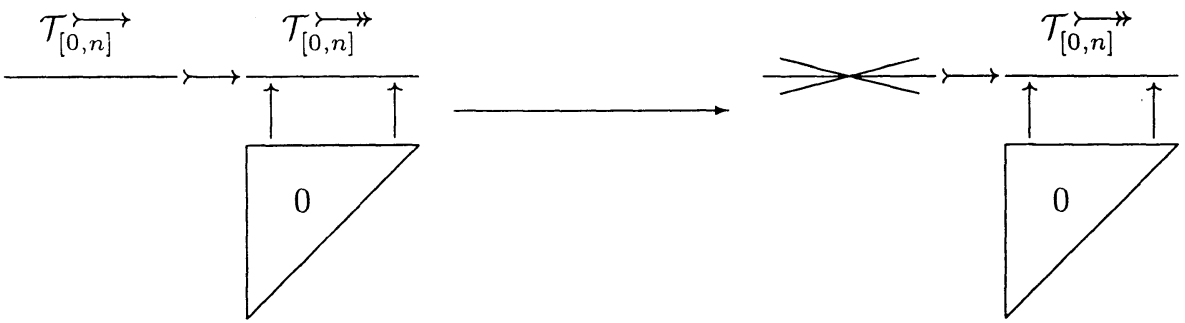

is a homotopy equivalence.

Proof. Being identical with Lemma 3.8, this trivial statement requires no new proof.

Conclusion. Combining everything we have done so far in this section, we deduce a commutative diagram whose columns are fibrations 

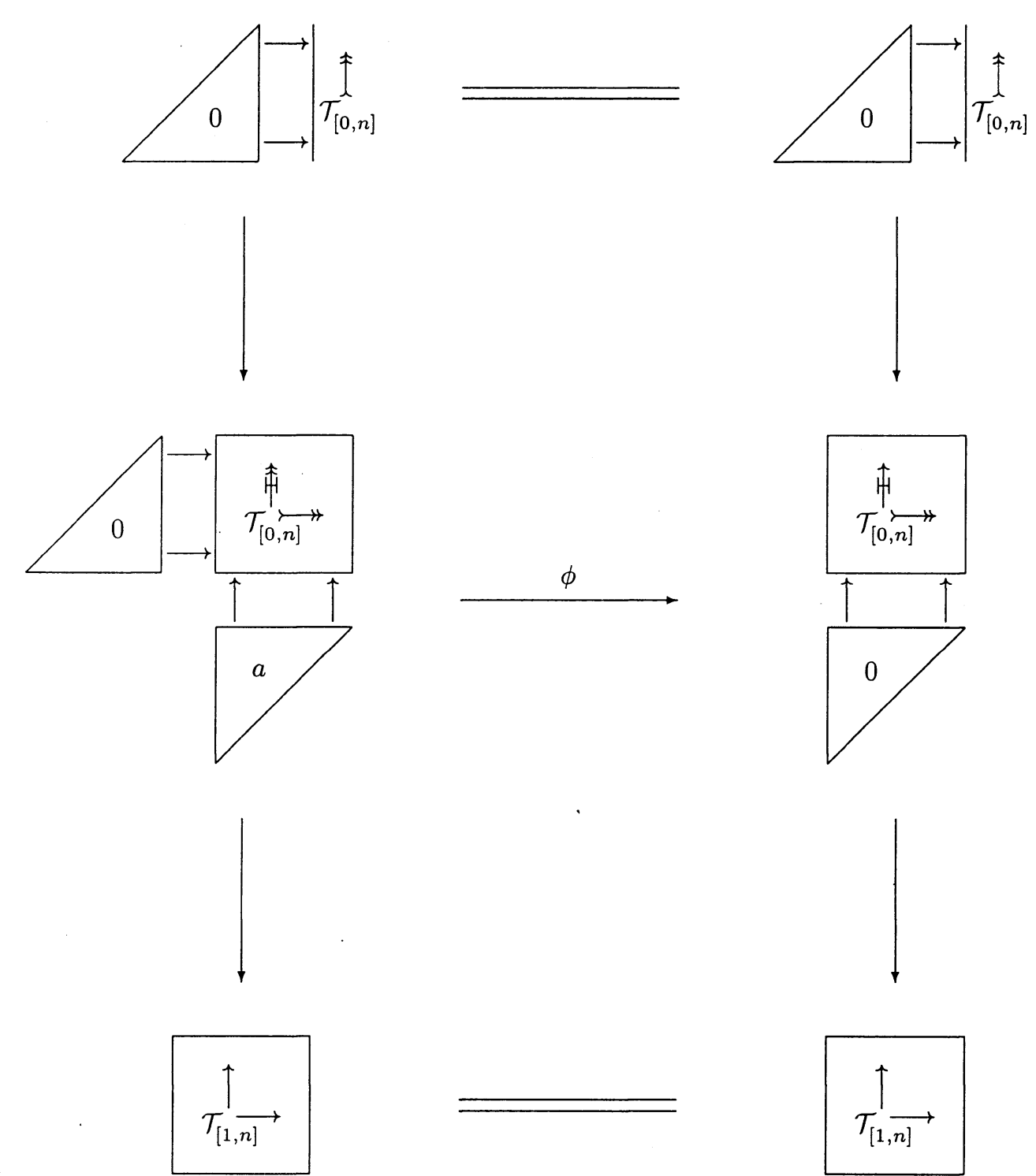

From the diagram we immediately deduce that the map $\phi$ is a homotopy equivalence. Command Center Summons for Another Gathering of the General Staff. Beyond this, our strategy will be to copy what was done in Section I.8. Precisely, we will now repeat the reasoning of Lemmas I.8.2-I.8.7. Because we will repeat them for both columns of our commutative diagram of fibrations just above, we will deduce two new models for the simplicial set

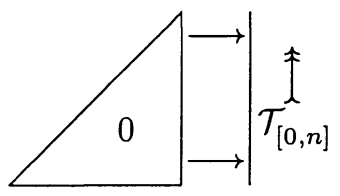

together with a map between them inducing the homotopy equivalence. The proof will then be complete when we show the map null homotopic.

General Staff Dismissed. 
Lemma I.8.2' The natural projection
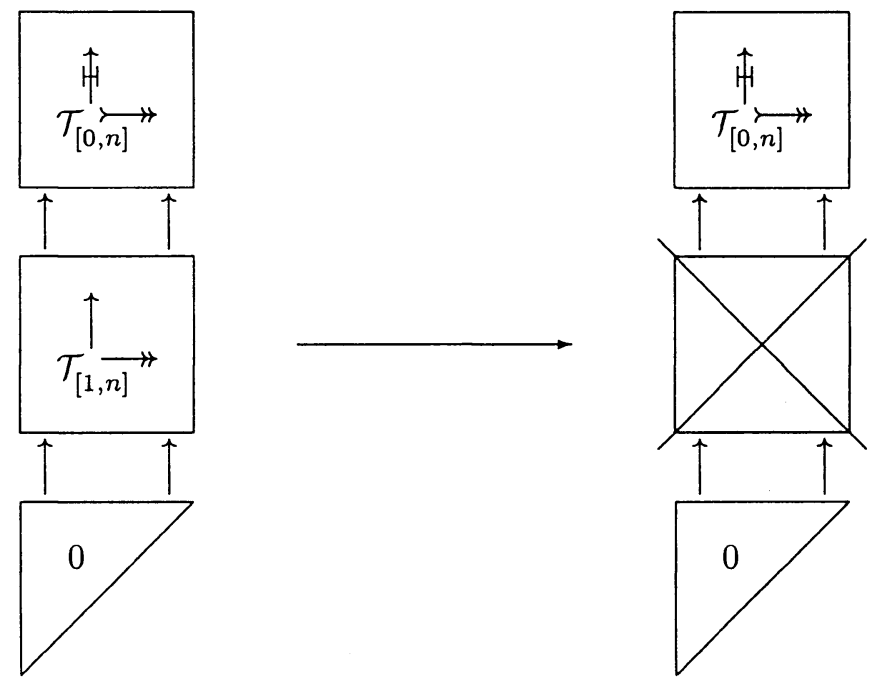

is a homotopy equivalence, as is the projection
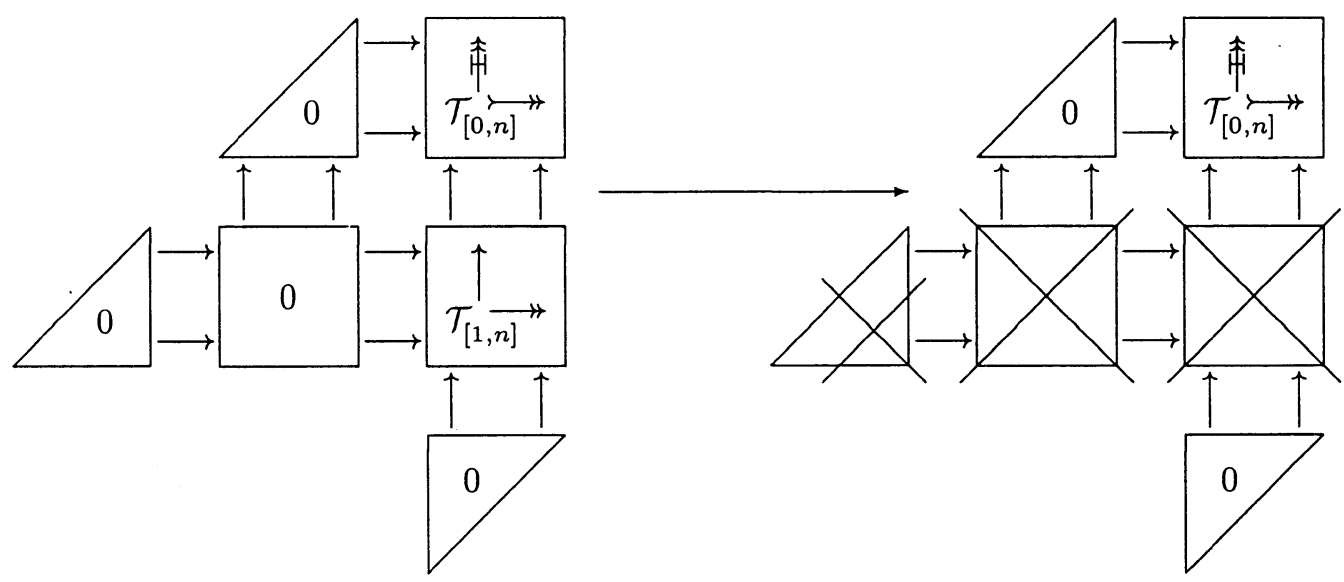

Proof. Because the case with kernels is somewhat more delicate, we will treat that case, leaving to the reader the proof without the kernels (which, by the way, is the same as the proof of Lemma I.8.2).

We need to establish the contractibility of the Segal fiber 


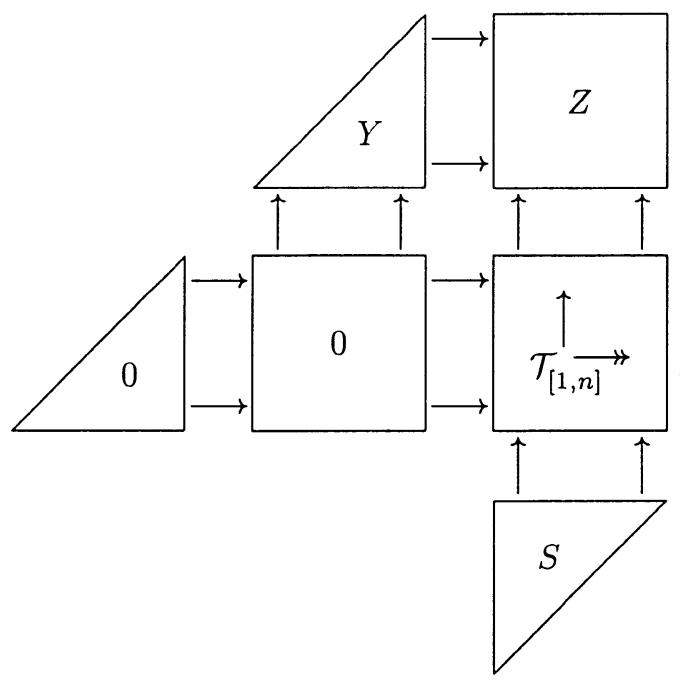

It is contacted by the homotopy

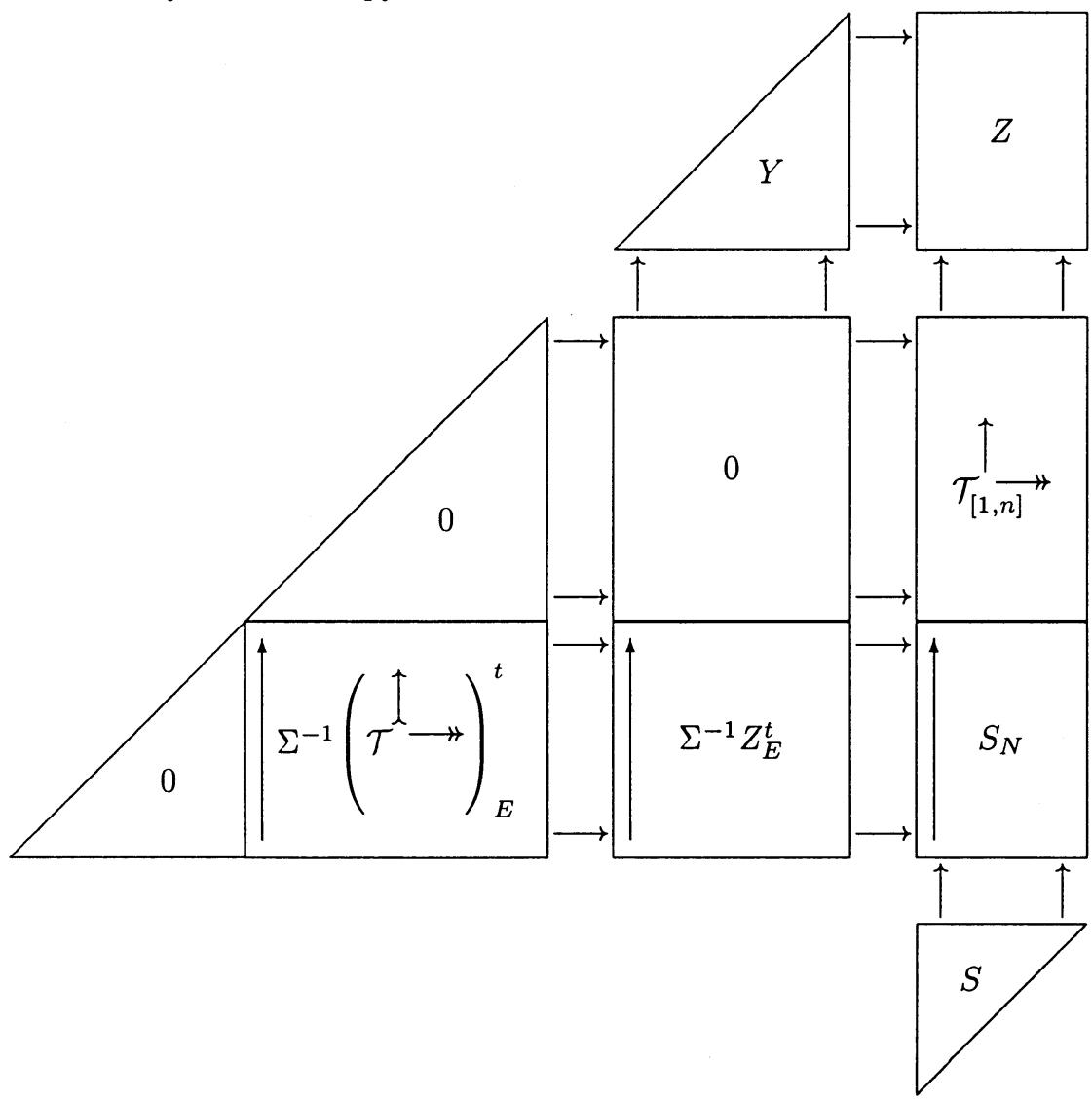

And the real point of the proof is that the kernels in the homotopy row of the diagram above are desuspensions of objects elsewhere in the diagram. This is because in a triangulated category, kernels and cokernels are the same, and this identification allows the simplex to reproduce. See also Remark I.5.5. This is therefore one argument that really fails without the coherent differentials. 
Lemma I.8.3': The natural map
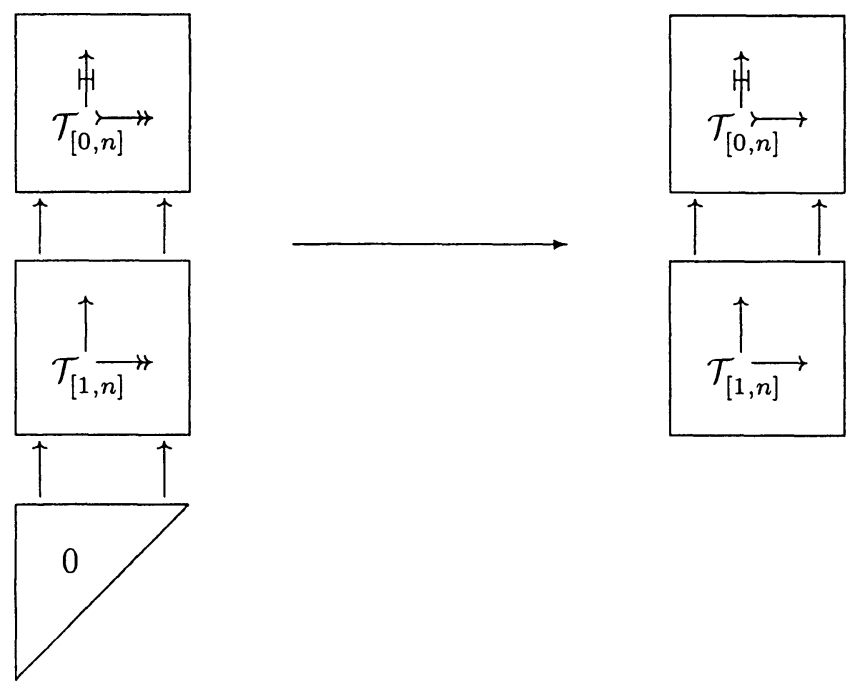

is a homotopy equivalence, as is the natural map

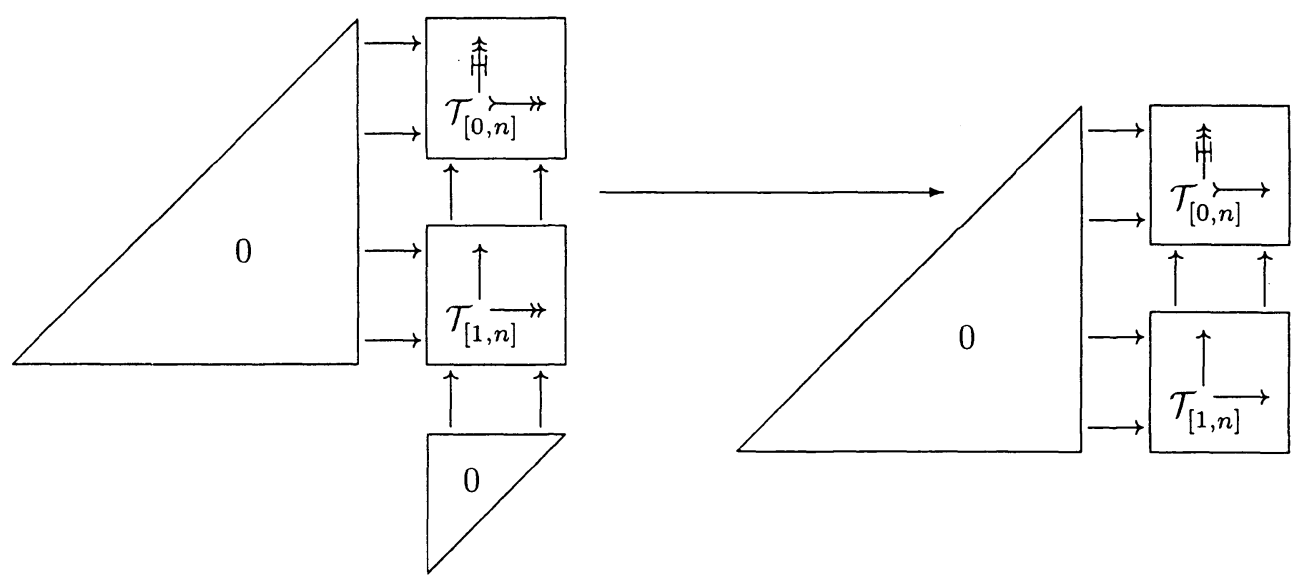

Proof. This time, the case with kernels and the case without are equally easy. Because in Section I.8 we treated the no-kernel case, we will do the other one here. Study the quarti-simplicial set and two projections 


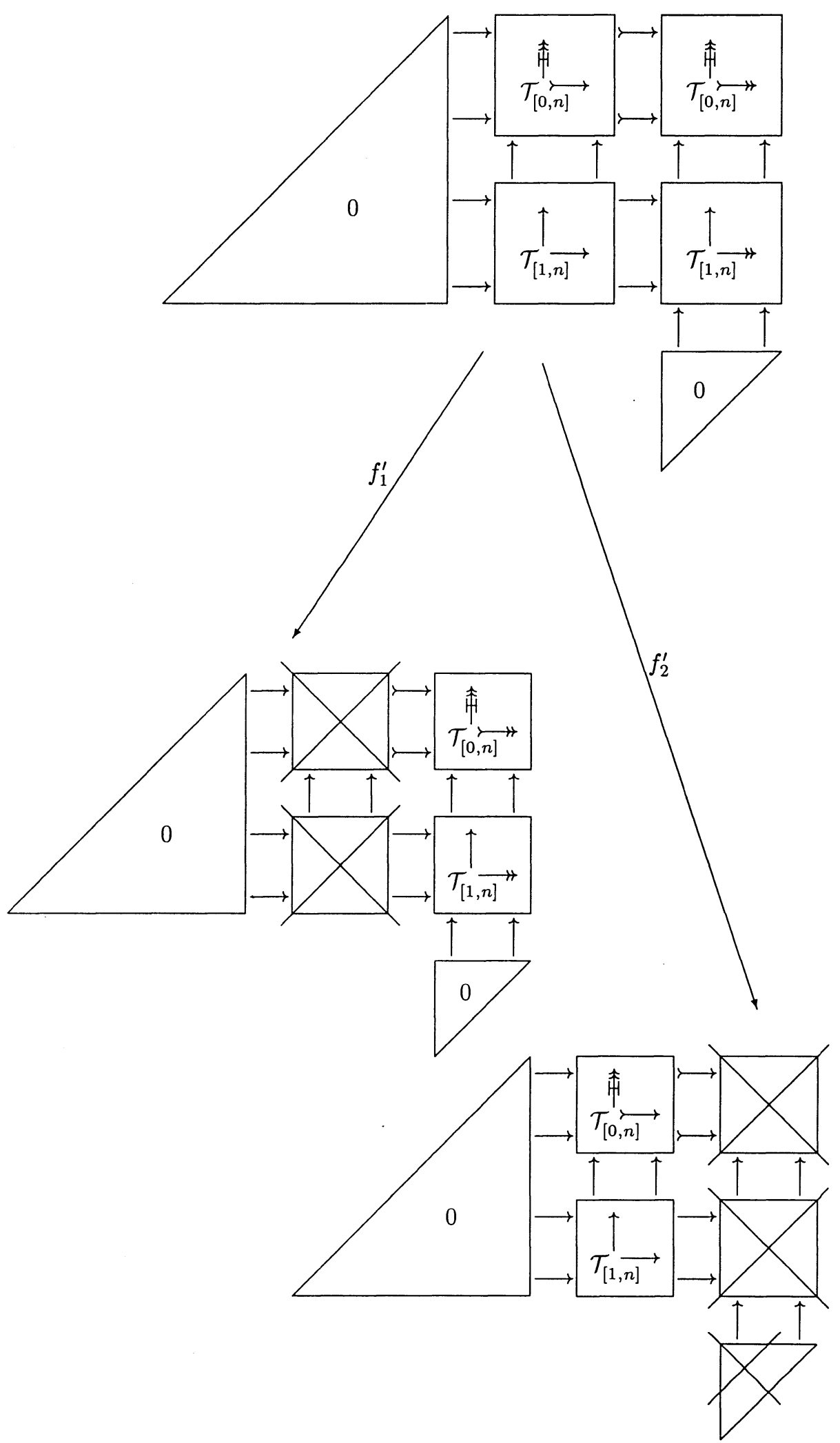


$f_{1}^{\prime}$ is a homotopy equivalence because on the fiber

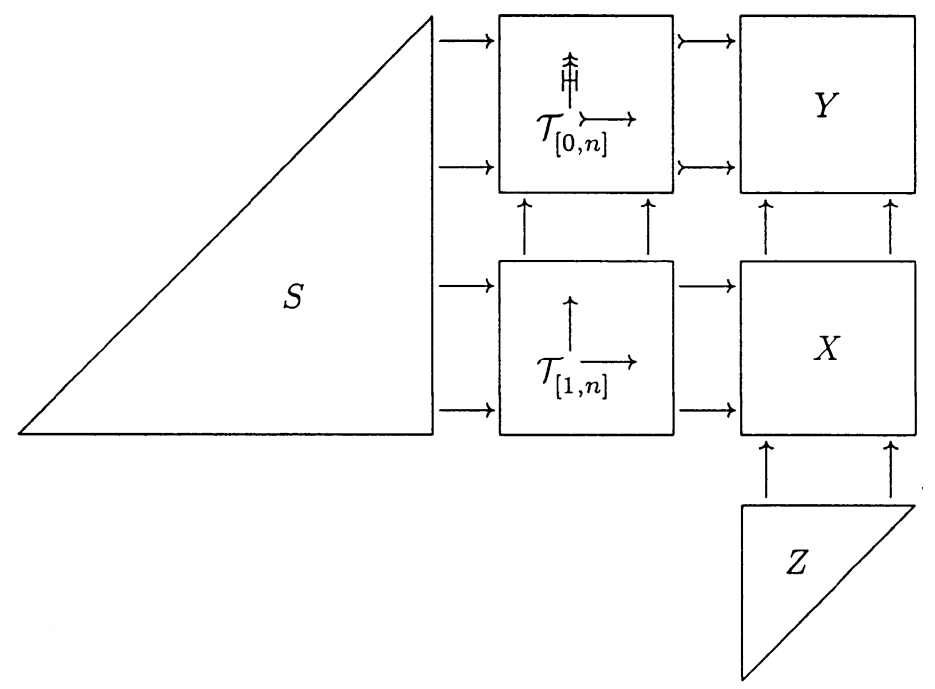

we can apply the contracting homotopy

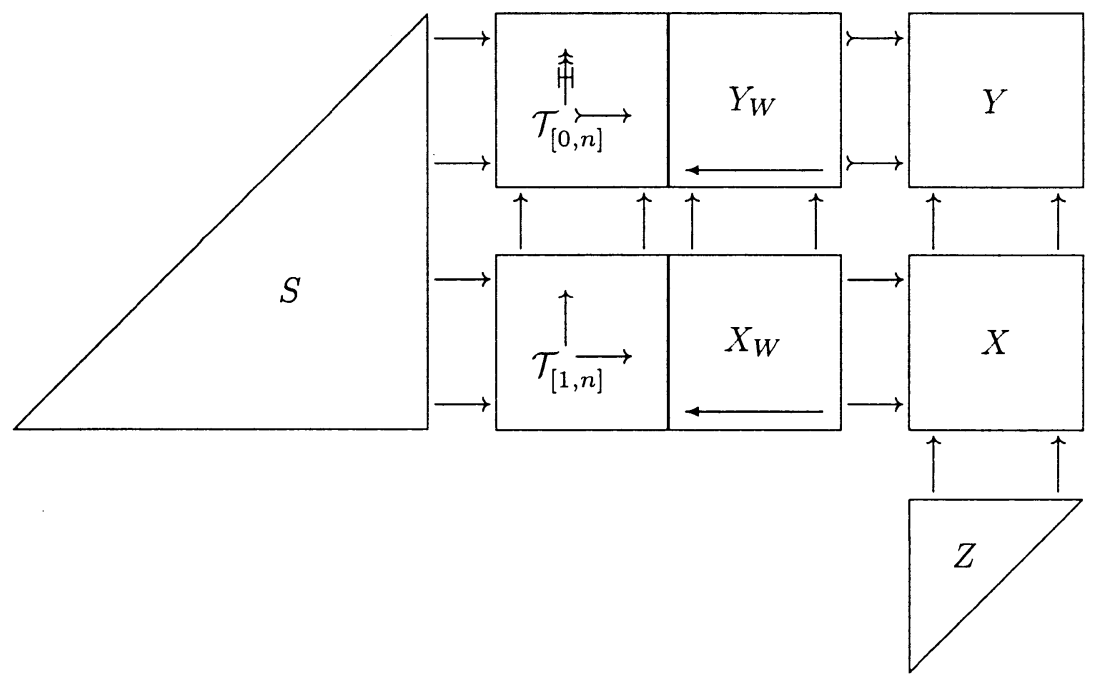

The map $f_{2}^{\prime}$ is slightly trickier. We resort to our favorite homotopy. The Segal fiber is the simplicial set 


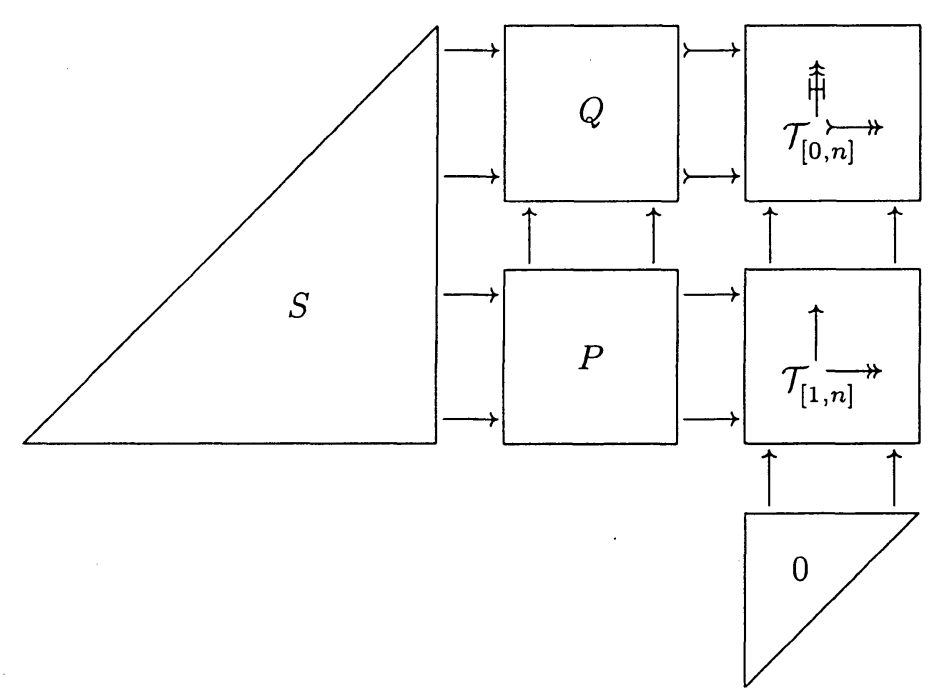

and we need to show it contractible. By the homotopy
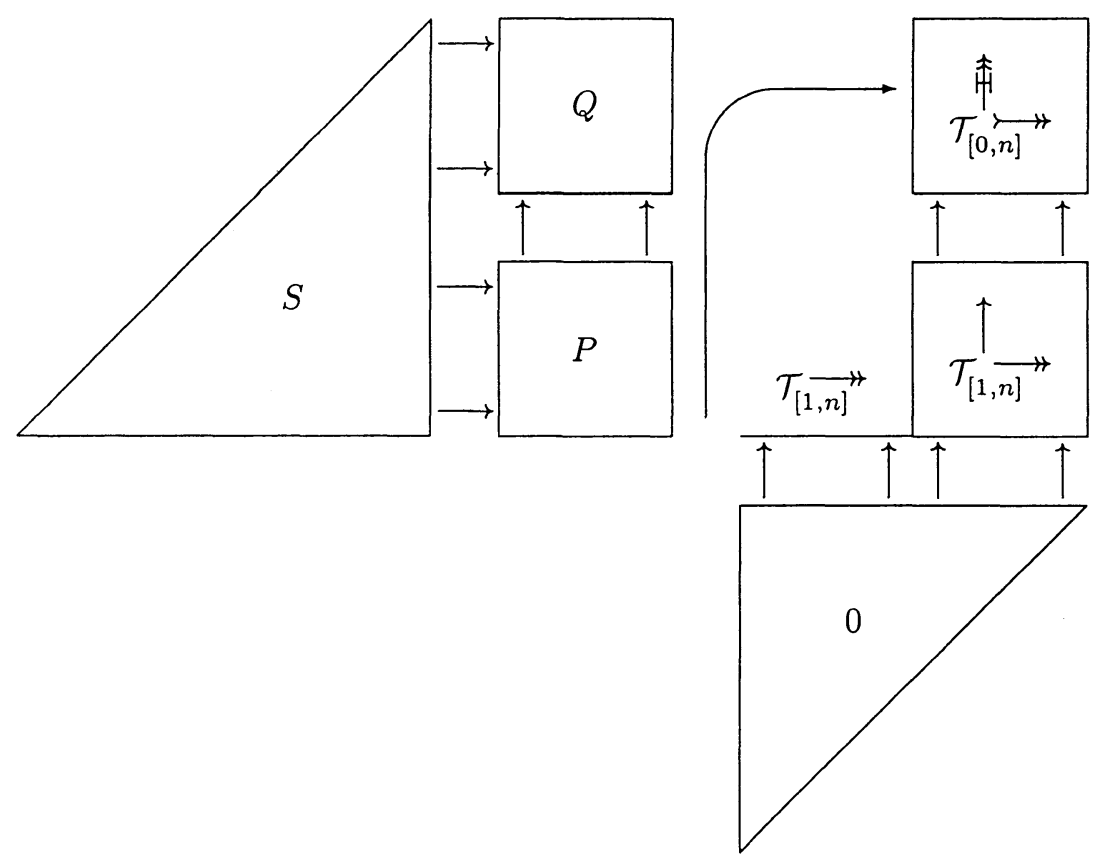

the identity on 


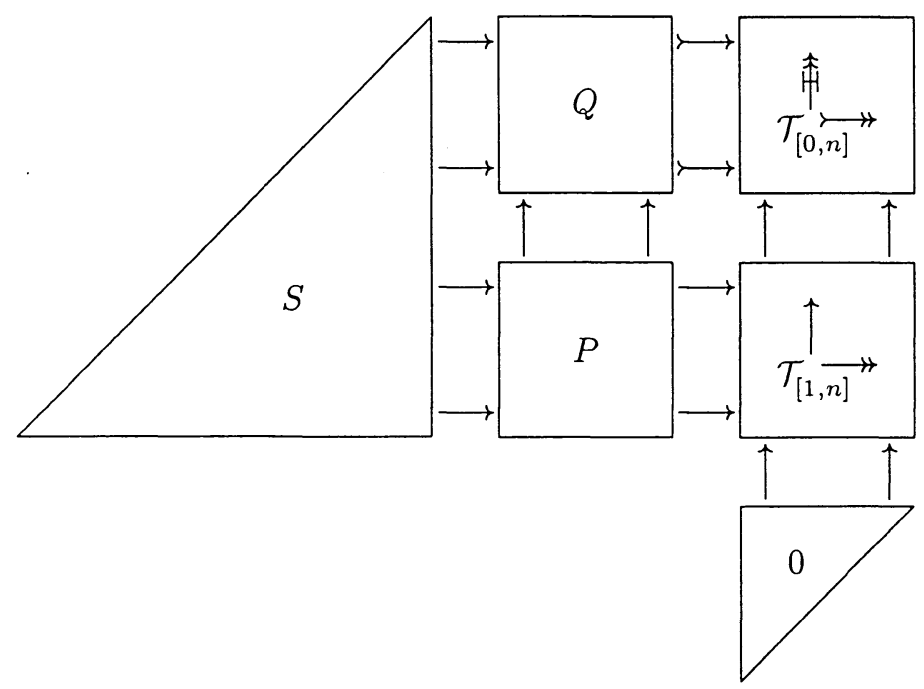

factors through the simplicial set

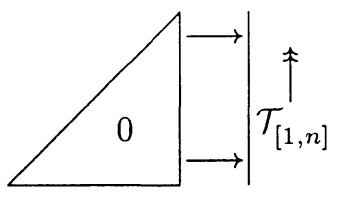

which is trivially contractible, by the contraction to the terminal object.

Lemma I.8.6'. The projection
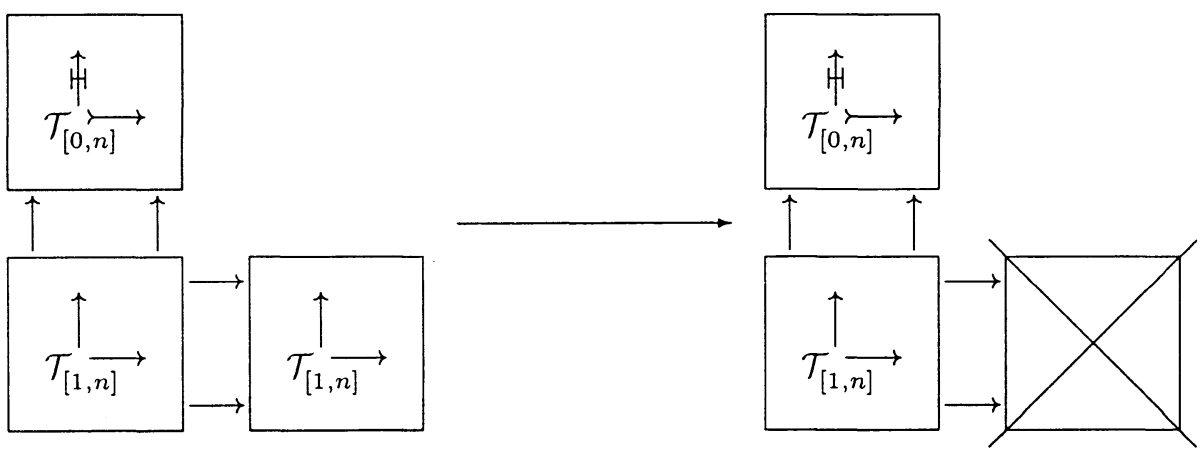

is a homotopy equivalence, as is the projection 

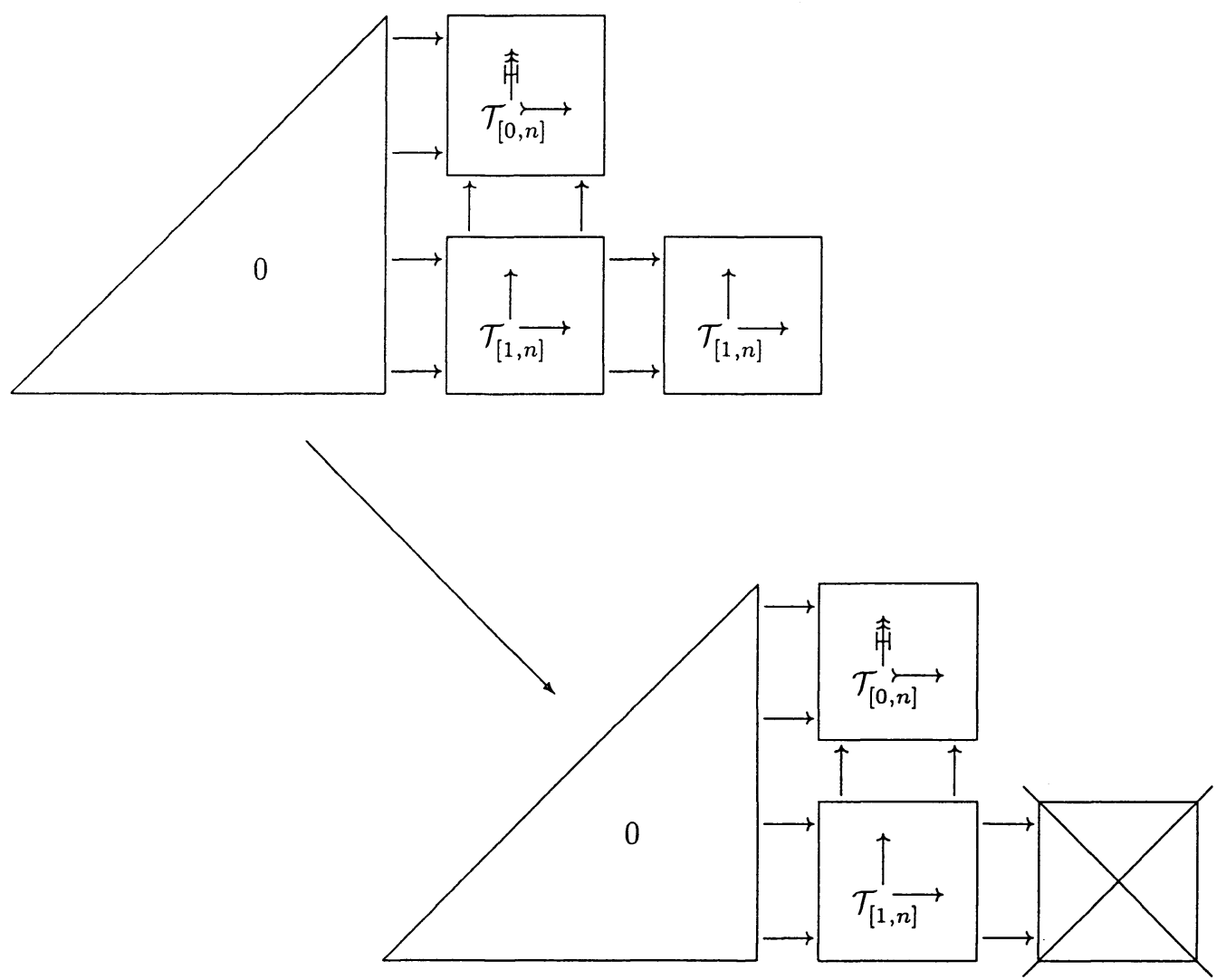

Proof. Once again, we treat the case with the kernels. The Segal fiber is the simplicial set

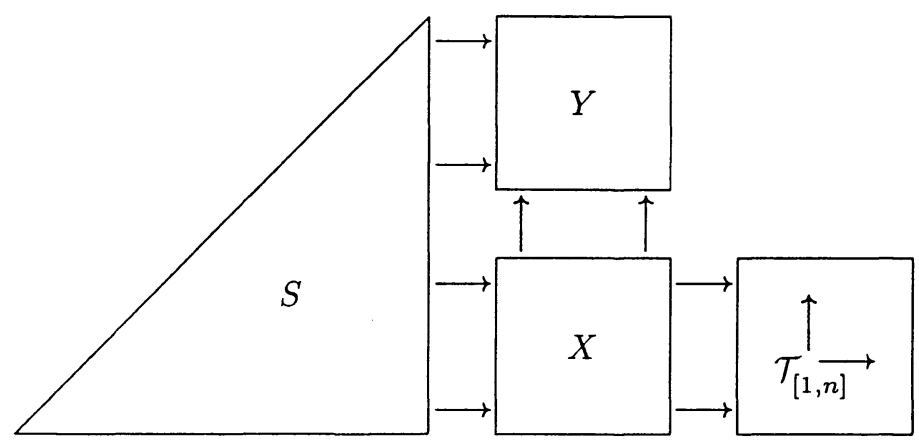

and it is contracted by the homotopy

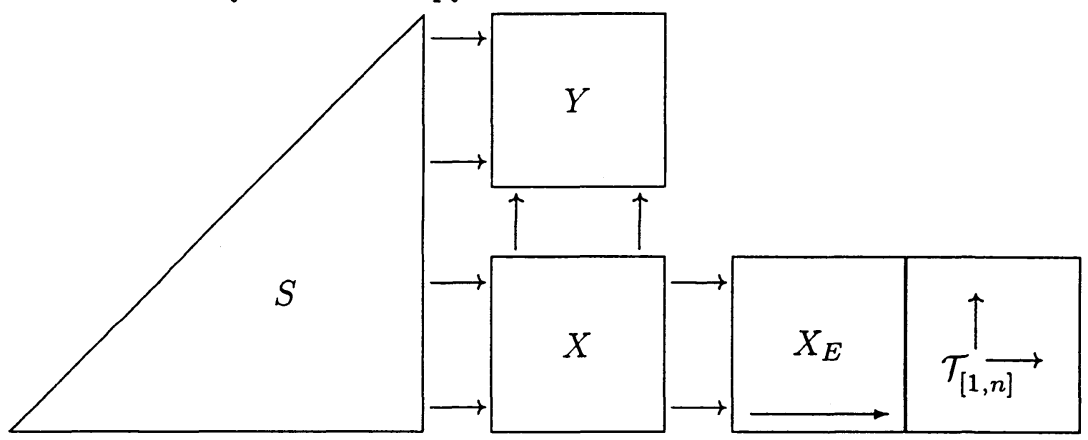


Lemma I.8.7'. The projection
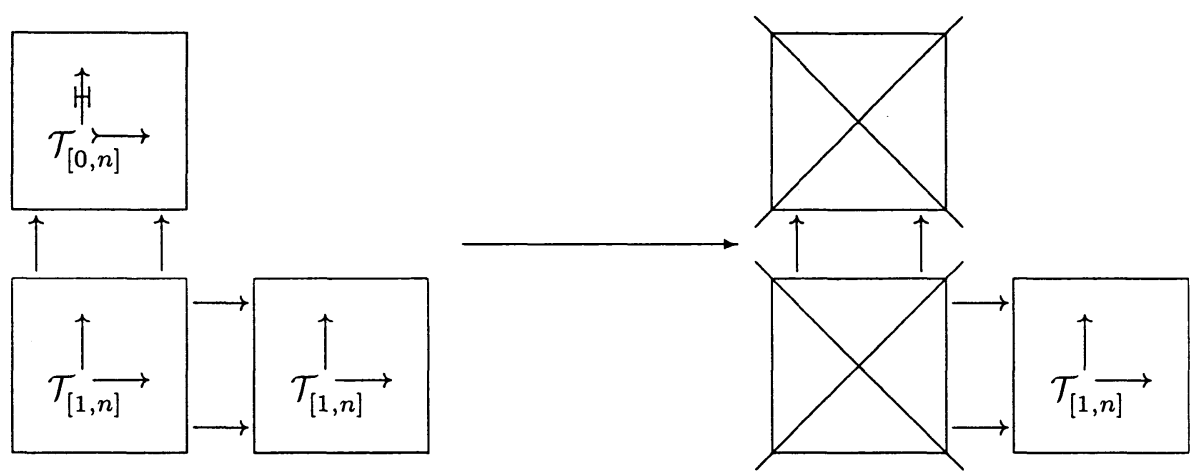

is a quasi-fibration, as is the projection
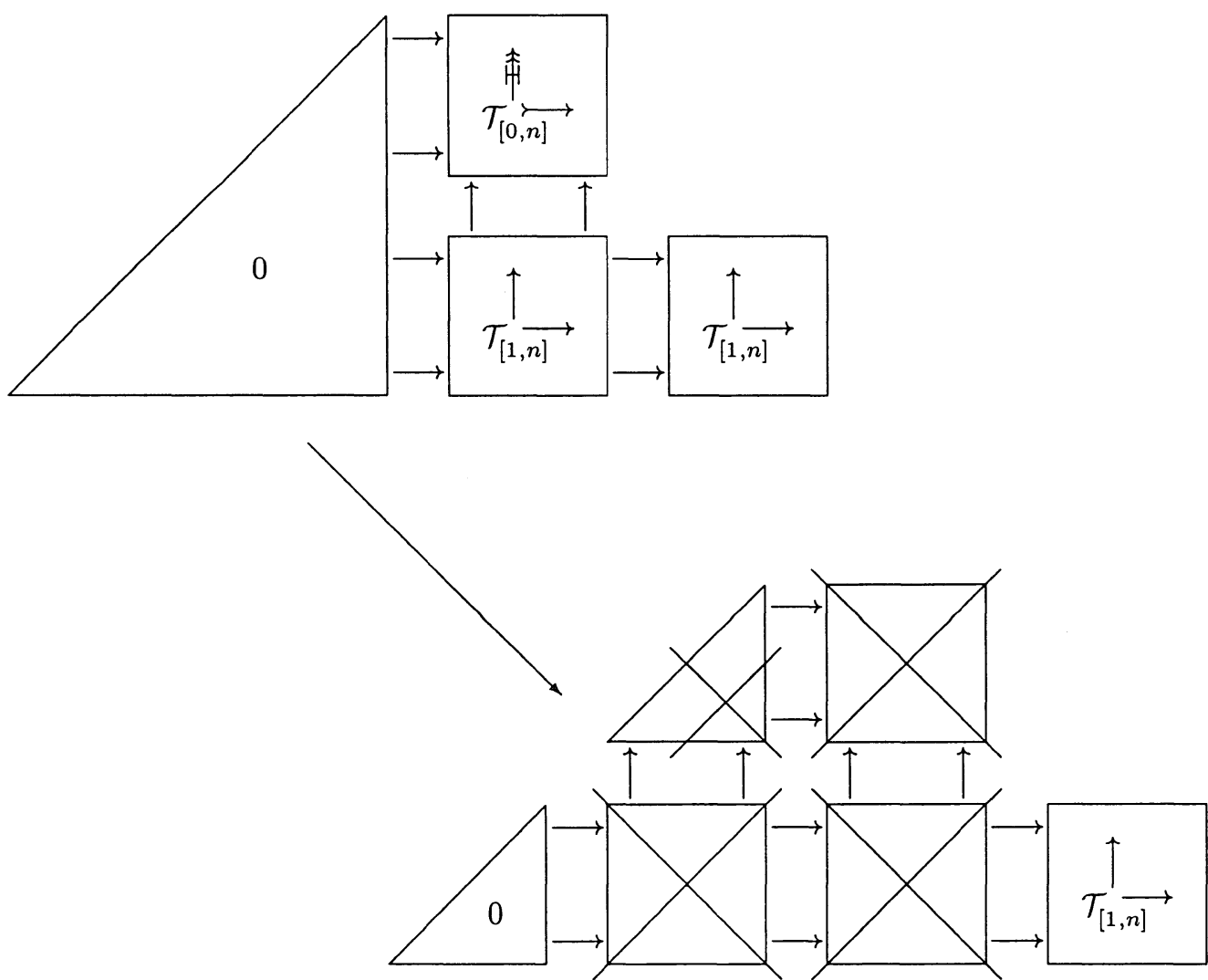

Proof. In the case with no kernels, this is Prototype Quasifibration 1.2, whereas in the case with differentials it is Prototype Quasifibration 1.4.

Conclusions So Far. There is a diagram whose columns are fibrations 

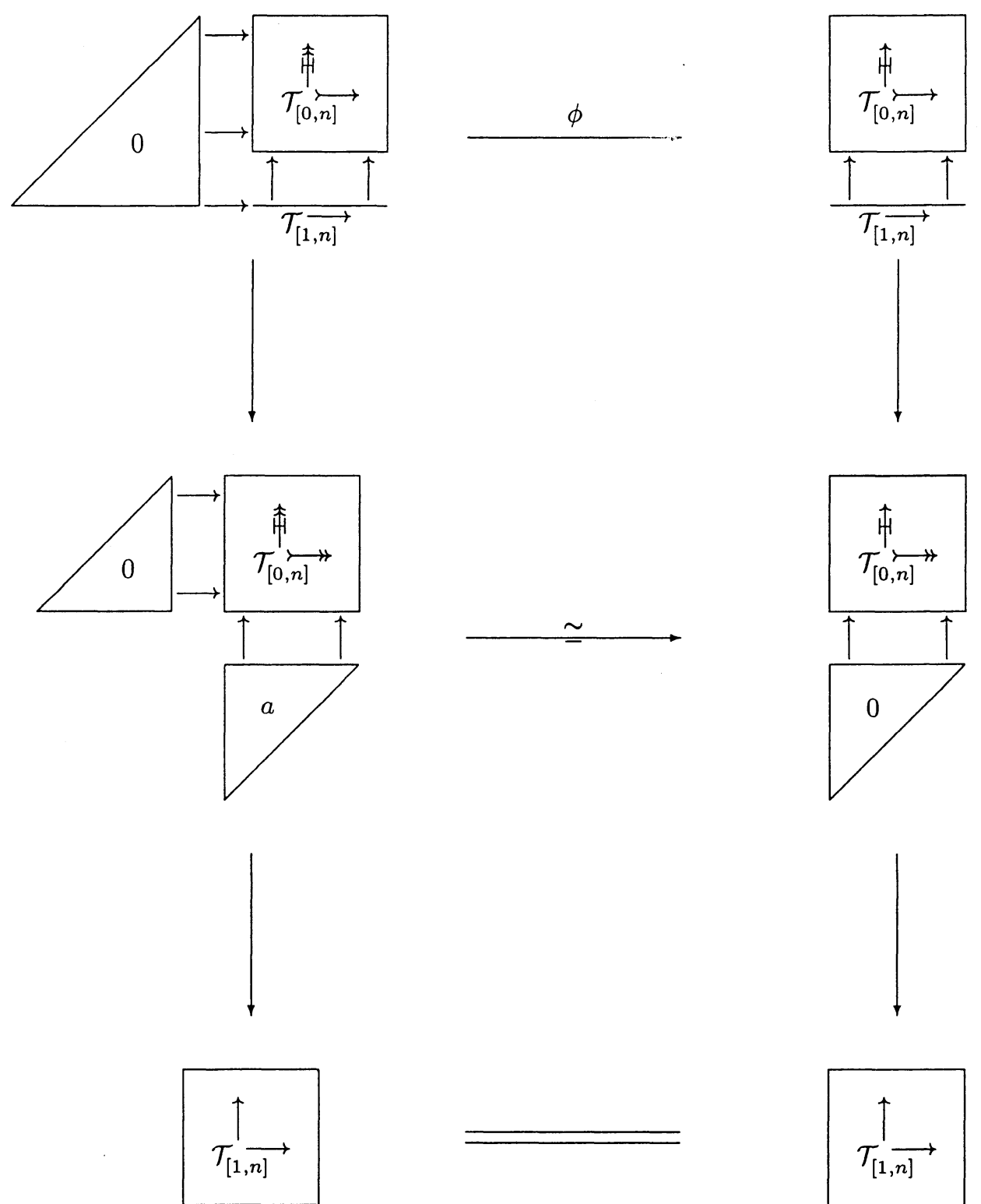

It immediately follows that the map $\phi$ is a homotopy equivalence. Because we already also know that the fiber in each column is homotopy equivalent to

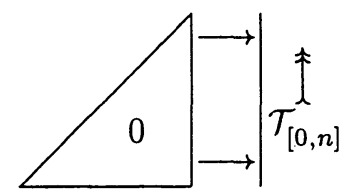

Theorem I.7.1 is an immediate consequence of

Lemma 3.9. The natural map 

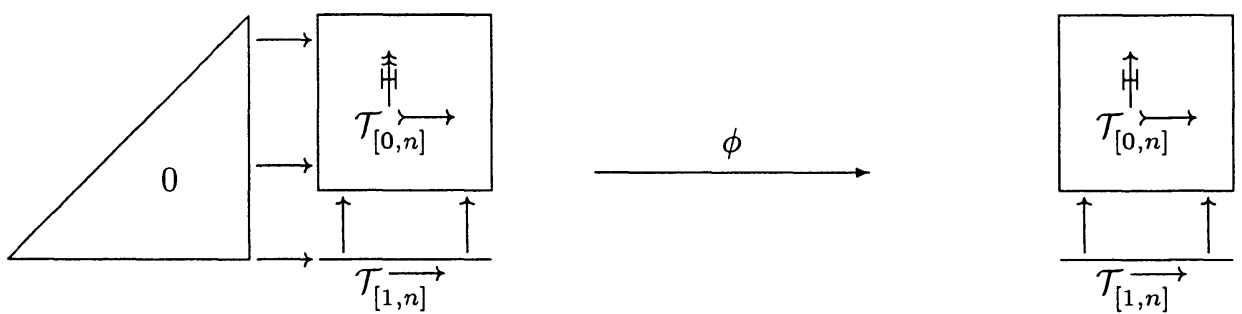

is null homotopic.

Proof. The point is that, up to homotopy, this map factors through

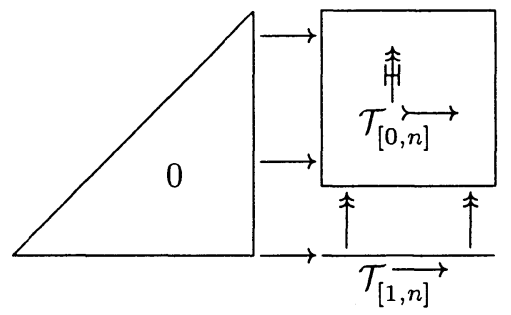

which is contractible. The map $\phi$ just forgets the kernels. Consider next the map
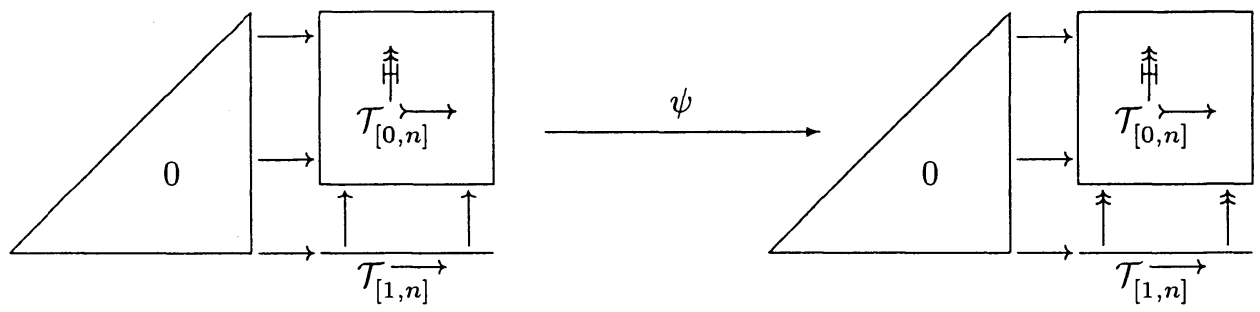

given by sending the simplex

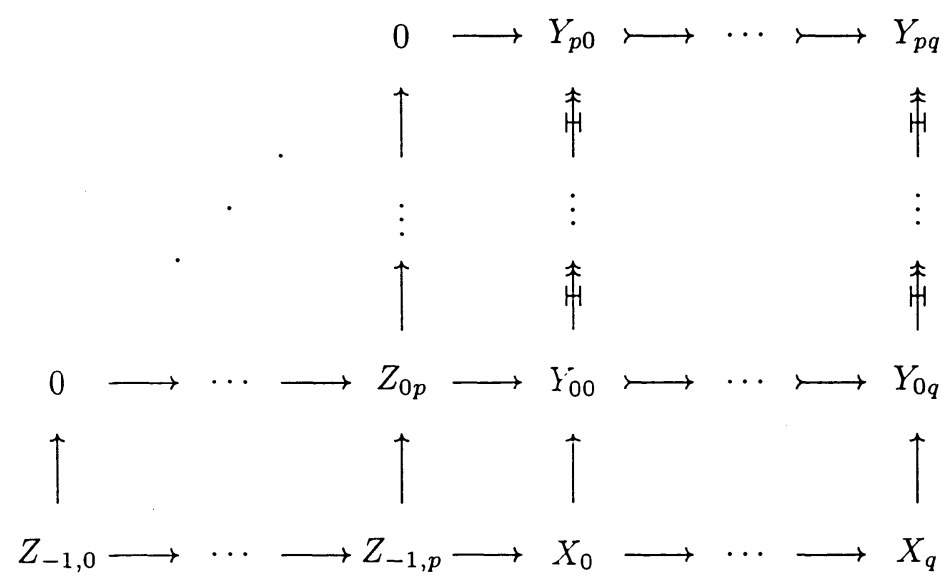

to the simplex 


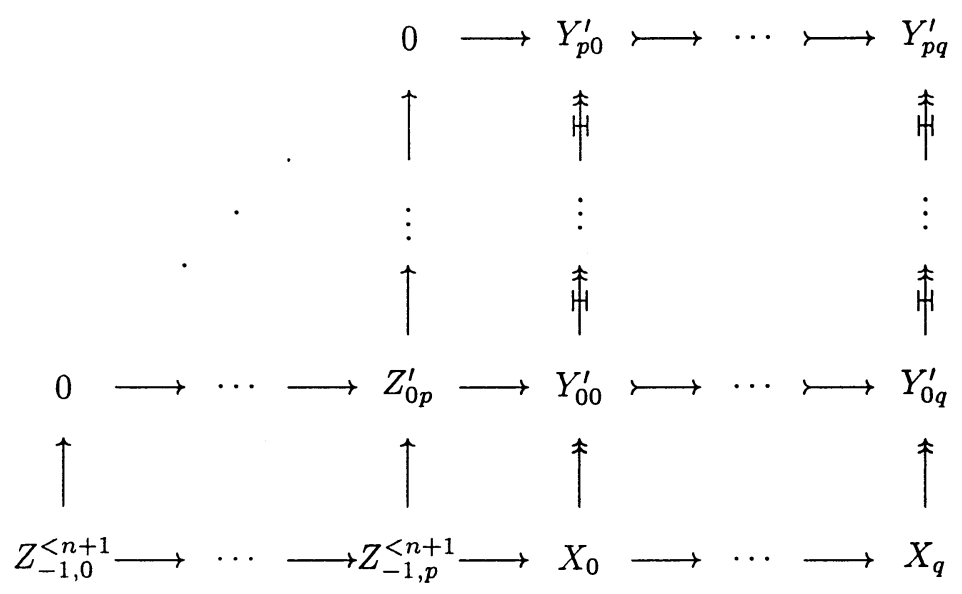

where $Z_{-1, j}^{<n+1}$ is the truncation of $Z_{-1, j}$ below degree $n$, and the other objects in the diagrams are the truncations forced by this. It is completely clear that the diagram
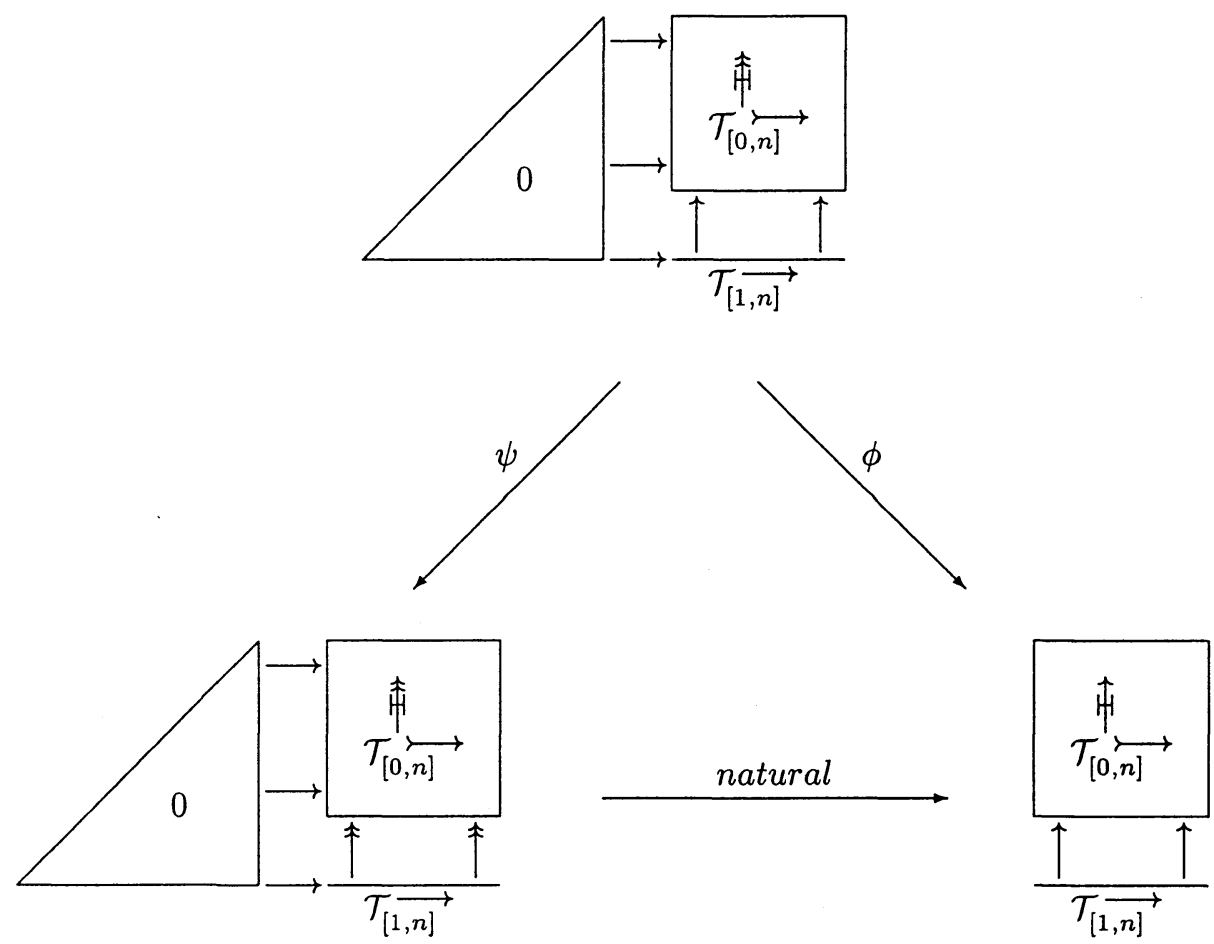

commutes up to homotopy.

If we want to write what would be our notation for the homotopy of $\phi$ to (natural) $\circ \psi$ in the diagram above, we note that $\phi$ is just the map . 

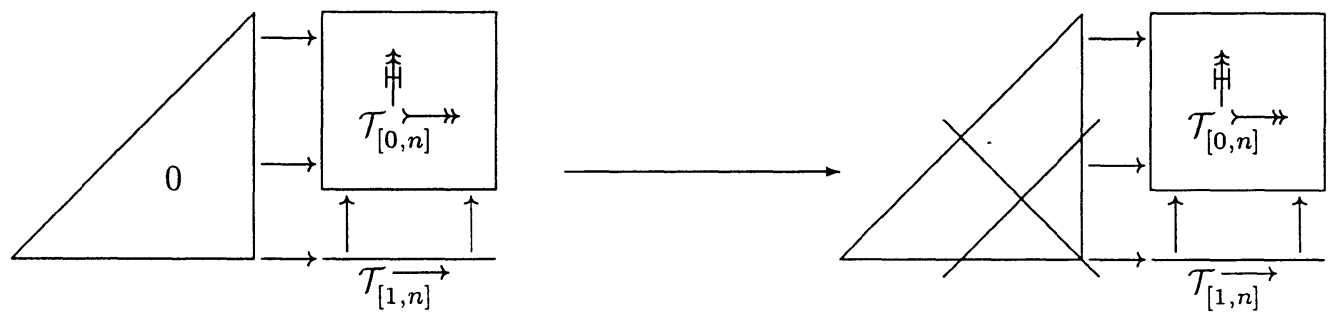

and the homotopy to $\psi \circ$ (natural) would be given by a picture looking something like

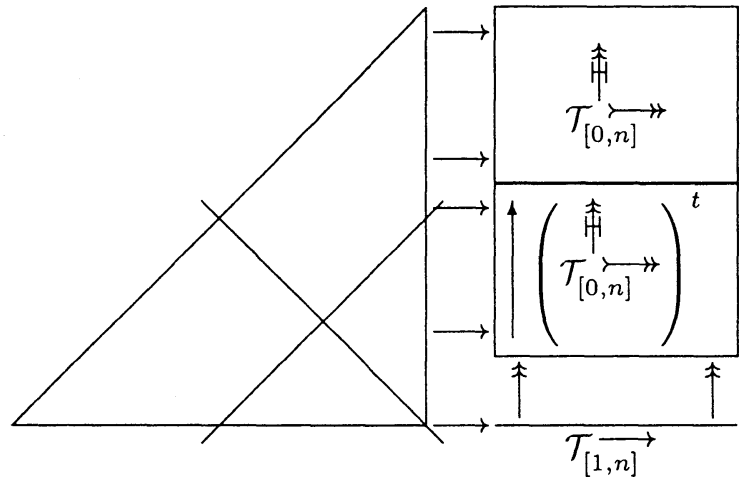

Since we are nearing the end of the article, the reader should feel free to improve on the author's notation; it could certainly use improvement.

This establishes our first assertion. Now we will show that

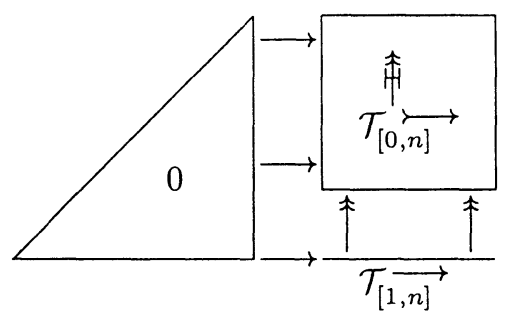

is contractible. Observe first that the inclusion

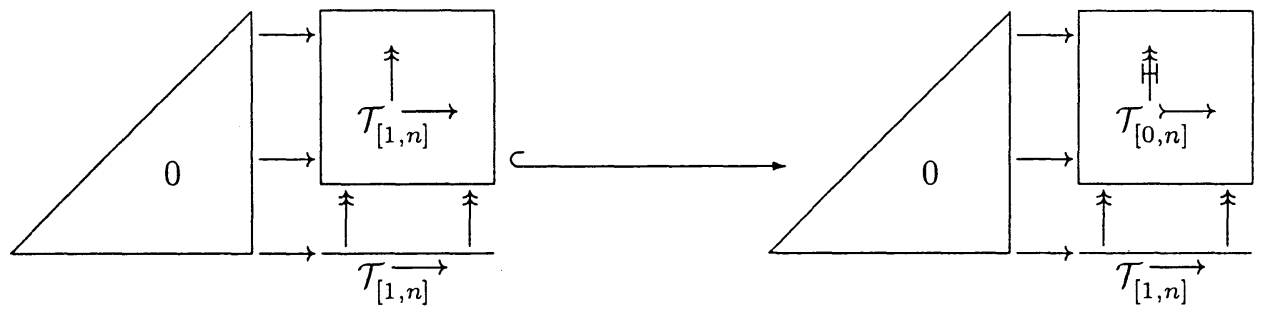

is a homotopy equivalence, by studying the trisimplicial set and the two projections 

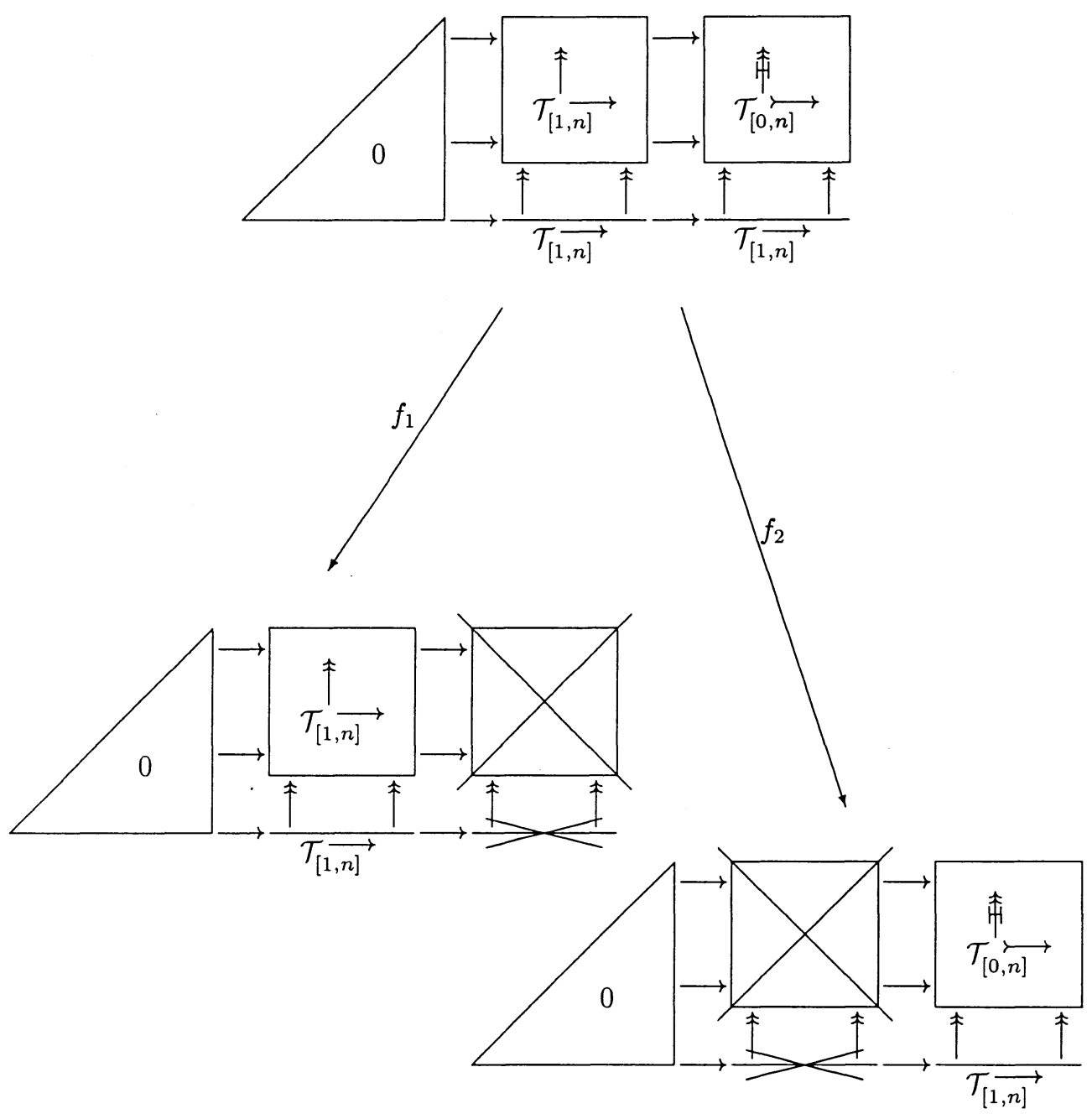

We want to show that each of $f_{1}$ and $f_{2}$ is a homotopy equivalence. The Segal fiber of $f_{1}$ is contracted by the homotopy

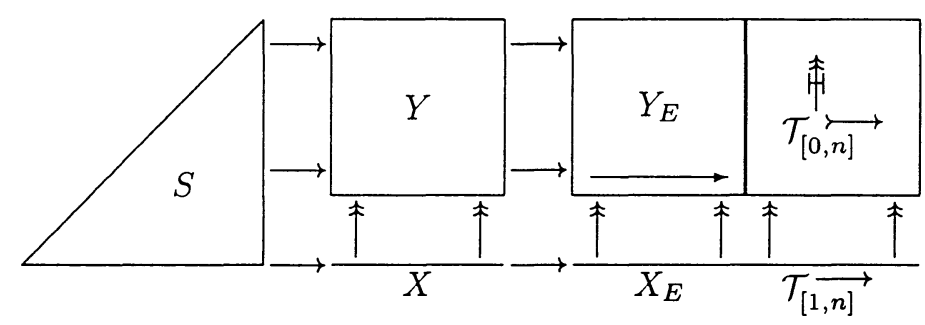

whereas the Segal fiber of $f_{2}$ is contracted by 


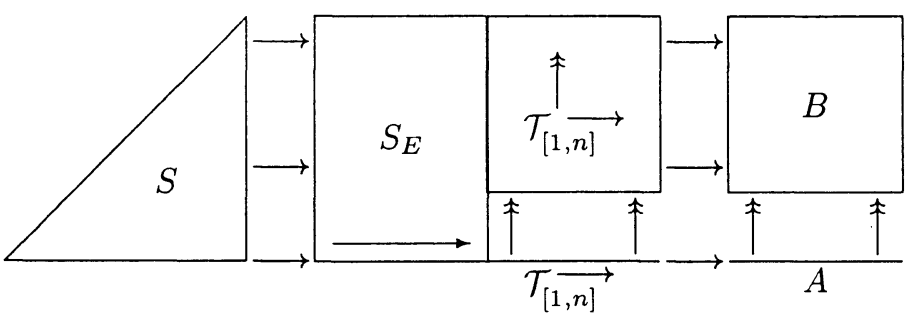

Finally, the simplicial set

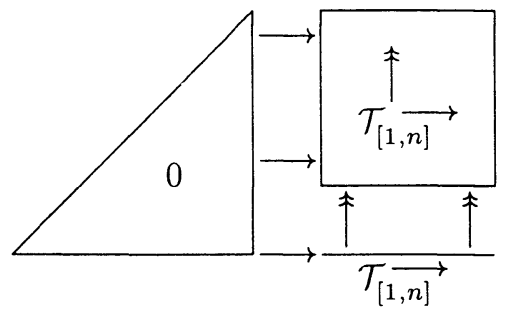

is clearly contractible.

This completes the proof, and the article. I will spare the reader the victory celebration by the general staff.

\section{REFERENCES}

[1] A. A. Beilinson, J. Bernstein and P. Deligne. Analyse et topologie sur les éspaces singuliers. Astérisque 100, Soc. Math. France (1982).

[2] A. Borel and J. P. Serre. Le théorème de RiemannRoch, Bull. Soc. Math. France 86 (1958), 97-136.

[3] C. Giffen, and A. Neeman. $K$-theory for triangulated categories. Preprint.

[4] R. Hartshorne. Residues and duality, SLN 20 (1966).

[5] V. A. Hinich AND V. V. SChechtman. Geometry of a category of complexes and algebraic $K$-theory. Duke Math. J. 52 (1985), 399-430.

[6] A. NeEman. Some new axioms for triangulated categories. J. of Algebra 139 (1992) 221-255.

[7] A. NeEman. The Brown Representability Theorem and phantomless triangulated categories. J. of Algebra 151 (1992)'118-155.

[8] D. Quillen. Higher algebraic $K$-theory I, SLN 341 (1973), 85-147.

[9] R. Thomason and T. Trobaugh. Higher algebraic K-theory of schemes and of derived categories. In: The Grothendieck Festschrift ( a collection of papers to honor Grothendieck's 60'th birthday) Volume 3 pp. 247-435, Birkhäuser 1990.

[10] J. L. Verdier. Catégories dérivées, état 0. SGA $4 \frac{1}{2}, 262-308$ (SLN 569, 1977).

[11] F. Waldhausen. Algebraic $K$-theory of generalized free products I, II. Ann. of Math. 108 (1978), 135-256.

[12] F. WALDhauSEN. Algebraic K-theory of spaces. SLN 1126 (1985), 318-419. 
